\title{
Characterization of the Immune Response to Lipopolysaccharide in Early Pregnant Ewes as a Model to Study Bacterial Infection Induced Embryonic Loss
}

Jessalyn Marie Hadfield

Follow this and additional works at: https://researchrepository.wvu.edu/etd

\section{Recommended Citation}

Hadfield, Jessalyn Marie, "Characterization of the Immune Response to Lipopolysaccharide in Early Pregnant Ewes as a Model to Study Bacterial Infection Induced Embryonic Loss" (2017). Graduate Theses, Dissertations, and Problem Reports. 5731.

https://researchrepository.wvu.edu/etd/5731

This Dissertation is protected by copyright and/or related rights. It has been brought to you by the The Research Repository @ WVU with permission from the rights-holder(s). You are free to use this Dissertation in any way that is permitted by the copyright and related rights legislation that applies to your use. For other uses you must obtain permission from the rights-holder(s) directly, unless additional rights are indicated by a Creative Commons license in the record and/ or on the work itself. This Dissertation has been accepted for inclusion in WVU Graduate Theses, Dissertations, and Problem Reports collection by an authorized administrator of The Research Repository @ WVU.

For more information, please contact researchrepository@mail.wvu.edu. 
Characterization of the Immune Response to Lipopolysaccharide in Early Pregnant Ewes as a Model to Study Bacterial Infection Induced Embryonic Loss

Jessalyn Marie Hadfield

Dissertation submitted to the Davis College of Agriculture, Natural Resources and Design at West Virginia University in partial fulfillment of the requirements for the degree of

Doctor of Philosophy in Reproductive Physiology

\author{
Robert A. Dailey, Ph.D., Chair \\ Scott A. Bowdridge, Ph.D. \\ Eugene E. Felton, Ph.D. \\ Ida Holásková, Ph.D. \\ Jianbo Yao, Ph.D.
}

Division of Animal and Nutritional Sciences

Morgantown, West Virginia

2017

Keywords: Early Embryonic Loss, Lipopolysaccharide, Inflammation, Omega-3

Polyunsaturated Fatty Acids, Breed Differences, Sheep,

Copyright 2017 Jessalyn Marie Hadfield 


\begin{abstract}
Characterization of the immune response to lipopolysaccharide in early pregnant ewes as a model to study bacterial infection induced embryonic loss.

\section{Jessalyn Marie Hadfield}

An immunological balance has to be established during pregnancy that protects the mother yet tolerates the semi-allogenic fetus. To understand the innate immune response during bacterial infections that may cause early embryonic loss, a lipopolysaccharide (LPS) treated sheep model was used. Two objectives of this study were to examine if omega-3 PUFAs in the form of supplementary whole flaxseed could reduce the inflammatory response to an LPS challenge and to examine if there is a differential immune response to LPS in Suffolk and Dorset ewes. A total of 3 experiments were conducted; two investigating the effect of supplement and one investigating the effect of breed. Estrus was synchronized by CIDR insertion for 5 days, followed by $20 \mathrm{mg}$ of $\mathrm{PGF}_{2 \alpha}$ at CIDR withdrawal. Early pregnant ewes received via the jugular vein, either phosphate buffered saline (PBS) (3 ml) or LPS ( $2.5 \mu \mathrm{g} / \mathrm{kg}$ ). Blood was collected via jugular venipuncture at hour: $0,0.25,0.5,0.75,1,1.5,2,3,4,5,6,9,12$, and 24 . Whole blood samples were used to determine white blood cell counts (WBCs) before centrifugation to collect white blood cells for RNA extraction. Rectal temperature and change in behavior/physical (lethargy, coughing, nasal discharge, absence of eating) appearance were recorded hourly. Real-time PCR was performed for expression of cytokines (CXCL8, IL6, TNFa, IFN $\gamma$, IL-10, and TGF $\beta$ ), receptors (TLR4, MRC1), enzymes (COX2, SOD2), transcription factors (NF-kB, PPAR $\gamma$, and Foxp3) and complement component 3. In all experiments, temperature increased in response to LPS, peaking at hour 4 before returning to normal by hours 6-9; WBCs dropped by hour 1 before returning to normal by hours 6-9. In trial 1 of the supplement study (flaxseed versus a control supplement) (Dorset ewes flaxseed + LPS n=3; flaxseed + PBS n=3; control + LPS n=5; control + PBS n=5), LPS increased haptoglobin and cortisol levels and affected gene transcription of CXCL8, IFN $\gamma$, TLR4, MRC1, SOD2, Foxp3 (by hour), and C3.There was a diet effect with regard to cortisol and gene expression of CXCL8, and TLR4. There was a diet $\mathrm{x}$ LPS interaction with regard to temperature, WBCs (by hour), haptoglobin, serum amyloid A and gene expression of CXCL8, IL-6, and TLR4. In trial 2 of the supplement study (Dorset ewes flaxseed + LPS n=11, flaxseed + PBS $\mathrm{n}=10$, control + LPS $\mathrm{n}=11$, control + PBS $n=10$ ), LPS increased haptoglobin and cortisol and affected gene expression of CXCL8, TLR4, MRC1, SOD2, PPAR $\gamma$, Foxp3, and C3. There was a diet effect on cortisol and gene expression of C3.There was a diet $x$ LPS interaction with regard to temperature and cortisol. In the breed effects study (Dorset + LPS n=11; Dorset + PBS n=10; Suffolk + LPS n=16; Suffolk + PBS n=16), LPS increased cortisol and affected gene transcription of CXCL8, TLR4, MRC1, SOD2, PPAR $\gamma$, and C3. There was an effect of breed on temperature; haptoglobin, serum amyloid A (by hour), and cortisol levels; and gene transcription of IL-6, IFN $\gamma$, IL10, TLR4, COX2, SOD2, PPAR $\gamma$, Foxp3, and C3. There was a breed x LPS interaction on change in temperature from hour 0 , the frequency of behavior/physical changes; haptoglobin, serum amyloid A (by hour), and cortisol; and gene transcription of IL-6 and C3. Pregnancy status was assessed at $25 \mathrm{dpc}$ with transrectal ultrasound and progesterone was measured in plasma samples. In trial 1 of the supplement study, flaxseed increased progesterone but it did not differ between groups in trial 2 . There were no differences in progesterone between the breeds tested. The number of ewes that lambed in each treatment group was not different in any of the experiments. In summary, acute infections may cause embryonic loss by shifting the environment to be pro-inflammatory. There was no clear benefit of supplementary $\omega-3$ PUFAs in reducing the inflammatory response. Suffolk ewes had an elevated inflammatory response to LPS compared to Dorset ewes and may be more susceptible to embryonic loss in response to infection. 


\section{Acknowledgments}

I would like to thank my advisor, Dr. Robert Dailey for allowing me to pursue this degree and to my other committee members Dr. Scott Bowdridge, Dr. Felton, Dr. Holásková, and Dr. Yao for their time, experience, knowledge, and encouragement along the way. I value everything I have learned from them, both in and out of a formal classroom setting. I would also like to thank Dr. Inskeep for always being interested in how students are doing and more than willing to help with anything they may need. I am grateful for your suggestion to apply for the fellowship that funded my work and allowed me to be a PhD student at WVU.

I do not think any graduate student can go through this journey without the understanding and support of others and I would like to thank all the past and present graduate students and post-docs I have met along the way. My time here definitely would not have been the same without Kellie Branson, Stephanie Lewis, Justin Lopez, Jackie Hand, and Brian Glover. I owe a special thanks to John Boney for showing me how to make feed for my sheep and to Crista Crawford for keeping track of the sheep, feeding them for me, and always helping with sample collection. A huge thanks to Dr. Bowdridge's lab group (Dr. Javier Garza, Dr. Jesica Jacobs, Elizabeth Shepherd, Brynnan Russ, Denzel Middleton, Taylor Harrison) for help with sample collection, for help in the lab as well as sharing your lab space and equipment with me, and for being great office roomates this last year. Lastly, I would like to thank the members of my own lab group, Hoda Nikpour, Melissa Wise, and Molly Potter, for all of their help on the farm, and Dr. Elizabeth Bowdridge who was there every step of the way from me learning how to work with sheep to learning new lab techniques.

Finally, I would never have made it this far without the support of my family, especially my

parents who always believed I could accomplish anything I wanted. And to my husband who is probably tired of me still being in school but has stuck with me through this journey anyway. 


\section{Table of Contents}

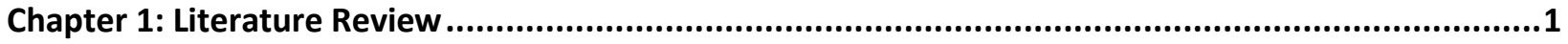

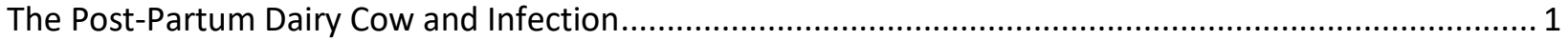

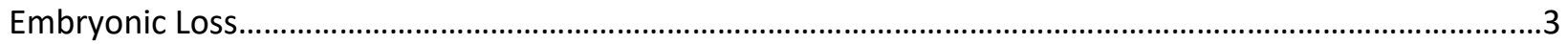

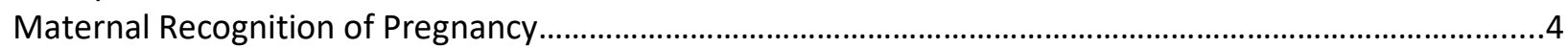

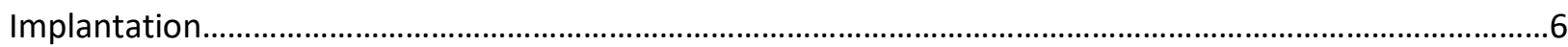

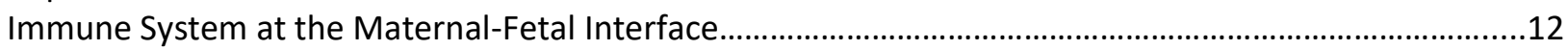

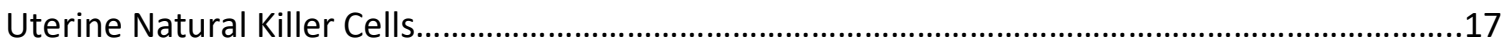

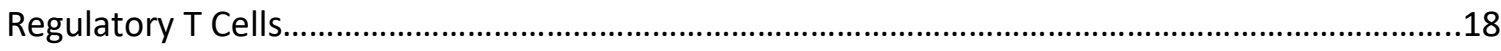

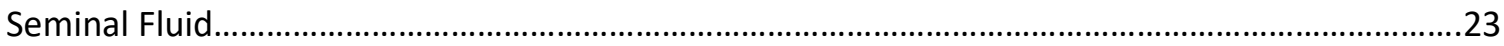

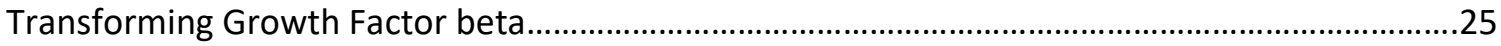

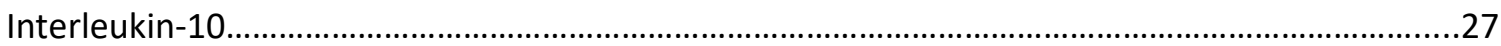

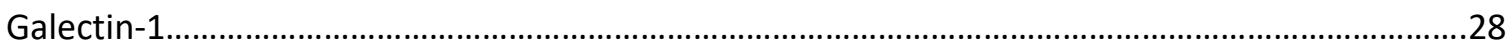

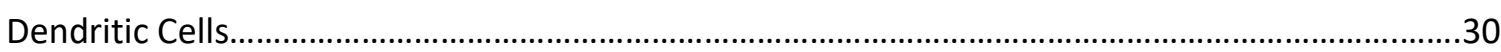

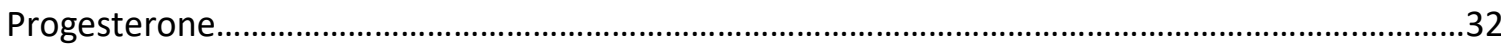

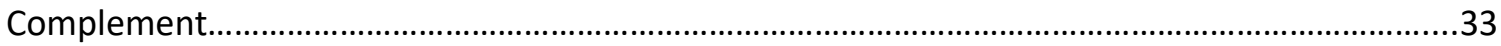

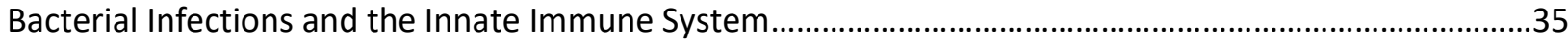

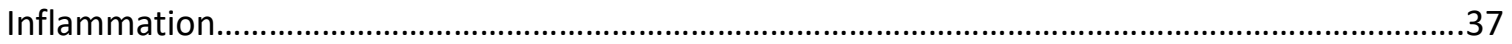

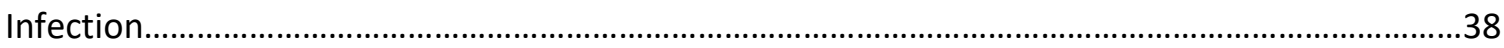

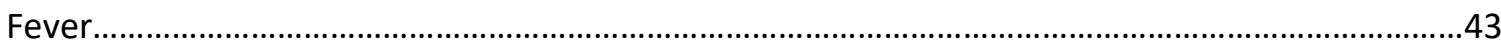

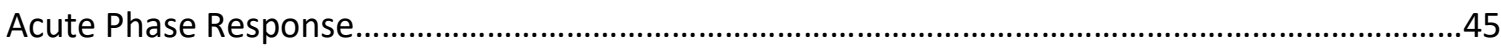

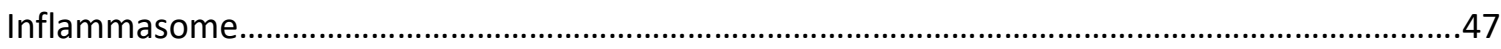

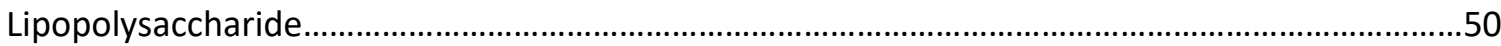

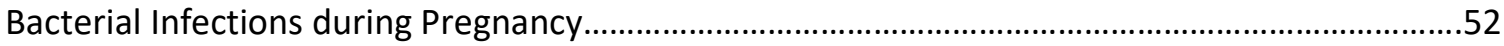

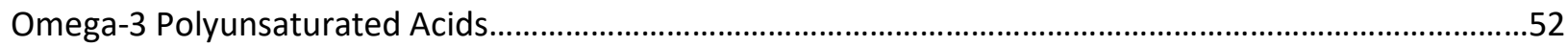

Resolution of Inflammation and Specialized Pro-Resolving Mediators...........................................53

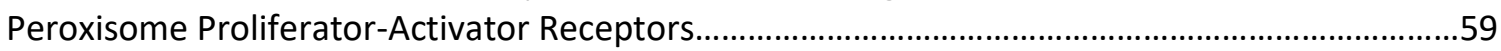

Dietary Supplementation with Omega-3 Polyunsaturated Fatty Acids...........................................62

Omega-3 Polyunsaturated Fatty Acids and Benefits to Reproduction............................................69

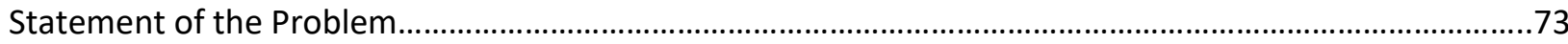

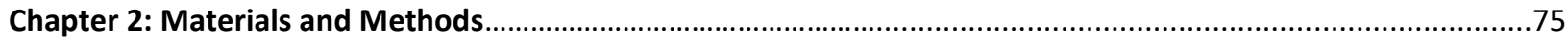

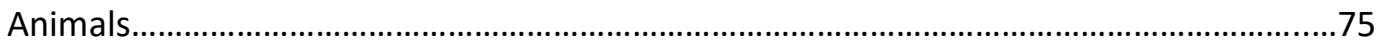

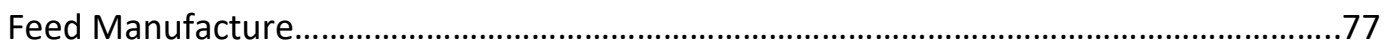

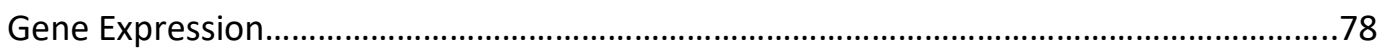

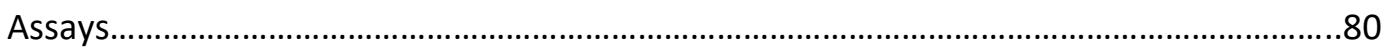

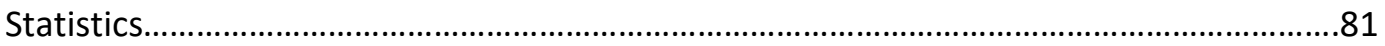

Chapter 3: Effects of Dietary Omega-3 Polyunsaturated Fatty Acids on LPS Challenge in Early Pregnant Dorset

Ewes

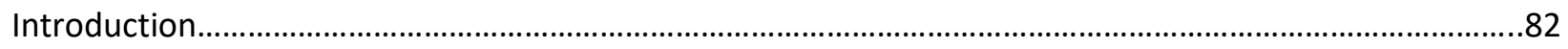

Trial 1

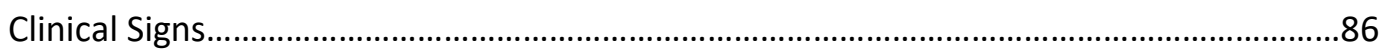

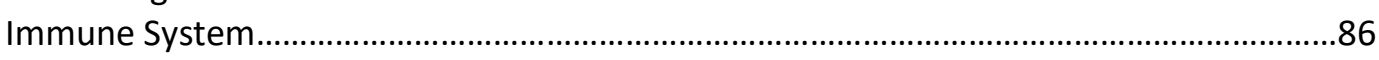

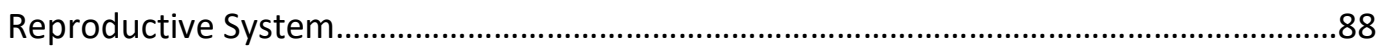

Trial 2

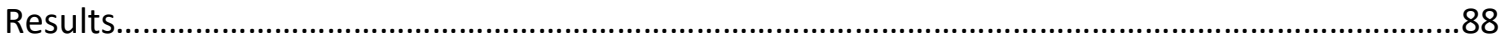




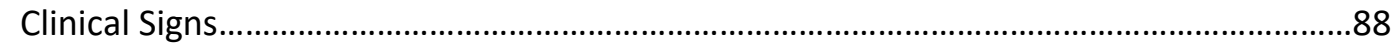

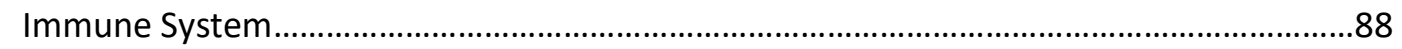

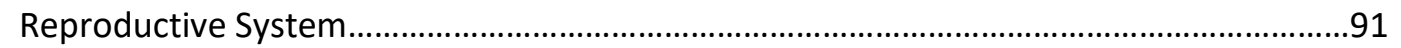

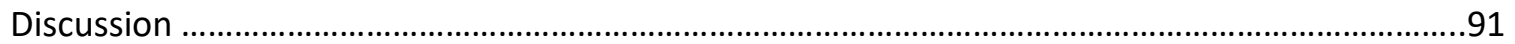

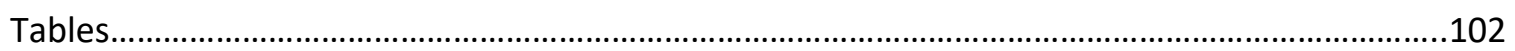

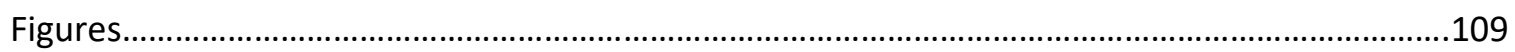

Chapter 4: Effect of Ovine Breed on the LPS Response in Early Pregnant Ewes...................................121

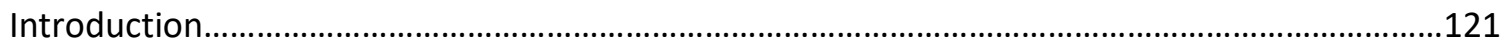

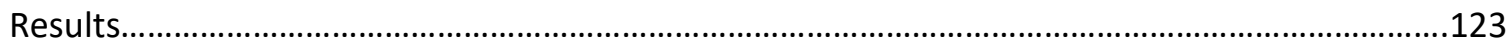

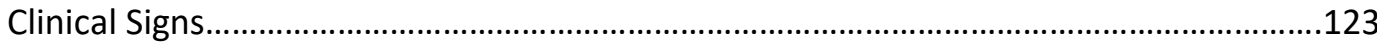

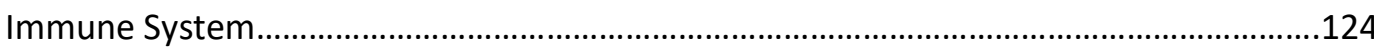

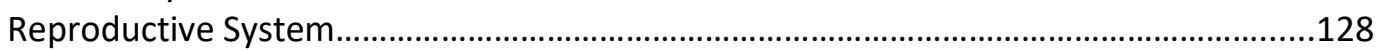

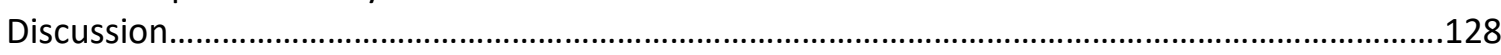

Table

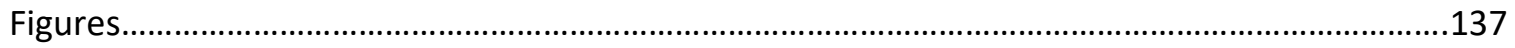

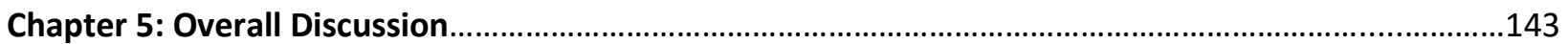

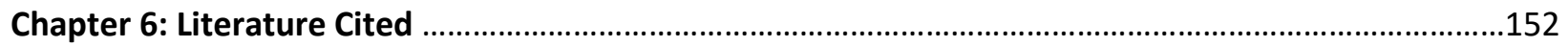




\section{List of Tables and Figures}

Table 1: Analysis of whole flaxseed used for trial 1 of the omega-3 PUFA study (Spring 2015)........102

Table 2: Composition of diets fed to ewes in trail 1 of the omega-3 PUFA study......................102

Table 3: Results from feed analysis conducted on samples of the prepared diets used in trial 1 of the

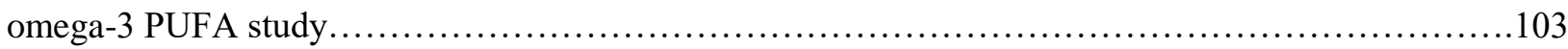

Table 4: Primer sequences of the housekeeping gene and genes of interest........................ 104

Table 5: Analysis of the whole flaxseed used for trial 2 of the omega-3 PUFA study (Fall 2015)...........104

Table 6: Composition of diets fed to ewes in trial 2 of the omega-3 PUFA study........................105

Table 7: Results from feed analysis conducted on samples of the prepared diets used in trial 2 of the

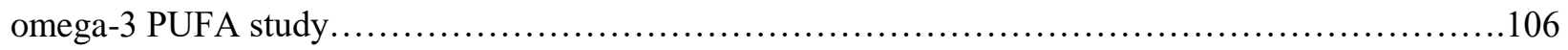

Table 8: Fold change in cyclooxygenase 2, interleukin-10, nuclear factor kappa B, peroxisome proliferator-activated receptor gamma, and tumor necrosis alpha expression compared to the CP group in

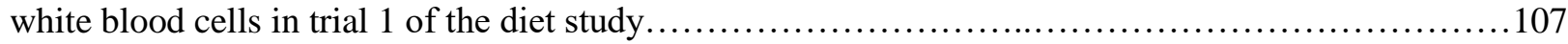

Table 9: Fold change in interferon gamma, interleukin-6, interleukin-10, nuclear factor kappa B, and tumor necrosis alpha gene expression to LPS or PBS in early pregnant ewes fed a whole flaxseed or

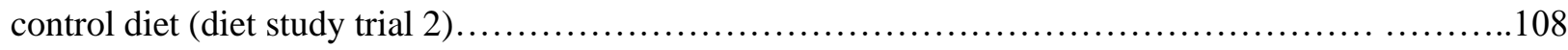

Table 10: Fold change in nuclear factor kappa B and tumor necrosis alpha gene expression to LPS or PBS in early pregnant Dorset and Suffolk ewes............................................... 137

Table 11: Summary of p-values across the three studies for main effects and interactions...............149

Table 12: Abbreviations used in dissertation.....................................................

Figure 1: Timeline outlining the steps of the three studies (Spring 2015) ...........................77

Figure 2: Hourly response in rectal temperature to LPS or PBS in early pregnant ewes fed a whole

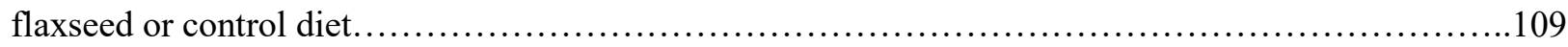

Figure 3: Frequency of sickness symptoms displayed by flaxseed and control ewes receiving either LPS

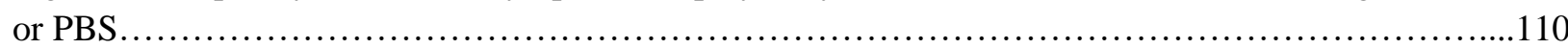
Figure 4: Hourly response in white blood cells to LPS or PBS in early pregnant ewes fed a whole flaxseed or control diet from 0-12 hours (a) and 0-5 hours (b) post treatment............................. 110 Figure 5: Plasma concentrations of the acute phase proteins (a) haptoglobin and (b) serum amyloid A..111

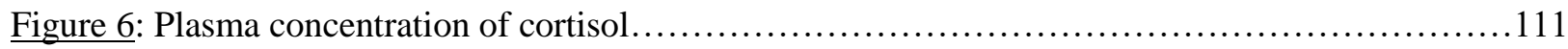
Figure 7: Fold change in gene expression of pro-inflammatory factors measured in white blood cells. A: CXCL8, B: IL-6, C: IFN $\gamma$, D: TLR4, E: C3 Figure 8: Fold change in gene expression of anti-inflammatory factors measured in white blood cells. A: MRC1, B: SOD2, C: Foxp3 ..........................................................113

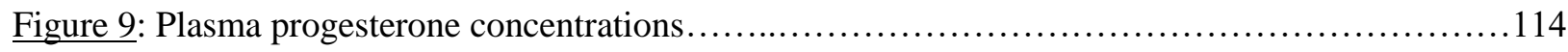
Figure 10: Neutral detergent fiber (a) and non-fiber carbohydrate (b) in samples of the prepared diets used in trial 1 of the $\omega-3$ PUFA study......................................................... 114 Figure 11: Timeline outlining the steps in the breed effects and the omega-3 PUFA effect on LPS

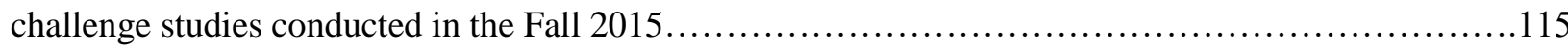
Figure 12: Rectal temperature to LPS or PBS in early pregnant ewes fed a whole flaxseed or control diet. A: Hourly response, B: Average for the first six hours. 
Figure 13: Frequency of sickness symptoms displayed by flaxseed and control diet fed ewes receiving

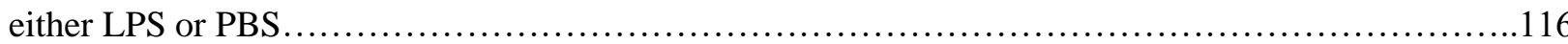

Figure 14: Response in white blood cells to LPS or PBS in early pregnant ewes fed a whole flaxseed or

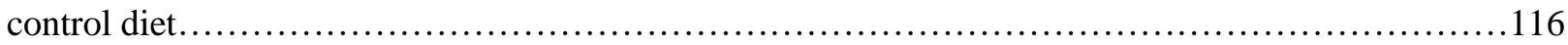
Figure 15: Response in the acute phase proteins (a) haptoglobin and (b) serum amyloid A to LPS or PBS in early pregnant ewes fed a whole flaxseed or control diet from 0-24 hours post treatment..... 117 Figure 16: Cortisol response to LPS or PBS in early pregnant ewes fed a whole flaxseed or control diet

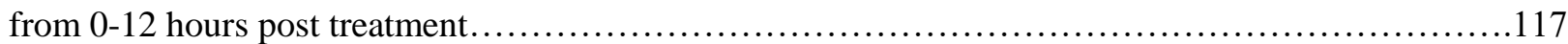
Figure 17: Fold change in gene expression of pro-inflammatory factors measured in white blood cells. A: CXCL8, B: C3, C: TLR4, D: COX2 118 Figure 18: Fold change in gene expression of anti-inflammatory factors measured in white blood cells. A: MRC1, B: SOD2, C: PPARy, D: Foxp3, E: TGF $\beta$. 119

Figure 19: Plasma concentration of progesterone on day $25 / 26$ post coitus in ewes fed flaxseed or a control diet and administered LPS or PBS on day $5 / 6$ post coitus Figure 20: Neutral detergent fiber (a) and non-fiber carbohydrate (b) in samples of the prepared diets used in trial 2 of the $\omega$-3 PUFA study.

Figure 21: Response in rectal temperature to LPS or PBS in early pregnant Dorset and Suffolk ewes. A: hourly response, B: hourly change from hour 0 .

Figure 22: Frequency of sickness symptoms displayed by Suffolk and Dorset ewes receiving either LPS or PBS.

Figure 23: Response of white blood cells to LPS or PBS in early pregnant Dorset and Suffolk ewes from hours $0-6$ post treatment. 138

Figure 24: Response of acute phase proteins to LPS or PBS in early pregnant Suffolk and Dorset ewes from 0-24 hours post treatment. A: haptoglobin, B: serum amyloid A...........................139 Figure 25: Cortisol response to LPS or PBS in early pregnant Dorset and Suffolk ewes from 0-12 hours post treatment.

Figure 26: Fold change in gene expression of pro-inflammatory factors measured in white blood cells. A:

CXCL8, B: IL-6, C: IFN $\gamma$, D: TLR4, E: COX2, F: C3

Figure 27: Fold change in gene expression of anti-inflammatory factors measured in white blood cells. A:

MRC1, B: IL-10, C: TGF $\beta$, D: SOD2, E: PPAR $\gamma$, F: Foxp3.

Figure 28: Plasma concentration of progesterone on day 25/26 post coitus in Dorset and Suffolk ewes administered LPS or PBS on day 5/6 post coitus.

Figure 29: Analysis Examining (a) whether a ewe was found to be pregnant or open by ultrasound on $25 / 26$ days post coitus and (b) whether or not a ewe lambed as a result of the mating that took place before the experiment was contingent on experimental group. 
Table 12: Abbreviations used in dissertation

\begin{tabular}{|c|c|c|c|c|c|}
\hline Acid Detergent Fiber & $\mathrm{ADF}$ & Haptoglobin & $\mathrm{Hp}$ & $\begin{array}{l}\text { Pathogen Associated Molecular } \\
\text { Pattern }\end{array}$ & PAMP \\
\hline Arachidonic Acid & AA & $\begin{array}{l}\text { 11-beta-hydroxysteroid } \\
\text { dehydrogenase type I }\end{array}$ & $\begin{array}{l}\text { HSD11 } \\
\text { B1 }\end{array}$ & $\begin{array}{l}\text { Peripheral Blood Mononuclear } \\
\text { Cell }\end{array}$ & PBMC \\
\hline Artificial Insemination & $\mathrm{AI}$ & $\begin{array}{l}\text { Intracellular Adhesion } \\
\text { Molecule } 1\end{array}$ & ICAM1 & $\begin{array}{l}\text { Programmed Death } \\
\text { Receptor/Ligand }\end{array}$ & $\begin{array}{l}\text { PD-1/ } \\
\text { PD-L1 }\end{array}$ \\
\hline $\begin{array}{l}\text { Alpha-Linolenic Acid } \\
(18: 3 n-3)\end{array}$ & ALA & Indoleamine 2,3-dioxygenase & IDO & Prostaglandins & PGs \\
\hline Antigen Presenting Cell & APC & Interferon gamma & IFN $\gamma$ & Prostaglandin E2 & $\mathrm{PGE}_{2}$ \\
\hline Acute Phase Proteins & APP & Interferon tau & IFN $\tau$ & Prostaglandin F2alpha & $\mathrm{PGF}_{2 \alpha}$ \\
\hline Acute Phase Response & APR & Interleukins & IL-\# & Pro-Inflammatory Cytokine & PIC \\
\hline$\beta$-hydroxybutyrate & BHB & IL-1 receptor antagonist & IL-1ra & Pro-Inflammatory Mediator & PIM \\
\hline $\begin{array}{l}\text { Bone Marrow Derived } \\
\text { Dendritic Cells }\end{array}$ & $\begin{array}{l}\text { BMD } \\
\text { C }\end{array}$ & $\begin{array}{l}\text { Interferon Inducible Protein } \\
10\end{array}$ & IP-10 & Phospholipase $\mathrm{A}_{2}$ & $\mathrm{PLA}_{2}$ \\
\hline Complement Fragments & $\mathrm{C} \#$ & Luminal Epithelia & LE & Polymorphonuclear Neutrophils & PMN \\
\hline Chemokine Receptor & $\begin{array}{l}\text { CCR } \\
\#\end{array}$ & Leukemia Inhibitory Factor & LIF & $\begin{array}{l}\text { Peroxisome Proliferator- } \\
\text { Activated Receptor gamma }\end{array}$ & PPAR $\gamma$ \\
\hline Chemokine & $\begin{array}{l}\mathrm{CCL} \\
\# \\
\text { CXC } \\
\text { L\# }\end{array}$ & Lipoxygenase & LOX & Pattern Recognition Receptor & PRR \\
\hline Corpus Luteum & $\mathrm{CL}$ & Lipopolysaccharide & LPS & Prostaglandin Synthase & PTGS \\
\hline $\begin{array}{l}\text { Complement receptor } \\
\text { 1-related gene/protein y }\end{array}$ & Crry & Leukotrienes & LTs & Poly Unsaturated Fatty Acids & PUFAs \\
\hline Crude Protein & $\mathrm{CP}$ & Leukotriene $\mathrm{B}_{4}$ & $\mathrm{LTB}_{4}$ & $\begin{array}{l}\text { Regulates on activated normal } \\
\mathrm{T} \text { cells where it is expressed } \\
\text { and secreted }\end{array}$ & $\begin{array}{l}\text { RANTE } \\
\mathrm{S}\end{array}$ \\
\hline $\begin{array}{l}\text { Cytotoxic T } \\
\text { Lymphocyte Antigen } 4\end{array}$ & $\begin{array}{l}\text { CTL } \\
\text { A4 }\end{array}$ & Lipoxins & LXs & Resolvin D1 & RvD1 \\
\hline Cyclooxygenase & COX & Lipoxin A4 & $\mathrm{LXA}_{4}$ & Resolvin E1 & RvE1 \\
\hline $\begin{array}{l}\text { Damaged Associated } \\
\text { Molecular pattern }\end{array}$ & $\begin{array}{l}\text { DA } \\
\text { MP }\end{array}$ & Macrophage & $\mathrm{M} \Phi$ & Reactive Oxygen Species & ROS \\
\hline Dendritic Cell & $\overline{\mathrm{DC}}$ & Matrix Metalloproteinase & MMP & $\begin{array}{l}\text { Suppressor of Cytokine } \\
\text { Signaling } 3\end{array}$ & SOCS3 \\
\hline Dry Matter Intake & $\overline{\text { DMI }}$ & $\begin{array}{l}\text { Monocyte Chemoattractant } \\
\text { Protein } 1\end{array}$ & MCP-1 & $\begin{array}{l}\text { Special Pro-Resolving } \\
\text { Mediators }\end{array}$ & SPMs \\
\hline $\begin{array}{l}\text { Docosahexaenoic Acid } \\
(22: 6 n-3)\end{array}$ & DHA & Mucin 1 & MUC-1 & Serum Amyloid A & SAA \\
\hline $\begin{array}{l}\text { Eicosapentaenoic Acid } \\
(20: 5 n-3)\end{array}$ & EPA & Negative Energy Balance & NEB & $\begin{array}{l}\text { Signal Transducer and } \\
\text { Activator of Transcription }\end{array}$ & STAT \\
\hline Estrogen & $\mathrm{E}_{2}$ & Neutral Detergent Fiber & NDF & T Cell Receptor & TCR \\
\hline Estrogen Receptor & ESR & Non-Esterified Fatty Acid & NEFA & $\begin{array}{l}\text { Transforming Growth Factor } \\
\text { Beta }\end{array}$ & TGF $\beta$ \\
\hline Fatty Acids & FAs & Natural Killer Cell & NK cell & $\begin{array}{l}\text { Induced T Regulatory Type } 1 \\
\text { Cells }\end{array}$ & $\operatorname{Tr} 1$ \\
\hline Galectin-1 & Gal-1 & Nitric Oxide & $\mathrm{NO}$ & Toll Like Receptors & TLR \\
\hline Glandular Epithelia & GE & Organic Matter & OM & Regulatory T Cells & Treg \\
\hline Glucocorticoid & $\mathrm{GC}$ & Oxytocin Receptor & OXTR & Uterine Natural Killer Cell & uNK \\
\hline $\begin{array}{l}\text { Granulocyte-Colony } \\
\text { Stimulating Factor }\end{array}$ & $\begin{array}{l}\text { G- } \\
\text { CSF }\end{array}$ & Progesterone & $\mathrm{P}_{4}$ & $\begin{array}{l}\text { Vascular Endothelial Growth } \\
\text { Factor }\end{array}$ & VEGF \\
\hline $\begin{array}{l}\text { Hypothalamic- } \\
\text { pituitary-adrenal axis }\end{array}$ & $\begin{array}{l}\text { HPA } \\
\text { axis }\end{array}$ & Platelet Activating Factor & PAF & Volatile Fatty Acid & VFA \\
\hline
\end{tabular}




\section{Chapter 1. Literature Review \\ The Post-Partum Dairy Cow and Infection}

High producing dairy cows are under considerable metabolic stress as they experience a large increase in nutritional and energy requirements for milk production (peaks 4-8 weeks post-partum) after calving. The increased energy requirements are only partially offset by increased feed consumption (due to limitations in intake and appetite), and the remainder is met by mobilization of body reserves (Bauman and Currie, 1980; Evans and Walsh, 2012). This often leads to a state of severe negative energy balance (NEB) characterized by loss in body condition, low blood glucose levels, elevated non-esterified fatty acid (NEFA) levels, and elevated ketone bodies such as $\beta$-hydroxybutyrate (BHB). These changes indicate increased lipid mobilization and fatty acid oxidation. This metabolic environment has negative effects on immune cells with the resulting immunosuppressive environment making cows more susceptible to mammary (mastitis) and uterine (endometritis) infections with less ability to clear the infection (Mallard et al., 1998; Hammon et al., 2006; Wathes et al., 2009; Morris, 2014). The immune system uses energy produced from oxidative phosphorylation, therefore, being in a state of ketosis impairs immune system function. For example, in response to lipopolysaccharide (LPS) the mitochondrial activity of macrophages exposed to BHB in vitro was decreased and they produced less reactive oxygen species (ROS), which are used to eliminate bacteria. However, with the addition of eicosapentaenoic acid (EPA), ROS production by macrophages in response to LPS increased, while docosahexaenoic acid (DHA) had no effect (Morris, 2014).

In selecting dairy cows for high milk production, a global decline in fertility and an increase in the incidence of infectious diseases has occurred (Uribe et al., 1995; Thatcher et al., 2010; Walsh et al., 2011). Cows undergo metabolic adaptations to NEB during the first weeks of lactation (Suriyasathaporn et al., 2000), which will impair immune system function making them prone to infectious diseases and, subsequently, impacting fertility (Hogan et al., 1989; Goff, 2006; Evans and Walsh, 2012). A number of factors contribute to poor fertility in high-producing dairy cows including short duration and low intensity of oestrous due to low circulating estrogen $\left(\mathrm{E}_{2}\right)$ concentrations and increased metabolic clearance rate of steroids related to liver blood flow resulting in lowered peripheral concentrations of progesterone $\left(\mathrm{P}_{4}\right)$ and $\mathrm{E}_{2}$. Lowered concentrations of $\mathrm{P}_{4}$ may be unable to prevent prostaglandin $\mathrm{F}_{2 \alpha}\left(\mathrm{PGF}_{2}\right)$ release and luteolysis and may affect the uterine secretion of proteins and growth factors needed for early embryo development (Diskin and Morris, 2008). The most frequent factor contributing to low fertility, however, is high embryonic mortality. Embryonic loss can be due to poor oocyte quality resulting from the adverse 
metabolic environment or due to poor uterine environment influenced by uterine infection and low circulating $\mathrm{P}_{4}$ concentrations (Evans and Walsh, 2012).

For optimum economic return, farmers need to rebreed cows within 2-3 months of calving, however, poor fertility is a major problem in the dairy industry with metabolic stress and reproductive issues being linked (Roche, 2006). To achieve a 365 day calving interval with a 282 day gestation, breeding usually commences 60 days post-partum and the cow needs to conceive by 83 days postpartum. For the cow to become and stay pregnant any uterine infections need to be resolved; she needs to recover from post-partum NEB; and she needs to resume normal estrous cycles (Walsh et al., 2011). Cows that suffer from metabolic disorders (acidosis, fatty liver disease, displaced abomasum, hypocalcaemia, hypomagnesaemia, ketosis) in the peri-parturient period are more likely to have increased incidence of mastitis, lameness, and endometritis (Roche, 2006) and reduced pregnancy success.

After parturition, the uterus undergoes remodeling to get back to the pre-pregnant state including a reduction in size, removal of cellular debris, and restoration of normal architecture (Gier and Marion, 1968; Leslie, 1983; Sheldon and Dobson, 2004). It is normal to have uterine contamination with bacteria at parturition or during the first few days postpartum with $80-100 \%$ of cows having bacterial contamination in the first two weeks after calving (Foldi et al., 2006; Lewis, 1997; Sheldon et al., 2006). The most prevalent bacteria in the post-partum uterus are Escherichia coli and Arcanobacterium pyogenes, but other common bacteria include Fusobacterium necrophorum, Prevotella melaninogenicus, and Proteus species, which are associated with increased endometrial inflammation and purulent vaginal mucus (Bondurant, 1999; Sheldon et al., 2009a). Initially, mucosal defense and the innate immune system are responsible for dealing with the pathogens and eliminating the contamination, which is usually cleared within $\sim 3$ weeks of calving with complete involution of the uterus and cervix within 4-6 weeks. However, lactating dairy cows are predisposed to reduced immune competence, and the immune system of cows that cannot clear the bacteria can become overwhelmed resulting in uterine disease (metritis) (King et al., 2003; Sheldon et al., 2006; LeBlanc, 2008; Walsh et al., 2011). Metritis can develop within 3 weeks postpartum and in about $20 \%$ of cows the pathogenic bacteria will persist in the uterus for longer than 3 weeks, the consequence being endometritis (Sheldon et al., 2009a). Endometritis is caused by chronic bacterial infection in the uterus causing uterine inflammation without systemic illness. The condition is characterized by disruption of the endometrial epithelium, infiltration of inflammatory cells and lymphocytes, stromal edema, and vascular congestion (Bondurant, 1999). Approximately 15-20\% of cows have clinical endometritis at 4-6 weeks postpartum, and 30-35\% will have subclinical endometritis between 4 and 9 weeks postpartum (LeBlanc, 2008). Events associated with parturition can influence 
subsequent infection: cows having twins, dystocia, a stillbirth, or retained fetal membranes have increased the risk of infection (LeBlanc, 2008).

Uterine infections are characterized by extensive leukocyte infiltration of the endometrium and chronic inflammation (Foldi et al., 2006; Sheldon et al., 2006) resulting in longer intervals to conception which increases the likelihood of culling (Lewis, 1997; Fourichon et al., 2000; Gilbert et al., 2005; Wise, 2016). Indeed, being diagnosed with clinical mastitis between artificial insemination (AI) and pregnancy confirmation (45 days post-AI; 14 days after ultrasound diagnosis) increased the odds of pregnancy loss (odds ratio 2.80 clinical mastitis versus no symptoms of disease) in lactating Holstein cows (Chebel et al., 2004). Several studies have found that the type of bacteria (gram negative or positive) did not affect the reproductive parameters evaluated but reported differences when comparing cows without clinical mastitis, cows that had mastitis before first AI, and cows that got mastitis after pregnancy diagnosis. The group of cows that were diagnosed between first AI and pregnancy confirmation had the lowest percent of conception at first AI, the lowest pregnancy rate at 320 DIM, the highest average number of services per conception, and the highest number of days open, showing that infection during early pregnancy is detrimental to reproductive performance (Barker et al., 1998; Santos et al., 2004b). Progesterone reduces cervical mucus production, prevents uterine contractility (Rodriguez-Martinez et al., 1987) and counteracts estrogen's effects on immune protective responses of the reproductive tract such as antibody secretion and increased activity of antigen presenting cells in the uterus (Wira et al., 1995; Bondurant, 1999). Therefore, the presence of a CL and $\mathrm{P}_{4}$ production makes the uterus susceptible to infection and hinders the ability of the immune system to clear pathogens, and the presence of bacteria can result in a clinical infection after ovulation with the production of $\mathrm{P}_{4}$.

\section{Embryonic Loss}

In the cow, embryo loss between fertilization and day 24 of gestation is considered early embryo mortality, loss between day 25 and 45 (embryonic differentiation is completed) is considered late embryo mortality, and losses between day 46 and parturition are considered fetal mortality (Committee on Bovine Reproductive Nomenclature, 1972). The peri-implantation period is a critical time in determining if pregnancy will be successful. As milk production has increased over the last 50 years in dairy cattle, fertility has decreased (Diskin et al., 2006; Sartori et al., 2010; Walsh et al., 2011). In lactating cows, first service conception rates are $-32 \%$, whereas in heifers the rate is over $50 \%$. With a fertilization rate over $80 \%$, the low conception rate does not seem to be due to problems with fertilization (Sartori et al., 2010). The low conception rate is instead related to high rates of embryonic loss with 70-80\% of embryos being lost during the first 3 weeks after insemination (Inskeep and Dailey, 2005; Diskin et al., 2006; Diskin and 
Morris, 2008; Hansen, 2011b). This has a huge impact on milk production efficiency and profitability (Thatcher et al., 2011; Evans and Walsh, 2012).

During the first 7 days after insemination the embryo develops into a blastocyst dependent on its inherent ability to develop, which is associated with oocyte quality, sperm quality, and timing of fertilization (Evans and Walsh, 2012). The uterine environment also impacts early embryonic development, and persistent issues in the post-partum uterus can make the environment suboptimal (Evans and Walsh, 2012). In support of this idea, Rizos et al (2010) conducted a zygote transfer (to the oviducts at day 2) experiment that showed that in post-partum lactating Holstein-Friesian cows were less able to support very early embryo development compared to Holstein-Friesian heifers. They suggested that the reproductive tract of the lactating dairy cow is a less suitable environment for early embryo development compared to the tract of a heifer. It is estimated that by day 7 of gestation only $45-55 \%$ of inseminated lactating cows are pregnant whereas $\sim 75 \%$ of inseminated heifers are pregnant (Sartori et al., 2010; Walsh et al., 2011).

The presence of pathogenic bacteria can compromise the uterine environment causing embryonic death and abortion (Sheldon et al., 2004; 2006). The majority of cows have uterine bacterial contamination post-partum, but clinical infection does not always result. (Sheldon et al., 2006). Even without clinical infection, inflammation induced by the bacteria can perturb embryo survival (Hansen et al., 2004). The majority of microbes enter the uterus by ascending through the reproductive tract after being introduced following parturition, abortion, natural service, or AI. Endometrial cells have pattern recognition receptors (PRRs) that recognize pathogen associated molecular patterns (PAMPs) such as unmethylated DNA, lipids, and LPS. Following interaction of a PRR with its PAMP, antimicrobial peptides, pro-inflammatory cytokines, and chemokines are released resulting in activation and mobilization of immune cells and an inflammatory environment (Akira et al., 2006; Herath et al., 2006; Sheldon et al., 2009a; Cronin et al., 2012).

Demand for milk and milk production per dairy cow are expected to continue to increase. In order to have dairy cows capable of high milk production and efficient reproduction, it is necessary to optimize health, nutrition, and breeding management strategies (Walsh et al., 2011). Strategies that minimize embryonic loss will improve the reproductive efficiency of ruminants.

\section{Maternal Recognition of Pregnancy}

To establish a pregnancy after conception, three events must occur: pregnancy recognition signaling, implantation, and placentation (Guillomot et al., 1995; Spencer et al 2004). In ruminants, endometrial function in early pregnancy is regulated mostly by $\mathrm{P}_{4}$ produced by the CL and by conceptus 
secreted factors like IFN- $\tau$ produced by the mononuclear trophectoderm cells (Spencer et al., 2004b; Spencer et al., 2008; Bazer et al., 2010). Progesterone from the CL acts on the uterus to stimulate preimplantation blastocyst growth and conceptus elongation in ruminants (Spencer et al., 2004b), which are critical for production of IFN- $\tau$ and prostaglandins (PGs) by the trophectoderm (Spencer et al., 2007). Progesterone production is required for the duration of pregnancy; in some species the placenta will eventually take over production while in others, the CL is solely responsible. To maintain CL function past its normal lifespan during a luteal phase the conceptus must produce a hormone that acts on the uterus and/or CL (Bazer et al., 2013).

In cyclic sheep, the uterus produces luteolytic pulses of $\mathrm{PGF}_{2 \alpha}$ on days 15-16 of the cycle to regress the CL. In ruminants, pregnancy is recognized by the dam through production of IFN- $\tau$ by the conceptus (Helmer et al., 1989; Bazer, 2013). The IFN- $\tau$ gene arose in ruminants through duplication of the IFN- $\omega$ gene (Roberts et al., 1998) and through evolution the promotor region lost its viral control elements while gaining two regions that make its expression trophectoderm-specific (Hansen et al., 1991; Ezashi et al., 1998; Roberts et al., 2003). In one of these acquired regions is an enhancer element for the transcription factors Ets-2 and AP-1. The transient expression of IFN- $\tau$ is regulated by Ets-2, which is activated in response to growth factors and cytokines released by the maternal endometrium perhaps in response to $\mathrm{P}_{4}$ (Ezashi et al., 1998; Roberts et al., 2003). Secretion of IFN- $\tau$ begins at the blastocyst stage, peaks during elongation, and is terminated upon trophectoderm attachment to the uterine endometrium in ewes (maximum production on days 14-16; production stops on days 21-25) (Guillomot et al., 1990; Bazer, 1992; Martal et al., 1998).

In sheep, pregnancy recognition occurs on day 13 post-mating, and communication between the endometrium and conceptus continues during implantation and synepitheliochorial placentation, which begins on days 15-16 and is completed between days 50-60 (Wimsatt, 1950; Guillomot, 1995). Binding to receptors in the endometrium (Han et al., 1997), IFN- $\tau$ acts to suppress transcription of the estrogen receptor alpha gene (ESRI) stopping $\mathrm{E}_{2}$-induced expression of the oxytocin receptor $(O X T R)$ in the uterine luminal and superficial glandular epithelia. This prevents the oxytocin dependent release of luteolytic pulses of $\mathrm{PGF}_{2 \alpha}$ and regression of the CL, allowing for continued $\mathrm{P}_{4}$ production by the CL (Helmer et al., 1989; Bazer et al., 2008; Bazer, 2013). Progesterone is the unequivocal hormone of pregnancy as it influences uterine functions needed for early embryonic development, implantation, placentation, and placental and fetal development (Spencer and Bazer, 1996; Martal et al., 1998; Spencer and Bazer, 2004; Roberts et al., 2008; Bazer et al., 2010). During implantation the uterus grows and remodels, placentomes develop in the caruncular area of the endometrium, angiogenesis and vascular remodeling occur, and intercaruncular endometrial glands increase in size (Wimsatt, 1950). Endometrial epithelial gene 
expression and function for conceptus elongation in early pregnancy are regulated not only by ovarian, CL-produced $\mathrm{P}_{4}$ but also by cortisol, PGs, and IFN- $\tau$ (Spencer et al., 2004b; Spencer et al., 2008; Bazer et al., 2010; Dorniak et al., 2011; Dorniak et al., 2012a). Endometrial glands are responsible for the production of histotroph consisting of growth factors, cytokines, lymphokines, enzymes, hormones, and transport proteins, which are needed for conceptus survival and development, fetal and placental growth, and the onset of pregnancy recognition signals (Roberts and Bazer, 1988; Gray et al., 2001a, b).

\section{Implantation}

The success of implantation affects ongoing pregnancy, and any complications at this stage will contribute to pregnancy complications and embryonic loss (Song et al., 2002; Dey, 2005). Blastocyst implantation in the uterus is required for delivery of nutrients and gas exchange between the developing offspring and dam. Implantation involves shedding the zona pellucida (blastocyst hatching), blastocyst elongation, orientation of the blastocyst, apposition (trophectoderm becomes closely associated with the endometrial luminal epithelium (LE) without adhesion), attachment and adhesion between the trophectoderm and the uterine LE forming the placenta (Spencer et al 2004; Guillomot et al., 1995; Degrelle et al., 2005; Bazer et al., 2009a).

A sheep embryo enters the uterus at the morula stage on day 4 post-mating before developing into a blastocyst (inner cell mass and blastocoele surrounded by a monolayer of trophectoderm) by day 6 and hatching from the zona pellucida on day 8 to 9. The blastocyst then develops into an ovoid or tubular form by days 11 to 12 when it is then called a conceptus (embryo-fetus plus associated extraembryonic membranes) (Guillomot et al., 1995; Spencer et al 2004). The conceptus then elongates into a filamentous form on days 12 to 16 . During conceptus elongation the trophectoderm increases exponentially in length and weight (Wales and Cuneo, 1989), and this process requires substances secreted from the endometrial LE and glandular epithelia (GE) (Gray et al., 2001a; 2002). Blastocyst elongation is critical for

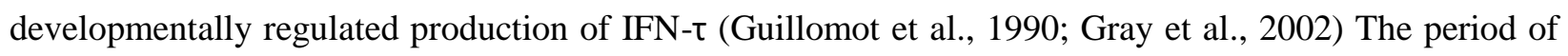
elongation also includes the start of extraembryonic membrane differentiation, gastrulation of the embryo, formation of the yolk sac and allantois, and cross-talk with the endometrium (Guillomot, 1995; Diskin and Morris, 2008). This is a critical time for successful pregnancy with over $70 \%$ of pregnancy failures associated with embryonic death during the early and late pre-implantation period (before d16 following breeding) (Diskin and Morris, 2008).

Ruminants have a bicornuate uterus with the endometrium having caruncles and intercaruncular areas. The caruncles are aglandular structures composed of LE and compact stroma that are distributed over the endometrial surface; they connect to the cotyledons (fetal part of placenta) to form placentomes. This type of superficial implantation is called synepitheliochorial placentation, and the placentomes have 
the role of delivery of nutrients and fetal-maternal gas exchange. The intercaruncular areas contain endometrial glands that produce histotroph, a mixture of nutrients, growth factors, enzymes, hormones and cytokines particularly important for the embryo before it has attached to the uterus (Spencer and Bazer, 2004; Spencer et al., 2004a).

In sheep, adhesion to the endometrial LE occurs on day 16 between the mononuclear trophoblast cells and the endometrial LE. Syncytia form from the fusion of trophoblast binucleate cells with the LE. The binucleate cells begin to differentiate in the trophoblast between days 14 and 16 before migrating and fusing with the endometrial LE to form syncytia that eventually cover the caruncular surface and help in formation of the placentome. Implantation involves loss of expression of the glycoprotein mucin1 (MUC1) (an antiadhesive protein) and expression of adhesion molecules by the trophoblast and endometrial LE (Spencer et al., 2004a), allowing for interdigitation of uterine microvilli with cytoplasmic projections on the trophoblast. Even in the intercaruncular regions, villi on the trophoblast interact with the endometrial LE; however, these projections disappear by day 20, and attachment is completed around day 22 (Boshier, 1969; Guillomot et al., 1981).

For the embryo to attach and implant in the uterus, the cell surface of the uterine epithelial cells undergo biochemical changes including changes to glycan structures. Through the secretion of leukemia inhibitory factor (LIF) and IL-1 $\beta$, macrophages (MФ) induce the expression of fucosyl transferases that increases the surface expression of fucosylated structures on uterine epithelial cells that are involved in trophoblast attachment (Jasper et al., 2011; Nakamura et al., 2012). Therefore, changes in the quantity or activity of uterine $\mathrm{M} \Phi$ could impair implantation.

Implantation will occur only between an implantation-competent blastocyst and a receptive uterus which occurs for a limited period of time known as the "window of implantation." The uterus will be unreceptive to a blastocyst before this time and become hostile to blastocyst survival after this receptive period (Yoshinaga, 1988; Dey, 2005). Therefore, anything that interferes with implantation during this "window of implantation" will cause embryonic loss. Synchronization between acquisition of implantation competency by the blastocyst and a receptive uterine endometrium is controlled by maternal hormones $\left(\mathrm{E}_{2}\right.$ and $\left.\mathrm{P}_{4}\right)$ along with locally produced signaling molecules that include cytokines, growth factors, lipid mediators, and transcription factors (Dey et al., 2004).

Lipid signaling has been shown to be critical during implantation. Knock out of cyclooxygenase2 (COX-2) in mice has resulted in implantation failure (Lim et al., 1997; Wang et al., 2004). In addition, cytoplasmic phospholipase $\mathrm{A}_{2 \alpha}\left(\mathrm{CPLA}_{2 \alpha}\right)$ null mice had a brief delay of the normal "window" of implantation leading to embryonic loss, retarded feto-placental development, and, post implantation 
retarded development. Exogenous $\mathrm{PGE}_{2}$ and $\mathrm{PGI}_{2}$, however, restores the normal timing of implantation (Song et al., 2002). PLA $2 \alpha$ is an enzyme that releases FAs from the second carbon group of glycerol; after catalytically hydrolyzing the bond, arachidonic acid (AA) is released which can then be turned into $\mathrm{PGH}_{2}$ by cyclooxygenases (the rate limiting enzyme in PG synthesis) followed by conversion into eicosanoids by specific enzymes (Smith et al., 2000). While COX1 is constitutively expressed, COX2 expression is induced by cytokines, growth factors, and mitogens and is responsible for increased PG production involved in inflammation, mitogenesis, angiogenesis, and differentiation (Smith et al., 2000). Implantation is an inflammatory process, therefore, a role for COX2 and PGs during implantation is logical.

Histotroph is derived mostly from transport and/or synthesis and secretion of substances by the endometrial LE and GE (Gray et al., 2001b). The substances (proteins, amino acids, saccharides, lipids, cytokines and growth factors) in histotroph control the elongation of the conceptus through effects on proliferation and migration of trophectoderm, and play a role in the attachment and adhesion to the endometrial LE (Bazer et al., 2010; Carter, 2012). In ewes, endometrial gland hyperplasia occurs between days 15 and 50 and is largely dependent on ovine placental lactogen secreted by binucleated trophoblast cells starting on day 16 (Wooding et al., 1992; Noel et al., 2003). Hypertrophic growth of the uterine glands then occurs after day 60 under the influence of ovine growth hormone of trophoblastic origin resulting is maximal histotroph production (Stewart et al., 2000; Noel et al., 2003). Histotroph is secreted in response to steroids, IFNt, ovine prolactin, and placentally expressed ovine growth hormone (Noel et al., 2003; Carter, 2012).

One component of histotroph, lipids, is an important component of cells and have both bioactive and structural properties (Ribeiro et al., 2016c). When Ribeiro et al (2016a) categorized recovered dairy cow conceptuses as ovoid ( 1 to $4 \mathrm{~mm}$ ), tubular (5 to $19 \mathrm{~mm}$ ), or filamentous (20 to $60 \mathrm{~mm}$ ) before comparing their transcriptomes, they determined that one of the major categories of differently expressed genes was lipid metabolism. As the conceptus transitioned from ovoid to tubular to filamentous, genes involved in lipid uptake, lipid droplet formation, activation and oxidation of FAs, biogenesis of peroxisomes, desaturation and elongation of FAs, mobilization of membrane phospholipids, biosynthesis of phospholipids and prostaglandins, and transport of prostaglandins/lipid metabolites became upregulated (Ribeiro et al., 2016a). The process of elongation involves rapid remodeling and proliferation of cells that need lipids for their membranes (cell and organelle). This lipid is supplied by the uptake of lipids from the uterine lumen and from de novo synthesis; therefore, genes related to the uptake of lipids from extracellular space (transporters) and genes involved in the synthesis and modification (elongases, desaturases) of FAs increase at the start of elongation (Ribeiro et al., 2016a). Lipid accumulates mainly in 
the LE and superficial GE and is mainly under the control of $\mathrm{P}_{4}$ (Brinsfield and Hawk, 1973) and is likely an important FA source for the conceptus. A reduced number of lipid droplets in endometrial epithelial cells of pregnant ewes indicates use of the lipids by the conceptus and uterus (Boshier et al., 1987). Not only are lipids used in the production of cells, but they are also an energy source, they serve as signaling molecules and as precursors of lipid derivatives like eicosanoids (Fernandis and Wenk, 2007; Ribeiro et al., 2016a).

Ribeiro et al (2016c) specifically pointed out that peroxisome proliferator-activated receptor gamma (PPAR $\gamma$ ) is likely to play an important role in elongation because they saw expression of PPAR $\gamma$ increase at the start of elongation; functional analysis selected PPAR $Y$ as an upstream regulator of transcriptome changes; and PPARY expression was correlated with gene expression of other genes involved in conceptus development and lipid metabolism (Ribeiro et al., 2016c). PPAR $\gamma$ is a receptor that functions as a transcription factor in several biological processes (Berger and Moller, 2002; Wieser et al., 2008) and functions in cell differentiation, uptake and metabolism of FAs, and vascularization in the placenta (Schaiff et al 2007; Fournier et al., 2007; Fournier et al 2011; Shalom-Barak et al., 2012). Brooks et al. (2015) conducted a loss-of-function study in sheep in which PPAR $\gamma$ was shown to regulate elongation. Without PPAR $\gamma$, conceptuses were growth-retarded and there was reduced secretion of IFN- $\tau$ and PGs, which affected endometrial physiology.

Cortisol, $\mathrm{P}_{4}, \mathrm{IFN}-\tau$, and $\mathrm{PGs}$ each have individual actions in addition to interactive and coordinated actions to regulate expression of genes in the endometrial epithelium related to elongation and implantation. Altering expression of these genes results in secretion or transport of substances from the endometrium into the uterine lumen that control conceptus elongation by affecting trophectoderm proliferation, migration, attachment, and adhesion (Dorniak et al., 2013a). During days 10 to 12 after the onset of estrus or mating in the ewe, $\mathrm{P}_{4}$ induces the expression of genes in the endometrial LE and superficial GE that encode intracellular enzymes (prostaglandin synthase 2: PTGS2, 11-betahydroxysteroid dehydrogenase type I: HSD11B1), secreted attachment and migration factors, secreted proteases, secreted proteases inhibitors, a secreted cell proliferation factor, glucose transporters, and a cationic amino acid transporter. In the endometrial GE, $\mathrm{P}_{4}$ induces expression of genes that encode for a secreted cell proliferation factor, a secreted adhesion protein, a glucose transporter, a regulator of calcium/phosphate homeostasis, and an immunomodulatory factor (Spencer et al., 2007; Bazer et al., 2010). The result of these gene changes induced by $P_{4}$ is an increase in specific amino acids, glucose, cytokines, and growth factors in histotroph that support blastocyst growth into an ovoid conceptus and elongation to form a filamentous conceptus (Bazer et a, 2010). 
IFN- $\tau$ inhibits the expression of ESR and OXTR in uterine LE and superficial GE, but it does not inhibit the expression of PTGS2 (Kim et al., 2003), the rate limiting enzyme in synthesis of PGs. Therefore, PGs can still be synthesized, just not in response to oxytocin. Cells of the bovine uterus are stimulated to produce PGE2 by IFN- $\tau$, and other type I IFNs stimulate production of phospholipase A2 (PLA2), providing the substrate for PTGS2, and synthesis of PGE2 and PGF in various cell types (Arosh et al., 2004; Bazer et al., 2013; Dorniak et al., 2013). In the ewe, PTGS2 expression is induced by ovarian $\mathrm{P}_{4}$ and appears between day 10 and 12 post-estrus and mating in the endometrial LE and sGE (Charpigny et al., 1997a; Simmons et al., 2010). Epithelial and stromal cells of the uterus secrete PGs that affect expression of genes critical for elongation and implantation of the ovine conceptus and the elongating conceptus synthesizes and secretes additional PGs that have paracrine effects on uterine cells, and autocrine effects on the conceptus. Receptors for $\mathrm{PGE}_{2}$ (PTGER2 and PTGER4) and PGF $2 \alpha$ (PTGFR) are expressed by the conceptus trophectoderm and the endometrium epithelia in the ovine uterus during early pregnancy (Dorniak et al., 2011). In addition, the PGI2 receptor (PGIR) and nuclear PG receptors (peroxisome proliferating and activating receptors (PPARs)) are expressed by the endometrial epithelia and by sheep conceptus during early pregnancy (Cammas et al., 2006).

Ovine conceptuses secrete 6-keto-PGF1 $\alpha$ (stable metabolite of PGI2), PGF, and PGE2 during the peri-implantation period that mediate, in part, the effects of IFN- $\tau$ and $\mathrm{P}_{4}$ to regulate endometrial functions for growth and development of the conceptus during this time (Charpigny et al., 1997b; Dorniak et al., 2011; Dorniak et al., 2012b; Bazer et al., 2013; Dorniak et al., 2013). Dorniak et al (2011) reported that giving a PTGS inhibitor from days 8-14 post-mating resulted in embryos that had not elongated by day 14 when they were flushed. When the PTGS inhibitor was given from days 10-14 post-estrus in cyclic ewes a decrease in the expression of $\mathrm{P}_{4}$-induced endometrial genes such as insulin growth factor binding protein-1 (IGFBP1) (mediate trophectoderm migration and attachment) and HSD11B1 (Simmons et al., 2009; 2010) was reported. When IFN- $\tau$ was infused, instead of the PTGS inhibitor, PTGS activity and PG levels in the uterine lumen increased. However, when IFN- $\tau$ was co-infused with the PTGS

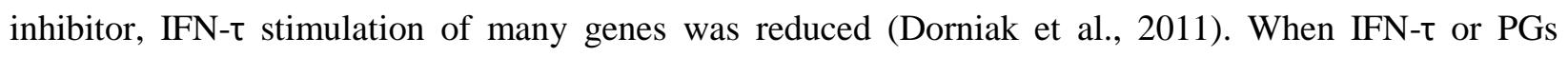
(PGE2, PGI2) were infused into the uteri from days 10-14 post-estrus, genes of glucose transporters increased and expression of genes thought to be involved in cell proliferation and migration, trophectoderm migration and attachment, and angiogenesis increased (Farmer et al., 2008; Simmons et al., 2009; Satterfield et al., 2009; Dorniak et al., 2012a). Apparently, PTGS derived prostaglandins are involved in mediating endometrial functions and changes in gene expression in response to IFN- $\tau$ and $\mathrm{P}_{4}$, which are critical for conceptus elongation (Dorniak et al., 2011; 2012a; 2012b; 2013b). 
The enzyme HSD11B1, which is involved in regulation of intracellular levels of bioactive glucocorticoids (GCs), has been suggested to be involved in elongation of the conceptus and to be regulated by $\mathrm{P}_{4}$ (Satterfield et al., 2009; Simmons et al., 2010). In the endometrium, $\mathrm{P}_{4}$ induces, and IFN- $\tau$ stimulates expression of $H S D 11 B 1$ and HSD11B1 activity to increase regeneration of cortisol from cortisone during early pregnancy in sheep (Satterfield et al., 2009; Simmons et al., 2010). Effects of $\mathrm{P}_{4}$ and IFN- $\tau$ are partly mediated by PGs based on a study reporting reduced HSD11B1 activity and cortisol levels in the uterine lumen of ewes given an intrauterine infusion of a PTGS inhibitor from days 8-14 of pregnancy, whereas, cyclic ewes receiving intrauterine infusions of IFN- $\tau$ or PGs had increased endometrial HSD11B1 expression and activity and increased uterine lumen cortisol levels (Dorniak et al., $2012 b$ ). Both the ovine uterine endometrium and the conceptus use the enzyme to turn cortisone into active cortisol, which then regulates the expression of genes involved in lipid metabolism and triglyceride homeostasis.

Cortisol acts through the GC receptor located in all endometrial cells of the ovine uterus during the estrous cycle and pregnancy and in trophectoderm (Dorniak et al., 2011; Satterfield et al., 2009; Simmons et al., 2010). When cortisol or IFN- $\tau$ were administered to cyclic ewes from 10-14 days post estrus by intrauterine infusion; HSD11B1 expression and activity was stimulated, there was increased PTGS2 activity and increased PG in the uterine lumen suggesting cortisol increases total PGs in the uterine lumen via effects on PTGS and HSD11B1 in the endometrium. In addition, intrauterine infusion of cortisol or INF- $\tau$ resulted in up-regulation of genes in the endometrium related to conceptus elongation (Dorniak et al., 2013b). Intrauterine infusion of a HSD11B1 inhibitor into cyclic ewes resulted in reduced expression of several $\mathrm{P}_{4}$ induced epithelial genes implicated in governing conceptus elongation and, indeed, the conceptuses failed to elongate when infused into pregnant ewes from day 10-14 post mating. However, IFN- $\tau$ infusion along with the enzyme inhibitor rescued elongation suggesting that HSD11B1 derived cortisol is involved in mediating ovarian $\mathrm{P}_{4}$ actions on endometrial function (Dorniak et al., 2013b). This set of studies suggest that HSD11B1 derived cortisol helps mediate actions of ovarian $\mathrm{P}_{4}$ and works with IFN- $\tau$ and PGs to regulate endometrial functions necessary for conceptus elongation and implantation (Simmons et al., 2010; Dorniak et al., 2012b; Dorniak et al., 2013b).

During the pre-implantation period, endometrial gene expression has been found to be significantly different compared to the equivalent day of the estrous cycle (Grey et al., 2006). Genes with differential expression included ones involved in the innate immune response and variations in endometrial gene expression coincided with changes in immune cell populations (Sandra et al., 2015). It is important that the uterine immune system is modulated to prevent the embryo from being rejected (Lea and Sandra, 2007; Hansen, 2010; Walker et al., 2010). The uterus is not an immunologically inert site; the 
immune system needs to be present for prevention of reproductive tract infections caused by the introduction of microorganisms during mating or parturition (Hansen, 2010). Progesterone has a major influence on the immune system preventing rejection of allografts and clearance of infections (Hansen, 2010).

\section{Immune System at the Feto-Maternal Interface}

The maternal-fetal interface once was thought to be immunologically inert but it is now understood that the uterus contains numerous types of immune cells playing important roles in establishing an adequate microenvironment during pregnancy, tolerance to "non-infectious self" (placenta, fetus), placental development, spiral artery remodeling, and elimination of 'infectious non-self' (pathogens) (Trowsdale and Betz, 2006; Koga et al., 2014). Subfertility can be due to disturbances in preimplantation embryonic growth and/or endometrial receptivity; both processes are heavily influenced by the maternal immune system. Development and acquisition of implantation competence by the blastocyst are influenced by the cytokine environment within the female reproductive tract while fetal tolerance and endometrium receptivity are affected by the phenotypes of the immune cell populations present at the implantation site (Robertson and Moldenhauer, 2014).

The maternal immune system recognizes fetal antigens at the decidual-trophoblast interface and peripherally due to shedding of fetal antigens into maternal circulation (Lissauer et al., 2009) leading to maternal immunological awareness of the developing fetus (Tafuri et al., 1995). In order to have a successful, uncomplicated pregnancy, the maternal immune system has to tolerate the semi-allogeneic fetus (Erlebacher, 2013; Sharma, 2014). Several mechanisms are involved in generating maternal-fetal tolerance including a shift towards T helper cytokine production of a "tolerogenic profile" (Saito et al., 2010), inhibition of complement activation (Mellor et al., 2001; Girardi et al., 2006b), chemokine gene silencing that prevents accumulation of activated T cells (Nancy et al., 2012) expansion of uterine immunomodulatory natural killer (uNK) cells (Tabiasco et al., 2006) and generation of regulatory T cells (Tregs) specific to paternal antigens (Rowe et al., 2012a). Typical immunoregulatory molecules in this environment include indoleamine 2,3-dioxygenase (IDO), tryptophan 2,3-dioxygenase (TDO), programmed death ligand 1 (PDL1-an inhibitory T cell costimulatory molecule), galectins, interleukin-10 (IL-10), and transforming growth factor-beta (TGF- $\beta$ ) (Curti et al., 2009; Blidner and Rabinovich, 2013; Santner-Nanan et al., 2013; Zhang et al., 2013).

IDO and TDO catabolize tryptophan, and the subsequent decrease can suppress T cell activity defending against maternal rejection (Munn et al., 1998; Suzuki et al., 2001). Tryptophan is an essential amino acid that is needed for cell proliferation, and $\mathrm{T}$ cells are sensitive to changes in local concentrations of the amino acid (Trowsdale and Betz, 2006). IDO is produced by some tissue MФ and antigen 
presenting cells (APCs) in response to interferon gamma (IFN $\gamma$ ), by fetal syncytiotrophoblasts, and by the decidua during pregnancy. It can control $\mathrm{T}$ cell-dependent inflammatory responses to fetal alloantigens by regulating T cell proliferation and activity (Munn et al., 1998; Caucheteux et al., 2003; Trowsdale and Betz, 2006). Galectins (Gal) are a family of $\beta$-galactoside-binding lectins expressed by several types of immune cells and by tissue resident stromal cells that have functions related to the activation (or prevention of), proliferation, survival., and migration of effector T cells along with influencing what cytokines are produced by T cells. Specifically, gal-1 is important for fetal survival as it functions in the generation of tolerogenic dendritic cells (tDCs) and Tregs, can induce the death of effector T cells, stimulates the production of anti- verses pro- inflammatory cytokines (PICs) by T helper (Th) cells, and controls transendothelial T cell migration (Chung et al., 2000; Stillman et al., 2006; Blois et al., 2007b; Rabinovich et al., 2007; Toscano et al., 2007; Motran et al., 2008; Norling et al., 2008; Ilarregui et al., 2009; Blidner and Rabinovich, 2013).

The Th1/Th2 paradigm was developed to describe a predominantly Th2 cytokine profile during pregnancy to prevent fetal rejection (Wegmann et al., 1993). Indeed, Th2 cytokines have been shown to be necessary to prevent fetal resorption, whereas increased levels of Th1 cytokines cause fetal loss (Chaouat et al., 1995). However, with continued research this paradigm seems to be an oversimplification of what is occurring at the feto-maternal interface. Instead, there seems to be sequential windows of a series of cytokine interactions that govern immune regulation, adhesion, vascularization, and implantation in the uterus (Chaouat et al., 2002). For example, the process of implantation has been found to rely on several PICs (ex: LIF) that regulate expression of adhesion molecules and their receptors, like laminin and fibronectin, on trophoblasts and uterine decidual cells (Stewart et al., 1992; Chaouat et al., 2002; Dimitriadis et al., 2005).

Even though PICs are important signaling molecules at the beginning of pregnancy, immune mediators and the implantation process must be tightly regulated to obtain the correct amount of blastocyst invasion and angiogenesis. What has become to be appreciated is that the immune mediators at the feto-maternal interface are not strictly pro- or anti- inflammatory, but both types of cytokines have specific roles, and they must be produced in appropriate quantities in a correct temporal manner for pregnancy success. For example, uNKs regulate trophoblast invasion and uterine vascular remodeling by secreting angiogenic factors and chemokines for cells involved in the vascular and tissue remolding during implantation including CXCL8, IP-10, (interferon-inducible protein-10) and IFN $\gamma$ (Ashkar et al., 2000; Hanna et al., 2006). However, as a PIC, IFN $\gamma$ can cause fetal loss when produced in large quantities in response to infection or in some cases the presence of foreign paternal antigen (Chaouat et al., 1995). After implantation, the uterine environment is dominated by anti-inflammatory factors until parturition. 
Early pregnancy involves implantation, placentation, and vascular remolding; events that include invading cells, cells that are dying, and cells that are repairing tissue. A controlled inflammatory environment in the uterus is needed to have adequate repair of the uterine epithelium and removal of cellular debris. Early pregnancy has been characterized by the presence of CXCL8, Macrophage Chemoattractant Protein 1 (MCP-1), RANTES (Regulates on activated normal T cells where it is expressed and secreted), and Granulocyte-Colony Stimulating Factor (G-CSF) (Mor, 2008). One source of these chemokines are monocytes that are induced to produce them in response to trophoblast cells (Fest et al., 2007). While these factors tend to be immune stimulatory, RANTES also has immunosuppressive functions (Ramhorst et al., 2004). Trophoblast cells themselves have been found to produce chemokines such as GRO- $\alpha$, MCP-1, and CXCL8 that attract monocytes and macrophages (Abrahams et al., 2005; Fest et al., 2007). These cells are important APCs and are involved in tissue remodeling and spiral artery transformation (Abrahams et al., 2004). The monocytes will evolve into MФs and surround the trophoblast. In the study by Fest et al (2007) Macrophages stimulated trophoblast cells to secrete more IL6 and MCP-1, indicating that monocytes recruited by the trophoblast may then support implantation by communicating with trophoblast cells and providing appropriate signals for implantation. After placentation, the uterine environment is predominantly anti-inflammatory until the end of gestation as parturition is also an inflammatory event (Mor, 2008). While some inflammation is needed to get adequate implantation, tipping the scales too far towards pro-inflammation or inflammation as a result of infection or failure to tolerate paternal antigens can cause embryonic loss.

The process of implantation involves extensive tissue remolding and apoptosis of cells that have to be cleared to prevent the release of antigens (Abrahams et al., 2004). Macrophages at the feto-maternal interface have the job of scavenging the dying cells as well as actively orchestrating apoptosis of unwanted cells during tissue remodeling (Savill and Fadok, 2000). In response to phagocytosing apoptotic cells, MФs synthesize and secrete cytokines and growth factors that govern local cellular and tissue interactions (Hunt, 1989; Garcia-Velasco et al., 1998). Specifically, uptake of apoptotic cells induces $M \Phi_{\text {s }}$ to produce anti-inflammatory/immunosuppressive cytokines (IL-4, IL-6, IL-10) while suppressing MФs from secreting PICs (TNF- $\alpha$, IFN $\gamma$ ) (Abrahams et al., 2004). These uterine MФs have an M2 phenotype characterized not only by the production of immune-inhibitory cytokines (TGF $\beta$, IL-10) (Heikkinen et al., 2003) but also by the production of pro-tolerance factors $\mathrm{PGE}_{2}$ (Tawfik et al., 1986) and IDO (Munn et al., 1999; Renaud and Graham, 2008), upregulation of arginase activity (counteracts NO synthesis) (Munder et al., 1998), and increased secretion of the IL-1 receptor antagonist (IL-1Ra) (Fenton et al., 1998). Uterine MФs also produce and regulate matrix metalloproteinases (MMPs), proteases, growth factors, chemokines, and cytokines that influence implantation and placental development 
(Renaud and Graham, 2008). However, a uterine infection can induce MФs to produce IL-12, IL-1 and TNF $\alpha$ through activation of PPRs, or through phagocytosing an overabundance of apoptotic or necrotic cells (Hunt, 1989; Abrahams et al., 2004; Nagamatsu and Schust, 2010). Thus, pathogen exposure can shift polarity of uterine MФs from an M2 to an M1 inflammatory phenotype (Nagamatsu and Schust, 2010).

Trophoblast cells stimulate MФs to produce inflammatory mediators needed for implantation but may also be able to limit their inflammatory potential during a minimal threat. M $\Phi$ s exposed to trophoblast cells followed by stimulation with a low dose of LPS $(0.1 \mu \mathrm{g})$ did not have an increase in inflammatory mediators compared to MФs only exposed to LPS (Fest et al., 2007). However, when a high dose of LPS was used $(10 \mu \mathrm{g})$ the trophoblast cells were unable to control the MФ's inflammatory response (Fest et al., 2007). High doses of LPS mimic bacterial overgrowth and infection that have detrimental effects on pregnancy (Deb et al., 2004). Abortion can result with high doses of LPS (Murphy et al., 2005) as a result of the release of embryotoxic substances and excessive release of PICs (IL-1, IL-6, TNF $\alpha$ ) from MФs (Hill et al., 1987; Hunt, 1990). Therefore, there is a tipping point where the infection is severe enough that the immune system "chooses" to use all its force in clearing the infection to protect the mother over continuing the pregnancy.

One critical immune cell type during pregnancy is maternal Tregs. While Tregs are specific to paternal alloantigen, expansion of the Treg population is not dependent on exposure to paternal alloantigen as the population expands in both allogeneic and syngeneic pregnancies (Tafuri et al., 1995; Aluvihare et al., 2004), and suppression by Tregs is not antigen specific (Chaouat et al., 2012). In addition, maternal $\mathrm{T}$ cells acquire only a transient state of tolerance for those paternal alloantigens (Tafuri et al., 1995), which has been demonstrated in vivo with graft rejection studies in female mice. Pregnant mice accept an allogeneic tumor graft as long as the paternal graft displayed the same MHC-peptide complexes as the fetus; a graft from a father with different MHC peptides was rejected. In addition, the mother would tolerate the allogenic tumor graft only while she was pregnant; after parturition the tumors were rejected (Tafuri et al., 1995). In order to generate immune tolerance to paternal antigens the antigens need to be presented to maternal immune cells. Generation of tolerance starts before pregnancy with antigens present in seminal plasma deposited during copulation (Roberson et al., 2013) being presented to $\mathrm{T}$ cells. Continued suppression of maternal immune responses to paternal alloantigens may involve maternal APCs picking up fetal antigen from buds and microparticles that are shed from the trophoblast into the maternal circulation (Chamley et al., 2011). Fetal tissue remodeling involving apoptosis that generates cellular debris also is a likely source of fetal antigens that can be presented, as phagocytic cells like APCs would be responsible for cleaning up the dead cells. 
Abumaree et al (2012) showed that phagocytosis of trophoblast debris in vitro by MФs resulted in protein expression changes in the MФs that would encourage maternal tolerance to be generated. MФs that phagocytosed trophoblast debris reduced cell-surface expression of MHC-II molecules, costimulatory molecules (CD80, CD86, CD40), MCP-1, and ICAM-1 while upregulating PD-1 expression. MФ expression of IDO was increased after exposure to trophoblast debris. These MФs increased secretion of IL-10, IL-6, and IL-1Ra, while decreasing secretion of IL-1 $\beta$, IL-12p70, and CXCL8 (Abumaree et al., 2012). Downregulating MHC-II and co-stimulatory molecules means the MФ is less able to activate effector $\mathrm{T}$ cells and, with increased IDO, is able to regulate $\mathrm{T}$ cell proliferation. These changes will make the $\mathrm{MФ}$ guide maternal immune responses toward tolerance of the fetal antigens instead of an inflammatory response.

The importance of downregulating co-stimulatory molecules was shown in murine studies where blocking CD86 or CD80 and CD86 with antibodies early in gestation shifted the cytokine profile to a Th2 dominance at the feto-maternal interface, and caused expansion of peripheral Treg cells, which led to decreased fetal resorption in abortion prone mice pairings (Jin et al., 2005; Zhu et al., 2005).

The PD-1/PD-L1 pathway has been reported to be critical for feto-maternal tolerance, blocking PD-1 signaling increases rejection of allogenic but not syngeneic fetuses in mice (Guleria et al., 2005; Wafula et al., 2009; Zhang et al., 2015). PD-1 is a receptor involved in co-stimulatory interactions between immune cells. Co-stimulatory signals are the second signal provided to T cells being shown antigen. The first signal is interaction between the T cell receptor (TCR) and the MHC molecule with bound antigen. Co-stimulatory molecules are either positive secondary signals that promote T-cell activation or negative signals that inhibit T-cell responses and mediate T-cell tolerance. PD-1 delivers inhibitory signals and its expression can be induced on CD4+ T cells, CD8+ T cells, NK T cells, B cells, and by activated monocytes (Sharpe et al., 2007). The PD-1 receptor responds to two ligands: PD-L2, which is expressed by DCs and MФs and PD-L1, which is constitutively expressed by B cells, DCs, MФs, and T cells (Sharpe et al., 2007). PD-L1 also can be expressed by non-hemopoietic cells in certain locations, including the placenta (Zhang et al., 2015). PD-1 is involved in inhibiting the activation of effector T cells while promoting development and function of induced regulatory $\mathrm{T}$ cells (Francisco et al., 2009; Zhang et al., 2015) Tregs express high levels of both PD-1 and PD-L1 (Lohr et al., 2006), and PDL1 has been shown to increase the generation of Treg cells from naïve CD4+ T cells in the presence of TGF $\beta$, increase expression of FOXP3, and is produced by Tregs to suppress activated effector T cells (Francisco et al., 2009; Zhang et al., 2015). 


\section{Uterine Natural Killer Cells}

The predominant leukocyte population in the endometrium during implantation and early pregnancy are natural killer cells (NK cells) (Croy et al., 2006). NK cells may migrate from the peripheral blood into the endometrium in response to chemokines upregulated in response to steroid hormones (Sentman et al., 2004; Carlino et al., 2008; Cerdeira et al., 2013) or may be derived from CD34+ cell precursors that differentiate into uNKs after interaction with decidual cells (Vacca et al., 2011b). Trophoblast cells also secrete chemokines to actively recruit NK cells to the area (Hanna et al., 2003).

The NK cells are important players in immune responses to infections, and peripheral NK cells fight pathogen infections through their cytotoxic activity of perforin and granzyme secretion. These cells can have two major phenotypes; however, $\mathrm{CD} 16^{+} \mathrm{CD} 56^{\mathrm{dim}} \mathrm{NK}$ cells have high cytotoxic potential but are not major producers of cytokines whereas $\mathrm{CD} 16^{\text {neg }} \mathrm{CD} 56^{\text {bright }} \mathrm{NK}$ cells have poor cytotoxic activity (Crncic et al., 2007). The majority ( 90\%) of peripheral NK cells are highly cytotoxic with 10\% producing high levels of cytokines (Koopman et al., 2003). The uNK (CD16 ${ }^{\text {neg }}$ CD56 $6^{\text {bright }}$ ), however, are specialized in that even though they possess the machinery (perforin and granzyme cytotoxic granules) to lyse cells, they have low cytotoxic activity (Koopman et al., 2003; Kopcow et al., 2005; Crncic et al., 2007), which may be regulated by $\mathrm{P}_{4}$ upregulating gene expression of non-polymorphic MHC molecules, which can bind to inhibitory receptors on NK cells (Yie et al., 2006).

Instead of having a cytotoxic role in the uterus, uNKs play an important role in establishing and maintaining maternal-fetal immune tolerance, in inducing Treg cells, acting as local guardians against infection, controlling trophoblast invasion and vascular angiogenesis through the secretion of angiogenesis-regulating molecules (Hanna et al., 2006; Tabiasco et al., 2006; Kalkunte et al., 2009; Vacca et al., 2010; Vacca et al., 2011a; Winger and Reed, 2013). The uNK cells, like other immune cells at the feto-maternal interface, express high levels of gal-1, which is a critical mediator of immune tolerance during pregnancy (Koopman et al., 2003; Blois et al., 2007b; Rabinovich and Toscano, 2009). The trophoblast and maternal-fetal interface is assumed to modify recruited peripheral NK cells, changing the phenotype and function to that of uNK cells (Du et al., 2014; Tao et al; 2015; Li et al., 2016).

Uterine NKs produce IFN $\gamma$ early in pregnancy that acts in an autocrine manner to further stimulate IFN $\gamma$ production in addition to vascular endothelial growth factor (VEGF), angiopoietin 2, and PGF involved in angiogenesis in early pregnancy and nitric oxide (NO), which stimulates vasodilation (Warning et al., 2011). Interferon- $\gamma$ (IFN- $\gamma$ ) also has been shown to regulate inflammatory Th17 cells (Fu et al., 2013). While IFNץ contributes to the important placentation step of spiral artery remodeling (Ashkar et al., 2000), production of the cytokine must be regulated as elevated levels, like during an infection, can cause fetal loss (Shiono et al., 2007). 
While uNKs do not display the cytotoxic functions of their peripheral counterparts, uncontrolled or exaggerated inflammation at the maternal-fetal interface can cause uNKs to revert back into their peripheral NK phenotype and cause embryonic loss (Murphy et al., 2005; Murphy et al., 2009; KwakKim et al., 2014). IL-12 stimulates IFN $\gamma$ production by NK cells and induces their cytotoxic activity. The PICs TNF $\alpha$, IL-1 $\beta$, and IL-15 synergize with IL-12 to induce IFN $\gamma$ production and IL-15 also promotes NK cell maturation. IL-10 can regulate IFN $\gamma$ production by NK cells by inhibiting IL-12 production while TGF $\beta$ can block proliferation and cytotoxicity of NK cells as well as inhibit IFNץ and IL-12 production (Biron et al., 1999; Eriksson et al., 2004; Eriksson et al., 2006). IL-10 and TGF $\beta$ are produced by Tregs and tDCs in the uterus to regulate fetal tolerance and the amount of the cytokines produced at the fetomaternal interface (Simpson et al., 2002) regulating the response/phenotype of uNK to pathogens.

When uNK cells gain cytotoxic capability during inflammation they cause apoptosis of trophoblast cells with the transfer of granulysin with the help of perforin (Nakashima et al., 2008). Several authors haves suggested that LPS can act on NK cells directly and that uNK cells are major players in LPS stimulated embryonic loss. The NK cells express TLR4 (Duriez et al., 2014), and treatment of uNK cells results in production of TNF- $\alpha$ (Li et al., 2016). LPS has been shown to recruit additional NKs to the uterus and trigger a phenotypic change in uNK cells that leads to abortion. This effect is likely to be mediated by PICs as depletion of NK cells or neutralization of TNF $\alpha$ prevented pregnancy loss (Murphy et al., 2009). LPS not only triggers a robust inflammatory response characterized by the production of PICs, such as TNF- $\alpha$ and IL- $1 \beta$, and a shift in the Th1/Th2 balance at the fetalmaternal interface (Park et al., 2009) but also has been reported to induce uNK cytotoxic activation that could lead to NK cell mediated killing of embryonic cells (Murphy et al., 2005). Indeed, treatment of peripheral blood mononuclear cells with LPS results in proliferation and enhanced cytotoxic activation of NK cells (Miranda et al., 1998; Goodier and Londei, 2000). In IL-10 null mice treated with LPS, depletion of NK cells can restore pregnancy (Murphy et al., 2005). IL-10 null mice are missing a cytokine used to regulate the phenotype of NKs in the uterus making it easier for them to gain cytotoxic capability. Restoration of pregnancy in an LPS model by depleting these cells demonstrates how critical it is that they maintain their phenotype.

\section{Regulatory T Cells}

Regulatory T cells are a subset of CD4+ T cells that express FOXP3 and CD25 constitutively and can suppress immune responses in an antigen-independent fashion (Sakaguchi, 2004; Wan and Flavell, 2006). There are two ways for Tregs to be generated. Natural Tregs (nTregs) originate in the thymus by a selective process involving $\mathrm{T}$ cells being presented with self-antigens and gaining a regulatory function. Inducible Tregs are produced in lymph nodes by a mechanism that generates Tregs with receptors specific 
for antigens not expressed in the thymus but instead restricted to different peripheral tissues (Shevach et al., 2002). The APCs can induce naïve CD4+ cells to become Tregs (iTregs) by presenting antigen that is recognized by their T cell receptor while producing IL-2 and TGF 3 (IL-10 may also contribute) which leads to FOXP3 expression (Chen et al., 2003; Horwitz et al., 2003; Fantini et al., 2004; Wan and Flavell, 2006; Murphy, 2012).

Treg cells are characterized by the transcription factor, FOXP3. In order for a naïve CD4+ T cell to polarize into a Treg cell, TGF $\beta$ must be present at the time of TCR ligation with its antigen. The APC not only provides the antigen to stimulate the naïve $\mathrm{CD} 4+\mathrm{T}$ cell but produces the cytokines that determine how the naïve CD4+ cell is polarized in response to that particular antigen (Chen et al., 2003). TGF $\beta$ is important during Treg polarization because it induces FOXP3 expression via SMAD3- and retinoic acid receptor binding sites (Zheng et al., 2010). TGF $\beta$ also may aid in the proliferation of mature Tregs by modifying the function and signaling capabilities of DCs (Ghiringhelli et al., 2005b). PGE 2 , also delivered to the female reproductive tract during mating, may work with TGF $\beta$ in generating Tregs as $\mathrm{PGE}_{2}$ has been reported to enhance the inhibitory capacity of human Treg cells in vitro and to induce a regulatory phenotype in CD4+CD25- T cells (Baratelli et al., 2005).

After the naïve CD4+ T cell has recognized its antigen, it is activated, will proliferate and then leave the lymph node (Walker et al., 2003). Inducible Tregs are critical for successful pregnancy because Tregs with TCRs specific to paternal alloantigens, which are foreign to maternal self, must be generated to control aggressive immune responses to foreign looking antigens displayed by the conceptus (Samstein et al., 2012). While all inducible Tregs generated in peripheral tissues have to undergo antigen driven activation and proliferation to gain full suppressive function, actual suppressive effector function is antigen non-specific (Thornton and Shevach, 1998; Samy et al., 2006). The maternal immune system first encounters paternal antigens when seminal fluid is delivered to the female reproductive tract at coitus (Robertson and Sharkey, 2001). Treg cells populations start to expand within days of mating (Aluvihare et al., 2004) and must be at the feto-maternal interface and functional before implantation (Zenclussen et al., 2005; Shima et al., 2010).

$\mathrm{CD} 25$ is the alpha chain of the IL-2 receptor; upon IL-2 binding, the IL-2R $\alpha, \beta$, and $\gamma_{\mathrm{c}}$ chains oligomerize, and a JAK (Janus Kinase)-STAT (signal transducer and activator of transcription) pathway is activated. IL-2 is required for the generation and maintenance of Tregs (Horwitz et al., 2003). JAK1 is associated with IL2R $\beta$ while JAK3 is associated with IL-2R $\gamma_{c}$ but can also interact with the $\beta$ chain. The activated JAKs phosphorylate tyrosine residues in the $\beta$ chain then phosphorylate STAT5, which is 
responsible for expression of FOXP3, the transcription factor for development of Tregs and their immunosuppressive phenotype (Wan and Flavell, 2006).

At ovulation, the elevated $\mathrm{E}_{2}$ levels cause a systemic expansion in Tregs and recruitment to the uterus by inducing FOXP3 mRNA expression and increasing uterine expression of Treg chemokines, CCL3, CCL4, and CCL5 (Polanczyk et al., 2004; Kallikourdis and Betz, 2007). The corpus luteum (CL) is formed after ovulation, and $\mathrm{P}_{4}$ may be involved in sustaining and further expanding Treg populations. In vitro, $\mathrm{P}_{4}$ was able to convert CD4+CD25- $\mathrm{T}$ cells into CD4+CD25+ Treg cells (Mao et al., 2010). $\mathrm{P}_{4}$ was also seen to be able to convert naïve fetal (cord blood) $\mathrm{T}$ cells into Treg cells that had been allogenically activated. These differentiated Treg cells expressed FOXP3, high levels of memory T-cell markers and had impaired expression of the IL-6 receptor, which should stabilize them towards having a regulatory phenotype and inhibit conversion to a Th17 cell (Lee et al., 2011). The $\mathrm{E}_{2}$ induced Treg expansion occurs after every ovulation with $\mathrm{E}_{2}$ probably targeting Tregs regardless of their TCR specificity meaning these Tregs will not be specific to paternal alloantigen. Indeed, $\mathrm{E}_{2}$ was seen to expand Treg cells in different tissues in mice while in vitro $\mathrm{E}_{2}$ converted CD4+CD25- cells, to CD4+CD25+ cells which then expressed FOXP3 and IL-10 and were able to suppress naïve T cell proliferation in a mixed lymphocyte reaction (Tai et al., 2008).

To be activated and have suppressive function, inducible Tregs require TCR ligation with antigen and appropriate polarizing signals. During coitus, seminal fluid delivers paternal antigens that also will be expressed by conceptus tissues as well as TGF $\beta$ and $\mathrm{PGE}_{2}$ into the female reproductive tract (Robertson et al., 2009; Robertson et al., 2013; Schjenken and Robertson, 2014). This will cause DCs to be recruited to the uterus, mature into a tolerogenic phenotype, pick up the alloantigen and take it to the draining lymph nodes to present to naïve CD4+ T cells polarizing them towards a Treg phenotype by TCR ligation with the antigen in the presence of IL-2 and TGF $\beta$. These Treg cells will be specific to alloantigen and are recruited to the uterus by chemokine CCL19 that is expressed on glandular and luminal epithelial cells and binds to the CCR7 receptor on Tregs (Guerin et al., 2011). Seminal fluid is responsible for induction of uterine expression of CCL19 mRNA, which regulates Treg cell retention in peripheral tissues (Alt et al., 2002; Engelhardt et al., 2006). The presence of sperm in addition to seminal fluid was reported to be required for maximal expression of the chemokine and local Treg accumulation in the uterus before implantation (Guerin et al., 2011). Interaction with paternal antigens also induces Tregs to express the chemokine receptor CCR5, and the chemokine CCL4 is involved in sequestering activated, antigenspecific CCR5+ Treg cells to the gravid uterus (Kallikourdis et al., 2007). During and after implantation, tissue remodeling of gestational tissues and the growing fetus will release additional alloantigens and the chemokine CCL4 to further expand and recruit Treg cell populations to the feto-maternal interface 
(Robertson et al., 2013). Placental trophoblasts secrete TGF $\beta$ that is likely involved in regulating the Treg population. Trophoblast cells have been shown to induce FOXP3 expression along with a Treg cytokine profile in naïve T cells and to secrete inducible Treg chemoattractants (Ramhorst et al., 2012).

With regard to the $\mathrm{E}_{2}$ induced peri-ovulatory expansion of the Treg cell pool, seminal fluid provides the signals to select clones that are specific to paternal antigens from the broader TCR repertoire and expand them to provide protection for the fetus (Robertson et al., 2013). TGF $\beta$, derived from male accessory glands like the prostate and seminal vesicle (Robertson et al., 2002), along with PGE-related prostaglandins have been associated with the generation of Treg cells by inducing naïve CD4+CD25- T cells to differentiate into suppressor T cells that express FOXP3 (Chen et al., 2003; Baratelli et al., 2005). Studies have shown that seminal fluid and TGF $\beta$ in the seminal fluid delivered during coitus are responsible for activation and proliferation of Tregs. In mice, Treg cells are increased about 2 fold 3.5 days post-coitus compared with the estrus associated increase in cycling females, but if seminal vesicles were removed from males before mating, the increase did not occur (Robertson et al., 2009; Guerin et al., 2011). The importance of TGF $\beta$ is shown by studies in which exogenous TGF $\beta$ given to female mice of abortion-prone matings increased vaginal Treg cell numbers and reduced fetal loss (Clark et al., 2008).

After becoming activated and fully functional, Tregs leave the lymph nodes and travel through the circulation to the appropriate peripheral location in response to chemokines where they interact with other cells in the local environment to exert immune suppressive functions in an antigen non-specific manner (Thornton et al., 2000). Immunosuppressive functions of Tregs are mediated by cell-cell contact involving cell-surface molecules, by the production of specific cytokines, and by competitive deprivation of other T cells of IL-2 (Thornton et al., 1998; Nakamura et al., 2001; Boussiotis et al., 2001; Scheffold et al., 2005; Wan and Flavell, 2006; Shevach, 2009). Tregs suppress the proliferation of other T cell subtypes, the production of IFN $\gamma$ and IL-2 by effector cells and can suppress immunoglobulin production by B cells (Thornton and Shevach, 1998; Nakamura et al., 2001; Garín et al., 2007). Mature Tregs secrete the cytokines IL-10 and TGF $\beta$ which further expand the Treg cell response through paracrine suppressive properties (Fontenot et al., 2003; Hori et al., 2003; Wahl et al., 2004).

Besides CD25, other surface markers on Tregs include cytotoxic T-lymphocyte antigen-4 (CTLA-4), glucocorticoid-induced tumor necrosis factor receptor (GITR) family-related gene, lymphocyte activation antigen-3 (LAG-3) (Sakaguchi, 2004; Huang et al., 2004) and the cell surface bound form of TGF (Nakamura et al., 2001). These surface molecules are used for cell contact mediated actions of Tregs (Thornton et al., 2000). TGF $\beta$ also is secreted when CD3 is stimulated on the Treg, and secretion is enhanced when CD28, IL-2R, and CTLA4 are engaged in co-stimulation (Nakamura et al., 
2001). LAG3 is a CD4-related molecule that binds MHC class II molecules on APCs, and interaction between Tregs and immature DCs using these molecules induces an ITAM (immunoreceptor tyrosinebased activation motif) mediated inhibitory signaling pathway in the DC that prevents maturation and immunostimulatory capacity of the APC (Huang et al., 2004; Liang et al., 2008).

Tregs constitutively express CTLA4 (Takahashi et al., 2000) and ligation of the molecule induces production of TGFß (Chen et al., 1998) by the Treg cell, which enhances their suppressive function. CTLA-4 interacts with CD80/CD86 on APCs, which can prevent the up-regulation (or cause downregulation) of expression of these co-stimulatory molecules by APCs (Oderup et al., 2006; Onishi et al., 2008). Reduced expression of CD80 and CD86 by APCs will limit the ability of the cells to activate naïve $\mathrm{T}$ cells through CD28 and in fact, antigen presentation without sufficient co-stimulatory molecule interaction can cause a naive $\mathrm{T}$ cell to become anergic (Murphy, 2012). Crosslinking of CTLA-4 with CD3 and CD28 inhibits T cell proliferation, IL-2 secretion, suppression of IFN $\gamma$ from Th1 cells and IL-4 from Th2 cells (Chen et al., 1998).

Tregs, through CTLA4-CD80/CD86 interaction along with production of TGF $\beta$ and IL-10 induce local DCs to express IDO, which catabolizes tryptophan (Grohmann et al., 2002; Fallarino et al., 2003). Expression of IDO by DCs also is a mechanism for DCs to activate Tregs and stimulate them to maintain suppressive functions. Thus, a positive feedback loop to ensure a suppressive local immune environment is created (Sharma et al., 2007, Grohmann et al., 2002).

Tregs influence the population of T helper cells in the local environment by inhibiting Th1 cell proliferation and inducing Th1 cell apoptosis (Robertson et al., 2013). IDO catabolizes tryptophan to kynurenine, which is toxic to T cells that are neighboring the producing cell (La Rocca et al., 2014). Tryptophan metabolites can inhibit activation of T cells and NK cells (Frumento et al., 2002, Terness et al., 2002), and low doses of kynurenine induce selective apoptosis of Th1 versus Th2 cells (Terness et al., 2002; Fallarino et al., 2002), which could aid in creating a Th2 cytokine bias in the uterus. Tregs also can kill cells in a granzyme-and perforin dependent manner or send negative signals to the targeted cell that control its function, proliferation, and survival (La Rocca et al., 2014). Tregs provide signals to responding T cells through the co-stimulatory molecules CD80 and CD86 expressed on the responder cell (Paust et al., 2004). In a cell-contact mediated mechanism, cAMP is transferred through gap junctions from Treg cells to the responder T cells where it inhibits proliferation and IL-2 production (Bopp et al., 2007). In a different mechanism, using surface expressed CD39 and CD37, the Treg cell generates pericellular adenosine from extracellular nucleotides that binds to the adenosine A2A receptor on activated effector T cells (Deaglioet al., 2007) inhibiting T cell activation, proliferation, and expansion (Huang et al., 1997). Extracellular ATP is a DAMP signal that can increase CD86 expression on DCs; 
activated Tregs express CD39, which hydrolyzes extracellular ATP to AMP thus eliminating the inflammatory signal (Shevach, 2009). Tregs express Nrp-1, which promotes long interactions between the Treg cell and immature DCs and in doing so limits interactions between effector cells and the APC (Shevach, 2009).

The importance of Tregs during pregnancy is demonstrated in murine studies showing Treg deficient BALB/c nude females transferred Treg depleted polyclonal $\mathrm{T}$ cells experienced fetal resorption in allogenic pregnancies but not in syngeneic pregnancies (Aluvihare et al., 2004). Regulatory $\mathrm{T}$ cell depletion with anti-CD25 monoclonal antibodies during implantation resulted in implantation failure or during early pregnancy resulted in fetal resorption in allogeneic but not syngeneic pregnancies (Munn et al., 1998; Darrasse-Jéze et al., 2006; Shima et al., 2010). The Tregs that expand during pregnancy are specific to fetal antigens (Kahn and Baltimore, 2010; Rowe et al., 2012a). Comparing synergistic and allogenic mice matings, allogeneically pregnant females had a greater increase in Treg number, which resulted in enhanced immune suppression to paternal antigens. (Kallilourdis et al., 2007; Zhao et al., 2007). Decidual lymphocytes from mice that had abortions produced significantly less IL-10 and significantly more TNFa than decidual cells from normal pregnant mice (Zenclussen et al., 2005). This group also reported that mice that had abortions had reduced numbers of Treg cells and showed that exposure to paternal antigen is necessary to generate Tregs that are competent at promoting protection to the fetus as transfer of Tregs from normal non-pregnant mice to pregnant abortion prone mice had no benefits. It is also critical that Tregs be at the feto-maternal interface during the peri-implantation period when the trophoblast starts making contact with maternal tissues, as transfer of Tregs after implantation in mice did not prevent abortion (Zenclussen et al., 2005). These results show that Tregs are important for generating maternal tolerance to paternal antigen expressed by the fetus, Tregs are important for successful implantation, and that Tregs must recognize fetal antigens to create an immunosuppressive environment.

\section{Seminal Fluid}

The affects of seminal fluid on the female reproductive tract have been well studied in mice. A response that resembles an inflammatory event occurs with the production of cytokines such as LIF, IL-6, and granulocyte colony stimulating factor (GM-CSF) from $\mathrm{E}_{2}$ primed epithelial cells that recruit additional innate and adaptive immune cells (Robertson et al., 1998; Tremellen et al., 1998). Within hours of mating, the numbers of MФ, DCs, neutrophils, T cells and granulocytes within the uterine endometrial stroma and lumen are increased (McMaster et al., 1992; Robertson et al., 1998; Tremellen et al., 1998). This transient inflammatory response to seminal fluid recruits additional immune cells to the female reproductive tract and is seen in other species including sheep (Scott et al., 2006). 
When the female is receptive to mating around ovulation, DCs are abundant in the endometrial tissue (Robertson et al., 1996; Blois et al., 2007a). The addition of seminal fluid at coitus further recruits APCs to endometrial tissues (Robertson et al., 1996) as a result of seminal fluid inducing expression of the chemokines CCL2, CCL3, CCL5, CCL20, CCL22, and CXCL10 (Robertson et al., 1998). The DCs in the endometrial tissue normally have a tolerogenic phenotype (Blois et al., 2007a), which will be maintained as long as cytokines such as GM-CSF, IL-4, and IL-10 are produced by uterine tissue and dominate the local cytokine environment (Robertson et al., 2000). The importance of seminal fluid is shown by in vitro studies in which seminal fluid is able to direct the differentiation of DCs into a tolerogenic phenotype that then produce higher amounts of IL-10 and TGF $\beta$, and can induce Treg differentiation (Remes et al., 2012). Within uterine tissues, tDCs will phagocytose spermatozoa, soluble antigens and apoptotic somatic cells in the seminal fluid deposited during coitus. Seminal fluid induces production of PICs in the female reproductive tract, which causes the DCs to traffic to the draining lymph nodes after uptake and processing of antigen or to interact with local Treg cells in the uterus. Both of these actions by DCs lead to the activation and expansion of clonal subsets of Treg cells that express TCRs that are specific to seminal fluid antigens (Moldenhauer et al., 2009a; Robertson et al., 2013). The $\mathrm{P}_{4}$ from the CL stabilizes the Treg cell pool, and, if conception is successful after mating, apoptotic placental and fetal cells from tissue remodeling provide further alloantigens to be cross-presented to $\mathrm{T}$ cells by maternal DCs, increasing the number of Treg cell clones specific to paternal alloantigens (Robertson et al., 2013). While seminal fluid and the conceptus share antigens, it is unlikely that all paternal antigens that will be expressed by the conceptus are presented to Tregs during initial Treg cell priming. Therefore, the notion of "bystander tolerance" mediated by Treg cells (Shevach et al., 2002; Corthay, 2009; Shevach, 2009; Kahn and Baltimore, 2010) is important during pregnancy meaning that a selection of paternal antigens that will be expressed by the conceptus are presented and used to generate Treg cell clones that then proliferate and generate an overall immune suppressive environment at the fetomaternal interface. Because Tregs are suppressive in an antigen independent manner once activated, so they are able to regulate the uterine immune environment without having been presented every paternal antigen that will be expressed during gestation.

Seminal fluid also may affect steroidogenesis. In mice, exposure to seminal fluid is associated with increased numbers of MФs in the CL (Gangnuss et al., 2004), and these innate immune cells seem to play a critical role in remodeling events required to sustain steroidogenic function (Care et al., 2013). Administration of seminal fluid to pigs at ovulation also has been associated with an increase in CL MФs along with increased steroidogenic function and increased circulating plasma $\mathrm{P}_{4}$ (O'Leary et al., 2006). 
Not only is $\mathrm{P}_{4}$ critical for maintenance of pregnancy and embryo development, this hormone has direct positive effects on Treg cell differentiation, proliferation and suppressive function (Mao et al., 2010).

\section{Transforming growth Factor Beta}

After being deposited into the female tract as a component of seminal fluid, TGF $\beta$ is activated by acidic vaginal $\mathrm{pH}$, enzymes originating in the male or female tract, or through conformational changes induced by interaction with epithelial cell docking proteins (Robertson et al., 2002). This cytokine has a role stimulating the post-coital inflammatory response, inducing maternal immune tolerance to seminal antigens, preventing immunity to sperm, and priming an appropriate maternal immune response to embryo implantation because many seminal antigens will be expressed by the conceptus (Robertson et al., 2002). Interacting with uterine and/or cervical epithelial cells, TGF $\beta$ upregulates several PICs and chemokines leading to recruitment and activation of leukocytes including neutrophils, MФs, and DCs (McMaster et al., 1992; Robertson et al., 1992a; Robertson et al., 1996) Also a component in seminal fluid, PGE aids in recruitment of leukocytes through promotion of CXCL8 expression (Denison et al., 1991). TGF- $\beta$ is involved in the induction and maintenance of Treg function (Clark et al., 1997;

Gabrysová et al., 2011). Tregs are generated in the lymph nodes beginning at mating and then circulate in the blood to be sequestered into the endometrium in a chemokine-dependent manner prior to implantation (Zenclussen et al., 2005; Robertson et al., 2009; Guerin et al., 2011; Shima et al., 2012; Robertson et al., 2013). Uterine NKs also colonize the uterine lining at or shortly after implantation and their production of TGF- $\beta$ may help with the generation and maintenance of Treg cells (Vacca et al., 2010). Both $\mathrm{E}_{2}$ and $\mathrm{P}_{4}$ upregulate the expression of uNK cell chemokines CXCL10 and CXCL11 in the endometrium (Sentman et al., 2004). TGF $\beta$ is expressed by the endometrium and maternal lymphomyeloid cells (Clark et al., 1997; Simpson et al., 2002; Gabrysová et al., 2011) and is involved in preparing the uterus for implantation by inducing expression of LIF (d'Hauterive et al., 2005) and GM-CSF (Robertson et al., 2002), which results in leukocyte recruitment (Tremellen et al., 1998). TGF $\beta$ also reduces IL-6 production (d'Hauterive et al., 2005) and inhibits Th1 responses (Eriksson et al., 2004).

TGF $\beta$ is a potent cytokine in its ability to limit inflammation and modify innate and adaptive immunity; however, its effects are dependent on context (Sporn, 1999) and the presence of other cytokines in the local environment. TGF $\beta$ acting on resting cells usually results in them being turned on/activated, whereas, inflammatory cells that are already active respond to TGF $\beta$ by being inhibited by the cytokine (Sporn, 1999). TGF $\beta$ can suppress activated MФ functions (Bogdan and Nathan, 1993; Chen and Wahl, 2002), suppress NO production (Vodovotz et al., 1993), influence $\mathrm{T}$ cell differentiation (Gorelik et al., 2002), and suppress effector T cell proliferation, cytokine responses, and cytotoxic effects 
by inhibiting granzyme-B and the transcription factors for Th1 (T-bet) and Th2 (GATA3) (Gorelik and Flavell 2000; Heath et al., 2000; Gorelik et al., 2000; Chen et al., 2003; Lin et al., 2005; Thomas and Massaqué, 2005).

TGF $\beta$ also suppresses cell proliferation by suppressing c-Myc and the cell cycle inhibitors p15 ${ }^{\mathrm{INK} 4 \mathrm{~b}}$ and $\mathrm{p} 21^{\mathrm{CIP} 1}$ (Wahl et al., 2004). IL-2 can perturb the inhibitory influence of TGF 3 (Chen and Wahl, 2002). IFNY is another cytokine that can alter TGF $\beta$ functions. By enhancing expression of inhibitory Smad7, IFNץ can block TGF $\beta$ signaling (Nakao et al., 1997; Strober et al., 1997). TGF $\beta$ can directly inhibit IFNY and IL-2 production by T cells or indirectly by affecting the ability of APCs to activate Th1 cells by suppressing CD40 expression, suppressing IL-12 production by APCs, and/or inhibiting IL-12 from activating Jak-Stat pathways (Strober et al., 1997; Bright and Sriram, 1998; Gortham et al., 1998). These examples of complex immunregulatory circuits demonstrate that the balance of pro and anti-inflammatory cytokines and how they interact will influence the generation and maintenance of Treg cells and tolerance at the feto-maternal interface.

Injecting TGF- $\beta$ into the vaginal tracts of female mice of abortion-prone matings that have a suboptimal Treg response to the male ejaculate promotes pregnancy success and is shown to recruit Treg cells to the vaginal tract (Clark et al., 2008). Seminal fluid has been shown to be the key regulator of the uterine Treg cell population by stimulating proliferation of Tregs and thus increasing the pool of available Treg cells and by promoting recruitment of Tregs from the circulation to the implantation site (Guerin et al., 2011). During copulation, sperm and seminal plasma are deposited into the female genital tract where they interact with resident MФs and DCs. Being APCs, the MФs and DCs pick up paternal alloantigen and carry it to the draining lymph nodes where stimulatory DCs can activate $\alpha \beta$ T cells to become effectors of rejection or tolerogenic DCs will activate Tregs (Clark and Chaouat et al., 2012). TGF $\beta$ in the seminal fluid is important for polarizing the DCs encountering paternal antigens into a tolerogenic phenotype, so that they induce Tregs in the lymph nodes. This is achieved by regulating which costimulatory molecules and cytokines are expressed by DCs (Robertson et al., 2002). DCs matured in vitro with TGF $\beta$ express low levels of the T-cell costimulatory molecules CD80, CD86, and CD40 and can exert a tolerant function in vivo (Lu et al., 1997). Once Treg cells have been activated and proliferated they will leave the lymph node and enter the circulation. Seminal fluid and sperm are also responsible for increasing the expression of the Treg chemokine CCL19 by glandular and luminal epithelial cells in the uterus. CCL19 interacts with the CCR7 receptor on Tregs to recruit and retain Tregs in peripheral tissues, in this case the uterus (Guerin et al., 2011). Other types of T cells that can cause fetal rejection also localize to the uterine lining but their function is kept in check by tDCs via IDO and the 
tryptophan metabolite 3-hydroxyanthranillic acid (3HAA) (can induce T cell apoptosis) along with TGF $\beta$ promoting Treg activity (Clark and Chaouat et al., 2012). Insufficient tDCs and/or increased amounts of stimulatory DCs can lead to fetal rejection.

\section{Interleukin-10}

Interleukin-10 (IL-10) is a critical anti-inflammatory cytokine involved in immune tolerance at the feto-maternal interface and controlling response to PAMPs. IL-10 inhibits COX-2 transcription induced by LPS (Niiro et al., 1995), thus limiting $\mathrm{PGE}_{2}$ production. This occurs through inhibition of STAT5 through suppression of LPS induced GM-CSF production (LPS->GM-CSF->STAT5->COX-2) (Yamaoka et al., 1998) and by preventing LPS activation of ERK2 and p38 MAPK in monocytes (Nirro et al., 1998). IL-10 blocks upregulation of TLR4 in response to LPS or PICs (Muzio et al., 2000), limiting the inflammatory response to LPS. IL-10 can inhibit MHC class II antigen expression on monocytes through inhibition of the transport of mature, peptide loaded MHC class II molecules to the plasma membrane (Koppelmann et al., 1997). IL-10 inhibits expression of co-stimulatory molecules, CD54 (ICAM), CD80, and CD86 by monocytes (de Waal Malefyt et al., 1991; Ding et al., 1993; Willems et al., 1994; Koppelmann et al., 1997). IL-10 also will affect MHC class II and co-stimulatory molecules expression by DCs as well as the cytokines produced by the DC (Moore et al., 2001). These effects will be detrimental to the ability of the APC to present antigen/stimulate T cells. T cell "activation" in the presence of IL-10 induces CD4+ T cells to become anergic in an antigen specific manner (Caux et al., 1994; Groux et al., 1996) that is not reversible with IL-2 or stimulation of CD3 and CD28 (Groux et al., 1996). These anergic cells will no longer react to the antigen they are specific for and can act as suppressor cells by competing with other antigen-activated T cells for IL-2 (Wan and Flavell, 2006).

IL-10 inhibits the activation of NFkB in activated monocytes/macrophages and the production of cytokines (IL-1 $\alpha$, IL-1 $\beta$, TNF $\alpha$, IL-6, IL-12, IL-18, GM-CSF, M-CSF, LIF, PAF) and chemokines (MCP1, MCP-5, Mip-1 $\alpha$, Mip1 $\beta$, Mip-3 $\alpha$, Mip-3 $\beta$, RANTES, MDC, IL-8, IP-10, MIP-2) by innate immune cells that recruit monocytes, DCs, neutrophils, and T cells (Moore et al., 2001; Akdis et al., 2011).

IL-1 and TNF $\alpha$ tend to have synergistic effects on inflammatory pathways inducing additional inflammatory mediators such as chemokines, PGs, and platelet activating factor (PAF). By inhibiting production of these two cytokines, IL-10 has significant anti-inflammatory effects. In addition, IL-10 enhances production of IL-1Ra (Moore et al., 2001), an endogenous blocker of IL-1 effects. IFNy induces IP-10 expression, which attracts Th1 cells, while IL-10 inhibits IFN $\gamma$ activated STAT molecules, which may occur through upregulation of suppressor of cytokine signaling 3 (SOCS3) (Cassatella et al., 1999; Ito et al., 1999; Yamaoka et al., 1999). By interfering with downstream IFN $\gamma$ signaling, IL-10 suppresses 
Th1 recruitment (Moore et al., 2001). The importance of IL-10 mediating the cytokine environment at the feto-maternal interface is shown by IL-10 null mice being more susceptible to LPS induced fetal loss as a result of increased expression of TNF $\alpha$, IL-6, IL-1, and IL-12 compared to LPS treated control mice (Robertson et al., 2007).

\section{Galectin-1}

Galectins are a family of $\beta$-galactoside-binding proteins involved in immunoregulatory functions. After release from a cell, galectins crosslink $\beta$-galactoside-containing cell-surface glycoconjugates, leading to changes in cell signaling, adhesion, and cell survival (Rabinovich et al., 2002). Gal-1 recognizes and binds multiple glucose- $\beta 1-4-\mathrm{N}$-acetylglucosamine (LacNAc) units located on branches of N-Linked or O-linked glycans (Rabinovich and Toscano, 2009).

Galectin-1 is expressed in reproductive tissues including the myometrium, decidua of the uterus, and invasive extravillous trophoblast (Phillips et al., 1996; Maquoi et al., 1997; Rabinovich, 1999; Sasaki et al., 2004; von Wolff et al., 2005; Kallikourdis et al., 2007; Blois et al., 2007b; Ramhorst et al., 2012; Than et al., 2012; Tirado-González et al., 2013) where it may play a role in controlling inflammation by triggering apoptosis of infiltrating effector T cells and reducing production of PICs (Rabinovich et al., 2002). It is produced by Tregs, MФs, and uNKs located at the fetomaternal interface (Rabinovich et al., 1998; Koopman et al., 2003; Kopcow et al., 2008). Expression by the placenta is likely involved in cell adhesion and invasion of the trophoblast, but gal-1 also plays an important role in maternal tolerance to placental alloantigens (Than et al., 2012).

With regard to modulating effector functions by other T cells, gal-1 interacts with surface glycoproteins on T cells and specifically CD45, CD43, and CD7 are involved in gal-1 induced apoptosis (Pace et al., 1999). Gal-1 inhibits production of IL-2, IFN $\gamma$, and TNF $\alpha$ from activated T cells and IL-12 from infected MФs; decreases release of $\mathrm{AA}$ and $\mathrm{PGE}_{2}$ production from $\mathrm{M} \Phi \mathrm{s}$; decreases $\mathrm{PLA}_{2}$ induced edema, neutrophil extravasation and mast cell degranulation; and causes cell-cycle arrest and/or apoptosis of activated peripheral T cells, and infected MФs (Perillo et al., 1995; Blaser et al., 1998; Rabinovich et al., 1999; Vespa et al., 1999; Rabinovich, 2000; Rabinovich et al., 2000; Rabinovich et al., 2002). Gal-1 influences $\mathrm{T}$ cell physiology and survival by initiating signal transduction events including ERK-2 phosphorylation, calcium influx, activation of the transcription factor AP-1, and downregulation of the anti-apoptotic protein BCL-2 (Vespa et al., 1999; Rabinovich et al., 2000). Binding of gal-1 to a T cell alone or with antigen stimulation causes partial phosphorylation of TCR-zeta and generation of inhibitory pp21zeta (Chung et al., 2000). Galectin-1 limits membrane reorganization and causes cross-linking events that limit the size of lipid raft clusters at the TCR contact site and raft-associated protein tyrosine 
phosphorylation. Because of these gal-1 actions, functions in the T cell that need only partial TCR signals, like apoptosis, can occur but functions that require co-stimulation or tyrosine phosphorylation, like IL-2 production, are blocked (Chung et al., 2000) meaning that the T cell cannot be activated or induced to proliferate.

Galectin-1 has been reported to be important for the generation of feto-maternal tolerance through involvement in the generation of tDCs and Tregs. Blois et al (2007b) showed that gal-1 deficient mice had higher rates of fetal loss during allogenic matings but not syngeneic matings. Treating gal-1 deficient mice with recombinant Gal-1 prevented fetal loss and restored tolerance. This was associated with a shift towards a Th2 dominant cytokine profile at the fetomaternal interface which is known to be crucial for successful pregnancy (Wegmann et al., 1993; Blois et al., 2007b). Galectin-1 expression may be regulated by $\mathrm{P}_{4}$ as Blois et al (2007b) reported that treatment with the $\mathrm{P}_{4}$ derivative dydrogesterone increased gal-1 expression that was correlated with expansion of Treg cells and DCs with a regulatory phenotype in the myometrium and decidua of stress-challenged pregnant mice. $\mathrm{P}_{4}$, and gal-1 may work in a reciprocal manner as treatment with gal-1 prevented the stress-induced decrease in $\mathrm{P}_{4}$ and in fact increased systemic $\mathrm{P}_{4}$ levels higher than amounts measured in non-stressed pregnancies (Blois et al., 2007b). Choe et al (1997) also reported that gal-1 is under steroid hormone control, and uterine expression is particularly high around the time of implantation in the mouse.

Galectin-1 control over T helper cytokine profiles may be due to susceptibility of the different subsets to Gal-1 induced apoptosis (Motran et al., 2008). Signaling and subsequent cell death by gal-1 is dependent not only on clustering and segregation of CD45 with CD3 and CD43 with CD7 on the target cell (Pace et al., 1999), but on the glycosylation status of the glycoproteins (Nguyen et al., 2001; Amano et al., 2003; Hernandez et al., 2006; Toscano et al., 2007). Th1 and Th17 cells express the surface glycans that Gal-1 binds to cause apoptosis, Th2 cells, however, are protected from Gal-1 through differential sialylation of their cell surface glycoproteins (Toscano et al., 2007; Kopcow et al., 2008; Rabinovich and Ilarregui, 2009; Ramhorst et al., 2012b). Galectin-1 also will induce activated CD8+ T cells to undergo apoptosis (Kopcow et al., 2008).

Recombinant gal-1 induces DCs to have a tolerogenic phenotype. The tDCs then promote expansion of Tregs characterized by production of IL-10. Tregs play an essential role in the generation of feto-maternal tolerance by influencing the types of cytokines produced and suppressing proliferation of CD25- T cells. Tregs are generated in an antigen specific manner and create an overall immunosuppressive local environment where that antigen is expressed. This is important at the fetomaternal interface because the mother needs to tolerate the paternal antigens expressed by the fetus (Aluvihare et al., 2004; Sasaki et al., 2004; Somerset et al., 2004; Kallikourdis et al., 2005) The 
importance of Tregs was shown when feto-maternal tolerance failed to be generated in mice depleted of Tregs or deficient in IL-10, even with the addition of Gal-1. This result also demonstrates that one of the mechanisms of Gal-1 in generating tolerance is through generation of Tregs (Blois et al., 2007b; Ramhorst et al., 2012b) and inducing production of IL-10 from T lymphocytes (van der Leij et al., 2004).

Regulatory T cells also produce Gal-1 and expression is upregulated upon TCR activation. It plays a role in the suppressive function of Treg cells as anti-gal-1 antibody reversed the suppressive function of human and mouse Tregs in vitro and Tregs isolated from gal-1 null mice also had reduced suppressive capabilities (Garín et al 2007). Tregs use the molecule to control other types of T cells (Garín et al., 2007). Gal-1 inhibits cytokine production from Th1 and Th17 cells, induces cytokine production from Th2 and Treg cells and inhibits adhesion and transendothelial migration of effector T cells (Norling et al., 2008; Rabinovich and Ilarregui, 2009; Ramhorst et al., 2012b).

Galectin-1 expression is increased during an inflammatory response (Ilarregui et al., 2009) and expression has been shown to be induced by NF-kB (Toscano et al., 2011). A regulatory mechanism to control the inflammatory response is built in by gal-1 being able to inhibit activation of NF-kB (Toscano et al., 2011).

\section{Dendritic Cells}

The phenotype of DCs is important because they can either be immunogenic and cause fetal loss or promote immune tolerance at the feto-maternal interface (Kämmerer et al., 2003; Miyazaki et al., 2003; Blois et al., 2005; Arck et al., 2007), which is dependent on the signals they receive and the cytokines they produce when presenting antigen. $\mathrm{tDCs}$ are either immature, maturation resistant, or alternatively activated DCs (activated after exposure to corticosteroids or anti-inflammatory cytokines). These cells express MHC molecules, inhibitory molecules, low levels of co-stimulatory molecules (CD40, CD0, CD86), have low production of PICs, produce IL-10, and do not synthesize Th1 polarizing cytokines (IL12p70) (Arck et al., 2007). tDCs not only play an important role in the generation of Tregs but are involved in the shift towards a predominantly $\mathrm{Th} 2$ cytokine profile at the feto-maternal interface (Miyazaki et al., 2003).

The low ratio of co-stimulatory to inhibitory signals makes it so that when tDCs present antigen, there is inadequate co-stimulation to activate and cause proliferation of effector T cells (instead they undergo apoptosis or become anergic), but tDCs can cause the generation and expansion of Treg cells (Morelli and Thomson, 2007). Treg cells are end-stage effector cells that arise from differentiation away from the alternative Th17 effector pathway, and this is influenced by TGF- $\beta$ and IL-6. While IL-6 influences activated CD4+ T cells to become Th17 cells, TGF- $\beta$ plus IL- 2 can prevent IL- 6 from 
converting iTregs cells into Th17 type T cells by down-regulating IL-6 receptor expression (Zheng et al., 2008). While the polarization of naïve CD4+ cells into T help cell subsets is generally viewed as irreversible, this may not be true for Treg and Th17 cells. These two T helper cell subsets are less stable and have greater plasticity in switching between the two phenotypes if environmental conditions change (Zhou et al., 2009). This means an infection or other insult that increases PICs can result in a shift from Treg cells to Th17 cells that would be detrimental to pregnancy.

Ilarregui et al (2009) reported that Gal-1 induces DCs to produce IL-27 which subsequently promotes DCs to be tolerogenic, to produce IL-10, and to cause the generation of induced T regulatory type $1(\mathrm{Tr} 1)$ cells (lack FOXP3 expression) that also produce IL10 to regulate T cell function. The importance of IL-27 from DCs in causing the differentiation of naïve CD4+ T cells into IL-10 producing $\operatorname{Tr} 1$ cells was reported by Awasthi et al (2007); TGF $\beta$ was also reported to amplify the generation of $\operatorname{Tr} 1$ cells by IL-27.

Interleukin-27 producing tDCs were generated from interaction with Tregs, most likely in response to gal-1 produced by the Tregs (Awasthi et al., 2007; Garín et al., 2007). The cytokine induces the transcription factors AhR and c-Maf, which bind together to promote transactivation of the IL-10 and IL-21 promoters. The DC then uses these cytokines to cause the generation of Tr1 cells (Apetoh et al., 2010). IL-21 has also been shown to induce IL-10 synthesis via STAT3 phosphorylation, and can mediate IL-27 induced IL-10 production, modifying the plasticity of committed Th17 cells (Spolski et al., 2009.) IL-27 promotes the differentiation of IL-10 producing Treg cells while inhibiting generation of Th17 cells by inhibiting IL-17 secretion and other molecules associated with the function and maintenance of a Th17 phenotype (Murugaiyan et al., 2009).

Activated Th cells exposed to gal-1 increase expression of the surface-bound immunomodulatory molecules CD25, CTLA-4, and PD-1, produce IL-10, and stop producing IFNץ and IL-17, essentially turning them into Tr1 cells (Cedeno-Laurent et al., 2012). Gal-1 treated cells had increased phosphorylation of STAT1 and STAT3, which have already been established as being associated with $\operatorname{Tr} 1$ formation (Wang et al., 2011). IL-10 production in response to gal-1 was associated with expression of the transcription factors c-Maf, AhR and Jun-B (mediates IL-10 expression in Th2 cells (Wang et al., 2005)) (Cedeno-Laurent et al., 2012). C-Maf has already been identified as a crucial transcription factor in Tr1 cell differentiation (Pot et al., 2009). Th1 and Th17 cells have been seen to be susceptible to gal-1 mediated apoptosis at concentrations $>7 \mu \mathrm{M}$ (Toscano et al., 2007), thus there may be two consequences to Th1 and Th17 exposure to gal-1 depending on the exposure amount. Consequence 1 is IL-10 synthesis during differentiation or after commitment to Th subtypes through the c-MAF/AhR/IL-21 pathway 
(Cedeno-Laurent et al., 2007) and consequence 2 is cell death at high concentrations (Toscano et al., 2007). IDO expression by tDCs can also induce apoptosis of activated effector T cells (Th1 and Th17) and is involved in promoting Treg generation through the generation of 3-hydroxyanthranillic acid, a tryptophan metabolite (Morelli and Thomson, 2007).

\section{Progesterone}

Progesterone is produced by the $\mathrm{CL}$ and by the placenta to varying degrees depending on the species. The steroid hormone is known to have immunosuppressive affects and plays a role in modulating the immune environment in the uterus. $\mathrm{P}_{4}$ has genomic and non-genomic effects with non-genomic effects being mediated by G-protein coupled receptors and genomic effects being mediated by two isoforms of its nuclear receptor (PR-A and PR-B) that act as transcription factors (Li et al., 2004; Mulac-Jericevic and Conneely, 2004; Arck et al., 2007). In the peri-implantation uterus, $\mathrm{P}_{4}$ has been reported to regulate several genes including amphiregulin (in the EGF superfamily), Hox-A10 and Hox-A11 (homeobox gene family), histidine decarboxylase (histidine-> histamine), calcitonin, immune response gene-1 (Irg-1), Muc1, Indian hedgehog, and gal-1 (Choe et al., 1997; Arck et al., 2007). Progesterone can influence cytokine production as well as immune cell function.

In pregnant women, $\mathrm{P}_{4}$ causes the production of P-induced blocking factor (PIBF) that mediates some of progesterone's immunological effects (Arck et al., 2007). PIBF activates the JAK/STAT pathway activating STAT6, which then causes transcription of SOCS-3. SOCS-3 will bind to the IL-12R, inhibiting IL-12 signaling and preventing IL-12 activation of STAT4 (Kozma et al., 2006), thus influencing T cells towards a Th2 phenotype and blocking a Th1 phenotype. PIBF therefore, leads to $\mathrm{T}_{\mathrm{H}} 2$ dominant cytokine production (Szekeres and Wegmann, 1996) and blocks NK cell activation because of the reduced IL-12 production (less Th1 cells) (Szekeres-Bartho et al., 1996). In sheep, the $\mathrm{P}_{4}$ induced immunoregulatory molecule is not PIBF but instead a member of the serine proteinase inhibitor family called uterine serpin. This molecule is also secreted from the endometrium of goats, cattle, and pigs and has been reported to block lymphocyte proliferation in vitro (Liu et al., 1999; Peltier et al., 2000).

Murine studies have shown that CD4+ T cells contain the nuclear PR, and $\mathrm{P}_{4}$ reduces effector activity of these cells through binding of its nuclear receptor and repressing transcription of IFN $\gamma$ (Hughes et al., 2013). Also, through interaction with its nuclear receptor, $\mathrm{P}_{4}$ has been shown to promote the generation of murine induced Treg cells in vitro that have suppressive functions with regards to generation of effector T cells and inflammation in vitro and in vivo (on target T cells) (Lee et al., 2012). $\mathrm{P}_{4}$ suppresses mTORC1 signaling which is needed for the generation of Th1 and Th17 cells; in its absence, Treg cells are produced (Haxhinasto et al., 2008; Lee et al., 2012). $\mathrm{P}_{4}$ also leads to the expansion of the Treg population during murine pregnancy (Mao et al., 2010). 
Culture of human peripheral blood mononuclear cells (PBMCs) from pregnant women with $\mathrm{P}_{4}$ resulted in reduced production of IFN $\gamma$ and TNFa, while increasing production of IL-4 (Lissauer et al., 2015). $\mathrm{P}_{4}$ is a potent inducer of Th2 cytokines (Piccinni et al., 1995; Miyaura and Iwata, 2002) including IL-4 which mediates $\mathrm{P}_{4}$ stimulated LIF (Piccinni et al., 1998) and M-CSF (Piccinni et al., 2001) producing T cells. LIF is critical for implantation as it mediates uterine receptivity (Rosario and Stewart, 2016) while M-CSF is important for pregnancy development (Piccinni et al., 1998; Piccinni et al., 2001; Song et al., 2009). The Th2 cytokines, IL-4 and IL-10, can inhibit development and function of Th1 cells and MФs, which is important as IL-12 and IFN $\gamma$ downregulate LIF and a Th1 response promotes allograft rejection (Piccinni et al., 1998; Piccinni et al., 2001).

Progesterone can regulate mature DC function and DC-stimulated proliferation of T cells in a receptor-mediated manner (Butts et al., 2007). Treatment of LPS activated mature bone marrow derived dendritic cells (BMDCs) with $\mathrm{P}_{4}$ did not alter antigen take up by DCs, but suppressed production of the PICs TNF $\alpha$ and IL-1 $\beta$ without affecting IL-10 production. Treatment with $\mathrm{P}_{4}$ also down regulated expression of the co-stimulatory molecule CD80 by DCs and inhibited DC-stimulated T cell proliferation (Butts et al., 2007). As the major APC in the uterus, reduced PIC production and decreased expression of co-stimulator molecules by DCs will limit Th1 responses and T cell responses to fetal antigen.

Acting through the $\mathrm{GC}$ and $\mathrm{P}_{4}$ receptors, $\mathrm{P}_{4}$ can downregulate innate immune responsivity of $\mathrm{M} \Phi \mathrm{s}$ by modulating TLR signaling (Jones et al., 2008; Su et al., 2009). The downregulation is associated with the suppression of NF-kB activation and enhancement of SOCS1 expression leading to a decrease in NO, IL-6, and IL-12 production (Miller et al., 1996; Su et al., 2009).

Mucin-1 expression is induced by $\mathrm{E}_{2}$ and expressed in humans by the syncytiotrophoblast at the feto-maternal interface and by the extravillous trophoblast cells invading the decidua (Jeschke et al., 2002). Mucin-1 is thought to control the correct placement and timing of implantation. Blastocyst implantation is proposed to be regulated by a uterine barrier consisting of a high density of Muc-1 at the epithelial cell surface that inhibits blastocyst adhesion (Aplin et al., 2001). Downregulation of Muc-1 expression by the uterine epithelium at the implantation site during the window of implantation allows for blastocyst adhesion. This may be regulated by $\mathrm{P}_{4}$ antagonizing Muc1 expression (Brayman et al., 2006).

\section{Complement}

The complement system is part of the innate immune response and is involved in killing pathogens, inflammation, production of PICs, chemotaxis of inflammatory cells, opsonisation and clearance of immune complexes (Molina, 2005; Murphy 2012). Proper control of complement in the uterine environment is necessary to prevent inflammation and embryo loss since inappropriate 
complement activation results in loss of maternal-fetal tolerance (Xu et al., 2000; Mellor et al., 2001; Caucheteux et al., 2003; Mao et al., 2003). There are 3 complement pathways that differ in how the pathway initiates, but all converge at the formation of $\mathrm{C} 3$ convertase. The classical pathway is initiated when complement proteins bind antigen-antibody complexes; the lectin pathway is initiated when mannose-binding lectin binds to mannose residues in microbial proteins and polysaccharides, and the alternative pathway is initiated when complement fragments bind directly to the microbial cell wall. The pathways end with the formation of the membrane attack complex, which is inserted into the wall of a target cell resulting in it being lysed. During the complement cascade some complement fragments have other effects rather than being involved in formation of the convertases or membrane attack complex. These complement fragments (C3a, C3b, C4a, C5a) have effects such as opisonization of target cells, causing degranulation of mast cells, basophils, and eosinophils, extravasation and chemotaxis of leukocytes to the inflammatory site, aggregation of platelets, release of hydrolytic enzymes from neutrophils, and increased expression of complement receptors by neutrophils (Murphy, 2012).

Murine studies have shown the role of complement deposition in fetal loss, abnormal placental development and the importance of complement regulation in the uterine environment to prevent complications (Xu et al., 2000; Wu et al., 2000; Mao et al., 2003). Girardi et al (2006b) reported that C3 deposition in the decidua of mice was associated with increased necrotic tissue, fetal debris, and infiltration of inflammatory cells such as neutrophils and monocytes. Xu et al (2000) demonstrated that deficiency in the complement regulatory protein Crry (complement receptor 1-related gene/protein y) in mice results in fetal loss due to spontaneous $\mathrm{C} 3$ deposition in the placenta at the fetomaternal interface and embryos being invaded by polymorphonuclear cells. Complement fragments cause increased production/release of PICs, such as TNF $\alpha$ (Girardi et al., 2006b). Increased TNF $\alpha$ levels at the fetomaternal interface can lead to fetal loss as TNFa can reduce inner cell mass proliferation, induce trophoblast apoptosis and restrict trophoblast invasiveness (Hunt et al., 1989; Yui et al., 1994; Pamofer et al., 1994a; Pampfer et al., 1994b; Silver et al., 1994; Pampfer et al., 1995). Fetal loss in Crry deficient mice was attributed to $\mathrm{C} 3$ activation because Crry null mice pups were born only if they were on a C3 deficient background (Xu et al., 2000) Crry is a membrane bound protein that inhibits the deposition of activated $\mathrm{C} 3$ and $\mathrm{C} 4$ on the surface of autologous cells and has been shown to be expressed in trophoblasts and maternal decidual tissues. While Crry is specific to mice, other complement regulatory proteins that have been found in high levels in human placentas, such as decay accelerating factor (DAF/CD55) and membrane cofactor protein (MCP/CD46) may have a similar role in other species (Caucheteux et al., 2003). 
Female mice of abortion prone matings were reported to have increased complement activation that was associated with impaired placental vascularization and a deficiency in the number of trophoblast giant cells (Girardi et al 2006a). Trophoblast giant cells mediate implantation and invasion of the decidua by the conceptus; efficient numbers of these cells leads to placental failure (Kraut et al., 1998; Cross et al., 2005). Placental development requires angiogenic growth factors, VEGF and placenta growth factor along with expression of their receptors (Lam et al., 2005). By binding to the tyrosine kinase receptors VEGFR-1 (Flt-1) and VEGFR-2, VEGF promotes placental development and invasiveness (Ferrara et al., 2003). The VEGFR-1 can be alternatively spliced to produce a secreted form of the receptor (soluble sVEGFR-1/sFLT-1) that lacks the cytoplasmic and transmembrane domains of the receptor but retains the ligand binding domain (Kendall and Thomas, 1993). This secreted form of the receptor is actually antiangiogenic by binding and sequestering circulating VEGF and placental growth factor, preventing the molecules from interacting with their endogenous receptors (He et al., 1999). Impaired placental vascularization in abortion prone mice matings in Girardi et al's study (2006a) was found to be due to C5a produced during the complement cascade inducing infiltrating monocytes to produce sVEGFR-1 that then bound VEGF inhibiting its activity during placental development. Thus, complement can affect the pro-angiogenic functions of uterine $\mathrm{M} \Phi$ s impairing pregnancy success.

\section{Bacterial Infections and the Innate Immune System}

Although there are numerous mechanisms in place to generate and maintain maternal tolerance to the fetus (Trowsdale and Betz, 2006), an acute or chronic uterine infection during pregnancy that activates the immune system to clear the infection can also result in disruption of maternal tolerance and ultimately fetal loss (Mor, 2008; Koga et al., 2014). Infection induced loss is most likely to occur during the early phases of pregnancy when maternal tolerance is still being generated. Paternal alloantigens presented during a uterine infection are likely to be presented in the presence of PICs, which will favor activation of inflammatory/effector $\mathrm{T}$ cells instead of Tregs, which can result in fetal rejection (Trowsdale and Betz, 2006).

A uterine infection will cause inflammation by signaling innate immune cells through PAMPs and DAMPs; LPS even increases expression of the IFN $\gamma$ and TNF $\alpha$ receptors heightening the inflammatory response. This can result in a shift in cytokine expression, a switch from tolerogenic to immunogenic DCs, a loss in Treg cell function, and uNKs to become cytotoxic, ultimately resulting in fetal loss. Exposure to a PAMP or DAMP can also cause MФs, trophoblast cells, and endothelial cells to up-regulate surface expression of a membrane pro-thrombinase (Fibrinogen-like protein 2/mFGL2) that will activate thrombin and result in fibrin deposition, vascular thrombosis, and inflammation. Once thrombin is activated there is positive feedback for further activation. Thrombin will activate complement 
component 5 which will spilt into C5a and C5b (Clark and Chaouat et al., 2012). C5a will then act as a chemoattractant to bring in additional innate immune cells and cause release of inflammatory mediators (Murphy, 2012). The combination of polymophonuclear leukocytes recruitment by endothelial cells and C5a, inflammation, and fibrin deposition will impair the vascular supply to the placenta and cause fetal loss (Clark and Chaouat et al., 2012).

DC maturation results in upregulation of surface expression of MHCII, CD80, CD86, CD40, CCR7, and production of cytokines like IL-12 and TNFa. Maturation occurs in response to DAMPs, PAMPs, and PICs (Morelli and Thomson, 2007). Monocyte derived DCs that mature in the presence of $\mathrm{PGE}_{2}$ are induced to migrate to secondary lymph nodes (Luft et al., 2002). This effect maybe mediated by $\mathrm{PGE}_{2}$ altering CCR7 signal transduction (Scandella et al., 2004). This chemokine receptor responds to the ligands CCL19 and CCL21, which are expressed in lymphoid organs (Scandella et al., 2004). An infection that increases PICs and $\mathrm{PGE}_{2}$ will result in DCs traveling to lymph nodes to present antigen while producing Th1 polarizing cytokines.

Regulatory $\mathrm{T}$ cells control NK cell proliferation, secretory function, and cytotoxic activity in a TGF $\beta$ dependent manner involving downregulation of the NKG2D receptor (receptor on the surface of NK cells, cytotoxic CD8+ cells, and $\gamma \delta \mathrm{T}$ Cells; ligand binding leads to perforin-mediated cytotoxicity), insufficient quantity of Treg cells can result in an overabundance of NK cell activity (Ghiringhelli et al., 2005a). Treg cells, however, cannot suppress NK cell function when the IL-2RY chain (cytokine receptor sub unit that is a part of the receptors for IL-2, IL-4, IL-7, IL-9, IL-15, or TLR4 is activated (Ghiringhelli et al., 2005a). This means that with an acute infection or LPS exposure, Tregs will be unable to prevent NKs from proliferating and having cytotoxic activity. With regards to pregnancy, a uterine infection will likely convert specialized uNK cells into cytotoxic NKs that behave similarly to their peripheral counterparts resulting in pregnancy loss.

Infection can be a stressful event and can involve the activation of the hypothalamic-pituitaryadrenal (HPA) axis. High levels of GCs exert adverse effects on the uterus and fetus and inhibit LH, $\mathrm{E}_{2}$ and $\mathrm{P}_{4}$ secretion (Magiakou et al., 1997). Stressful events during pregnancy can also challenge the immune suppressive environment of the uterus leading to pregnancy loss (Arck et al., 1995). Allogenically mated mice exposed to stress early in gestation causes a decrease in $\mathrm{P}_{4}$ (Joachim et al., 2003), increased secretion of PICs (Blois et al., 2004b), migration of inflammatory cells to the fetomaternal interface (Blois et al., 2005), and decreased Treg cells and gal-1 (Blois et al., 2004b; Blois et al., 2007b), thus resulting in a switch from an anti-inflammatory to a pro-inflammatory environment. 
The trophoblast expresses PPRs that sense bacteria, viruses, dying cells, and damaged tissue in the surrounding environment (Abrahams et al., 2005; Mor et al., 2005, Costello et al., 2007). In response to PAMPs and DAMPs, the trophoblast will secrete cytokines that will act on immune cells (MФs, DCs, Tregs, NK cells) in the uterus and recruit additional immune cells (Fest et al., 2007). Immune cells in the pregnant uterus tend to have a specialized phenotype: NK cells are not cytotoxic, DCs are tolerogenic, T cells are regulatory; however, infections can cause them to lose that phenotype. While they were promoting fetal tolerance, with an infection, they switch to functions that promote fetal rejection (Murphy et al., 2005; Fest et al., 2007; Mor, 2008). The trophoblast can respond to PICs in addition to producing them, and an increase in PICs can change trophoblast resistance to Fas-mediated apoptosis leading to embryonic death (Abrahams et al., 2004). There are 3 major ways for a viral or bacterial infection to gain access to the uterus: through the maternal circulation, by ascending into the uterus from the lower reproductive tract (most common), or by descending into the uterus from the peritoneal cavity (Deb et al., 2004; Espinoza et al., 2006).

\section{Inflammation}

Inflammation is a mechanism to restore homeostasis in response to infection or injury but also after physiological changes in tissue architecture like ovulation or parturition (Medzhitov, 2008; Sheldon et al., 2014). Classical signs of inflammation are redness, swelling, heat, and pain. There are seven groups of inflammatory mediators: vasoactive amines, vasoactive peptides, complement fragments, lipid mediators, cytokines, chemokines, and proteolytic enzymes (Medzhitov, 2008). Vasoactive amines, such as histamine and serotonin, are released from granules in mast cells causing increased vascular permeability and vasodilation or vasoconstriction depending on the situation. Vasoactive peptides (substance $\mathrm{P}$, fibrinopeptide $\mathrm{A}$, fibrinopeptide $\mathrm{B}$, fibrin degradation products) cause vasodilation and increased vascular permeability directly or by causing histamine release from mast cells. Fragments generated during the complement pathways (C3a, C4a, C5a) trigger the synthesis of cytokines, chemokines, and adhesion molecules, promote granulocyte and monocyte recruitment and cause mast cell degranulation (granule contents then affect vasculature) (Medzhitov, 2008; Dinarello, 2010). Lipid derivatives (eicosanoids, platelet-activating factors, resolvins) are produced from phospholipids located in cell membranes.

Activated PLA2 releases arachidonic acid (AA) from membranes to be turned into eicosanoids. Cyclooxygenases (COX 1 and COX2) turn AA into PGs and thromboxanes while lipoxygenases turn AA into leukotrienes (LTs) and lipoxins (LXs) (Medzhitov, 2008). Some prostaglandins cause vasodilation ( $\mathrm{PGE}_{2}$ and $\mathrm{PGI}_{2}$ ), and $\mathrm{PGE}_{2}$ is hyperalgesic and causes fever (Higgs et al., 1984). Lipoxins along with 
resolvins and protectins produced from dietary omega-3 fatty acids inhibit inflammation and aid in the resolution of inflammation and tissue repair (Serhan, 2007).

Cytokines (interleukins, interferons, adipokines, tumor necrosis factors, mesenchymal growth factors) can be separated into groups based on their primary function: cytokines that are mostly lymphocyte growth factors, ones that are mostly pro-inflammatory, ones that are mostly antiinflammatory, or cytokines that polarize the immune response to antigen. Cytokines activate endothelium and leukocytes, cause fever, resistance to viral infections, lead to elevated white blood cell counts, and induce the acute phase response. Chemokines are responsible for controlling leukocyte extravasation and chemotaxis of additional immune cells to the affected area (Dinarello, 2007; Medzhitov, 2008). Proteolytic enzymes (elastin, cathepsins, matrix metalloproteinases) degrade extracellular matrix and basement membrane proteins, which is important for allowing leukocytes to move through the area and during tissue remodeling (Medzhitov, 2008).

The beginning of the inflammatory response is characterized by pro-inflammatory mediators (PIMs) (IL-1 $\beta$, IL-6, TNF $\alpha$, IL-8, PGE 2 ) that attract and activate immune cells like neutrophils and MФs to remove the pathogen and damaged cells. After the pathogens have been cleared, mediators like IL-10 (suppresses IFN $\gamma$, IL-1, TNFa, IL-6) and resolvins are released to stop the inflammatory reaction and to repair any damaged tissue (Dinarello, 2007; Sheldon et al., 2014). While inflammation is an important mechanism for restoring tissue homeostasis and is an important part of the innate immune system, chronic inflammation has adverse effects.

\section{Infection}

Infection is an important trigger for inflammation that is harmful to pregnancy and can lead to embryonic loss. There are PRRs located at the maternal-fetal interface (expressed on immune cells, trophoblast, epithelial cells, stromal cells) that recognize and bind PAMPs, which are unique to the surface of microorganisms (Martinon et al., 2009; Schroder and Tschopp, 2010; Murphy, 2012). Binding of these receptors to pathogen molecules results in the activation of anti-pathogen responses, such as the synthesis and secretion of chemokines, PICs, antimicrobial peptides, and acute phase proteins (APPs) (Wira et al., 2005; Akira et al., 2006; Davies et al., 2008; Medzhitov, 2008; Koga et al., 2014). PAMPs are typically molecules that are vital for microbial survival; therefore, they are unlikely to change because that would be detrimental to the microbe. Examples include bacterial structural components, such as LPS and peptidoglycan, and viral nucleic acids. PAMPS can be specific to classes of microbes and their invariance makes them a good way of alerting the innate immune system of a microbe's presence (Martinon et al., 2009). 
Toll like receptors (TLRs) are one family of innate immune system receptors with 10 members, some of which are located in the endosome and some are displayed on the cell surface. The TLRs that respond to bacteria are TLR2, which forms heterodimers with TLR1 and TLR6 and recognizes bacterial lipopeptides, and TLR4, which recognizes the gram negative bacterial cell wall component, LPS in complex with CD14 and MD2 (Chow et al., 1999; Saito et al., 2000; Abrahams, 2008; Takeuchi and Akira, 2010). LPS is an endotoxin located in the cell walls of gram-negative bacteria and consists of mostly carbohydrates and lipids. LPS has a hydrophilic core of repeating polysaccharides attached to an O-specific chain and the hydrophobic lipid-A domain (Raetz, 1993). The core region is conserved between different types of gram negative bacteria, whereas, the O-specific chain of repeating polysaccharides has some structural diversity. The lipid A region is the portion recognized by other cells and is responsible for LPS-induced biological responses (Rietchel et al., 1994). Endotoxins are constantly shed into the bacteria's environment, released after gram negative bacteria are ingested by phagocytes and degrade in vacuoles, and released when the bacterium lyse or disintegrate (Deb et al., 2004). The endotoxin can then interact with local host cells and/or travel in the blood to cause a variety of responses (Deb et al., 2004). In the circulation, LPS is bound to lipoproteins or LPS-binding proteins (LBP).

Not only are neutrophils, MФs, and DCs able to respond to PAMPs and DAMPs but other nonimmune cells also have PRRs. Endometrial epithelial and stromal cells express the TLR4/CD14/MD2 receptor complex and respond to LPS by secreting IL-6, CXCL8, and PGE 2 (Herath et al., 2006; Herath et al., 2009b; Cronin et al., 2012). In response to binding its ligand, TLRs initiate production and release of PICs or type I interferons (IFN) and IFN-inducible proteins (Akira and Hoshino, 2003; Koga et al., 2014). TLRs responding to bacterial components with the help of MYD88 stimulate IL1R associated kinases (IRAK4/IRAK1), which activate TNFR associated factor 6 (TRAF6) which catalyzes a complex consisting of TGF $\beta$ activated kinase 1 (TAK1) and members of the TAK1 binding protein (TAB) family. This complex can activate the MAPK pathway (JNK, ERK1/2, p38) leading to activation of the transcription factor AP-1 or it can activate the IKK complex (IKK $\alpha, \operatorname{IKK} \beta$, IKK $\gamma$ ) causing phosphorylation and ubiquitination of IкB. Ubiquitination of IкB causes degradation of the protein which releases NF-кB from the complex allowing it to translocate into the nucleus. In the nucleus, the transcription factors AP-1 and NF-kB activate transcription of PIMs including cytokines (IL-1 $\alpha$, IL-1 $\beta$, IL-6, TNF $\alpha$ ), chemokines (CXCL8, CXCL1), and lipid mediators (PLA2, PGHS, PGES). On the other hand, endosomal TLR4 recognizes viral envelope glycoproteins and signals in a MyD88 independent pathway that involves Toll/IL-1 receptor domain-containing adaptor inducing IFN- $\beta$ (TRIF). TRIF then either leads to activation of NF-kB for PIC production or IFN regulatory factor-3 (IRF-3) for type I IFN and IFN-inducible genes production (Takeuchi et al., 2000; Yamamoto et al., 2003). 
Prostaglandins are inflammation mediators and are produced in a cell-specific manner. Activation of the transcription factor NF-kB by IL-1 or TNF $\alpha$ leads to COX2 gene expression and the production of $\mathrm{PGE}_{2}$ (Dinarello, 2007). Monocytes/MФs produce large amounts of $\mathrm{PGE}_{2}$ and $\mathrm{PGF}_{2 \alpha}$ upon stimulation while neutrophils produce moderate amounts of $\mathrm{PGE}_{2}$, and mast cells produce $\mathrm{PGD}_{2}$. The enzyme 5-LOX is expressed by mast cells, monocytes, MФs, and granulocytes and converts AA into the 4-series LTs and hydroxyl and hydroperoxy derivatives (5-HETE and 5-hydroperoxyeicosatetraenoic acid (5-HPETE)) (Calder, 2003).

Prostaglandin $\mathrm{E}_{2}$ is involved in acute inflammation by inducing fever, lowering pain thresholds, promoting local vasodilation/vascular permeability and by promoting local attraction/activation of neutrophils, MФs, and mast cells (Calder, 2003; Dinarello, 2010; Kaliński, 2012). However, $\mathrm{PGE}_{2}$ also is considered an immunosuppressive product of inflammation. $\mathrm{PGE}_{2}$ can induce IL-10 production and can reduce levels of PICs, chemokines, and adhesion molecules reducing nonspecific and chronic inflammation (Dinarello, 2010; Kaliński, 2012).

Not only does $\mathrm{PGE}_{2}$ recruit cells to the local inflammatory site, but it also can affect their function. With regards to NK cells, $\mathrm{PGE}_{2}$ can suppress their ability to respond to IL-12 and IL-15 (Joshi et al., 2001; Walker and Rotondo, 2004), which results in suppressed cytolytic function (Bankhurst, 1982; Goto et al., 1983), and inhibit NK cell production of IFN $\gamma$, which is used to help DC cells induce Th1 and cytotoxic T cell responses (Mailliard et al., 2005). $\mathrm{PGE}_{2}$ can reduce phagocytosis and release of lysosomal enzymes by granulocytes (Smith, 1977).

$\mathrm{PGE}_{2}$ is a potent suppressor of $\mathrm{T}$ cell function. $\mathrm{PGE}_{2}$ increases intracellular cAMP levels, which then suppresses IL-2, IFN $\gamma$, and TNF $\alpha$ production (Dinarello, 2007; Dinarello, 2010). PGE 2 not only inhibits IL-2 production (Walker et al., 1983) but also the expression of the IL-2 receptor and JAK3 needed for IL-2 responsiveness by T Cells (Rincón et al., 1988, Kolenko et al., 1999), thus suppressing T cell activation and proliferation. DCs that mature in the presence of $\mathrm{PGE}_{2}$ are characterized with a phenotype of impaired ability to induce cytotoxic T lymphocytes, Th1, and NK cell mediated immunity (Kaliński et al., 1998; Kaliński et al., 1999; Gustafsson et al., 2008) but instead promote Th2 responses (Kaliński et al., 1999). $\mathrm{PGE}_{2}$ is able to suppress production of the Th1 polarizing cytokine IL-12 in monocytes (van der Pouw Kraan et al., 1995) and DCs (Kaliński et al., 1997; Kaliński et al., 1998) and can suppress IL-12 receptor expression and responsiveness (Wu et al., 1998). More directly, $\mathrm{PGE}_{2}$ inhibits Th1 IFNy production but does not affect Th2 cytokines, IL-4 and IL-5, by CD4+ T cells (Betz and Fox, 1991; Snijdewint et al., 1993). PGE 2 can cause development of Th17 cells through suppression of IL-12, which is inhibitory to Th17 polarization and by enhancing IL-23, which promotes Th17 
polarization (Kahyrullina et al., 2008). The PG also increases IL-17 production and augments IL-6 production (Calder, 2003; Dinarello, 2007; Dinarello, 2010). While Th17 cells are inflammatory, PGE $_{2}$ also promotes the development of Tregs from naïve T cells (Baratelli et al., 2005) and the interaction of DCs with Tregs (Muthuswamy et al., 2008), which may promote Treg expansion (Banerjee et al., 2006). $\mathrm{PGE}_{2}$ can also affect chemokine expression at the inflammatory site so that pro-inflammatory type MФs, cytotoxic T lymphocytes, NK cells, and Th1 cells are not attracted to the area (McIlroy et al., 2006; Gustafsson et al., 2008; Muthuswamy et al., 2008; Van Elssen et al., 2011), but Th2 and Tregs cells are attracted (McIlroy et al., 2006; Muthuswamy et al., 2008).

Neutrophils are usually the first responders to arrive at the site of an invading pathogen (Sadik et al., 2011). They are recruited from the vasculature to the site of pathogen invasion to aid in containing and clearing the pathogen (Chiang et al., 2012). To get neutrophils to the inflammation site they must be "captured" by the vascular endothelium, transverse into the tissue, and then migrate to the inflammation site (Sadik et al., 2011). Neutrophils phagocytose pathogens and destroy them in phagocytic vacuoles using protease enzymes housed in granules (Segal, 2005). Due to their destructive nature they can potentially cause tissue damage; therefore, recruitment and activity of neutrophils must be regulated. There are several chemoattractants that have been identified to recruit neutrophils to the site of infection including CXCL8, IL-17, IL-37, leukotriene $\mathrm{B}_{4}\left(\mathrm{LTB}_{4}\right)$, platelet activating factor (PAF), and complement components C5a and C3a (Sadik et al., 2011). Neutrophils are made in the bone marrow and will move out into the periphery in response to the hematopoietic cytokine G-CSF during acute inflammation. PAMPs and DAMPs will activate tissue resident sentinel cells (MФ, mast cells) or stromal cells to release PIMs and neutrophil chemoattractants (chemokines and lipid mediators (LTB 4 and PAF)) (Arancibia et al., 2007; Borregaard, 2010; Williams et al., 2011).

In order for neutrophils to exit the blood stream and migrate to the injury/inflammation site, adhesion molecules must be displayed by the endothelial cells neighboring the area. Neutrophils will engage in a sequence of physical interactions with endothelial cells that can be referred to as the leukocyte adhesion cascade (Williams et al., 2011) and includes rolling, activation, adhesion strengthening, and then diapedesis (Murphy, 2012); events that are orchestrated by $\mathrm{PGE}_{2}$, leukotrienes, adhesion molecules, and chemokines (Calder, 2003; Phillipson et al., 2006; Wegmann et al., 2006; Lou et al., 2007; Borregaard, 2010; Sadik et al., 2011; Murphy, 2012). Lipid mediators such as $\mathrm{LTB}_{4}$ usually act at the start of neutrophil recruitment and at the local site of inflammation as they are rapidly produced but have a short half-life. Chemokines, on the other hand, are produced slower (as they are often regulated transcriptionally and their release is subject to post-translational regulation) and have a longer half-life making them suited to be used later in recruitment cascades and at longer distances (Sidak et al., 2011). In 
tissue, the main job of neutrophils is thought to be phagocytosis; however, in peripheral tissues they can release chemokines, cytokines, and lipid mediators (Scapnin et al., 2000; Kasama et al., 2005;

Borregaard, 2010) contributing to biological mediator production at the inflammation site. While a single neutrophil cannot produce the same quantity of mediators as other immune cells (ex: МФ) due to their sheer number at the inflammation site their mediator production is significant (Kasama et al., 2005; Chou et al., 2010). In producing these mediators, neutrophils can recruit additional neutrophils to the site, aid in orchestrating the progression of an acute inflammatory response from a predominantly neutrophilic infiltrate to a monocytic one (Soehnlein et al., 2009), and participate in the resolution of inflammation through the production of specialized pro-resolving mediators (SPMs) through sequential steps involving endothelial cells and MФs in addition to neutrophils (Serhan and Savill, 2005; Ariel et al., 2006; Borregaard, 2010). When MФs phagocytosis apoptotic neutrophils they decrease their production of IL23 , this leads to a decrease in production of IL-17 from Th17 cells and consequently less G-CSF is produced from fibroblast and endothelial cells in the area to stimulate neutrophil production in the bone marrow (Borregaard, 2010).

Murine Treg cells have been reported to express several TLRs including TLR 4, 5, 7, and 8 (Caramalho et al., 2003) and respond to the TLR4 ligand LPS in vitro. When cultured with LPS, murine Tregs cells proliferated, had increased survival, and increased suppressor function (Caramalho et al., 2003). Activation of Tregs by microbial PAMPs does not stop the immune system from clearing an infection, but it may limit the magnitude of the immune response to protect against host damage and targeting self-antigens (Hori et al., 2002). The balance between effector and regulatory cells depends on the antigens being presented, thus with the onset of acute infection a high amount of nonself peptides are presented resulting in activation of naïve responder cells. As the pathogen is dealt with and the debris is cleared, the predominant type of antigen being presented shifts towards self-antigen being presented to Tregs. Activated Tregs can then work to control inflammation, restore homeostasis, and prevent the activation of naïve, autoreactive $\mathrm{T}$ cells by self-antigen presented in an inflammatory context (Caramalho et al., 2003).

Ligation of PAMPs to TLRs on DCs leads to maturation of the APCs characterized by upregulation or MHCII and the co-stimulatory molecules CD80 and CD86, and the production of cytokines (Murphy, 2012). LPS matures DCs and stimulates them to produce PICs including IL-6, which has been shown to allow activation and function of pathogen specific effector $\mathrm{T}$ cells even in the presence of Tregs cells (IL-6 signaling allows effector T cells to overcome the suppressive effect of Treg cells) (Pasare and Medzhitov, 2003). 
While the response of the innate immune system to a pathogen is not specific to the type of invader (inflammation, phagocytosis, complement), the adaptive immune system tailors its response to the type of pathogen (bacteria, virus, parasite) through subsets of $\mathrm{T}$ helper cells with different functions and through CD8+ T cells and B cells with antigen specific receptors (Murphy, 2012). The different T helper cell subsets are under the control of distinct lineage specifying genes that are activated in response to 1) $\mathrm{CD} 4+\mathrm{TCR}$ recognizing antigen presented by an APC, 2) interaction between co-stimulatory molecules between the T cell and APC, 3) specific cytokines released by the APC (Murphy, 2012). An APC producing IL-12 and IFNץ when presenting antigen to a naïve CD4+ T cell will cause the transcription factors STAT4 and T-bet to become activated leading to a Th1 cell that will produce IL-2 and IFN $\gamma$ while aiding in virus elimination. An APC producing IL-4 and IL-2 will cause the transcription factors STAT6 and GATA3 to be produced by a naïve CD4+ T cell turning it into a Th2 call that will aid in eliminating parasites and produce the cytokines IL-4, IL-5, and IL-10. Naïve CD4+ cells are polarized towards a Th17 phenotype in the presence of IL-17, IL-6, IL-21, and IL-23 from the APC which activate the transcription factors STAT3 and ROR $p t$. Th17 cells are a pro-inflammatory T helper cell subset that produce IL-17 that recruits neutrophils to the site of infection. Treg cells are polarized in response to the cytokines TGF $\beta$ and IL-2 from the APC and the transcription factors STAT5 and Foxp3. Tregs have suppressive functions and produce TGF $\beta$ and IL-10 (Reiner, 2007; Murphy, 2012) Tregs can switch to a Th17 phenotype in the presence of PICs (Koenen et al., 2008), but this switch can be suppressed by IDO (Baban et al., 2009).

\section{Fever}

Fever is an upward resetting of an individual's temperature "set point" (threshold for the activation of thermal responses) in response to infection (Cooper et al., 1964). Recognizing PAMPS, leukocytes release endogenous pyrogens that affect the hypothalamic temperature sensors leading to a rise in the temperature set point. The individual is then in a hypothermic state since body temperature is below the new temperature set point. Effector mechanisms are activated to elevate body temperature including increased metabolic heat production, decreased heat loss by decreasing skin blood flow and evaporative heat loss, and behavioral responses such as seeking an area of warmer temperature (Kluger, 1978).

The major mediator of fever is $\mathrm{PGE}_{2}$ produced by brain vascular endothelial cells (Stitt, 1986; Engström et al., 2012; Wilhelms et al., 2014). The lipid effector is produced in response to pyrogenic cytokines and activates neurotransmitters that raise core body temperature (Evans et al., 2015). Pyrogenic cytokines (IL-1, IL-6, and TNF $\alpha$ ) are produced in response to bacterial products, toxins, or the microbes themselves at the site of infection and travel through the circulation to the brain vascular where they are either actively transported across the blood-brain barrier or through passive transport cross into the brain 
through fenestrated capillaries in the circumventricular organs (Netea et al., 2000; Evans et al., 2015). LPS may also be able to induce fever by stimulating PIC production by endothelial cells in circumventricular organs (Netea et al., 2000). Pathogenic stimuli also cause peripheral $\mathrm{PGE}_{2}$ production by hematopoietic cells that can travel through the blood-brain barrier and initiate a fever response (Netea et al., 2000; Evans et al., 2015).

The cytokines IL-1 and TNF $\alpha$ are produced initially, and both cytokines can then stimulate IL-6 production. All 3 cytokines work to stimulate $\mathrm{PGE}_{2}$ production (Netea et al., 2000; Evans et al., 2015). The systemic or locally produced pyrogenic cytokines act on brain endothelial cells to induce synthesis of the enzymes COX-2 and prostaglandin E synthase 1 resulting in $\mathrm{PGE}_{2}$ production (Engström et al., 2012; Wilhelms et al., 2014). IL-1 receptors mediating COX2 induction, and, thus, $\mathrm{PGE}_{2}$ synthesis, have been identified on brain endothelial cells within the median preoptic nucleus region of the hypothalamus and signal via the p38 MAPK and c-Jun pathway (Konsman et all, 2004; Ching et al., 2007). Il-6 binds to IL6R $\alpha$ on brain endothelial cells inducing COX2 expression and $\mathrm{PGE}_{2}$ synthesis via the STAT3 signaling pathway (Eskilsson et al., 2014). To induce fever, $\mathrm{PGE}_{2}$ binds to the $\mathrm{PGE}_{2}$ receptor 3 (EP3) expressed by thermoregulatory neurons in the median preoptic nucleus region of the hypothalamus (Ushikubi et al., 1998; Lazarus et al., 2007). Neurons expressing EP3 trigger the sympathetic nervous system to release noradrenaline which increases thermogenesis in brown adipose tissue and reduces passive heat loss in extremities by inducing vasoconstriction. Acetylcholine also is released, which increases overall metabolic rate and stimulates muscle myocytes to induce shivering (Hasday et al., 2014; Evans et al., 2015).

In moderation, an elevation in body temperature is beneficial in that it inhibits bacterial growth, increases bactericidal activity of innate immune cells, causes leukocyte mobilization, and stimulates acute protein synthesis, allowing the host to clear the infection and survive (Kluger, 1978; Netea et al., 2000). Specifically, fever-range temperatures increases neutrophil recruitment to local sites of infection and enhances respiratory burst, a bacteriolytic mechanism of neutrophils (Evans et al., 2015). Phagocytic activity of MФs and DCs are increased with heat as well as their response to pathogens through upregulation of TLR2 and TLR4. Heat also increases expression of MHC I, MHC II, and co-stimulatory molecules (CD80 and CD86) by mature DCs and augments secretion of Th1 polarizing cytokines (IL-12, $\mathrm{TNF} \alpha$ ) and CC-chemokine receptor 7 (CCR7) dependent migration through afferent lymphatics to draining lymph nodes; thus increasing their potential to sense pathogens and then present antigen to naïve T cells. 
Several chemokines contain binding sites within their promoter regions for heat-inducible transcription factor heat shock factor protein 1 (HSF1) including chemokines that recruit neutrophils, NK cells, monocytes, CD4+ and CD8+ T cells (CXCL8, CXCL9, CXCL10, CXCL11, and CXCL12) (Hasday et al., 2014), suggesting fever aids in infiltration of immune cells to infection sites. Heat also functions to increase release of immunomodulatory molecules such as cytokines, NO, and heat shock protein 70 (Evans et al., 2015). While febrile temperatures initially increase PIC production by MФs at the inflammatory site, heat also dampens cytokine production after MФs become activated. This is a result of elevated temperature reducing transcription of genes for PICs through repressive activities of the HSF1. In addition, there is reduced recruitment of NF-kB to promoter regions of cytokine encoding genes and reduced cytokine mRNA stability (Evans et al., 2015).

\section{Acute Phase Response}

An acute phase response (APR) is a nonspecific reaction with the goal of restoring homeostasis (Ceciliani et al., 2002; Murata et al., 2004; Gruys et al., 2005; Cray et al., 2009). The APR is part of the innate immune response and can be activated by infection, stress, and inflammation (Murata et al., 2004; Cray et al., 2009). Local response to these triggers is production of PICs that act at the level of the central nervous system (CNS), autonomic nervous system (ANS) and the adrenal gland. PICs acting at the level of the CNS on the HPA axis leads to behavior changes like disinterest in social activities as well as anorexia, adipsia, and lethargy (Tracey, 2002; Gruys et al., 2005; Karrow, 2006). Activation of the ANS can modulate the APR by depressing the release of the cytokines by inflammatory cells and by affecting hypothalamic responses (Karrow, 2006). Cortisol released from the adrenal gland works systemically to cause leukocytosis and alter acute phase protein (APP) production.

Systemic effects that compose the APR include fever, leukocytosis, increased cortisol, decreased thyroxine concentrations, metabolic changes in lipolysis, gluconeogenesis, and muscle catabolism, decreased serum iron and zinc concentrations and changes in APP levels. There can be decreases in the APPs albumin and transferrin (negative APPs), while there can be increases in the APPs C-reactive protein (CRP), serum amyloid A (SAA), haptoglobin (Hp), alpha-1-acid glycoprotein (AGP), ceruloplasmin ( $\mathrm{Cp}$ ), and fibrinogen (positive APPs). APPs are synthesized primarily by hepatocytes when stimulated by the PICs IL-6, IL-1, IFN- $\gamma$, or TNF- $\alpha$ and then released into the bloodstream (Heinrich et al., 1990; Petersen et al., 2004; Murata et al., 2004; Gruys et al., 2005) but also can be made by other tissues including the endometrium (Chapwanya et al., 2013). APPs have various functions such as proteinase inhibitors, enzymes, metal-binding proteins, transport proteins, and coagulation factors. These functions are important in mediating immune function, pathogen trapping, preventing replication of the 
pathogen, tissue repair, and remodeling (Ceciliani et al., 2002; Murata et al., 2004; Petersen et al., 2004; Karrow, 2006; Cray et al., 2009; Thatcher et al., 2010).

There are major, moderate, and minor APPs. Major APPs have low serum concentrations $(<1 \mu \mathrm{g} / \mathrm{L})$ in healthy animals that increase by $100-1000$ fold when activated while moderate APPs increase by 5-10 fold when activated (Eckersall and Bell, 2010). Major APPs tend to show an early and large increase in concentration with a rapid decline while moderate APPs tend to require more time to increase and return to normal values. There can be species specific production and response of APPs. The major APPs in both cows and sheep are haptoglobin (Hp) and serum amyloid A (SAA) (Cray et al., 2009; Lecchi et al., 2012; Chapwanya et al., 2013).

The APR prevents microbial growth while some APPs opsonize microorganisms and activate complement, scavenge cellular remnants and free radicals, neutralize proteolytic enzymes, or modulate the immune response (Gruys et al., 2005). Activities of SAA include being chemotaxic to monocytes, polymorphonuclear cells, and T cells, scavenging potentially dangerous oxidized cholesterol, and opsonization of bacteria (Petersen et al., 2004; Hari-Dass et al., 2005; Cray et al., 2009). Haptoglobin binds free hemoglobin reducing iron availability for bacterial growth and prevents pro-oxidant activity of hemoglobin on tissues (Allison, 1958; Eaton et al., 1982; Petersen et al., 2004; Cray et al., 2009).

Upon activation of the immune system the PICs IL-1, IL-6 and TNF- $\alpha$ are produced. All 3 cytokines have autocrine activities, stimulating their own production. In addition, IL-1 $\beta$ and TNF $\alpha$ stimulate each other and both stimulate IL-6. IL-6 then inhibits the secretion of TNF $\alpha$ and IL-1 $\beta$ ( ; Ceciliani et al., 2002). These cytokines activate the HPA axis as seen by increases in CRH, ACTH, and

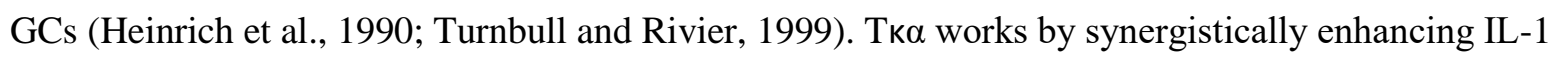
induced ACTH and/or GC secretion (Turnbull and Rivier, 1999), and both amplify and prolong the inflammatory response by activating surrounding cells to release more IL-1 and inflammatory mediators including eicosanoids, NO, and ROS (Tracey, 2002). In addition, IL-1 $\beta$ and IL-6 work together to induce the fever response associated with the APR. GCs inhibit the production of all 3 cytokines to prevent overproduction; however TNF $\alpha$ has the greatest sensitivity to this suppression while IL-6 is fairly resistant to this suppression. IL-6 acts synergistically with GCs to stimulate hepatic APP production (Heinrich et al., 1990; O’Conner et al., 2000; Ceciliani et al., 2002; Murata et al., 2004) and to down regulate cytokine production by monocytes and MФs (Baybutt and Holsboer, 1990; Ceciliani et al., 2002; Murata et al., 2004).

The APR involves activation of the HPA axis and production of GCs which can have antiinflammatory effects through regulation of gene transcription. Binding to intracellular steroid receptors, 
the ligand-receptor complex migrates into the nucleus to bind to specific DNA sequences that oppose the function of the transcription factors AP-1 and NF- $\kappa$ B. This will prevent transcription of pro-inflammatory genes such as TNF- $\alpha$, pro-IL-1 $\beta$, and IL-6. Reduced TNF- $\alpha$ and IL-1 $\beta$ affects normal downstream actions of these cytokines such as expression of the enzymes COX2 and iNOS as well as expression of intracellular adhesion molecule-1 (ICAM-1). GCs also suppress expression of IFN $\gamma$ and inflammatory genes for cytokines involved in $\mathrm{T}$ cell growth and T helper cell polarization (IL-2, IL-4, IL-15, IL-17). While decreasing expression of PICs, GCs increase expression of anti-inflammatory molecules, like IL-10 and the IL-1 type 2 decoy receptor (Dinarello, 2010).

Acute phase proteins are considered a nonspecific diagnostic tool; while measurements cannot be used as a marker for a particular disease, APP levels provide evidence that an individual has subclinical inflammation or an infection and can be used in prognosis and in monitoring response to therapy (Petersen et al., 2004; Gruys et al., 2005; Eckersall and Bell, 2010). APPs have been used as biomarkers for monitoring inflammation, infection, and trauma in humans for decades (Eckersall and Bell, 2010) and in recent years have progressively been used as biomarkers in veterinary medicine in companion and farm animals (Petersen et al., 2004; Murata et al., 2004; Cray et al., 2009; Eckersall and Bell, 2010).

\section{Inflammasome}

Due to its highly pro-inflammatory nature and potential to damage host cells extra precaution is taken when activating IL-1 $\beta$. In order for IL-1 $\beta$ to be synthesized, processed to its active form and secreted by МФ, the cell must respond to two distinct stimuli and an inflammasome must form (Meylan et al., 2006; Sutterwala et al., 2006). NOD-like receptors (NLRs) are a family of PRRs that are mostly expressed in the cytosol; NALP3 is a member of the NLR subfamily NALP (Martinon et al., 2009). Upon activation, the NALP3 inflammasome forms from a combination of the NALP3 protein, CARDINAL., ASC (apoptosis-associated speck-like protein containing a CARD (caspase recruitment domain)), and caspase-1. The NALP3 protein (also known as cryopyrin) is composed of an N-terminal pyrin domain (effector domain), carboxy-terminal ligand-sensing leucine rich repeats (LRRs), the central nucleotide domain NACHT (nucleotide-binding and oligomerization domain) (mediates oligomerization), and a NACHT-associated domain (NAD) (Meylan et al., 2006; Martinon et al., 2009). The adaptor molecule, ASC controls the activation of caspase-1 which cleaves two members of the IL-1 family of ligands, proIL-1 $\beta$ and pro-IL-18 into their biologically active, secreted forms (Meylan et al., 2006; Sutterwala et al., 2006; Dinarello, 2007; Dinarello, 2009; Schroder and Tschopp, 2010). Caspases are cysteine proteases that initiate or execute functions involved in inflammation or cell death, therefore, their function is tightly controlled by being synthesized as inactive zymogens that have to be activated by proteolytic cleavage (Martinon et al., 2009; Schroder and Tschopp, 2010) 
The first signal needed to get IL-1 $\beta$ produced is an inflammatory signal recognized by a TLR, such as LPS binding to TLR4. Downstream of the TLR, NF-kB will be activated leading to transcription of the IL-1 $\beta$ gene, which is then translated into an immature pro-form of the cytokine (Meylan et al., 2006; Sutterwala et al., 2006). The second necessary signal is one that will activate the inflammasome to form allowing caspase- 1 to cleave the cytokine into its mature form which can then be secreted. The NALP3 inflammasome responds to some PAMPs such as peptidoglycan and its degradation products, bacterial RNA, and small antiviral compounds (Martinon et al., 2004; Kanneganti et al., 2006; Schroder and Tschopp, 2010). The NALP3 inflammasome also responds and becomes activated in response to DAMPs that cause $\mathrm{K}^{+}$efflux resulting in low intracellular concentrations of potassium (Perregaux and Gabel, 1994; Meylan et al., 2006; Mariathasan et al., 2006; Pétrilli et al., 2007; Schroder and Tschopp, 2010). This occurs when extracellular ATP binds the $\mathrm{P}_{2} \mathrm{X}_{7}$ receptor or in response to $\mathrm{NAD}^{+}$, bacterial toxins, or uric acid crystals (Perregaux and Gabel, 1994; Solle et al., 2001; Shi et al., 2003; Kahlenberg and Dubyak, 2004; Martinon et al., 2006; Kanneganti et al., 2006; Kawamura et al., 2006).

Lipopolysaccharide does not activate the NALP3 inflammasome (Martinon et al., 2004; Mariathasan et al., 2006), however, in combination with DAMPs that do, LPS leads to the production of mature IL-1 $\beta$ (Meylan et al., 2006; Mariathasan et al., 2006; Sutterwala et al., 2006). TLRs agonists (ex: LPS) induce pro-cytokine synthesis and DAMPS (ex: extracellular ATP) stimulate caspase-1-dependent cleavage and secretion of the active form of IL-1 $\beta$ and IL-18 (Mariathasan et al., 2006; Sutterwala et al., 2006). ATP induced $\mathrm{K}^{+}$efflux through $\mathrm{P} 2 \mathrm{X}_{7}$ signaling causing an increase in intracellular $\mathrm{Ca}^{2+}$ may also be necessary for activation of active phospholipase $\mathrm{C}$ and calcium dependent PLA2 needed for exocytosis of IL-1 $\beta$ containing lysosomes (Andrei et al., 2004). Phospholipases act on phospholipids in membranes to produce lipid metabolites that are involved in the final stages of membrane fusion (Goñi and Alonso, 2000; Brown et al., 2003).

It was suggested that TLR signaling might also be required for expression of some of the inflammasome components, since although ATP was required for caspase-1 activation, ATP by itself without LPS priming did not activate caspase 1 (Mariathasan et al., 2006; Sutterwala et al., 2006). Indeed, Bauernfeind et al (2009) reported that NF-kB activation by TLR agonists or PICs (TNF $\alpha$ ) is required for gene transcription of Nlrp3. The necessity of an extracellular signal that leads to NF-kB activation to get transcription of NLRP3, pro-IL-18 and pro-IL-18 combined with a host danger signal to activate NLRP3 allowing for inflammasome assembly may provide insurance that IL-1 $\beta$ and IL-18 are produced when pathogenic bacteria are present and not in response to commensal bacteria (Meylan et al., 2006; Bauernfeind et al., 2009). Caspases also have functions related to forms of cell death. At low levels of 
caspase- 1 activation, the protease functions to stimulate cell survival responses and control intracellular growth in addition to forming active PICs. However, if activation of caspase-1 crosses a threshold level the MФ undergoes pyroptosis, a form of cell death characterized by rapid plasma-membrane rupture and release of proinflammatory intracellular contents (Bergsbaken et al., 2009). With this form of cell death caspase- 1 is responsible for the formation of pores in the plasma membrane that results in loss of control of cellular ionic gradients, a net increase in osmotic pressure, water influx that causes the cell to swell and ultimately osmotic lysis that releases inflammatory intracellular mediators (Fink and Cookson, 2006; Bergsbaken et al., 2009). Neutrophils are able to process the IL-1 $\beta$ precursor independent of caspase-1 using other enzymes. In these cells, proteinase-3 can cleave pro-IL-1 $\beta$ into its active form (Guma et al., 2009; Joosten et al., 2009).

Primary sources of IL-1 $\beta$ are innate immune cells including tissue MФs, blood monocytes, and DCs, however, NK and B lymphocytes can also produce the cytokine (Dinarello, 2009). IL-1 $\beta$ induces gene expression and synthesis of COX2, PLA2, and iNOS resulting in production of $\mathrm{PGE}_{2}, \mathrm{NO}$, and platelet activating factor (PAF). These mediators are involved in fever, lowered pain threshold, vasodilation, and hypotension (Dinarello, 2009). IL-1 $\beta$ also induces the expression of adhesion molecules by mesenchymal and endothelial cells to aid in the infiltration of immune cells into the inflammatory area (Dinarello, 2009). Cytokines exist in "cascades" and tend to act in a synergistic fashion (Dinarello, 2010). In general, PICs will function to induce other PICs, feedback to induce each other and stimulate further expression of themselves to increase inflammatory cytokine expression. IL-1 $\beta$ will stimulate production of other cytokines (IL-6, IL-17) and APPs (Dinarello, 2009). IL-1 $\beta$ is involved in IL-17 production by stimulating production of IL-6 and IL-23; Th17 polarizing cytokines that activate STAT3 which then induces the Th17 transcription factor RORyt (Dinarello, 2009; Murphy, 2012). IL-17 is chemoattractant to neutrophils and MФs and has also been shown to stimulate the production and release of IL-1 $\beta$ from primary human blood monocytes (Dinarello, 2009; Dinarello, 2010; Murphy, 2012).

Inflammasomes are also responsible for cleavage of pro-IL-18 into its mature form, another PIC. IL-18 in conjunction with IL-12 induces IFN $\gamma$ production by other immune cells and naïve T cells to polarize towards a Th1 phenotype. With regards to IFN $\gamma$ production, IL-18 and IL-12 signaling leads to activation of transcription factors that induce transcription of IFN $\gamma$. Working through a signaling pathway with similar components to TLR signaling, IL-18 activates MyD88, IRAK (IL-1 receptor-associated kinase) proteins, and TRAF6 (TNF receptor-associated factor) to activate p38 MAP, AP-1 and cause translocation of NF-kB into the nucleus. IL-12 leads to activation of STAT4 and AP-1 (Robinson et al., 1997; Nakanishi et al., 2001). These transcription factors bind the promoter region of IFN $\gamma$ and together cause gene transcription (Nakanishi et al., 2001). Th1 (but not Th2) cells express IL-18R $\alpha$ (Robinson et 
al., 1997; Yoshimoto et al., 1998; Xu et al., 1998) and IL-18 induces cell proliferation of Th1 cells in addition to acting as a costimulant molecule to augment production of IL-2, IL-2R $\alpha$, and GM-CSF production by these cells (Robinson et al., 1997; Yoshimoto et al., 1998). IL-18 (along with IL-2) in the absence of IL-12 cause naïve T cells to polarize towards a Th2 phenotype and production of IL-4 and IL13 (Nakanishi et al., 2001).

Because IL-1 and IL-18 have such potential to cause host damage with their inflammatory nature, not only is their production/activation tightly regulated but there are specific mechanisms to control their function once they have been secreted to prevent runaway inflammation. IL-1Ra is a receptor antagonist that binds the IL-1 receptor with greater affinity than IL-1, thus reducing IL-1 $\beta$ function. IL-18 binding protein (IL-18BP) is an endogenous neutralizer of IL-18 activity as it has greater affinity for IL-18 than the IL-18 receptor has for the cytokine (Novick et al., 1999; Dinarello, 2009).

\section{Lipopolysaccharide}

Lipopolysaccharide (LPS) is a component of gram negative bacteria cell walls and is a PAMP for host immune cells to recognize pathogens. LPS will directly activate monocytes/MФs to produce PICs (TNF- $\alpha$, IL-1 $\beta$, IL-6, IL-8), eicosanoids (PGE 2 ), NO, and MMPs (Calder, 2003). LPS will induce the expression of adhesion molecules (ICAM-1, VCAM-1, E-selectin) by endothelial cells and leukocytes which are needed for the movement of cells into the inflammatory/infected site (Calder, 2003). The cytokines and eicosanoids produced will regulate the whole-body response to infection/injury (fever, pain sensitivity) (Calder, 2001). While infections need to be cleared to protect the host, over activation of the PRR pathways and production in PICs is detrimental to pregnancy. Neutralization of LPS or blockage of TLR4 signaling has been shown to prevent fetal loss in murine pregnancy (Friable et al., 2011).

Endotoxins can decrease embryo cleavage rates, blastocyst formation and impair implantation, resulting in embryonic loss (Deb et al., 2004). LPS mediates its biological effects mostly through PICs. While inflammatory cytokines and growth factors play a role in blastocyst development and implantation, the quantity and timing of their expression must be tightly controlled, any disturbance in the level of expression at the feto-maternal interface can cause embryonic loss (Deb et al., 2004; Agrawal et al., 2013a). Cell adhesion molecules, cytokines, growth factors, endometrial epithelial integrins, MMPs, PGs, and COX-2 are important for successful implantation playing roles in tissue remolding and regulation of trophoblast invasion. Expression of these molecules by uterine and trophoblast cells is regulated by cytokines and growth factors (IGF, TGF, IL-1, IL-6, TNF) (Lim et al.,, 1999; Bischof et al., 2000; Dey et al., 2004). 
The antifertility effects of LPS can be attributed to modulation of the synthesis and secretion of cytokines and growth factors at the feto-maternal interface altering preimplantation embryonic growth and development along with appropriate inflammatory reactions at the implantation site. Epithelial and stromal cells have been shown to respond to LPS in vitro by phosphorylation of MAPK and nuclear translocation of NF-kB, secretion of $\mathrm{PGF}_{2 \alpha}, \mathrm{PGE}_{2}$, IL-6, CXCL8, and increased expression of antimicrobial peptides (MUC1, lingual antimicrobial peptide, tracheal antimicrobial peptide) (Herath et al., 2006; Davies et al., 2008; Cronin et al., 2012). Changing the levels and timing of production of implantation relevant cytokines can lead to preimplantation embryonic loss or implantation failure (Deb et al., 2004). For example, IL-1 $\alpha$ and $\beta$ binding to their receptors on the uterine endometrium trigger integrin expression during implantation (Simón et al., 1998) and IL-1 stimulates production of endometrial LIF, which is important for implantation (Dimitriadis et al., 2005; Agrawal et al., 2013a). Altered production of cytokines can accelerate or delay the uterine transition to the receptive state disrupting coordination between embryonic and uterine development. This can mean a receptive uterus and an implantation competent blastocyst do not occur at the same time. In addition to altering uterine receptivity, LPS effects can make the developing blastocyst incompetent for implantation (Deb et al., 2004). Endotoxin is known to induce embryonic death (Giri et al., 1990) and has been shown to increase DNA damage in preimplantation embryos associated with a decrease in heat shock proteins (HSP 90, HSP70, HSP60), decrease embryo cleavage rate, decrease blastocyst formation, and induce degeneration, fragmentation, and developmental arrest of the preimplantation embryo (Dumoulin et al., 1991; Randall et al., 1991; Dostál et al., 1996; Jaiswal et al., 2006; Jaiswal et al., 2013).

Mediated by PICs, LPS can inhibit steroidogenesis, increase PG production, and cause luteolysis of the CL (Bagavandoss et al., 1990; Gorospe et al., 1992; Telleria et al., 1998). PG production can result in embryonic death by acting as an abortifacient and/or causing luteolysis of the CL and loss of $\mathrm{P}_{4}$ production (Deb et al., 2004). Among other actions, $\mathrm{P}_{4}$ regulates uterine receptivity for blastocyst attachment and coordinates uterine-embryo interactions (Paria et al., 2002). In the mouse, LPS (given on day 0.5 p.c. ) decreased serum $\mathrm{P}_{4}$ while increasing serum $\mathrm{E}_{2}$ and this higher $\mathrm{E}_{2} / \mathrm{P}_{4}$ ratio during preimplantation led to unsuccessful pregnancy. The change is steroid levels may have prevented the uterus from becoming receptive or prevented the blastocysts from developing to the stage where they could implant (Agrawal et al., 2011) In addition, LPS given to mice was shown to alter the mRNA expression of both the $\mathrm{P}_{4}$ and $\mathrm{E}_{2}$ receptor in embryos and the uterus on preimplantation days which will affect the ability of the hormones to regulate implantation (Agrawal et al., 2013b). E. Coli endotoxin infusions ( 1.0 or $2.5 \mu \mathrm{g} / \mathrm{kg}$ over 6 hours) to first trimester cows caused an increase in $\mathrm{PGF}_{2 \alpha}$ followed by a drop in $\mathrm{P}_{4}$ and pregnancy loss (Giri et al., 1990). 


\section{Bacterial Infections during Pregnancy}

The female reproductive tract has several anatomical barriers to block ascending microbes from reaching the uterus including the vulva, vagina, cervix, the stratified squamous epithelium of the vagina, and the columnar epithelium of the endometrium. As a second line of defense, the mucosa of the reproductive tract contains antimicrobial peptides and mucosal glycoproteins to neutralize bacteria and prevent them from reaching the epithelium and causing infection. The bovine endometrium contains the antimicrobial peptides $\beta$-defensins, lingual antimicrobial peptide, and tracheal anti-microbial peptide (Davies et al., 2008; Chapwanya et al., 2013). Gene expression for APPs has also been found in the uterus and may be involved in protection against infection (Lecchi et al., 2012; Chapwanya et al., 2013), however, the major producer of APPs is the liver in response to PICs. The female reproductive tract also contains the complement system which is involved in opsonization of infected cells to mark them for phagocytosis and killing of infected cells (Sheldon et al., 2014). If microbes surpass all the passive defense systems in the reproductive tract the result is infection.

Apoptosis inducing cytokines, such as TNF $\alpha$ and IFN $\gamma$ can cause pre-implantation embryonic loss or impair embryo development. TNFa has been seen in vitro to increase the percentage of apoptotic mouse blastomeres (Fabian et al., 2007) and IFNץ inhibits the embryotrophic factor GM-CSF (Robertson et al., 1992b). GM-CSF is produced by epithelial cells lining the oviduct and uterus around the time of conception in response to seminal fluid and is required for normal blastocyst development and fetal viability (Robertson, 2007; Sharkey et al., 2007). GM-CSF in the mouse has been reported to promote blastocyst formation and increase the number of viable blastomeres by inhibiting apoptosis and the stress response and facilitating glucose uptake (Robertson et al., 2001; Chin et al., 2009). With regards to generating fetal tolerance, GM-CSF plays a role in upregulating MHC-II and the co-stimulatory molecules CD80 and CD86 on APCs like DCs and macrophages and a deficiency in the cytokine impairs Ag presentation and T cell response to Ag (Moldenhauer et al., 2010). Paternal antigen must be processed and bound to an MHC II molecule to be presented to T cells by APCs and the co-stimulatory molecules are critical for stabilizing the peptide-MHC-TCR interaction to generate strong Ag-specific $\mathrm{T}$ cell responses (Murphy, 2012). Inadequate expression of these molecules by APCs in the reproductive tract after coitus will negatively impact Treg cell generation specific to paternal antigens and fetal tolerance. Therefore, a uterine infection around the time of conception is likely to prevent a successful pregnancy.

\section{Omega-3 Polyunsaturated Fatty Acids}

Omega-3 poly-unsaturated fatty acids (PUFA) are known to have anti-inflammatory effects for several reasons. They can effect eicosanoid production by displacing AA in cellular membranes (Jaudszus et al., 2013) reducing the availability of substrate for production of inflammatory PGs and LTs or by 
competing with AA for the COX and lipoxygenase (LOX) enzymes. Alterations in the fatty acid composition of membranes can also modify membrane fluidity, lipid raft formation, and cell signaling. Inflammation is associated with LTs, PGs, and polymorphonuclear neutrophil (PMN) recruitment to the inflammatory site. Resolution of inflammation is associated with lipoxin (LX) biosynthesis and decreased PMN infiltration (Levy et al., 2001). During inflammation, AA can go through the COX pathway to produce $\mathrm{PGs}$ or the LOX pathway to produce $\mathrm{LTB}_{4}$. $\mathrm{LTB}_{4}$ increases vascular permeability, is a vasoconstrictor, enhances local blood flow, is chemoattractant to leukocytes, induces release of lysosomal enzymes, enhances ROS generation and production of PIC (TNF $\alpha$, IL-1 $\beta$, IL-6). (Calder, 2003; Afonso et al., 2012).

Omega-3 FAs inhibit AA metabolism by COX1 and shift the vascular balance to higher levels of $\mathrm{PGI}_{2}$ by coupling endothelial COX2 with PGI synthase (PGIS) (Norris and Dennis, 2012; Dennis and Norris, 2015). Possible physiological responses of $\mathrm{PGI}_{2}$ binding to the IP receptor include decreased platelet aggregation, hyperalgesia, vasodilation, increased IL-10 levels, and decreased TNF $\alpha$ levels (Shinomiya et al., 2001; Dennis and Norris, 2015). Omega-3 PUFAs can also shunt AA from the inhibited COX pathway into the 5-LOX pathway which produces LTs (involved in inflammation) as well as lipoxins, resolvins, protectins (resolve inflammation) (Dennis and Norris, 2015). Omega-3 PUFAs also modulate inflammation independent of eicosanoid production by altering gene expression of PICs and adhesion molecules (on endothelial cells and leukocytes) (Calder, 2003; 2011). Omega-3 PUFAs can be made into eicosanoids by the PTGS and LOX enzymes but these molecules have anti-inflammatory effects and have been termed specialized pro-resolving mediators (SPMs) (Bannenberg and Serhan, 2010; Serhan and Petasis, 2011). Cytoplasmic phospholipase A (cPLA $)$ acts on phospholipids to release free PUFA, including AA, EPA, and DHA. A diet with high amounts of $\omega$-3 PUFAs will result in cellular membranes with more $\omega$-3 PUFAs and less AA which will affect the PUFA released by $\mathrm{CPA}_{2}$ and the subsequent eicosanoids produced, whether pro-inflammatory PGs and LTs or anti-inflammatory SPMs.

\section{Resolution of Inflammation and Specialized Pro-Resolving Mediators}

Specialized pro-resolving mediators (SPMs) (di- and trihydroxylated metabolites of long chain PUFAs) are eicosanoids and docosanoids produced by LOX, COX, and cytochrome P450 enzymes working alone or in combination. SPMs include LXs (metabolites of the $\omega-6$ FA AA), resolvins (Resolvin E series: COX2 derived metabolites of the $\omega$-3 FA, EPA; Resolvin D series: 15-LOX derived metabolites of the $\omega-3$, DHA; Resolvin $\mathrm{D}_{\mathrm{n}-3 \mathrm{DPA}}$ : 15-LOX derived metabolite of the $\omega-3$ FA, DPA; Resolvin T: COX2 derived metabolite of the $\omega-3$, DPA), protectins (15-LOX derived metabolites of the $\omega-3$, DHA), and maresins (MФ mediators in resolving in inflammation; MaR1 and MaR2 derived from DHA) (Serhan, 2007; Serhan et al., 2014;Weylandt, 2016). 
Special pro-resolving mediators are produced at specific temporal intervals to resolve inflammation and aid in the return to a state of homeostasis (Bannenberg and Serhan, 2010). SPMs accelerate bacterial clearance (Chiang et al., 2012), promote recruitment and activation of monocytes, promote neutrophil clearance (efferocytosis: "phagocytic clearance of apoptotic cells before they undergo secondary necrosis"), inhibit neutrophil diapedesis, inhibit PIC expression, and turn on anti-inflammatory cytokine expression (Schwab et al., 2007; Serhan and Petasis, 2011; Colas et al., 2014; Serhan, 2014; Serhan et al., 2014; Dennis and Norris, 2015). The process of efferocytosis increases SPM biosynthesis by PMN, including the resolvins D2, and E2 (Norling et al., 2011). Apoptotic PMNs phagocytosed by MФs induce generation of SPMs (LXA $, \mathrm{RvE} 1, \mathrm{PD} 1)$ by the MФ which further increases phagocytic activity and regulates chemokine/cytokine production (个IL-10, $\downarrow$ IL-6 and IFNץ) (Schwab et al., 2007). Maresin $1(\mathrm{MaR} 1)$, is produced by MФs when its biosynthetic pathway is activated during phagocytosis, it functions to reduce neutrophil migration and increase phagocytosis by MФs (Serhan, 2007; Serhan and Petasis, 2011). MФs with the M2 phenotype produce the SPMs MaR1 and lipoxin $\mathrm{A}_{4}\left(\mathrm{LXA}_{4}\right)$ and lower amounts of $\mathrm{LTB}_{4}$ and PGs compared to M1 MФs (Serhan and Chiang, 2013), fitting with M2 MФ's role of tissue repair and inflammation resolution.

The transiently and temporal specific production of SPMs is brought about by the activation of the biosynthetic pathways to produce SPMs through interaction of inflammatory leukocytes with endothelial cells, epithelial cells, and MФs at the inflammation site (Bannenberg and Serhan, 2010). The switch in production of LT to LX (pro-inflammatory to resolution) is mediated by first-phase eicosanoids. $\mathrm{PGE}_{2}$ and $\mathrm{LTB}_{4}$ are produced during inflammation, $\mathrm{LTB}_{4}$ recruits neutrophils to the inflammatory site whereas $\mathrm{PGE}_{2}$ sets up the resolution phase by amplifying LX expression. $\mathrm{PGE}_{2}$ (and $\mathrm{PGD}_{2}$ ) accomplishes this by increasing gene expression for 15-LOX (which produces $\mathrm{LXA}_{4}$ ) through a mechanism that increases intracellular cAMP, initiating RNA processing of the 15-LOX transcript in PMNs, and inhibiting expression of 5-LOX (Levy et al., 2001). As neutrophils migrate into the tissue they will encounter $\mathrm{PGE}_{2}$ that initiates phenotypic changes by regulating gene expression (Levy et al., 2001). Once 15-LOX is transcribed, activity may need to be induced by secondary exposure. Once active, neutrophils will produce LXs instead of LTs from AA and resolution will be initiated (Levy et al., 2001).

Lipoxin $\mathrm{A}_{4}$ has been shown to inhibit neutrophil chemotaxis but enhance M $\Phi$ chemotaxis and stimulate MФ phagocytosis of apoptotic neutrophils (Lee et al., 1989; Mitchell et al., 2002). LXA 4 has also been shown to increase IL-10 which then inhibits TNF $\alpha$ (Souza et al., 2007). Both LTs and LXs are produced from AA in the LOX pathway. The PMN chemokine, $\mathrm{LTB}_{4}$, is produced from the 5-LOX pathway and also influences release of neutrophil granule products and superoxide anions (Borgeat and 
Naccache, 1990). Lipoxins are a separate class of LOX generated eicosanoids that oppose the functions of LTs by working in an autocrine manner to regulate leukocyte function (Levy et al., 2001).

Resolvins are produced and act locally by binding to G-protein coupled receptors to elicit their biological actions in the picogram-nanogram range (Serhan and Petasis, 2011; Chiang et al., 2012). Resolvin E1 (RvE1) binds to the ChemR23 receptor on monocytes and DCs reducing DC migration, decreasing IL-12 production, and enhancing macrophage phagocytosis (Arita et al., 2005; Ohira et al., 2010). RvE1 also interacts with the BLT1 receptor. BLT1 is also the receptor for $\mathrm{LTB}_{4}$, signaling neutrophil survival, chemotaxis of neutrophils, and NF-kB activation. RvE1 blocks $\mathrm{LTB}_{4}$ from binding to the BLT1 receptor, thus stopping neutrophil infiltration and attenuating NF-kB activation. RvE1-BLT1 interaction also promotes apoptosis of PMNs and clearance of apoptotic cells by macrophages (Arita et al., 2007; El Kebir et al., 2012).

Special pro-resolving mediators also function through control of microRNAs to change leukocyte activity (Li et al., 2013). Binding to GPR32 ALX/FPR2, resolvin D1 (RvD1) upregulates miR-208a which increases IL-10 production. RvD1 also upregulates miR-219 causing decreased 5-LOX expression and thus decreased production of $\mathrm{LTB}_{4}$ while increasing 15-LOX expression and production of protectin D1 (Recchiuti et al., 2011; Krishnamoorthy et al., 2012; Fredman et al., 2012).

Resolvins and protectins have been seen to control the magnitude and duration of inflammation in animal disease models (Schwab et al., 2007), to regulate inflammatory pain (Xu et al., 2010) and to increase animal survival (Serhan and Chiang, 2013). Resolution of an E. Coli infection in mice was associated with elevated levels of host protectin D1, resolvin D5 and D1 that enhanced bacterial killing and SPMs given to mice decreased the amount of antibiotics needed for microbial clearance (Chiang et al., 2012). Exogenous $\mathrm{LXA}_{4}$ was reported to increase survival in a sepsis rat model by decreasing levels of PIMs through reduction of NF-kB phosphorylation in MФs. In addition, $\mathrm{LXA}_{4}$ decreased blood bacterial load by increasing recruitment of MФs to phagocytosis the bacteria (Walker et al., 2011). LXs have also been reported to stimulate the production of bactericidal peptides by mucosal epithelia (Canny et al., 2002). Human Th2 cells have been reported to produce protectin D1 that reduces T cell migration, promotes T cell apoptosis, and decreases TNF $\alpha$ and IFNץ (Ariel et al., 2005). Exogenous protectin D1, along with RvE1 and LXA4 upregulate CCR5 expression on leukocytes that bind chemokines and facilitate leukocyte clearance and resolution of inflammation (Ariel et al., 2006). Dietary omega-3 PUFA in humans has been shown to be associated with increased plasma SPMs and Colas et al (2014) also reported increased phagocytosis of E. Coli in whole blood collected from volunteers that had taken capsules containing essential fatty acids (Mas et al., 2012; Colas et al., 2014). 
The role of RvD1 in inflammation resolution was examined in a LPS-induced acute lung injury murine model (Wang et al., 2011; Liao et al., 2012). Pretreating mice with RvD1 before LPS increased survival, inhibited LPS induced recruitment of PMNs and mononuclear leukocytes and IL-6 and TNFa production in bronchoalveolar lavage fluids. RvD1 reduced the LPS-increase in COX-2, iNOS, NO, $\mathrm{PGE}_{2}$, and adhesion molecules. These affects are thought to be mediated at least in part through RvD1 interacting with the LXA4 receptor and inhibiting the MAPK and NF-kB pathways (Wang et al., 2011). Liao et al (2012) also reported reduced leukocyte counts, TNF $\alpha$ and IL-6 in bronchoalveolar lavage fluids of mice treated with RvD1 before LPS. Based on staining they also reported reduced LPS-induced lung inflammation with RvD1. Their conclusion was RvD1 works at least in part by suppressing IkB $\alpha$ degradation and NF-kB nuclear translocation by a pathway partly dependent on PPAR $\gamma$ activation (Liao et al., 2012), although RvD1 has not been shown to directly interact with PPAR $\gamma$ (Krishnamoorthy et al., 2010)

Resolvin D2 (RvD2) promotes inflammation resolution by limiting PMN infiltration, enhancing phagocytosis of bacteria, and stimulating efferocytosis through binding of the GPR18 receptor (Chiang et al., 2015). Spite et al (2009) studied the role of RvD2 in inflammation resolution during microbial infection. They concluded that RvD2 reduces PMN infiltration to the inflammation site by affecting leukocyte-endothelial interactions by reducing platelet activating factor induced surface expression of adhesion molecules (L-selectin and integrin beta 2) by PMNs (Spite et al., 2009). In addition, RvD2 stimulates local NO production at levels that have anti-adhesive effects instead of pro-inflammatory effects (Kubes et al., 1991; Spite et al., 2009) RvD2 also reduced complement (C5a) mediated PMNendothelial interactions and C5a stimulated extracellular superoxide generation. When they examined the effects of RvD2 in a murine sepsis model using cecal ligation and puncture they reported RvD2 reduced the amount of live aerobic bacteria in the blood and peritoneum 12 hours after the procedure. The amount of leukocytes and specifically PMNs that infiltrated into the peritoneum was significantly reduced. RvD2 promoted phagocyte-dependent bacterial clearance and in vitro was seen to enhance $М \Phi$ phagocytosis. While enhancing bacterial clearance, RvD2 also reduced PICs (IL-6, IL-1 $\beta$, IL-23, IL-17, TNF- $\alpha$ ), the chemokine CXCL2 (secreted by monocytes and MФ, recruits polymorphonuclear leukocytes), and PIMs $\left(\mathrm{PGE}_{2}\right.$ and $\left.\mathrm{LTB}_{4}\right)$ (Spite et al., 2009). Lastly, this group reported that when human PMNs were preincubated with RvD2 before incubation with E. coli, the neutrophils had enhanced E. coli phagocytosis that was associated with an increase in intracellular ROS (Spite et al., 2009).

Docosapentaenoic acid (DPA) is a 22 carbon PUFA with 5 double bonds and exists as two isomers, a $\omega-3$ (22:5n-3) and as a $\omega-6$ (22:5n-6) (Weylandt, 2016). The body can turn alpha-linolenic acid (ALA) (18:3 n-3) into EPA. EPA and AA can be converted into DPA; elongation of EPA (20:5n-3) will 
give the $\omega$-3 isomer of DPA (22:5n-3) while elongation followed by desaturation of AA (20:4n-6) will give the $\omega-6$ isomer of DPA (22:5 n-6). Elongation of EPA (20:5n-3) followed by desaturation provides DHA (22:6n-3). (Dalli et al., 2013). Derivatives of both DPA isomers have been reported to have proresolving effects with regards to inflammation (Weylandt, 2016).

With regards to the $\omega-6$ isomer, using the MФ cell line RAW 265.7, the DPA derivatives (17S)hydroxy-docosapentaenoic acid (17-HDPAn-6) and (10,17S)-dihydroxy-docosapentaenoic acid (10,17HDPAn-6) and the DHA derivative 17(R/S)-hydroxy-docosahexaenoic acid (17-HDHA) were reported to increase phagocytosis by the MФ, decrease gene expression of TNF $\alpha$ and inducible NO synthase while increasing expression of the IL-1ra, suggesting polarization of MФ towards a M2 phenotype and inflammation resolution (Chiu et al., 2012). Derivatives of the $\omega-3$ isomer of DPA include protectin D1, the $\mathrm{D}$ series resolvins (RvD) and the maresins (MaR). Mice given an intraperitoneal injection of zymosan (binds TLR2) after an intravenous injection of n-3 DPA derived products had reduced neutrophil recruitment, decreased IL-6 and decreased MCP-1 levels (measured in exudates) compared to mice receiving only zymosan (Dalli et al., 2013). In vitro studies showed that human neutrophils incubated with n-3 DPA products had reduced adhesion to TNF $\alpha$ activated endothelial cells and reduced chemotaxis towards an CXCL8 gradient while incubation of human MФ with n-3 DPA products before addition of zymosan increased phagocytosis (Dalli et al., 2013).

The newly discovered 13-series DPA n-3 resolvins (RvT1, RvT2, RvT3, RvT4) also have antiinflammatory activities. They are produced by transcellular biosynthesis, where COX2 in endothelial cells produces the intermediate 13-HDPA which is then converted to RvT1, RvT2, RvT3, and RvT4 by lipoxygenation in neutrophils (Dalli et al., 2015). RvTs increased phagocytosis of E. coli, efferocytosis of apoptotic neutrophils and the production of ROS by human MФs in vitro. Human Neutrophils exposed to RvT2 also had increased phagocytosis of E. coli and produced more ROS. RvTs blocked components of the inflammasome in MФs, decreasing caspase-1 and IL-1 $\beta$ expression (Dalli et al., 2015). Administration of RvTs 2 hours after intraperitoneal E. coli inoculation in mice dose-dependently increased survival rates. Administration of RvTs right before inoculation protected mice from hypothermia, limited neutrophil recruitment to the inflammation site, increased bacterial phagocytosis, reduced MФ inflammasome activity (as seen be reduced expression of caspase- 1 and IL- $1 \beta$ levels), and reduced systemic $\mathrm{PGD}_{2}$ and $\mathrm{PGE}_{2}$ levels (Dalli et al., 2015). The reduced PGs could be due to RvTs regulating inflammasome activity, as some types of inflammasomes have been reported to produce eicosanoids in response to TLR activation (von Moltke et al., 2012). 
Jones et al (2013a) reported that feeding pregnant rats a diet supplemented with $\omega-3$ PUFAs increased maternal plasma $\omega-3$ PUFAs, increased placental $\omega$-3 PUFAs, reduced placental oxidative stress, and increased placental and fetal growth. Supplementation with $\omega-3$ PUFAs also resulted in increased levels of $L O X 15 \mathrm{~b}$ expression (enzyme driving initial step in resolvin and protectin formation) LOX5 expression (enzyme for final stages of resolvin production), resolvins, protectins, and their precursors in the labyrinth zone of the rat placenta (Jones et al., 2013b), demonstrating that the placenta is capable of producing SPMs and production can be increased with dietary $\omega$-3 PUFA. Increases in placental resolvin and protectin levels may enable the placenta to better handle/resolve inflammation in response to an inflammatory challenge like infection.

The biosynthesis of PUFA derived lipid mediators increases and changes with time. PGs and LTs are produced first to initiate inflammation but as time goes on there is increased recruitment of noninflammatory monocytes and MФs and lipid class switching to SPMs that promote resolution (Serhan and Petasis, 2011). Dietary $\omega-3$ PUFAs can increase the amount of available $\omega$-3 PUFAs and accelerate the switch from pro-inflammatory PG and LT production to inflammation resolving SPMs.

Resolution of inflammation is now considered a coordinated program of events initiated a few hours after the response begins. Acute inflammation is characterized by infiltration of granulocytes, with neutrophils being the first responders, followed by monocytes that mature into M1 MФs in the inflammatory environment that then effect the functions of resident MФs (Serhan and Savill, 2005). Resolution of inflammation involves stopping neutrophil infiltration into the inflammatory site, increasing monocyte/MФ recruitment to the site to phagocytosis the pathogen and apoptotic neutrophils without increasing inflammatory mediator release, and the return of the tissue mononuclear cell population (lymphocytes and macrophages) to pre-infiltration numbers and phenotypes (M2 MФ) (Walker et al., 2011).

Even as the inflammatory response is being initiated, signaling pathways to terminate the response are being programmed. When granulocytes enter tissues they promote the switch of AA-derived PGs and LTs to LXs, which initiate the termination sequence. The lipid mediators being produced depend on the types of cells being encountered in the environment; neutrophils can interact with other leukocytes, platelets, endothelia, mucosal epithelia, interstitial cells, and fibroblasts (Serhan and Savill, 2005). PGE 2 and $\mathrm{PGD}_{2}$ produced at the inflammatory site switch on transcription of enzymes needed to make eicosanoids from AA other than PGs and LTs (Levy et al., 2001). Neutrophils respond to PGs by transcribing the gene for 15-LOX which leads to the temporal production of LXs from AA (Levy et al., 2001). 
Lipoxins halt neutrophil infiltration into inflammatory sites (Serhan et al., 1995) by reducing vascular permeability (Takano et al., 1998), promoting nonphlogistic infiltration of monocytes (Maddox and Serhan, 1996), stimulating MФ to ingest apoptotic neutrophils (Godson et al., 2000) and reducing DC motility and IL-12 production (Aliberti et al., 2002). LXs initiate the termination sequence but they are not the only players, resolvins and protectins are formed from $\omega-3$ PUFAs that are also involved in stopping neutrophil infiltration and neutrophil apoptosis (Serhan and Savill, 2005). As MФs clean up the area by phagocytosing apoptotic cells they are stimulated to release anti-inflammatory (IL-10) and reparative mediators and stop producing PIM (TNFa, IL-1 $\beta$, IL-8, GM-CSF, TxB2, LTC4) (Voll et al., 1997; Fadok et al., 1998; Byrne and Reen, 2002). The process of phagocytosing apoptotic cells stimulates the production of TGF $\beta, \mathrm{PGE}_{2}$, and PAF which may mediate the decrease in PIC production as the addition of these factors to LPS stimulated cells decreased cytokine production (Fadok et al., 1998). TGF $\beta$ has been shown, in vitro and in vivo studies, to be released by these MФs which can then suppress proinflammatory signaling from TLRs (Byrne and Reen, 2002). Phagocytosing apoptotic cells inhibits MФs from killing resident tissue cells (Duffield et al., 2001) and triggers VEGF secretion that is involved in repair of endothelial and epithelial damage that occurred during the inflammatory response (Golpon et al., 2004).

The mechanisms of: PGs signaling lipid class switching in neutrophils, the production of SPMs,

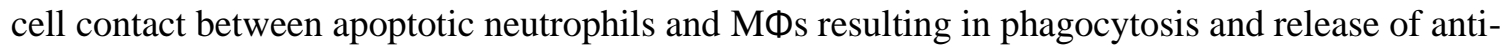
inflammatory cytokines and reparative mediators links mechanisms of inflammation resolution with tissue repair and cell clearance. Phagocytosis of apoptotic cells also stimulates MФs to emigrate and the departure of M1 MФs from the response site to local lymph nodes signals the end of the antiinflammatory program (Bellingan et al., 1996).

\section{Peroxisome Proliferator-Activator Receptors}

Peroxisome proliferator-activator receptors (PPARs) are nuclear receptors and ligand activated transcription factors that form heterodimers with retinoic acid receptors (Dinarello, 2010; Lian et al., 2015). PPARs have roles in development and differentiation as well as modulation of fat and glucose metabolism and the inflammatory response (Clark et al., 2000; Feige et al., 2006; Dinarello, 2010; Li et al., 2014; Lian et al., 2015). Ligands for the receptors include naturally occurring FAs and eicosanoids (Forman et al., 1995). Specfically, PPARy is activated by PUFAs as well as the bioactive metabolite of prostaglandin $\mathrm{D}_{2}, 15$ deoxy $\Delta^{12,14}$ Prostaglandin $\mathrm{J}_{2}\left(15 \mathrm{dPGJ}_{2}\right)$ and 15-HETE (Forman et al., 1995; Bensinger and Tontonoz, 2008). PPAR agonists reduce expression of several PICs, chemokines, and adhesion molecules and effect $\mathrm{T}$ cell proliferation (including in the placenta) by interfering and inhibiting other transcription factors (AP-1, NF-kB, NFAT (nuclear factor of activated T Cells) (IL-2 transcription), 
STATs (STAT3-IL-6 transcription)) by physically interacting with them in a process termed transrepression (Ricote et al., 1998; Clark et al., 2000; Lappas et al., 2002; Genolet et al., 2004; Feige et al., 2006; Szanto and Nagy, 2008; Dinarello, 2010; Li et al., 2014; Lian et al., 2015). Therefore, another way for $\omega$-3 PUFAs to have anti-inflammatory effects is through upregulation and signaling through PPAR $\gamma$.

In addition to its role in lipid metabolism, PPAR $\gamma$ controls inflammation through inhibition of inflammatory gene expression including IL-1 $\beta$, TNF $\alpha$, IL-6, CXCL8, COX-2, VCAM-1, NOS, MMP, and APPs (Ricote et al., 1998; Jiang et al., 1998; Jackson et al., 1999; Takano et al., 2000; Wang et al., 2001). PPAR $y$ also prevents the release of PICs, matrix metalloproteinase activity and vascular smooth cell migration (Bensinger and Tontonoz, 2008; Szanto and Nagy, 2008). By inhibiting transcription of monocyte chemoattractant protein-1 (MCP-1) and its receptor CCR2 in MФs, Peroxisome proliferatoractivator receptor $\gamma$ can inhibit MФ recruitment to inflammatory sites (Szanto and Nagy, 2008). PPAR $\gamma$ can regulate the response of MФs, and T cells to inflammatory stimuli as well as influence the polarization of Th1 versus Th2 cells (Genolet et al., 2004). Peroxisome proliferator-activator receptor $\gamma$ decreases IL-2, INFy, and TNF $\alpha$ production by Th1 cells and increases IL-4, IL-10, and GATA3 production by $\mathrm{T} 2$ cells (Genoler et al., 2004).

There are several endogenous agonists including prostacyclin in addition to many synthetic ligands for PPARץ (Dinarello, 2010). Dasu et al (2009) investigated the anti-inflammatory effects of the synthetic PPARY agonist pioglitazone on human blood monocytes in vitro when exposed to TLR 2 and TLR 4 ligands after pretreatment with pioglitazone. Compared to control, the PPAR $\gamma$ agonist reduced gene expression of TLR2 and TLR4 in addition to reducing TLR ligand-induced binding activity of NF$\mathrm{kB}$, and the amount of PICs and MCP-1 secreted from the cells. In mice treated with pioglitazone for 10 days, peritoneal MФs had reduced expression of TLR2 and TLR4, a decrease in MyD88 dependent signaling, a decrease in NF-KB DNA binding activity, and reduced serum levels of PICs and MCP-1 (Dasu et al., 2009). These in vitro and in vivo studies show that activation of PPAR $\gamma$ has antiinflammatory effects by reducing TLR signaling resulting in reduced PIC production. N-3 PUFAs are endogenous nuclear receptor ligands that activate PPARs and feeding mice $\omega-3$ PUFAs has been show to increase expression of PPARY associated with a reduction in inflammation and disease severity (Li et al., 2014; Lian et al., 2015). Li et al (2014) used the cecal ligation and puncture sepsis model in mice fed fish oil or a control diet. After the procedure, fish oil fed mice had lower plasma TNFa, and IL-6, and lower $\mathrm{PGE}_{2}$ and TNFa in peritoneal lavage fluid. In addition, it was reported that in fish oil fed mice the DNAbinding activity of PPAR $\gamma$ was increased as well as the mRNA expression of IкB $\alpha$ while NF-кB p65 DNA binding activity and iNOS protein levels were reduced in the liver (Li et al., 2014). 
With regard to MФs, PPAR $ү$ promotes alternative activation of the cells (M2 MФ) by increasing expression of target genes involved in $\mathrm{M} 2 \mathrm{M} \Phi$ function and preventing M $\Phi$ s from being activated in response to PIMs (Genoler et al., 2004; Bensinger and Tontonoz, 2008). Classical MФs are activated by PICs and infectious agents and produce PIMs. M2 MФ develop from monocytes in response to IL-4 and have a suppressive phenotype characterized by diminished production of PICs and the ability to inhibit T cell proliferation and promote inflammatory resolution (Szanto and Nagy, 2008). PPAR $ү$ increases MФ production of CXCL8 and IL-1Ra and decreases MФ production of IL-1 $\beta$, IL-6, IL-12, IL-15, TNF $\alpha$, iNOS, IP-10, RANTES, and MIP-1 $\alpha$ (Genoler et al., 2004). PPAR $\gamma$ activation in MФ inhibits their response to LPS or IFN $\gamma$ (Ricote et al., 1998). These actions of PPAR $\gamma$ may be due to PPAR stimulating the breakdown of inflammatory eicosanoids through induction of $\beta$-oxidation or through the ability to transrepress other transcription factors such as NF-кB, AP-1, and STAT (Takano et al., 2000; Wang et al., 2001; Daynes and Jones, 2002). DHA, a fatty acid ligand of PPAR $\gamma$, induces mRNA expression and nuclear translocation of the transcription factor in MФs (Chang et al., 2015). In a PPAR $\gamma$ dependent manner, DHA polarizes MФ to a M2 phenotype but the transcription factor does not seem to account for DHA's ability to inhibit LPS-induced classical macrophage activation (M1) (Chang et al., 2015).

With regard to DCs, activation of PPAR $\gamma$ leads to reduced ability to be stimulated by TLR agonists, reduced capacity to stimulate T cell proliferation, and inhibition of production of IL-12, IL-15, IL-6, TNF $\alpha$, CXCL10 (IP-10), and CCL5 (RANTES); PICs/cytokines for Th1 polarization and chemokines for Th1 cell recruitment (Faveeuw et al., 2000; Gosset et al., 2001; Appel et al., 2005). With regards to co-stimulatory molecule expression, PPAR $\gamma$ activation reduces CD80 expression but induces CD86 expression, a situation that favors Th2 polarization over Th1 polarization of naïve T cells (Genolet et al., 2004). CCR7, the chemokine receptor involved in DC migration, is also reduced with PPAR $\gamma$ activation, limiting DC migration to lymph nodes. Altogether, PPAR $\gamma$ activation in DCs will result in less antigen presentation to naïve T cells, a shift towards Th2 versus Th1 polarization, a reduction in Th1 recruitment, and reduced inflammation. Stimulation of human monocyte-derived DCs with PPAR $\gamma$ agonists decreased secretion of the Th1 promoting cytokine IL-12 when cells were also stimulated with LPS or CD40L compared to DCs only stimulated with LPS or CD40L (Gosset et al., 2001). The PPAR $\gamma$ agonists also down regulated synthesis of IL-10 and RANTES, chemokines that attract Th1 lymphocytes (Gosset et al., 2001), suggesting PPARץ may regulate DCs function so that DCs do not cause naïve CD4+ $\mathrm{T}$ cells to differentiate into Th1 cells and do not recruit Th1 cells to the infection/inflammatory site.

Peroxisome proliferator-activated receptors are expressed by trophoblast cells, in which they have roles in placental development and function (Barak et al., 2008). PPAR $\gamma$ specifically has been reported to 
have a role in trophoblast differentiation and maturation, in regulating the thickness of the spongiotrophoblast layer, affecting the labyrinthine vasculature, enhancing fatty acid uptake and expression of fatty acid transport proteins (Schaiff et al., 2007; Barak et al., 2008; Wieser et al., 2008). A diet high in $\omega$-3 PUFAs, ligands for PPAR $\gamma$, may increase the anti-inflammatory potential of the uterine environment.

\section{Dietary Supplementation with Omega-3 Polyunsaturated Fatty Acids}

A diet high in $\omega-3$ PUFA can be anti-inflammatory for several reasons. First, the $\omega-3$ PUFAs will partially replace the $\omega-6$ PUFA AA in cell membranes which will lead to reduced production of AAderived mediators. Eicosanoids are produced from FAs liberated from membrane phospholipids. AA is converted into 2-series PGs, thromboxanes, and 4-series LTs; by reducing the amount of AA in membranes there is less substrate to be turned into these mediators (Calder, 2003). The PUFAs will also compete for the COX and LOX enzymes and EPA has been reported to competitively inhibit oxygenation of AA by COX (Obata et al., 1999). $\Omega$-3 PUFAs can also suppress cytokine-induced expression of COX-2 and 5-LOX gene expression (Curtis et al., 2000; Curtis et al., 2002), limiting the production of eicosanoids. Altering the FA composition of cell membrane phospholipids can influence membrane activities and cellular responses (Calder, 2003). The FA composition of phospholipids will effect membrane fluidity (Stubbs and Smith, 1984) which affects the activity of membrane-bound proteins such as receptors, transporters, and enzymes (Murphy, 1990), which will influence how an inflammatory cell responds to a stimulus (Grimble, 1998). Membrane phospholipids are used to produce the intracellular signals DAG, inositol phosphate, and ceramide; the FA composition of the substrate phospholipids can influence the production of these signals by phospholipase enzymes and signal transduction pathways (Miles and Calder, 1998).

Omega-3PUFAs will suppress production of PICs and modulate adhesion molecule expression by controlling gene expression of these factors (Calder, 2003). Suppling EPA and DHA in culture inhibited IL-1 $\beta$ and TNF $\alpha$ production by monocytes (Calder, 1997) and the production of IL-6 and CXCL8 by venous endothelial cells (de Caterina et al., 1994; Khalfoun et al., 1997). Feeding fish oil to mice decreased circulating levels of TNF $\alpha$, IL-1 $1 \beta$, and IL-6 after an LPS challenge compared to mice fed safflower oil (Sadeghi et al., 1999). With regards to adhesion molecules, human venous endothelial cells cultured with EPA or DHA had decreased surface expression of E-selectin, ICAM-1, and VCAM-1 when stimulated with cytokines or LPS (de Caterina et al., 1994) and had diminished adhesion of ligandbearing monocytes (de Caterina and Libby, 1996). Monocytes cultured with EPA also displayed less surface ICAM-1 when stimulate with IFNץ (Hughes et al., 1996). The decrease expression of VCAM-1 
on the surface of endothelial cells was shown to be due to DHAs effect of gene expression of the adhesion molecule (de Caterina et al., 1994).

In cultured bovine chondrocytes, EPA, DHA, and ALA decreased cytokine induced expression of COX-2 (but not COX-1), IL-1 $\alpha$, and TNF $\alpha$ (Curtis et al., 2000). Adding EPA, DHA, or ALA to cultured explants of human osteoarthritic cartilage decreased cytokine induced upregulation of COX-2, 5-LOX, 5LOX activating protein (FLAP), IL-1 $\alpha$, IL-1 $\beta$, TNF $\alpha$, MMP-3, and MMP-13 expression (Curtis et al., 2002). Gene expression of COX-1, 12-LOX, and 15-LOX were not affected by the $\omega-3$ PUFAs but these genes were not induced by the cytokines anyway (Curtis et al., 2002).

The transcription factor NF-kB is involved in inducing expression of several inflammatory genes including COX-2, ICAM-1, VCAM-1, E-selectin, TNF $\alpha$, IL-1 $\beta$, NOS, MMPs, and APPs (Christman et al., 1998; Chen et al., 1999; Calder, 2002). This transcription factor is located in the cytosol of resting inflammatory cells as an inactive heterodimer. When stimulated, a signaling cascade activates the IкB

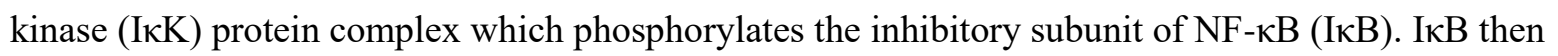
dissociates from the rest of the inactive NF- $\kappa$ B trimer (Karin and Ben-Neriah, 2000; Karin and Helhase, 2000) and is degraded while the remaining NF- $\mathrm{KB}$ heterodimer translocates into the nucleus to bind to response elements of target genes regulating transcription. $\Omega-3$ PUFA may regulate gene expression by limiting NF-кB activation. When the MФ cell line RAW264.7 was cultured with EPA and then stimulated with LPS, the cells produced less TNF $\alpha$ mRNA and protein, and less $\mathrm{PGE}_{2}$ which was associated with reduced NF-kB activity (Lo et al., 1999).

Feeding fatty acids to cows after the post-partum period of immune-suppression and uterine involution may have benefits to future pregnancy. Specific fatty acids could suppress the biosynthesis of inflammatory molecules reducing residual inflammatory responses in the uterus to subclinical endometritis and preventing PGF $_{2 \alpha}$ production and luteolysis (Thatcher et al., 2010).

The unsaturated fatty acid oleic acid (18:1 n-9) can be made by animal tissues, however, linoleic acid (18:2 n-6) and ALA (18:3n-3) are considered dietary essential as the required desaturases to produce these FAs are not present in animals (Abayasekara and Wathes, 1999) Linoleic acid (20:4 n-6) undergoes elongation and desaturation to form differential n-6 products, such as dihomo- - linolenic (20:3 n-6) and AA (20:4n-6). ALA (18:3n-3) undergoes elongation and desaturation to form n-3 products such as EPA (20:5n-3) and DHA (22:6n-3). AA can be used to produce eicosanoid products, different series of PGs $\left(\mathrm{PGF}_{1}, \mathrm{PGF}_{2}, \mathrm{PGF}_{3}\right.$ ), thromboxanes, and LTs (Dennis and Norris, 2015) ALA is found as a component of chloroplast lipids and is high in linseed oil from the flax plant (Linum usitatissimum) (Sergent, 1997). 
The diet of ruminants contains predominantly unsaturated fatty acids but upon ingestion, dietary lipids are extensively hydrolyzed in the rumen and the unsaturated fatty acids are biohydrogenated by rumen microbes to more saturated products (Wonsil et al., 1994). Supplementation of nonprotected fats can decrease dry matter intake (DMI) and reduce digestibility of non-lipid energy sources in ruminants due to changes in ruminal fermentation, gut motility, palatability, release of gut hormones, and oxidation of fat in the liver (Allen, 2000). Both amount and form of a fat supplement matter when it comes to rumen health, DMI, and digestibility. Flaxseed (Linum usitatissimum) on a dry matter (DM) basis contains $20 \%$ crude protein (CP), 18\% neutral detergent fiber (NDF), and 40\% ether extract. It is rich in ALA (18:3n-3), averaging $18 \%$ of the total seed weight and constituting 53\% of the total fatty acids (Mustafa et al., 2002). Being an oilseed, the high level of PUFAs in flaxseed can negatively affect ruminal fiber digestion (Palmquist and Jenkins, 1980).

Gonthier et al (2004) using a Latin square design fed lactating Holstein cows a control diet with no flaxseed at a 64:36 forage: concentrate ratio (DM basis) or flaxseed (12.6\% dietary DM) added as a topdressing at a 55:45 forage: concentrate ratio (DM basis) in 3 different forms (raw, micronized, and extruded). The study reported that flaxseed supplementation improved post-ruminal organic matter and total-tract digestibility (expressed as a percent of passage to duodenum and percent of intake, respectively) compared to a diet with no flaxseed, with no harmful effects on rumen function. Schroeder et al (2014) using a Latin Square design fed Holstein steers basal diets of $47 \%$ concentrate, $30 \%$ corn silage, $17 \%$ alfalfa hay and $6 \%$ beet pulp shreds. The concentrate portion contained either $7.5 \%$ linseed meal (control), 10\% whole flaxseed, $10 \%$ rolled flaxseed, or $10 \%$ ground flaxseed. Dry matter intake was similar between diets and treatments had no effect on apparent or true OM digestion in the rumen. Large intestine, small intestine, and total-tract digestibilities were similar for the treatments. There was no change in protein digestion with the addition of flaxseed supported by no differences in duodenal CP flow (bacterial., apparent feed, or total), ileal CP flow, fecal CP output, microbial efficiency, or CP digestibility (apparent ruminal., true ruminal., small intestine, large intestine, or total tract) (Schroeder et al., 2014). Gonthier et al (2004) reported a reduction in microbial CP flow to the duodenum and microbial efficiencies (true and apparent) with flaxseed supplementation suggesting flaxseed can reduce ruminal microbial growth. However, ruminal digestion of $\mathrm{CP}$ was not affected by diet and post-ruminal $\mathrm{CP}$ digestibility (percentage of passage to the duodenum) and total tract $\mathrm{CP}$ digestibility were greater with the flaxseed diets (Gonthier et al., 2004). Flaxseed had no effect on total-tract neutral detergent fiber (NDF) or acid detergent fiber (ADF) digestion at the level of 10\% of dietary DM and no differences in NDF and ADF intake, duodenal flow, ileal flow, fecal output, or digestibility were detected in the Schroeder et al study (2014). Gonthier et al (2004) reported the addition of flaxseed (12.6\% of dietary DM) decreased ruminal ADF digestibility, increased post-ruminal ADF digestibility and had no effect on total tract ADF 
digestibility (expressed as a percentage of intake). Flaxseed tended to increase post-ruminal and total tract digestibilities of DM, OM, NDF, and gross energy (Gonthier et al., 2004). Gonthier et al (2004) reported reduced microbial efficiency with raw, micronized, and extruded (12.6\% of diet DM) flaxseed compared to the control diet without flaxseed but Schroeder et al (2014) feeding flaxseed at 10\% DM reported no change in microbial efficiency (g of microbial N/kg of OM truly fermented). Schroeder et al (2014) reported no effect of feeding flaxseed on ruminal $\mathrm{pH}$, ammonia, or volatile fatty acid (VFA) concentrations (total, individual, acetate: propionate, or acetate: butyrate: propionate) indicating similar fiber digestion and ruminal fermentation with the different diets. Consistent with this, Gonthier et al (2004) reported no difference in average ruminal $\mathrm{pH}, \mathrm{NH}_{3} \mathrm{~N}$, or total VFA. In the Gonthier et al (2004) study there was a decrease in the molar proportion of acetate and an increase in the molar proportion of propionate when feeding flaxseed which decreased the acetate: propionate ratio. The authors stated that it was not clear whether the differences in molar proportions of the two VFAs was due to flaxseed supplementation or differences in forage level, as the 3 flaxseed diets had a different forage: concentrate ratio than the control diet. Lower acetate and greater propionate proportions with the addition of oilseeds to ruminant diets tends to relate to lower ruminal fiber digestion fitting with the reported reduction in ruminal ADF digestibility (expressed as a percentage of ADF intake) with the flaxseed diets compared to control (Gonthier et al., 2004).

Petit et al. (2002) fed primiparous lactating Holstein cows one of 3 total mixed diets with a fat supplement based on either whole flaxseed (FLA; 10.4\% DM), Megalac (MEG; 3.8\% DM), or micronized soybeans (SOY; 17.7\% DM). Cows fed the whole flaxseed had higher milk production than those fed MEG, higher milk protein percentage than those fed MEG or SOY, and higher levels of ALA in their milk compared to MEG cows. Cows fed flaxseed had a lower DM digestibility compared to the SOY group, and lower ether extract, ADF and NDF digestibility compared to the SOY and MEG groups. Analyzing blood collected from the cows, FLA cows had reduced levels of NEFAs, total cholesterol $(\mathrm{mg} / 100 \mathrm{ml})$ and HDL cholesterol $(\mathrm{mg} / 100 \mathrm{~mL})$ compared to the other two diets. With regards to the fatty acid profile in the blood samples, compared to the SOY and MEG groups, the FLA cows had lower levels of palmitate (16:0) but higher levels of stearic (18:0), ALA (18:3n-3), and EPA (20:5 n-3) with a reduced omega-6/omega-3 ratio (Petit et al., 2002).

Petit and Côrtes (2010) fed multiparous Holstein dairy cows one of four iso-net energy total mixed rations that contained either $21 \mathrm{~g} / \mathrm{kg}$ DM calcium salts of palm oil (CON), $72 \mathrm{~g} / \mathrm{kg}$ DM whole flaxseed (WHO), $72 \mathrm{~g} / \mathrm{kg}$ DM ground flaxseed (GRO), or $36 \mathrm{~g} / \mathrm{kg}$ DM whole flaxseed and $36 \mathrm{~g} / \mathrm{kg} \mathrm{DM}$ ground flaxseed (MIX). The GRO group had reduced DM intake compared to the other 3 diets and might have been due to disturbances in rumen function, this diet contained the largest amount of flaxseed with a 
disturbed seed coat leaving the oil unprotected from rumen microbes. The CON group had reduced milk lactose compared to the 3 flaxseed diets while milk fat and CP showed no differences between diets. Among long chain fatty acids, ALA results in high rates of gluconeogenesis which ruminants use for lactose production. Increased gluconeogenesis with flaxseed supplementation may explain the increase in milk lactose in the 3 diets containing flaxseed. With regards to the fatty acid milk profile, all 3 flaxseed diets decreased the amount of palmitate (16:0), increased the amount of stearic acid (18:0), oleic acid (18:1 cis 9), ALA (18:3 cis 9,12,15), increased the level of mono unsaturated fatty acids, long chain fatty acids, and omega-3 fatty acids, while decreasing the level of saturated fatty acids and the omega6/omega-3 ratio. The GRO and MIX diets also significantly increased the levels of poly-unsaturated fatty acids while the WHO and MIX diets significantly decreased the omega 6 FA levels compared to CON (Petit and Côrtes, 2010).

Scholljegerdes and Kronberg (2008) using a Latin Square design fed Angus Heifers hay without a supplement or supplemented with $0.91 \mathrm{~kg} / \mathrm{d}$ (3.2\% of added dietary fatty acids, DM basis) of whole flaxseed, or $1.82 \mathrm{~kg} / \mathrm{d}$ (5.8\% of added dietary fatty acids, DM basis ) of whole flaxseed on a DM basis. In agreement with Gonthier et al (2004), Scholljegerdes and Kronberg (2008) reported an increase in apparent lower tract OM digestibility (\% of duodenal flow) with the addition of flaxseed to the diet, however, the apparent total-tract OM digestibility (\% of intake) did not differ with the diets.

Scholljegerdes and Kronberg (2008) reported that flaxseed supplementation increased the apparent lower tract and total-tract $\mathrm{N}$ digestibility indicating whole flaxseed does not impair ruminal $\mathrm{N}$ metabolism and that $\mathrm{N}$ escaping ruminal degradation is available in the small intestine. There were no differences in duodenal or fecal NDF flow and flaxseed had no effect on ruminal, lower tract, or total-tract NDF digestibility (Scholljegerdes and Kronberg, 2008). Going from the control diet to $0.91 \mathrm{~kg} / \mathrm{d}$ to $1.82 \mathrm{~kg} / \mathrm{d}$ of flaxseed, molar proportions of acetate decreased while molar proportions of propionate, isobutyrate, isovalerate, valerate increased, and the acetate: propionate ratio decreased. Ruminal $\mathrm{pH}, \mathrm{NH}_{3}$, total VFAs, and butyrate levels were unaffected by diet. Flax feeding increased intestinal supply of ALA, mono unsaturated fatty acids and PUFAs which may increase tissue supply of unsaturated fatty acids (Scholljegerdes and Kronberg, 2008).

Côrtes et al. (2010) using a Latin square design fed lactating Holstein cows isonitrogenous and isoenergetic diets: a control diet without flaxseed (CON), a diet (on a DM basis) with $4.2 \%$ whole flaxseed (FLA), a diet with $1.9 \%$ calcium salts of flaxseed oil (SAL), or a diet with $2.3 \%$ whole flaxseed and $0.8 \%$ calcium salts of flaxseed oil (MIX) fed ad libitum. Diets did not affect DMI, digestibility, milk production, milk concentrations of protein, lactose, urea N, or total solids. Feeding whole flaxseed did not affect rumen $\mathrm{pH}$, concentration of ammonia $\mathrm{N}$, total VFA, the ratio of acetate: propionate, or molar 
proportions of specific VFAs (Côrtes et al., 2010). Feeding FLA increased the percent of 18:0, and longer chain fatty acids in ruminal fluid. With regard to milk, the percent of 16:0 was decreased with FLA compared to CON, the percent of long chain fatty acids were increased compared to CON and the FLA milk had higher levels of ALA but was not high enough to be significant. The milk ratio of n-6:n-3 was also decreased with the FLA diet compared to CON (Côrtes et al., 2010).

Oba et al. (2009) used a crossover design and fed primiparous Holstein cows unprocessed whole flaxseed (WF) or rolled flaxseed (RF) at $100 \mathrm{~g} \mathrm{Kg}^{-1}$ of dietary DM. DMI, milk yield, and milk composition were similar between diets. Apparent total tract digestibility of ether extract was lower for WF than RF and excretion of ALA in feces was greater with WF than RF. However, concentration of ALA in milk was not different between diets and both diets increased ALA in milk (3x) compared to before the study when cows were fed sunflower seed instead of flaxseed. Results suggest that both WF and RF increase the absorption of ALA to a similar extent despite the lower digestibility of WF. This may be due to reduced lipolysis in the rumen or fatty acid biohydrogenation of WF since the fatty acids are protected by an intact seed coat. This idea is supported by a decreased concentration of vaccenic acid (a fatty acid intermediate formed during biohydrogenation) in milk fat with the WF diet. While dry-rolling flaxseed breaks the seed coat and allows for better access (increased digestibility) to the fatty acids, it also allows for increased biohydrogenation in the rumen, therefore, does not necessarily improve absorption of ALA (Oba et al., 2009). When feeding the unprocessed WF, intact WF appeared in the feces, however, comparing the ether extract in excreted flaxseed to flaxseed before it was ingested there was a 76\% reduction in ether extract suggesting that the fats present in flaxseed were able to be utilized by cattle even though the seed coat appeared intact (Oba et al., 2009). Mesgaran et al (2012) fed lactating dairy cows either ground flaxseed, whole flaxseed or extruded soybeans, in a Latin square design, up to $9 \%$ of diet DM with no differences in regards to DMI, body weight, body condition score, or milk yield. There was no difference between diets in plasma NEFAs or BHB. The ground flaxseed diet increased the levels of ALA, monounsaturated FAs and PUFAs in the milk. Increases in unsaturated FAs in milk when fed a flaxseed diet suggests sufficient quantities of PUFAs in the flaxseed survived bio-hydrogenation in the rumen to be transferred to milk fat (Oba et al., 2009; Mesgaran et al., 2012) and is likely to be available for use by other tissues and incorporation into the plasma membranes of various cells.

Wachira et al. (2000) using a Latin square design fed wether lambs 4 iso-energic and isonitrogenous diets based on dried grass and provided similar fat levels $(60 \mathrm{~g} / \mathrm{kg} \mathrm{DM})$ from 4 different sources. One diet included Megalac (a calcium soap of palm oil) (control; palmitic acid 16:0), one diet provided whole flaxseed (ALA, 18:3 n-3), one provided fish oil (EPA 20:5 n-3; DHA 22:6 n-3) and the last diet provided whole flaxseed plus fish oil (50:50 mix on an oil weight basis). Wethers were fed 
$1.2 \mathrm{~kg} /$ day. With the flaxseed diet, duodenal flow of ALA was double compared to the control diet and also had higher duodenal flow of 18:0 and trans 18:1 FAs. The increase in duodenal flow of the 18:0 and trans 18:1 FAs is likely to be a result of biohydrogenation by rumen microbes and in fact the flaxseed diet had a $92.4 \%$ biohydrogenation rate of ALA compared to $80.2 \%$ with the control diet. The fact that the whole flaxseed had $24.4 \mathrm{~g} / \mathrm{kg}$ DM of ALA compared to $4.5 \mathrm{~g} / \mathrm{kg} \mathrm{DM}$ in the control diet allowed for increased apparent small intestine fatty acid digestibility (duodenum to rectum) of ALA with the whole flaxseed diet. This study incorporated the whole flaxseed into a pellet which damaged the seed coat and provided the rumen microbes access to the fatty acids (Wachira et al., 2000). Had the flaxseed been fed intact, there would have been less biohydrogenation and potentially even greater ALA reaching the small intestine. Feeding whole flaxseed compared to control did not affect whole tract (mouth to rectum) digestibility of DM, OM, CP, NDF, total FAs, or gross energy. The flaxseed diet compared to control did not affect ruminal fluid $\mathrm{pH}$, molar proportions of acetate, propionate, or butyrate, or the ratio of ketogenic to glucogenic acids (acetate + butyrate/propionate) (Wachira et al., 2000). The control diet had a lower duodenal flow of ammonia-nitrogen but a higher flow of non-ammonia nitrogen while microbial nitrogen flow was similar between the control and flaxseed diets. The flaxseed diet compared to control had a higher proportion of OM truly digested in the rumen (Wachira et al., 2000).

Soder et al. (2012) using a Latin Square design supplemented ground flaxseed in a orchardgrass diet (70 g of total DM) at the level of $0 \%$ (70g of herbage), $5 \%$ (5g flaxseed $+65 \mathrm{~g}$ herbage), $10 \%(10 \mathrm{~g}$ flaxseed $+60 \mathrm{~g}$ herbage $)$, and $15 \%(15 \mathrm{~g}$ flaxseed $+55 \mathrm{~g}$ herbage $)$. With increasing flaxseed supplementation, apparent DM, OM, and NDF digestibilities decreased, whereas true DM and OM digestibilities were not affected (Soder et al., 2012). Flaxseed included in the diets was ground breaking the seed coat and allowing the oil to be released in the rumen which may have impacted the rumen microbes and nutrient (OM, DM, and NDF) digestibility. While ruminal $\mathrm{pH}$ and total VFAs were not affected by the flaxseed, molar proportions of acetate and propionate increased while butyrate and valerate decreased with increasing flaxseed supplementation resulting in a decreasing acetate: propionate ratio with increasing flaxseed supplementation. Ammonia-N concentration, apparent CP digestibility, and microbial N synthesis were not affected by diets (Soder et al., 2012).

An in vitro study (Maia et al., 2007) reported that growth of ruminal bacteria are affected by PUFAs which could influence ruminal fermentation, although different species have different levels of sensitives and different PUFA are more damaging than others. Ranking the PUFA tested on toxicity to bacterial growth, the most toxic was EPA, followed by DHA, ALA, and finally linoleic acid (Maia et al., 2007). ALA is toxic for the 3 predominant cellulolytic species responsible for fiber digestion (Fibrobacter succinogenes, Ruminococcus albus, and Ruminococcus flavefaciens) by disrupting cell 
integrity (Maia et al., 2007). PUFA may be directly toxic to methanogens that use hydrogen for $\mathrm{CH}_{4}$ production. With less methanogens there may be more free hydrogen which can inhibit growth of cellulolytic bacteria and impair fiber digestion (Soder et al., 2012). However, microbial N-flow was not changed by increasing supplementation of flaxseed in Soder et al's (2012) study suggesting that if there is a reduction in methaogenic and cellulolytic bacteria that other microbial species are compensating. When feeding whole seeds the fatty acids are protected by the seed coat minimizing PUFA toxicity to rumen bacteria and biohydrogenation by the bacteria, potentially increasing their availability in the small intestine.

\section{Omega-3 PUFAs and Benefits to Reproduction}

Fat supplementation in rumen diets is used to increase the energy density of the diet and to utilize the properties of specific fatty acids. Several studies using different strategies of fat supplementation have reported benefits in health, reproduction, and production of ruminants (Staples et al., 1998; Santos et al., 2008; Silvestre et al., 2011a; Greco et al., 2015; Rodney et al., 2015). The benefits are not just due to increased energy provided by the FAs but by the properties of the specific FAs in the diet (Staples et al., 1998). Altering the lipid content of the diet can shift the FA profile of tissues and alter tissue physiology (Calder, 2012). Indeed, feeding high amounts of omega-3 FAs to cows resulted in a higher proportions of omega-3 FAs in the endometrium concurrent with a lower proportion of AA (Burns et al., 2003; Bilby et al., 2006c). Reduced amounts of available AA limit the PG response to a stimulus (Mattos et al., 2002). Thus, most research investigating the effect of dietary FAs on uterine biology focus on changing the endometrial FA profile to reduce the proportion of PG precursors (ex: arachidonic acid) and thus control the amount the 2 series PGs that can be produced. Because PGF $_{2 \alpha}$ is luteolytic in ruminants, limiting the amount of $\mathrm{PGF}_{2 \alpha}$ should protect the CL and $\mathrm{P}_{4}$ production from the CL (Ribeiro et al., 2016c). In general., studies using dietary FA supplementation report benefits with regards to reproduction (Rodney et al., 2015 ) with the benefits occurring not only from additional energy but from non-caloric effects of FAs (Santos et al., 2008). Non-caloric effects include altering the FA profile at the maternal-fetal interface (limiting series 2 prostaglandin precursors), immune modulating functions, altered follicle development, changes in oocyte quality, and improved uterine environment (Ribeiro et al., 2016c).

Postpartum diseases in cattle are a major factor in reproductive failure with cows suffering inflammatory diseases having impaired hypothalamic-pituitary-ovarian axis function, impaired conceptus development, and increased rates of embryo loss (Ribeiro et al., 2016b). Not only is an inflammatory environment not conducive to conceptus development but prostaglandins are mediators of an inflammatory response. Since the precursors of prostaglandins are FAs, in a pro-inflammatory environment not only will there be an increase in inflammatory prostaglandins but a change in tissue lipid 
content. During elongation, the conceptus needs lipid for energy as well as for incorporation into new cell membranes as cells proliferate. Therefore, an inflammatory environment is not only hostile to an embryo but will limit the amount of lipid available for embryo growth. Dietary supplementation of FAs not only provides energy for the dam and embryo but depending on the type of FAs added to the diet, the amount of inflammatory prostaglandins produced can be reduced and more lipid can be available for conceptus growth.

Feeding fish meal, which is high in the omega-3 FAs EPA and DHA improved pregnancy per AI in lactating dairy cows (Armstrong et al., 1990; Burke et al., 1997). When Silvestre et al (2011) fed lactating dairy cows fish oil (high in n-3) or palm oil (no n-3; little n-6) around the time of breeding they saw a reduction in pregnancy loss to first $\mathrm{AI}$ in their fish oil fed cows and an increase in pregnancy to second AI. These two results together means fish oil improved the accumulated proportion of pregnant cows after 2 postpartum inseminations (Silvestre et al., 2011). Feeding whole flaxseed (10.4\% DM), which can be turned into EPA and DHA, to cows has been seen to increase first service pregnancy per AI (Petit and Twagiramungu, 2006) and to reduce pregnancy loss (Ambrose et al., 2006 (rolled flaxseed 9\% DM); Petit and Twagiramungu, 2006).

The change in pregnancy success in cows fed PUFAs may be due to immune modulating effects of PUFAs which have been seen to have beneficial effects with regards to inflammation (Calder 2006a,b; 2013a,b,c) and increased amounts of fatty acids are found in the uterus/placenta after supplemented feeding. Therefore, feeding PUFAs may result in these fatty acids being able to have direct effects on immune cells in the reproductive tract leading to alteration of the immune response in that area. In the study that supplemented cows with fish oil, neutrophils isolated from blood samples taken around the time of insemination had a highly attenuated production of TNF- $\alpha$ when stimulated with LPS while the neutrophils from palm oil supplemented cows had a huge increase in TNF- $\alpha$ when stimulated with LPS (Silvestre et al., 2011a; Silvestre et al., 2011b). Greco et al (2015) fed lactating dairy cows diets that were formulated with a mixture of oils (fish, safflower, and palm) to produce diets with varying ratios of n-6 to n-3 fatty acid concentrations and then evaluated their inflammatory response to an infusion of LPS into one quarter of the mammary gland. Cows fed the lowest n-6/n-3 ratio (ate the most omega 3 fatty acids) had the lowest level of IL-6 after LPS and had an attenuated increase in somatic cell count while the cows fed the diet with the highest n-6/n-3 ratio had significantly higher haptoglobin levels in response to LPS (Greco et al., 2015).

Vijay-Kumar et al. (2011) fed mice either a diet with fish oil (FO) or saturated fat (SF) before 2 LPS challenges 7 days apart (100ng of LPS followed by $500 \mathrm{ng}$ of LPS). Mice fed the saturated fat diet had higher mortality after the LPS challenges and lost more body weight. With regards to the PIC IL-1 $\beta$, 
although not significant different, the FO mice had lower levels compared to the SF mice before LPS challenge (100ng) and levels barely increased in FO mice in response to LPS challenge. In the SF mice, there was an increase in IL-1 $\beta$ in response to LPS challenge and a significant difference in IL-1 $1 \beta$ levels between FO and SF groups after LPS challenge. With regards to behavior, after the 100ng LPS challenge the SF mice became reclusive and went to sleep while the FO mice displayed no abnormal behaviors. After the 500ng challenge both groups were moribund, however, mice in the FO group recovered more rapidly than the SF mice. (Vijay-Kumar et al., 2011).

Caughey et al. (1996) saw a decrease in TNF $\alpha$, IL-1 $\beta$, thromboxane $\mathrm{B}_{2}$, and $\mathrm{PGE}_{2}$ production by mononuclear cells (in response to LPS stimulation) of male subjects that added flaxseed oil to their diet for 4 weeks but subjects that added sunflower oil to their diet had no change in production of the PIM. There was an association between PIM production and the EPA content of their cells where cytokine production decreased as cellular EPA increased to $~ 1 \%$ of total fatty acids (Caughey et al., 1996). These studies suggest that PUFAs, specifically $\omega$-3 fatty acids, may dampen the inflammatory and APR to bacterial components, which would be favorable during pregnancy when an immunosuppressive environment in the uterus is needed for embryo survival.

A study that fed lactating dairy cows either calcium salts of fish oil (11\% of C20:5n-3 + C22:6n3 ) or calcium salts of palm oil (47\% c16:0) during the breeding period (30-80 days post-partum) found that neutrophils (isolated at 80 days post-partum) cultured with LPS from cows supplemented with fish oil produced significantly less TNF $\alpha$ than neutrophils isolated from cows supplemented with palm oil. In addition, neutrophils isolated from cows fed fish oil contained significantly higher amounts of omega 3 fatty acids (C20:5n-3, C22:5n-3, C22:6n-3) giving them a lower n-6/n-3 fatty acid ratio than neutrophils isolated from cows fed the palm oil (Silvestre et al., 2008). This suggests that neutrophils in the fish oil fed cows were less inflammatory which could be beneficial when trying to establish a pregnancy, especially in a dairy cow that may have subclinical endometritis.

In vitro, ALA was shown to have beneficial effects on embryo development with regards to the percentage of cumulus-oocyte complexes that cleaved and made it to the blastocyst stage and improved the quality of the embryos (Marei et al., 2009). A high proportion of embryonic losses precede or occur during the period when the embryo inhibits uterine secretion of $\mathrm{PGF}_{2 \alpha}$, some of these losses may be due to under-sized embryos the secrete insufficient IFN- $\tau$ to prevent endometrial secretion of $\mathrm{PGF}_{2 \alpha}$ (Bilby et al., 2006a). Pre-implantation embryonic loss can also be attributed to inflammatory mediators such as $\mathrm{TNF} \alpha, \mathrm{IL}-1 \beta$, NO, and $\mathrm{PGF}_{2 \alpha}$, which would be present if the uterus has any bacterial contamination. These PIM hinder embryonic development through damages to the oocyte or developing embryo (Hansen 
et al., 2004). Direct beneficial effects of ALA on the developing embryo (Marei et al., 2009) or through anti-inflammatory effects could prevent early embryonic loss; a sufficiently developed embryo will be able to secrete enough IFN- $\tau$ to prevent luteolysis.

Lian et al. (2015) fed male C57BL/6 mice a low (4.62and DHA and 2.8\% EPA) or high (12.4\% DHA and 7.35\% EPA) $\omega-3$ PUFA diet in the form of fish oil (Menhaden Oil) for 4-12 weeks. After 12 weeks of feeding, the PUFA diets caused proliferation of hepatic natural (but not induced Tregs) Treg cells (CD25+FOXP3+) increasing the percentage of Tregs among CD4+ cells (Lian et al., 2015). The Treg cells also showed higher expression of molecules (CD103 (a TGF $\beta$ inducible surface integrin), CTLA-4, CD62L) involved in their suppressive roles suggesting enhanced immune suppressive functions of Tregs in the fish oil fed mice (Lian et al., 2015). Liver tissue from $\omega$-3 PUFA fed rats had higher IL-10 and TGF $\beta$ expression (Lian et al., 2015), two cytokines that mediate Treg functions and are involved in the generation and maintenance of Tregs (Asseman et al., 1999; Nakamura et al., 2001; Nakamura et al., 2004; Wan and Flavell, 2006). The role of Tregs is to maintain peripheral tolerance, control inflammation, and during pregnancy to prevent rejection of the allogenic fetus. This occurs through cellcell contact between the Treg and another immune cell (they can suppress the other T helper cell subsets) and by Treg production of regulatory cytokines such as TGF $\beta$ and IL-10 (Nakamura et al., 2001; Bettelli et al., 2006; Shevach, 2009). Lian et al (2015) administered Concanavalin A to mice after 6-8 weeks of eating the $\omega-3$ PUFA or control diets to elicit hepatitis and investigate beneficial effects of the diets. Mice fed the fish oil had more hepatic Tregs and less liver damage as assessed by liver histology analysis. Fish oil fed mice also had increased liver PPAR $\gamma$ and TGF $\beta$ (did not reach significance) expression and decreased expression of PICs (IL-1 $\beta$, IL-6, TNF $\alpha$, IFN $\gamma$ ).

Upregulation of PPAR $y$ by dietary $\omega-3$ PUFAs may be mediating the increase in Treg cells as Lian et al (2015) saw an increase in liver PPAR $y$ along with increased liver Tregs when feeding mice fish oil and Cipolletta et al (2012) knocked out PPAR $\gamma$ in mouse Treg cells and concluded that PPAR $\gamma$ was critical for accumulation of visceral adipose tissue Tregs and for Treg phenotype. IL-10 and TGF $\beta$ are not only produced by Tregs to mediate their suppressive functions (Asseman et al., 1999; Hara et al., 2001; Nakamura et al., 2004; Wan and Flavell, 2006) but along with IL-2 are involved in polarization of naïve CD4+ T cells to a Treg phenotype and maintenance of the phenotype (Wan and Flavell, 2006). TGF $\beta$ in conjunction with IL-2 activate the transcription factors STAT5 and FOXP3 which polarize a naïve CD4+ into a Treg (Bettelli et al., 2006; Murphy, 2012). In a mouse model of atopic dermatitis, administration of fermented fish oil (in drinking water) reduced skin inflammation due to increased expression of TGF $\beta$, IL-10, and FOXP3 as well as the number of Treg cells at the inflammation site (Han et al., 2012), 
suggesting the $\omega-3$ PUFA increased Tregs by upregulating FOXP3 or TGF $\beta$ which then activated FOXP3 expression and the increased Tregs reduced inflammation in the fermented fish oil group.

Jaudszus et al. (2013) cultured human PBMCs with EPA or DHA and reported immunosuppressive and pro-resolving effects of the $\omega-3$ PUFAs. Intracellular levels of IL-2, TNF- $\alpha$, and IL-4 were reduced in T helper (Th) cells when incubated with either $\omega$-3 PUFA, although EPA had a greater effect. This anti-inflammatory effect of the $\omega-3$ PUFAs involved PPAR $\gamma$ as a mediator as a PPAR $\gamma$ antagonist reversed the effects. Levels of IFN $\gamma$ were unchanged by the $\omega-3$ PUFAs (Jaudszus et al., 2013). With regards to pro resolving functions, the $\omega$-3 PUFAs increased the population of IL-10 positive monocytes. Incorporation of EPA and DHA into cellular membranes was shown to be at the expense of AA which resulted in a change in the lipid mediators produced. Cells stimulated with LPS after incubation with the $\omega-3$ PUFAs produced decreased amounts of PGE $_{2}$, thromboxane B2, LTB4, and 12-HETE and instead 18-HETE (can be metabolized into the E-series resolvins) and RvD1 were produced.

In summary, the above studies demonstrate that $\omega$-3 PUFAs can influence the response to a stimulus with regard to the type of PGs produced, the production of SPMs, the production of Tregs, and the function of immune cells; all of which factor in to mediating inflammation and maternal tolerance during pregnancy.

\section{Statement of the Problem}

Dairy cows have high rates of early embryonic loss, which influence production, profitability, and culling rates. Parturition and the onset of lactation are associated with a state of negative energy balance that suppresses the immune system. That combined with bacteria entering the reproductive tract during parturition or into the mammary glands makes dairy cows highly susceptible to subclinical and clinical infections. A subclinical bacterial infection can turn into a clinical infection under the influence of progesterone, which is immunosuppressive, and be detrimental to successful pregnancy. While several pro-inflammatory molecules are involved in immunological signaling, stimulating embryonic growth, vascular remodeling of uterine arteries and implantation of the embryo, the timing and quantity of the molecules must be tightly controlled. Immune cells at the maternal-fetal interface have specific phenotypes that regulate maternal tolerance to fetal antigens and mediate vascular remodeling and implantation. An infection will result in the production of inflammatory mediators that can act directly on the developing embryo to impair development or cause apoptosis of embryonic cells. Inflammatory mediators also will affect the phenotype of immune cells resulting in loss of tolerance to fetal antigen and impaired vascular remodeling and implantation. Ultimately, infection during early pregnancy can result in 
embryonic loss. Dietary omega-3 polyunsaturated fatty acids have anti-inflammatory effects and may have reproductive benefits. Using LPS to mimic a gram-negative bacterial infection, the objectives of this study were to identify the immunological changes in response to LPS in early pregnancy in sheep that could be detrimental to embryo survival, to examine whether dietary omega-3 polyunsaturated fatty acids could modulate the immune response to LPS, and to examine whether Dorset and Suffolk ewes have differential immunological responses to LPS. 


\section{Chapter 2: Materials and Methods}

Animals

The first study was conducted in the spring (May 2015) with 16 mature Dorset ewes (10 control, 6 flaxseed) with an average body weight of $66.5 \pm 2.08 \mathrm{~kg}$ and the second study in the fall (AugustSeptember 2015) with 42 mature Dorset ewes (21 control, 21 flaxseed) with an average body weight of $70.96 \pm 1.69 \mathrm{~kg}$. Breed responses to LPS were compared in Fall 2015 between 21 mature Dorset ewes with an average body weight of $71.78 \pm 1.41 \mathrm{~kg}$ and 32 mature Suffolk ewes with an average body weight of $76.73 \pm 2.07 \mathrm{~kg}$. For the fall experiments, ewes were divided into 4 groups in the fall experiments and experimental days were a week apart for 4 weeks with the first and third experimental days using Dorset ewes (half on the flaxseed supplement and half on the control supplement on both days) and the second and forth experimental days using Suffolk ewes. All ewes in the breed study were given the control supplement. Abbreviations used for the treatment groups are CP (control supplement + PBS), CL (control supplement + LPS), FP (flaxseed supplement + PBS), and FL (flaxseed + LPS) for the supplement studies and DP (Dorset + PBS), DL (Dorset + LPS), SP (Suffolk + PBS), and SL (Suffolk + LPS) for the breed study. Ewes were synchronized for estrus with a 5-day controlled intravaginal drug releasing insert (CIDR) (Pfizer, New York, NY) followed by injection of $4 \mathrm{cc}$ of $\mathrm{PGF}_{2 \alpha}$ (Lutalyse ${ }^{\circledR}$, Pharmacia and UpJohn, New York, NY) at CIDR removal. Dorset and Suffolk ewes were always in separate pastures. With the supplement experiments, Dorset ewes were randomly divided into two groups on the day of CIDR removal for supplement feeding. A fertile ram wearing a marking harness was placed with each group on the day of CIDR removal, and marking by the ram was checked twice daily when ewes were fed. Day of the first breeding was denoted as day 0 post coitus (dpc). Ewes were weighed on the day of withdrawal of the CIDR, the day before the LPS challenge ( $\sim 7$ days on supplement), and at $25 \mathrm{dpc}$ ( 27days on supplement); supplement s did not affect ewe weights. All ewes were housed at the WVU Stewartstown Farm (WVU IACUC \# 13-0404.3).

The day before the experiment (supplement started 7 days prior), ewes were weighed, sorted into pens in the barn and given hay and water. Ewes were randomly assigned to receive either LPS $(2.5 \mu \mathrm{g} / \mathrm{kg})$ or PBS ( $3 \mathrm{ml}$ ) on day 5/6 post coitus (dpc). At 8 am, a blood sample was collected before jugular administration of LPS or PBS. This dose of LPS has been used in previous experiments (Elsasser et al, 2004; Graham, 2014; Nikpour, 2017). Jugular blood samples ( $~ 8 \mathrm{ml})$ were collected via venipuncture into EDTA treated tubes and stored in coolers with ice packs until transported to the laboratory. In the spring, samples were collected at $0.5,1,1.5,2,2.5,3,4,5,6,7,8,9,10,11,12$, and 24 hours after LPS or PBS injection. During the fall, samples were collected at $0.25,0.5,0.75,1,1.5,2,3,4,5,6,9,12$, and 24 hours after LPS or PBS injection. Rectal temperature was recorded right before treatment administration 
and at each whole hour blood collection. Observable changes in behavior and mucosal responses after treatment (lethargy, coughing, vaginal discharge, nasal discharge, and inappetance) were recorded at each blood collection. After the 24 hour sample, ewes were returned to pasture. Additional blood samples were collected via venipuncture into EDTA treated tubes on 8/9, 15/16 and 25/26 dpc.

White blood cell counts in $20 \mu 1$ of whole blood collected at the whole hour time points were obtained using a Beckman Coulter Counter (Beckman Coulter, Pasadena, CA). Samples were then centrifuged at $2500 \mathrm{rpm}$ for 20 minutes, and plasma was collected and stored at -80 . In the spring supplement study, buffy coats were collected and pooled for each group at each time point (Flax + LPS, Flax + PBS, Con + LPS, Con + PBS). In the fall, buffy coats were collected, and 2-3 samples at each time point were randomly pooled for ewes recieving the same supplement and treatment giving 4 pooled samples for each supplement with each treatment. For the breed study, buffy coats were collected, and 4 samples were randomly pooled within treatment for the Suffolk ewes and 2 to 3 samples were randomly pooled within treatment for the Dorset ewes giving 4 pooled samples for each breed with each treatment at each time point. Cells in the buffy coat were re-suspended in PBS, and any remaining red blood cells were removed using ACK lysis buffer (Lonza catalog \#10-584E) and washed with PBS. Buffy coat was transferred to a polypropylene tube and homogenized in $1.5 \mathrm{ml}$ RNA-bee (Tel-Test, Friendswood, TX) and stored at -80 until RNA extraction.

Detection for pregnancy occurred 25 or $26 \mathrm{dpc}$ via transrectal ultrasonography with a $7.5 \mathrm{MHz}$ transducer and Aloka 500 ultrasound console (Corometrics Medical Systems, Inc.Wallingford, CT) for visualization of an embryonic heartbeat. Date of birth, number of lambs born and lamb sex were recorded after lambing. 


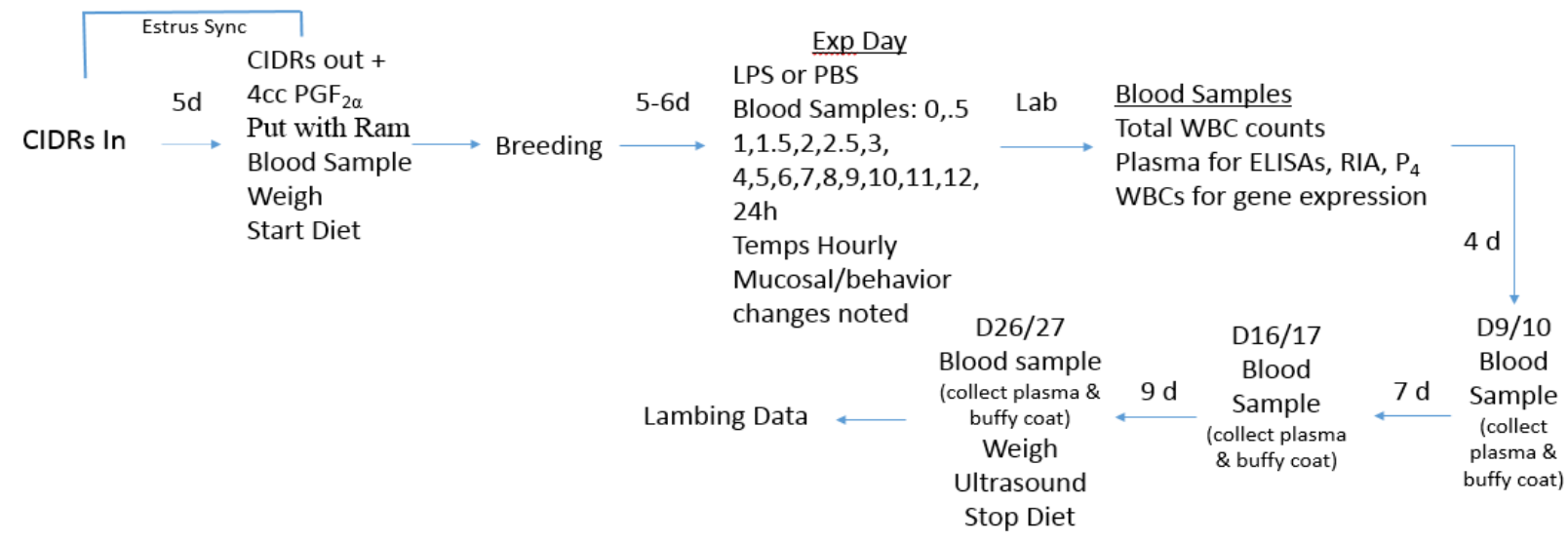

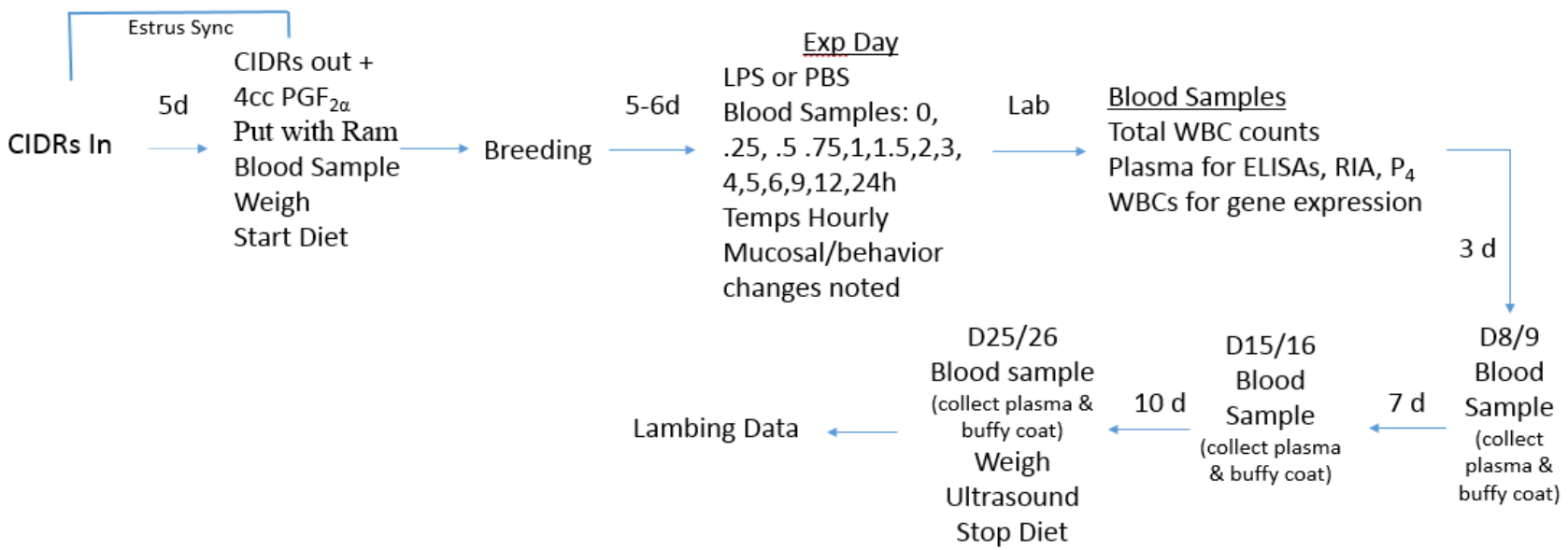

Figure 1: Timeline outlining the steps of trial 1 (top) of the omega-3 PUFA study (Spring 2015) and the experiments that occurred in Fall 2015 (trial 2 of the omega-3 PUFA study and breed study) (bottom) The only major difference is the blood collection times on the experimental day.

\section{Feed Manufacture}

Feed was prepared in weekly batches at West Virginia University's Pilot Feed Mill using a Davis horizontal ribbon mixer (Bonner Springs, KS). The flaxseed supplement consisted of $45 \%$ sheep ration and 55\% whole flaxseed (Table 2 and 6) (Commodity Marketing Company, Canada) purchased from Valley Point Feed (Albright, WV; Trial 1: Lot \# DM1415; Trial 2: Lot \# 15-0072), and ingredients for the control supplement were stored at the Pilot Feed Mill. The control supplement consisted of 45\% sheep ration and a 55\% mixture of cracked corn, soybean meal, and soybean oil (Table 2 and 6) formulated to match the fat and protein in the flaxseed (Tables 1 and 5). All components of the flaxseed supplement were added to the mixer and allowed to mix for at least 5 minutes before being transferred to feed bags for storage. All components of the control supplement except the soybean oil were added to the mixer and 
mixed for $5 \mathrm{~min}$. The soybean oil was then added, and the supplement was mixed for an additional 5 minutes. Control supplement was transferred to a plastic storage bin. Both supplement s were stored in the feed mill and weighed out in buckets to take to the ewes. Ewes were kept on pasture, had access to water ad libitum, and were randomly assigned to receive $0.24 \mathrm{~kg}$ per head twice a day of the respective supplement beginning the afternoon CIDRs were removed until 25/26 dpc.

Samples of both supplements were analyzed (Tables 3, Tables 7) by the Rumen Fermentation Profiling Laboratory at WVU. Ten attributes were compared between samples of the prepared flaxseed and control supplement s (Table 5, Figure 10, Table 6, Figure 20). Attributes were compared by T-tests and Bonferroni correction was used to control for multiple comparisons (10 attributes) between the supplement $\mathrm{s}$; differences with $\mathrm{p}<0.005$ were considered statistically significant. In both trials, the flaxseed supplement had a higher percentage of neutral detergent fiber (trial 1: control: $6.77 \pm 0.28$, flaxseed: $19.70 \pm 0.78, \mathrm{p}=0.0001$; trial 2: control: $10.48 \pm 0.86$, flaxseed: $21.39 \pm 1.85, \mathrm{p}=0.0002$,) and a lower percentage of non-fiber carbohydrate (trial 1: control: $41.58 \pm 0.90$, flaxseed: $33.39 \pm 0.67$, $\mathrm{p}=0.0005$; trial 2: control: $42.46 \pm 0.89$, flaxseed: $32.84 \pm 1.33, \mathrm{p}<0.0001)$.

\section{Gene Expression}

For RNA extraction, frozen homogenates (white blood cells in RNA Bee, Fisher Scientific, Waltham, MA) were thawed for $\sim 5$ minutes in a $37^{\circ} \mathrm{C}$ water bath; $150 \mu \mathrm{L} 24: 1$ chloroform/isoamyl (Sigma-Aldrich, St. Louis, MO) was added to each tube, which was then shaken vigorously for 15 seconds and transferred to a microcentrifuge tube. Samples sat on ice for 5 minutes before being centrifuged for 15 minutes at $4^{\circ} \mathrm{C}$ and $14,000 \mathrm{x}$ g. Following centrifugation, the aqueous (top) layer was transferred to a new microcentrifuge tube, in which RNA was precipitated by the addition of an equal volume of ice cold isopropanol to the sample, gently inverting the tube to mix the contents and incubating on ice for 15 minutes. Samples were then centrifuged for 15 minutes at $14,000 \mathrm{x} g$ at $4^{\circ} \mathrm{C}$ resulting in the formation of an RNA pellet. The RNA was washed twice with $1 \mathrm{ml}$ 75\% cold ethanol (centrifuged $10 \mathrm{~min}$ at $14,000 \mathrm{x}$ g at $4^{\circ} \mathrm{C}$ ), dried under a vacuum and dissolved in $\sim 20 \mu 1$ ultrapure water. Quality and quantity of RNA was assessed using Take3 microvolume plate (BioTek, Winooski, VT), and samples with an $\mathrm{OD}_{260} / \mathrm{OD}_{280}$ ratio $>1.8$ were accepted.

Extracted RNA was diluted in $13 \mu \mathrm{l}$ of DEPC-treated water to a total concentration of $1.8 \mu \mathrm{g}$, and cDNA synthesis was conducted by making a master mix composed of $2.5 \mu \mathrm{l}$ of $2.5 \mathrm{mM}$ dNTP (GE Illustra, Buckinghamshire, UK), $5.0 \mu \mathrm{l} 5 \mathrm{X}$ reverse transcriptase buffer (Life Technologies, Burlington, ONT Canada), $2.0 \mu \mathrm{l}$ 0.01 M Dithiothreitol (DTT) (Life Technologies, Burlington, ONT Canada), $0.5 \mu \mathrm{l}$ RNasin (Promega, Madison, WI), and $2.0 \mu \mathrm{l}$ random hexamer primers (Life Technologies, Burlington, ONT Canada). Master mix $(12 \mu \mathrm{l})$ was added to each sample $(13 \mu \mathrm{l})$ and then transferred to a 96 well 
plate. Using a T100 thermal cycler (Bio-Rad, Hercules, CA) samples were denatured at $70^{\circ} \mathrm{C}$ for 5 minutes and then chilled at $4^{\circ} \mathrm{C}$ for 5 minutes. SuperScript ${ }^{\mathrm{TM}} \mathrm{II}(1 \mu \mathrm{l})$ (Life Technologies, Burlington, ONT Canada) was then added to each sample. Reverse transcription reaction was conducted by heating to $37^{\circ} \mathrm{C}$ for 60 minutes, $90^{\circ} \mathrm{C}$ for 5 minutes, and cooling to $4^{\circ} \mathrm{C}$ for 5 minutes using a T100 thermal cycler (BioRad, Hercules, CA). Quantity of cDNA was determined using Take3 microvolume plate (BioTek, Winooski, VT). For trial 1 of the supplement study, samples were diluted 1:5 in DEPC water. For the Fall experiments, samples were diluted to $4 \mathrm{ng} / \mu \mathrm{l}$ in DEPC water. The RNA was stored at $-20^{\circ} \mathrm{C}$.

Primer design was based on mRNA sequences found in the NCBI database. For each candidate gene, primers were selected using Primer3 software (Rozen and Skaletsky, 2000). Changes to the default program settings included product range $80-120 \mathrm{bp}$, primer $T_{m} 59-61^{\circ} \mathrm{C}$, primer GC\% 40-60, max selfcomplementarity 3, max 3' self-complementarity 1, and max poly-x 3. Primers were purchased from Integrated DNA Technologies (Coralville, Iowa); associated GenBank accession numbers and primer sequences used in qPCR are shown in Table 4. All primers were used previously (Sommers, 2014; Jacobs et al 2016) except NF- $\mathrm{B}$ and SOD2 (Chauhan et al, 2014). The primer efficiency was derived (Sommers, 2014) from the slope of the regression line fitted to a subset of baseline-corrected data points in the loglinear phase using LinRegPCR (Ramakers et al, 2003). Efficiency for each primer was higher than (1+E) $=1.96$. The PCR products were visualized after agarose gel electrophoresis to confirm amplification of only one product when the primers were first purchased (Sommers, 2014). Melting curve analysis resulted in one well defined peak per reaction indicating only one amplified PCR product. Melting temperatures (Tm) of products were consistent with theoretical Tm of the expected PCR products. Gene names and primer sequences are in Table 4.

Quantitative PCR reactions were conducted using a Bio-Rad CFX96 system (Bio-Rad, Hercules, CA). Samples were analyzed in duplicate, and the reaction was performed in a total volume of $20 \mu \mathrm{l}$ consisting of $10 \mu \mathrm{l} \mathrm{SYBR}$ Green ${ }^{\mathrm{TM}}$ Select Master Mix (Life Technologies, Burlington, ONT Canada), 1.2 $\mu 1$ forward primer, $1.2 \mu 1$ reverse primer, $5.6 \mu \mathrm{l}$ DEPC-treated water, and $2 \mu 1$ diluted template cDNA. The conditions for the $\mathrm{qPCR}$ reaction were $50^{\circ} \mathrm{C}$ for 10 minutes followed by initial denaturation at $95^{\circ} \mathrm{C}$ for 5 minutes, then 39 cycles of denaturation at $95^{\circ} \mathrm{C}$ for 10 seconds, and extension for 30 seconds at $60^{\circ} \mathrm{C}$. Relative fold changes in expression of candidate genes were obtained using the $2^{-\Delta \Delta \mathrm{Ct}}$ method (Livak and Schmittgen, 2001). The obtained $\mathrm{Ct}$ values were used to calculate $\Delta \mathrm{Ct}$ values of the genes of interest [Ct (test)-Ct (reference)]. The reference gene used for normalization was GAPDH. For trial 1 of the supplement study, the $\Delta \Delta \mathrm{Ct}$ values were obtained in reference to the control supplement + PBS group sample at each time point. For the fall experiments, the $\Delta \Delta \mathrm{Ct}$ values were obtained in reference to the sample collected at CIDR removal. Graphical representation for each gene is based on fold changes. 


\section{Assays}

Progesterone concentrations were measured in $25 \mu$ of plasma using an Immulite ${ }^{\circledR} 1000$ Immunoassay System, a solid phase, competitive immunoassay using enzyme-labeled chemiluminescent technology. Reportable range of the assay was $0.20-40 \mathrm{ng} / \mathrm{mL}$ with a sensitivity of $0.46 \mathrm{ng} / \mathrm{mL}$. Control samples were from an ovariectomized ewe (negative control) and an ovariectomized ewe injected with progesterone (positive control). For trial 1, P4 concentrations were measured in plasma collected at hour 0 on 5/6 dpc, 9/10 dpc, and 25/26 dpc. For the fall experiments, P4 concentrations were measured in plasma collected on days $8 / 9$ and 25/26 dpc.

Plasma samples were pooled to measure cortisol, Hp, and SAA. For trial 1, plasma samples were pooled for the time points $0,0.5,1,1.5,2,2.5,3,6,9,12$, and 24 hours post treatment among ewes receiving the same supplement and treatment at each time point. For trial 2, plasma samples were randomly pooled in groups of 2 to 3 samples within supplement and treatment (same groups as pooled buffy coat for gene expression) giving 4 pooled samples for each supplement with each treatment at the time points $0,1,2,3,6,9$, and 12 hours post treatment for cortisol and at the time points $0,2,6,12$, and 24 hours post treatment for Hp and SAA. For the breed study, plasma samples were randomly pooled in groups of 4 samples within treatment for the Suffolk ewes and 2-3 samples within treatment for Dorset ewes (same groups as pooled buffy coat for gene expression) giving 4 pooled samples for each breed with each treatment at the time points $0,1,2,3,6,9$, and 12 hours post treatment for cortisol and at the time points $0,2,6,12$, and 24 hours post treatment for Hp and SAA.

Concentrations of cortisol were measured using a solid phase competitive ELISA kit from Immuno-Biological Laboratories, Inc (Minneapolis, MN; Catalog \# IB79135) following manufacturer's instructions. The assay had a detection range of $0-800 \mathrm{ng} / \mathrm{mL}$ with a sensitivity of $2.5 \mathrm{ng} / \mathrm{mL}$ and an intraand inter-assay CV of $4.73 \%$ and $11.78 \%$ respectively. Absorbance was read at $450 \mathrm{~nm}$ and raw absorbance values for samples and standards were adjusted for blank absorbance. SAA was measured using a sheep quantitative sandwich ELISA kit from mybiosource (San Diego, CA; Catalog \# MBS024559) following manufacturer's instructions. The assay had a detection range of 3.12-100 $\mu \mathrm{g} / \mathrm{mL}$, a sensitivity of $1.0 \mu \mathrm{g} / \mathrm{mL}$, and intra- and inter-assay $\mathrm{CV}$ of $3.83 \%$ and $5.35 \%$ respectively. Absorbance was read at 450nm. Hp was measured using a sheep double antibody sandwich ELISA kit from mybiosource (San Diego, CA; Catalog \# MBS564186) following manufacturer's instructions. The assay had a detection range of $0-1000 \mathrm{ng} / \mathrm{mL}$ with a sensitivity of $15.63 \mathrm{ng} / \mathrm{mL}$ and an intra- and inter-assay CV of $3.51 \%$ and $16.41 \%$ respectively. Absorbance was read at $450 \mathrm{~nm}$. Hp was analyzed in samples diluted 1:2000 ( $2 \mu \mathrm{l}$ pooled sample into $198 \mu \mathrm{l}$ diluent then $15 \mu \mathrm{l}$ of diluted pooled sample into $285 \mu \mathrm{l}$ diluent) and reported as $\mathrm{mg} / \mathrm{mL}$. 


\section{Statistics}

Data were analyzed using JMP and SAS software (JMP®, Version Pro 12, Copyright $@ 2015$; SAS ${ }^{2}$, Version 9.3, SAS Institute., Cary, NC, Copyright (C) 2002-2010). Data that were not normally distributed were log transformed before analysis and significance criterion alpha level was 0.05 . Hourly responses in rectal temperature, hourly change in temperature from hour 0 , and white blood cells were analyzed by repeated measures ANOVA. Main effects in the supplement experiments included supplement (flaxseed or control), treatment (LPS or PBS), hour, all 2-way interactions and the 3-way interaction. Main effects in the breed study included breed (Dorset or Suffolk), treatment (LPS or PBS), hour, all 2-way interactions and the 3-way interaction. All results reported as mean \pm SEM.

For trial 1 of the supplement study, cortisol, Hp, SAA, and gene expression (CXCL8, TLR4, NFкB, IL-6, TNFa, IFNץ, COX2, C3, MRC1, IL-10, PPAR $\gamma$, FOXP3, SOD2) were measured in samples collected at various time points, but samples were pooled within supplement and treatment giving only one sample per group per time point. Therefore, time points served as trials for each group, and means were analyzed by ANOVA for the main effects of supplement, treatment, and their interaction. The Benjamini-Hochberg procedure was used to control the false discovery rate (FDR $=0.10$ ) with regard to gene expression.

For the Fall experiments, Hp, SAA, cortisol, and fold change in gene expression (CXCL8, TLR4, NF-kB, IL-6, TNF $\alpha$, IFN $\gamma$, COX2, C3, MRC1, IL-10, PPAR $\gamma$, FOXP3, TGF $\beta$, SOD2) were analyzed by repeated measures with irregular spacing ANOVA. When significant interactions were detected, least square means comparisons were made by slicing with the effect Supplement *Treatment*Hour, slicing supplement and hour (supplement study trial 2) or with the effect Breed*Treatment*Hour slicing breed and hour (breed study). The Benjamini-Hochberg procedure was used to control the false discovery rate $(\mathrm{FDR}=0.10)$ with regards to gene expression (CXCL8, TLR4, NF-kB, IL-6, TNFa, IFN $\gamma, \mathrm{COX} 2, \mathrm{C} 3$, MRC1, IL-10, PPARy, FOXP3, TGF $\beta$, SOD2).

For mucosal responses and noticeable behavioral changes, the number of symptoms recorded for each ewe was summed over time. Sickness symptoms, plasma progesterone at one time point, and the number of lambs were analyzed by two-way ANOVA and Tukey's HSD for the effects of breed (Dorset or Suffolk) or supplement (Flaxseed or Control) and treatment (LPS, PBS) and their interactions. In trial 1, a T-test was used to compare plasma progesterone between the supplement $\mathrm{s}$.

For the Fall experiments, using the average gestation length (148 days) and the lambing records retrospectively, conception date was determined. For each ewe it was then decided based on that determined mating date if she was pregnant with that lamb(s) during the experiment and thus did not 
experience embryonic loss or had a lamb from a mating that occurred after the experiment and could have experienced embryonic loss.

For trial 2 of the supplement study, multiple contingency analyses with Pearson's Chi-square were used to examine the possible association between 1) pregnancy diagnosis by ultrasound and treatment group (CP, CL, FP, FL); 2) pregnancy diagnosis by ultrasound and treatment (LPS, PBS); 3) pregnancy diagnosis by ultrasound and supplement (flaxseed, control); 4) a ewe lambing as a result of the mating that occurred before the experiment (no embryonic loss) and treatment (LPS, PBS); and 5) a ewe lambing as a result of the mating that occurred during the experiment and supplement (flaxseed, control). Logistic regression was conducted to examine if receiving LPS, eating flaxseed, or the interaction could predict the odds of a ewe having a lamb as a result of the mating that occurred before the experiment. For the breed study, multiple contingency analyses with Pearson's Chi square were used to examine possible associations between 1) pregnancy diagnosis by ultrasound and treatment group (DP, DL, SP, SL); 2) pregnancy diagnosis by ultrasound and breed (Dorset, Suffolk); 3) the association between a ewe having a lamb as a result of the mating that occurred during the experiment (no embryonic loss) and experimental group (DP, DL, SP, SL); 4) the association between a ewe having a lamb as a result of the mating that occurred before the experiment and breed (Dorset, Suffolk) and 5) the association between a ewe having a lamb as a result of the mating that occurred before the experiment and treatment (LPS, PBS). Logistic regression was conducted to examine if receiving LPS, breed, or their interaction could predict the odds of a ewe having a lamb as a result of the mating that occurred before the experiment.

\section{Chapter 3: Effects of Supplementing with Omega-3 Polyunsaturated Fatty Acids on LPS Challenge in Early Pregnant Dorset Ewes}

\section{Introduction}

Reproductive tract infections or immune responses outside of the reproductive tract can compromise fertility (Hansen, 2011a). Cows with clinical or subclinical mastitis are less likely to become pregnant and are more likely to experience pregnancy loss compared to healthy cows (Hansen et al., 2004). Immune responses to bacterial infections include the release of bacterial products, ROS, cytokines, and chemokines at the inflammation site that can travel systemically to act on the liver, hypothalamus, pituitary, and reproductive tract resulting in impaired fertility (Hansen, 2011a). This immune response can be mimicked in early pregnant sheep by systemically giving peptidoglycan-polysaccharide complex (Holásková et al., 2004; Dow et al., 2010) or LPS (Graham, 2014).

Several cytokines, including pro-inflammatory ones, are critical for establishing and maintaining early pregnancy and implantation. However, temporal-spatial secretion of these molecules is critical. Any insult, like an infection, that alters the temporal-spatial secretion of cytokines could negatively affect 
reproduction. Binding of PAMPs to PRRs activates intracellular signaling (MAPKs, AP-1; NF- $\mathrm{B}$ ) that leads to transcription/secretion of PICs (IL-1 $\beta$, IL-6, and TNF $\alpha$ ), chemokines (CXCL8), PGs, and antimicrobial peptides (Taakeuchi and Akira, 2010; Norris and Dennis, 2012; Badinga et al., 2016). Indeed, when Zarrin et al. (2014) injected $200 \mu$ of LPS, a gram negative bacteria PAMP, into udders of cows, gene expression of IL-1 $\beta$, IL-6, CXCL-8, IL-10, iNOS, TNF $\alpha, \mathrm{NF}-\kappa \mathrm{B}, \mathrm{Hp}$, and SAA increased in the tissue.

Microbial infections evoke inflammatory responses mediated by the infiltration of neutrophils, phagocytes and other innate immune cells, and the production of cytokines and chemokines to eliminate the infection before removing debris and resolving the inflammation. An infection that is not cleared, as might occur in a post-partum dairy cow in NEB with a suppressed immune system, can lead to chronic inflammation and damage of endometrial cells. Early responses of the uterus to pathogens involves neutrophilic influx into the superficial endometrium and then into the lumen (Bondurant, 1999). Neutrophils phagocytize bacteria with the aid of opsonins in the uterine fluid and attack the pathogens by releasing toxic substances (ROS, reactive nitrogen species, proteinase 3, cathepsin G, elastase) (Cheville, 1988; Nathan, 2006). These effector molecules are not able to distinguish between host and microbial targets, so damage to host tissue also occurs (Nathan, 2002). Besides neutrophils, other immune cells that will be at the site of invading pathogens include macrophages, eosinophils, mast cells, and lymphocytes (Bondurant, 1999). Activation of mast cells results in degranulation and release of chemotactic factors for neutrophils and eosinophils, PIMs (histamine, cytokines, PGs) (Bondurant, 1999), proteases that can activate the complement components C3 and C5 (Kéuther et al.,1998), and vasoactive substances (Kéuther et al.,1998) that increase the permeability of small vessels allowing serum (containing complement components and immunoglobulins) to leak into the superficial endometrial tissue and uterine lumen (Bondurant, 1999). Damage to endometrial epithelial cells from PIMs will cause release of IL-1 $\alpha$ (a damage associated molecular pattern (DAMP)), further increasing the inflammatory response (Medzhitov, 2008; Carneiro et al., 2016). Other DAMPs released from damaged or dead cells include ATP and nucleic acids that are thought to bind PRRs and initiate an inflammatory response (Chen and Nunez, 2010).

Chronic infection/inflammation in the endometrium will alter endocrine functions and can affect transport of spermatozoa and the hormonal and cytokine milieu involved in implantation of the blastocyst. Hill and Gilbert (2008) reported a reduction in trophoblast cells of bovine embryos cultured in media conditioned with endometrial fluid collected from an inflamed endometrium. The trophoblast is the outer layer of cells of the blastocyst that will be the fetal contribution to the placenta, and a reduction in the number of trophoblast cells will have a negative impact on implantation, placentation, and embryonic 
survival. Endometrial cytokines produced during infection/inflammation may alter steroidogenesis in luteal cells resulting in a lower secretion of progesterone than is necessary for pregnancy (Thatcher et al., 2010). The PICs (TNF $\alpha$, IL-1, and IFN $\gamma$ ) could act directly on the CL or indirectly hamper P4 production through the induction of $\mathrm{PGF}_{2 \alpha}$ production (Pate, 1995; Niswender et al., 2000; Petroff et al., 2001; Erlebacher et al., 2004).

Cell membranes are composed of phospholipids, in which PUFA are key structural and functional components (Calder, 2013). Phospholipids of cells involved in inflammation tend to have more AA than $\omega$-3 PUFA (Peterson et al., 1998; Healy et al., 2000), but the addition of fish oil (high in $\omega-3$ PUFAs) to the supplement of animals (Peterson et al., 1998; Mattos et al., 2004) or human volunteers (Rees et al., 2006) resulted in an increase in EPA and DHA in cell membranes with a reduction in AA content in the phospholipid pool. Norris and Dennis (2012) also reported that EPA supplementation to macrophages in vitro increased membrane EPA and DPA while DHA supplementation increased membrane DHA levels.

In vivo studies have shown that $\omega-3$ PUFAs decrease eicosanoid synthesis in the bovine uterus (Mattos et al., 2004; Petit et al., 2004) while in vitro studies have shown that exogenous EPA inhibits $\mathrm{PGF}_{2 \alpha}$ production by cultured bovine endometrial cells (Mattos et al., 2003; Caldari-Torres et al., 2006; Badinga et al., 2016). Effects of supplemental $\omega-3$ PUFA could be due to a decrease in AA in cell membranes and an increase in fatty acids that compete with AA for enzyme usage (Mattos et al., 2004).

In homeostatic conditions, $\mathrm{PLA}_{2}$ is mostly inactive, but, during infection or inflammation, PRRs can activate $\mathrm{PLA}_{2}$ causing it to translocate to the perinuclear and endoplasmic reticular membranes. At the membranes, $\mathrm{PLA}_{2}$ can release lipids to be used as substrates for other lipid mediators. PLA 2 can hydrolyze AA containing phospholipids releasing AA to be converted into pro-inflammatory eicosanoids by COX enzymes, or PLA 2 can release phospholipid-esterified $\omega$-3 PUFAs (EPA, DPA, and DHA), which can be turned into anti-inflammatory SPMs by COX and LOX enzymes working together (Norris and Dennis, 2012; Dennis and Norris, 2015). The $\omega-3$ PUFAs, are made from ALA; therefore, a supplement high in ALA (like flaxseed) potentially provides an individual with more SPMs during an inflammatory response and the ability to recover from that response faster.

When the macrophages in Norris and Dennis's study (2012) were stimulated with a TLR4 agonist or ATP, the media contained increased levels of the PUFAs that the macrophages were cultured with EPA or DHA showing that $\omega$-3 PUFA supplementation results in the FAs being incorporated into cell membranes, which are then available for use in the eicosanoid pathways. The macrophages then used the released FAs to produce COX metabolites. Cells supplemented with EPA or DHA produced lower levels of AA derived COX-metabolites $\left(\mathrm{PGE}_{2}, \mathrm{PGD}_{2}\right)$ and higher amounts of EPA-derived COX metabolites 
( $\mathrm{PGD}_{3}, \mathrm{PGE}_{3}$, and 5-HEPE) and DHA-derived COX metabolites, although COX-2 expression was not changed by supplementation. While AA COX-metabolites decreased with supplementation, 5-LOX AA metabolites (5-HETE) increased; 5-HEPE, the EPA version of 5-HETE, increased with EPA supplementation, and DHA was metabolized by 5-LOX to 4-HDoHE and 7-HDoHE, suggesting that the (-3 PUFA inhibited the COX pathway and shunted the FAs to the LOX pathway (Norris and Dennis, 2012). End products of the LOX pathway have anti-inflammatory effects.

ATP activates P2X receptor cation channels, which leads to increases in intracellular $\mathrm{Ca}^{2+}$ and translocation of $\mathrm{cPLA}_{2}$ to cell membranes to liberate stored phospholipids. Signaling through this receptor allows both AA and EPA to be utilized. However, activation of cPLA 2 through TLR4 does not result in release of non-AA PUFAs. Long-term TLR4 activation leads to $\mathrm{SPA}_{2}$ activation, which then contributes to eicosanoid production during late-phase activation, which will release $\omega$-3 PUFAs (Norris and Dennis, 2012). This suggests that the beginning of a response is set up to be pro-inflammatory with predominantly AA being released and utilized for eicosanoid production followed by release of $\omega-3$ PUFA causing a switch to resolution through inhibition of COX, shunting substrates from the COX to the LOX pathway, and serving as substrates for SPMs. Supplementation with $\omega-3$ PUFA and subsequent incorporation into cellular membranes can, therefore result in a stronger and quicker resolution phase.

Both EPA and AA are substrates for the PGHS enzymes, and the two fatty acids compete with each other for the enzymes. Thus, increasing the amount of available EPA in the membrane should result in increased synthesis of prostanoids of the 3 series with a decrease in the amount of AA being turned into prostanoids of the 2 series (Mattos et al 2003; Rees et al., 2006). Another explanation for effects of EPA may be that EPA reduces the activity or expression of phospholipase $\mathrm{A} 2, \mathrm{COX}$, and/or prostaglandin $\mathrm{H}$ synthase (PGHS) genes (Bousserouel et al., 2003; Wada et al., 2007) leading to reduced synthesis of $\mathrm{PGE}_{2}$ and $\mathrm{PGF}_{2 \alpha}$. Suppression of secretion of luteolytic $\mathrm{PGF}_{2 \alpha}$ is necessary for maintenance of the CL and progesterone production, which is required for establishment of pregnancy (Bazer et al., 2010).

Omega-3 PUFAs inhibit PIC production by preventing the phosphorylation and degradation of

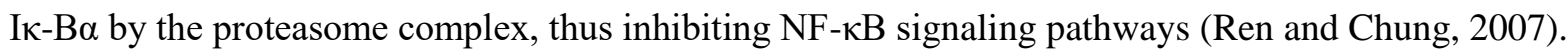
Both EPA and DHA inhibited IL-1 $\beta$, TNF- $\alpha$, and IFN $\gamma$ production by human lymphocytes in vitro (Purasiri et al., 1997), perhaps through inhibition of NF-kB signaling.

Flaxseed contains high amounts of the $\omega$-3 PUFA $\alpha$-linolenic acid (18:3 n-3), which can be converted by animal tissues to EPA and DHA. The objective of the following two studies was to examine if providing supplementary $\omega-3$ PUFAs in the form of whole flaxseed to ewes would have beneficial 
effects on the inflammatory response to an LPS challenge during early pregnancy. Reducing the inflammatory response to bacterial infections could help mitigate early embryonic loss in dairy cows.

\section{Results}

\section{Clinical Signs}

Temperature

Treatment with LPS $(\mathrm{p}<0.001)$ increased rectal temperature that peaked at hour 4 before returning to normal $(\mathrm{p}<0.001)$. Ewes fed the flaxseed supplement had a blunted temperature increase in response to LPS, peaking at $40.05 \pm 0.26^{\circ} \mathrm{C}$ compared to the CL group which peaked at $41.03 \pm 0.14^{\circ} \mathrm{C}$. Temperatures in the FL group returned to normal by hour 6; the temperatures in the CL group did not return to normal until hour 8 ( $\mathrm{p}<0.0064$, Figure 2).

\section{Behavior changes/Mucosal Response}

Due to the low frequency of each individual sickness symptom, observed mucosal response (nasal discharge, vaginal discharge, coughing, and diarrhea) and behavioral change (loss of appetite, lethargic, lying down, and heavy breathing) were summed for each ewe over the 12 hours post treatment. Ewes treated with LPS $(\mathrm{p}<0.0001)$ displayed both mucosal responses and sickness behaviors, whereas no PBS ewes did, with the FL ewes averaging 8 symptoms and the CL ewes averaging 4 symptoms over the 12 hours. There was a tendency for effects of feed $(\mathrm{p}=0.0945)$ and feed $\mathrm{x}$ LPS interaction $(\mathrm{p}=0.0588$, Figure 3) to increase sickness symptoms.

\section{Immune System}

\section{White Blood Cell Counts}

Counts of white blood cells (WBCs) decreased between hour 0 and 1 in all groups and then returned to normal concentrations in the PBS groups by hour 2. The LPS $(\mathrm{p}<0.001)$ groups had a larger decrease in WBCs after treatment, and it took until hour 5 for the FL group and until hour 6 for the CL group to return to normal counts $(\mathrm{p}=0.0056)$. The more rapid return to normal WBC counts in the FL group resulted in a feed effect $(\mathrm{p}=0.0157)$ and a feed $\mathrm{x}$ LPS $\mathrm{x}$ hour effect $(\mathrm{p}=0.0056)$ (Figure $4 \mathrm{~b})$. White blood cells started to increase above the hour 0 values at hour 6 in the flaxseed groups and after hour 7 in the control supplement groups regardless of LPS or PBS ( $<<0.0001$, Figure 4a). From hour 7 to 12 the flaxseed groups had more WBCs than the control supplement groups $(\mathrm{p}<0.0001)$.

\section{Acute Phase Proteins}

Concentrations of Hp differed by feed $(\mathrm{p}<0.0001)$, LPS $(\mathrm{p}<0.0001)$ and feed $\mathrm{x}$ LPS $(\mathrm{p}<0.0001)$ with the FL group having the highest concentration of Hp $(0.68 \pm 012 \mathrm{mg} / \mathrm{ml})$. The CL $(0.14 \pm 0.01$ $\mathrm{mg} / \mathrm{ml}$ ) group had increased Hp counts compared to PBS (FP $0.04 \pm 0.00 \mathrm{mg} / \mathrm{ml}, \mathrm{CP}: 0.04 \pm 0.00 \mathrm{mg} / \mathrm{ml}$ ) groups, but not as elevated as the FL group (Figure 5a). Plasma concentratinos of SAA were affected by feed $(\mathrm{p}=0.02116)$, LPS $(\mathrm{p}<0.0001)$, and feed $\mathrm{x}$ LPS $(\mathrm{p}<0.001)$ with the CL $(1.86 \pm 0.22 \mu \mathrm{g} / \mathrm{ml}))$ group 
having lower SAA levels than the other 3 groups (FL $4.67 \pm 0.44 \mu \mathrm{g} / \mathrm{ml}, \mathrm{FP} 4.83 \pm 0.6 \mu \mathrm{g} / \mathrm{ml}, \mathrm{CP} 5.82 \pm$ $0.23 \mu \mathrm{g} / \mathrm{ml}$, Figure $5 \mathrm{~b}$ ).

\section{Cortisol}

Plasma cortisol levels were affected by feed $(\mathrm{p}=0.0468)$ and LPS $(\mathrm{p}<0.001)$. Lipopolysaccharide increased cortisol levels (LPS $124.76 \pm 12.96 \mathrm{ng} / \mathrm{ml}$, PBS $42.17 \pm 7.77 \mathrm{ng} / \mathrm{ml}$ ) and ewes on the flaxseed supplement had lower cortisol levels (Flaxseed $67.67 \pm 12.5 \mathrm{ng} / \mathrm{ml}$, Control $99.26 \pm 15.01 \mathrm{ng} / \mathrm{ml}$, Figure $6)$.

\section{Gene Expression}

Fold change in gene expression of CXCL8 was affected by LPS ( $\mathrm{p}<0.0001)$ and feed $(\mathrm{p}=0.0486)$ with LPS increasing CXCL8 (LPS 7.32 \pm 1.5 , PBS $1.1 \pm 0.19$ ) and the flaxseed supplement decreasing CXCL8 (Flaxseed $2.76 \pm 0.61$, Control $5.67 \pm 1.72$ ). There was a feed x LPS interaction on CXCL8 (FL $4.31 \pm 0.99$, FP $1.2 \pm 0.45$, CL $7.52 \pm 2.2, \mathrm{CP}: 1 \pm 0, \mathrm{p}=0.027$ ), such that the flaxseed supplement dampened the CXCL8 increase in response to LPS (Figure 7a). Expression of TLR4 was increased by LPS (LPS $4.6 \pm 0.77$, PBS $1.06 \pm 0.06, p<0.0001$ ) with flaxseed dampening the increase in response to LPS treatment ( $\mathrm{p}=0.0326)(\mathrm{CP} 1 \pm 0, \mathrm{CL} 6.1 \pm 3.73$, FP $1.13 \pm 0.32$, FL $3.11 \pm 1.15)$ (Figure $7 \mathrm{~d})$. No effects were found with regards to NF- $\kappa \mathrm{B}$ expression, but the CL group had the largest fold change (2.46 $\pm 1.15)$ followed by the FP group $(1.92 \pm 0.39)$ and then the FL group $(1.38 \pm 0.43)$ compared to the CP group (Table 8). IL-6 was affected by feed x LPS ( $\mathrm{p}=0.0296)$ with the FL group $(2.09 \pm 0.54)$ having a smaller fold changed compared to the FP $(2.82 \pm 0.69)$ and CL (2.62 \pm 0.63$)$ groups (Figure 7b). There was no differences in TNF $\alpha$ or COX2 (Table 8) expression among treatment groups. LPS $(\mathrm{p}=0.0024)$ decreased IFN $\gamma$ expression (LPS $0.58 \pm 0.14$, PBS: $1.26 \pm 0.23$ ) (Figure 7c). C3 increased in response to LPS (LPS $4.02 \pm 0.67$, PBS $1.79 \pm 0.32, \mathrm{p}=0.0065)$ with the CL group having the largest change (CP 1, CL 4.81 \pm 0.33 , FP $2.58 \pm 0.48$, FL 3.32 \pm 0.77 ) (Figure 7e). MRC1 decreased in response to LPS (LPS 0.34 \pm 0.07 , PBS $0.96 \pm 0.08, \mathrm{p}<0.0001)$ and fold change was similar between the FP $(0.92 \pm .15)$ and $\mathrm{CP}(1 \pm 0)$ groups and between the FL $(0.35 \pm 0.09)$ and CL $(0.33 \pm 0.12)$ groups (Figure $8 \mathrm{a})$. IL-10 was not affected by LPS, feed, or their interaction but FL $(2.82 \pm 0.96)$ had the greatest fold change followed by FP (2.07 $\pm 0.49)$ and then CL $(1.67 \pm 0.61)$ compared to the CP $(1 \pm 0)$ group (Table 8$)$. PPAR $\gamma$ was not affected by LPS, feed, or their interaction $(\mathrm{p}=0.0702)$ but the FL $(0.65 \pm 0.16)$ group did have a reduction in fold change compared to the other 3 groups (CP 1, CL $1.18 \pm 0.24$, FP $1.18 \pm 0.16$, Table 8). LPS decreased FOXP3 expression (LPS $0.73 \pm 0.2$, PBS $0.91 \pm 0.05$, p=0.0259, Figure 8c) and increased SOD2 expression (LPS $4.31 \pm 0.82$, PBS $1.00 \pm 0.08, \mathrm{p}<0.0001$, Figure $8 \mathrm{~b}$ ). 


\section{Reproductive System}

\section{Progesterone}

Progesterone concentrations were not among between the 4 treatment groups on 5/6 dpc (FL 1.74 $\pm 0.17 \mathrm{ng} / \mathrm{ml}$, FP $1.93 \pm 0.2 \mathrm{ng} / \mathrm{ml}$, CL $1.71 \pm 0.27 \mathrm{ng} / \mathrm{ml}$, CP $1.65 \pm 0.26 \mathrm{ng} / \mathrm{ml}$ ) or 25/26 dpc. When comparing all four groups, the flaxseed supplement increased P4 (CP $2.33 \pm 0.58 \mathrm{ng} / \mathrm{ml}$, CL $2.9 \pm 0.66$ ng/ml, FP $4.4 \pm 0.22$ ng/ml, FL $3.29 \pm 0.57$ ng/ml, p=0.0752) (Figure 9a). However, when comparing P4 concentrations on day 25/26 post coitus only between the supplement s (Flaxseed $3.84 \pm 0.37 \mathrm{ng} / \mathrm{ml}$, Control $2.62 \pm 0.43 \mathrm{ng} / \mathrm{ml}$ ), supplementary flaxseed increased P4 concentrations ( $\mathrm{p}=0.0481$ ) (Figure 9b).

\section{Trial 2 Supplement Study}

\section{Results}

\section{Clinical Signs}

\section{Temperature}

Ewes treated with LPS $(\mathrm{p}<0.0001)$ had an increase in rectal temperature that peaked at 4 hours $\left(\mathrm{CP} 39.23 \pm 0.18^{\circ} \mathrm{C}, \mathrm{CL} 39.89 \pm .29^{\circ} \mathrm{C}, \mathrm{FP} 38.87 \pm 0.1^{\circ} \mathrm{C}, \mathrm{FL} 40.56 \pm 0.29^{\circ} \mathrm{C}\right)$ post treatment before decreasing and approaching normal temperatures by hour $6(\mathrm{p}<0.0001$, Figure 12a). There was a flaxseed x LPS effect ( $\mathrm{p}=0.0164$ ); ewes fed the flaxseed supplement and given LPS had higher temperatures than the other 3 groups at hour $2(\mathrm{p}=0.009), 3(\mathrm{p}<0.0001), 4(\mathrm{p}<0.0001), 5(\mathrm{p}<0.001)$, and hour $6(\mathrm{p}=0.014)$ (Figure 12a). Comparing the average rectal temperature over the 6 hours post treatment, LPS $(\mathrm{p}<0.0001)$ caused an increase in temperature but more so in the flaxseed-fed ewes than in the control supplement fed ewes $\left(\mathrm{CP}: 39.29 \pm 0.07^{\circ} \mathrm{C}\right.$; CL: $39.59 \pm 0.11^{\circ} \mathrm{C}$; FP: $38.94 \pm 0.04^{\circ} \mathrm{C}$; FL: $39.94 \pm 0.1^{\circ} \mathrm{C}, \mathrm{p}<0.0001$, Figure 12b).

\section{Behavior changes/Mucosal Responses}

Due to the low frequency of each individual symptom, all observed mucosal responses and behavioral changes were summed for each ewe over the 12 hours post treatment. Administration of LPS caused ewes to have observable physiological changes (LPS $1.18 \pm 0.38$, PBS $0.15 \pm 0.11, \mathrm{p}<0.0001$ ). Neither feed nor its interaction with treatment affected display of symptoms (Figure 13).

\section{Immune System}

\section{White Blood Cell Counts}

Treatment with LPS ( $\mathrm{p}=0.0037$ ) resulted in a drop in white blood cells from hour 0 to 1 . Concentrations then returned to normal by hour 6 and continued to increase so that the LPS ewes had higher WBCs at hours 12 and 24 compared to the PBS ewes (Figure 14a). Examining the hours (hour 0-6) from the LPS $(\mathrm{p}<0.0001)$ induced drop in WBCs until recovery; there was an hour effect $(\mathrm{p}=0.0004)$ and a LPS $x$ hour $(p<0.0001)$ effect with the LPS groups having significantly lower WBCs at hour 1 
$(\mathrm{p}=0.0002), 2(\mathrm{p}=0.0002), 3(\mathrm{p}<0.0001), 4(\mathrm{p}<0.0001), 5(\mathrm{p}=0.019)$ (Figure 14b). There was no effect of supplement.

\section{Acute Phase Proteins}

Concentrations of Hp were affected by LPS $(p=0.0327)$, hour $(p=0.0078)$, and the interaction of LPS $x$ hour $(\mathrm{p}=0.0165)$ with concentrations of Hp increasing in the two LPS groups starting after hour 6 . Hp increased more in the FL group, giving them more Hp at hour 24 compared to the control supplement ewes that received LPS (FL: $0.15 \pm 0.01 \mathrm{mg} / \mathrm{ml}$; CL: $0.07 \pm 0.02 \mathrm{mg} / \mathrm{ml}, \mathrm{p}=0.0009$, Figure 15a). SAA was affected by hour ( $\mathrm{p}=0.0023$ ) and the FP group had different SAA concentrations compared to the CP group at hour 2 (FP: $34.84 \pm 8.17 \mu \mathrm{g} / \mathrm{ml}$; CP: $17.88 \pm 4.68 \mu \mathrm{g} / \mathrm{ml}, \mathrm{p}=0.0265)$ and hour $12(\mathrm{FP} 40.67 \pm$ $12.55 \mu \mathrm{g} / \mathrm{ml}, \mathrm{CP} 17.38 \pm 3.21 \mu \mathrm{g} / \mathrm{ml}, \mathrm{p}=0.0029$, Figure $15 \mathrm{~b})$.

\section{Cortisol}

Concentrations of plasma cortisol increased in response to LPS $(\mathrm{p}<0.0001)$ and were affected by feed $(p=0.0235)$, hour $(p<0.0001)$ and the interactions feed $x$ LPS $(p=0.0007)$, LPS $x$ hour $(p<0.0001)$, and feed $x$ LPS $x$ hour $(\mathrm{p}=0.0105$ ) (Figure 16). Cortisol started to increase in the FL group right after LPS administration, peaked at hour 1 before gradually decreasing back to normal by hour 12 . The control supplement ewes given LPS, however, had a spike in cortisol only at hour 3. These patterns (Figure 16) resulted in the FL group having more cortisol than the CL group at hour 1 (FL $129.67 \pm 11.3 \mathrm{ng} / \mathrm{ml}, \mathrm{CL}$ $24.61 \pm 12.31 \mathrm{ng} / \mathrm{ml}, \mathrm{p}<0.0001)$, hour $2(\mathrm{FL} 127.62 \pm 17.91 \mathrm{ng} / \mathrm{ml}$, CL $38.98 \pm 7.9 \mathrm{ng} / \mathrm{ml}, \mathrm{p}<0.0001)$, and hour 6 (FL $65.5 \pm 20.63 \mathrm{ng} / \mathrm{ml}$, CL $22.23 \pm 4.26 \mathrm{ng} / \mathrm{ml}, \mathrm{p}=0.013)$.

\section{Gene Expression}

Treatment with LPS ( $\mathrm{p}=0.016)$ upregulated expression of CXCL8. Expression in the CL ewes increased after treatment, peaked at hour $4(58.19 \pm 53.37)$, and then decreased to normal by hour 9 . The CL ewes had higher expression at hour 4 compared to the CP ewes (CL 58.19 \pm 53.37 , CP $1.98 \pm 0.55$, $\mathrm{p}=0.0004$ ). Expression in the FL ewes increased more gradually and peaked at hour 6 (FL 29.03 \pm 26.99 ) before going back to normal by hour 9 (Figure 17a). There was no significant effect of the flaxseed or any of its interactions with LPS.

LPS administration ( $\mathrm{p}=0.0002)$ upregulated TLR4 expression. In both LPS groups, expression increased after hour 1 and peaked at hour 3 before returning to normal by hour 9 (hour $\mathrm{p}<0.0001$; LPS $\mathrm{x}$ hour $\mathrm{p}<0.0001$ ). The FL group had greater expression compared to FP ewes at hour 3 (FL 12.82 \pm 1.73 , FP $2.3 \pm 0.29, \mathrm{p}<0.0001)$ and hour 6 (FL $8.04 \pm 0.97$, FP $0.98 \pm 0.18, \mathrm{p}<0.0001)$. The control ewes administered LPS had greater expression of TLR4 compared to PBS ewes at hour 3 (CL $9.57 \pm 2.78$, CP $3.4 \pm 0.86, \mathrm{p}<0.0001$, Figure 17c). 
There were no main effects or interactions that affected NF- $\mathrm{BB}, \mathrm{IL}-6, \mathrm{TNF} \alpha$, IFN $\gamma$, or IL-10 expression (Table 9). Expression of COX2 fluctuated with time ( $\mathrm{p}=0.0119)$, but there were no supplement or treatment effects (Figure 17d).

Expression of C3 was upregulated in response to LPS (LPS $1.01 \pm 0.13$, PBS $0.75 \pm 0.07$, $\mathrm{p}=0.0107$ ) and was affected by the feed (Flaxseed $1.03 \pm 0.12$, Control $0.74 \pm 0.08, \mathrm{p}=0.0041$ ), hour $(\mathrm{p}<0.0001)$, and the interaction of LPS $x$ hour $(\mathrm{p}<0.0001)$. The FP ewes had greater expression 15 minutes post treatment compared to the FL ewes (FP $1.38 \pm 0.82$, FL $0.41 \pm 0.11, \mathrm{p}=0.0246$ ) while the FL ewes had greater C3 expression at hour 3 (FP $0.70 \pm 0.3$, FL $2.97 \pm 0.78, \mathrm{p}<0.0001$ ) and at hour 6 (FP $0.78 \pm 0.39$, FL $3.11 \pm 0.62, \mathrm{p}<0.0001)$. Compared to the CP ewes, the CL ewes had greater C3 expression at hour 3 (CP $0.63 \pm 0.03$, CL $2.04 \pm 0.52$, p=0.0014) and hour 6 (CP $0.68 \pm 0.05$, CL $2.09 \pm$ $0.30, \mathrm{p}=0.0014$ ) (Figure 17b).

Administration of LPS downregulated MRC1 expression (LPS 0.61 \pm 0.16 , PBS 1.24 \pm 0.20 , $\mathrm{p}=0.0118)$, which was also affected by hour $(\mathrm{p}=0.0004)$ and the interaction of LPS $\mathrm{x}$ hour $(\mathrm{p}=0.0091)$. Expression decreased after LPS administration, reached its lowest expression at hour 12 and had started to go back up by hour 24. Compared to CP ewes, the CL ewes had significantly lower expression at hour 12 (CP 1.74 \pm 1.00 , CL $0.26 \pm 0.14, \mathrm{p}=0.0143$ ) (Figure 18a).

Expression of PPAR $\gamma$ was affected by LPS (LPS $0.79 \pm 0.10$, PBS $1.62 \pm 0.15, \mathrm{p}=0.0014$ ) and hour $(\mathrm{p}=0.0436)$. While expression in the two PBS groups fluctuated across time, expression change in the LPS groups had a pattern. PPARy expression increased in the CL group from hour 1 to 3, decreased until hour 9 and then stayed low. Expression in the FL group increased only gradually from hour 1 to 6 before decreasing and staying low. Compared to the $\mathrm{CP}$ group, the CL group had significantly lower PPAR $y$ expression at hour $6(\mathrm{CP} 2.78 \pm 0.93$, CL $0.96 \pm 0.36, \mathrm{p}=0.0085)$ (Figure 18c).

Expression of FOXP3 was affected by the interaction of LPS $x$ hour $(\mathrm{p}=0.0242)$. Expression in the FL group increased from hour 0 to 2 before decreasing until hour 12. Expression had resumed slightly by hour 24. Expression in the CL group fluctuated until hour 3 before decreasing at hour 6 and then gradually increasing back to normal by hour 24. Compared to the FP group, expression in the FL group was decreased at hour 12 (FP $1.43 \pm 0.62$, FL $0.10 \pm 0.06$, p=0.0208) and hour 24 (FP $1.59 \pm 0.48$, FL $0.33 \pm 0.12, \mathrm{p}=0.0268$ ). Compared to the $\mathrm{CP}$ group, the $\mathrm{CL}$ group had decreased expression at hour 12 (CP $1.72 \pm 0.47$, CL $0.57 \pm 0.67, \mathrm{p}=0.0441$ ) (Figure 18d).

Expression of TGF $\beta$ was affected by hour ( $\mathrm{p}=0.0117)$. The PBS groups gradually decreased in expression from hour 0 to hour 3, increased at hour 4, and then decreased in expression at hour 6 . The FL 
group gradually decreased in TGF $\beta$ expression from hour 0 until hour 9 before increasing expression at hour 12. The CL group just gradually decreased in expression from hour 0 until hour 12. Compared to the FP group, the FL group had lower expression at hour 4 (FP $1.85 \pm 0.32$, FL $0.97 \pm 0.54$, p=0.0363) and hour 9 (FP $1.28 \pm 0.06$, FL $0.34 \pm 0.08, \mathrm{p}=0.0132$ ). Compared to the CP group, the CL group had lower expression at hour 12 (CP $1.20 \pm 0.16, \mathrm{CL} 0.28 \pm 0.09, \mathrm{p}=0.015)$ (Figure 18e).

Expression of SOD2 was affected by LPS (LPS $5.93 \pm 0.81$, PBS $1.83 \pm 0.15, \mathrm{p}<0.0001$ ), hour $(\mathrm{p}<0.0001)$, and the interaction of LPS $x$ hour $(\mathrm{p}<0.0001)$. While SOD2 expression stayed level in the PBS groups it increased in the LPS groups from hour 0 to hour 3 before decreasing back to normal by hour 12. Compared to the FP group, the FL group had higher SOD2 expression at hour 1.5 (FP $3.14 \pm$ 1.09 , FL $8.1 \pm 2.11, \mathrm{p}=0.0413$ ), hour 3 (FP $2.14 \pm 0.51$, FL $18.35 \pm 7.60, \mathrm{p}<0.0001)$ and hour 6 (FP $1.24 \pm$ 0.08, FL $16.04 \pm 6.96, \mathrm{p}<0.0001)$. Comparing the two control supplement groups, the ewes given LPS had significantly more SOD2 expression at hour 3 (CP $2.58 \pm 0.42$, CL $10.82 \pm 2.07, \mathrm{p}<0.0001)$ and hour 6 (CP $1.75 \pm 0.37$, CL $8.23 \pm 0.64, p=0.0017$ ) (Figure 18b).

\section{Reproductive System}

Progesterone

Neither supplement nor LPS treatment affected progesterone on day $9 / 10$ post coitus $(3.15 \pm 0.18$ $\mathrm{ng} / \mathrm{ml})$ or on day $25 / 26$ post coitus (3.90 $\pm 0.28 \mathrm{ng} / \mathrm{ml}$, Figure 19$)$.

\section{Pregnancy diagnosis/lambing data}

The number of ewes pregnant at 25/26 dpc in each group did not differ. Neither treatment nor feed affected loss of pregnancy. Logistic regression confirmed the contingency analyses: neither treatment, feed, nor the interaction could predict the odds of a ewe having a lamb $(\mathrm{p}=0.8156)$. The number of lambs born in each treatment group from mating that took place before the experiment did not differ among groups.

\section{Discussion}

In both trials, LPS caused fever, decreases in WBCs, muscosal responses, behavioral changes, increases in Hp, cortisol, and gene expression of CXCL8, TLR4, C3, SOD2, and decreases in gene expression of MRC1 and Foxp3.

Trial 1 showed beneficial effects of supplementary flaxseed on temperature response, change in WBCs, and APP and cortisol. APPs are non-specific markers of inflammation and used to restore homeostasis; an increase in these proteins in the FL compared to CL group could accelerate pathogen clearance and inflammation resolution in the flaxseed fed ewes. Plasma of cortisol were affected by supplement and LPS. The HPA axis is activated by $\mathrm{PGE}_{2}$ produced peripherally in response to LPS that 
circulates to the brain or produced by brain endothelial cells in response to PICs. Reduced cortisol levels in the flaxseed group suggests a dampened inflammatory response. LPS caused noticeable behavior and mucosal changes and there was no differences between ewes fed the different supplement $s$. These results agree with Farran et al. (2008) who fed supplement s with either ground flaxseed (12.9\% DM) or tallow (4\% DM) to beef steers for 14 days before LPS challenge $(0.2 \mu \mathrm{g} / \mathrm{kg}$ of BW). Lipopolysaccharide caused an increase in rectal temperature, $\mathrm{TNF} \alpha$, and $\mathrm{Hp}$, while decreasing WBCs within the first hour after challenge. Steers fed flaxseed had a reduced response in rectal temperature compared to the tallow fed steers.

The $\omega$-3 PUFAs in flaxseed can be incorporated into cellular membranes, which will influence cytokine production and the type of eicosanoids produced in response to inflammatory signals (Endres et al., 1989; Calder, 1996). Fever is induced by $\mathrm{PGE}_{2}$; decreased production of 2 series prostaglandins by switching AA for $\omega-3$ PUFAs in cell membranes may be the mechanism of flaxseed's effect on temperature response to LPS. ALA can be converted into EPA and DHA by animal cells (Calder, 1996), which can compete with AA for enzymes resulting in a reduction in production of PIMs such as the 2 series PGs and 4-series LTs (Calder, 1998). Indeed, when Farren et al. (2008) fed crossbred heifer calves a supplement containing ground flaxseed (12.9\% DM) for 35 days, plasma lipids were significantly enriched in ALA (C18:3n3) and EPA (C20:5n3) with a decrease in dihomo- $p$-linolenic acid (c20:3n6) compared to the heifers being fed supplement s with added tallow or rolled full-fat soybeans. The addition of fish oil (high omega 3 FAs) to supplement s of human volunteers resulted in reduced IL-1 $\beta$, IL- $1 \alpha$, and TNF $\alpha$ production from PBMCs stimulated in vitro with LPS, reduced $\mathrm{PGE}_{2}$ production when stimulated with $S$. epidermidis, and reduced neutrophil chemotaxis in response to $\mathrm{LTB}_{4}$ (measured as increase over random migration). The decreased production of PICs went along with a decreased ratio of AA to EPA in the membrane phospholipids of the volunteer's PBMCs (Endres et al., 1989).

With regard to gene expression of cytokines, chemokines, transcription factors, receptors, and enzymes involved in the inflammatory response, LPS increased expression of CXCL8, TLR4, C3, and SOD2, while decreasing expression of IFN $\gamma, \mathrm{MRC} 1$, and Foxp3. The flaxseed supplement decreased gene expression of CXCL8 and TLR 4. There was a flaxseed x LPS effect on gene expression of CXCL8, TLR4, and IL-6 with the increase in expression of the 3 genes in response to LPS being blunted by the flaxseed supplement indicating flaxseed dampens the inflammatory response. After controlling for the false discovery rate by the Benjamini-Hochberg procedure due to making multiple comparisons, the only effects that were still significant were the LPS effects. However, all the genes examined are involved in the inflammatory response, the sample size was small and due to pooling samples the time points had to serve as trials to analyze the data, which could have impaired the ability to find important differences 
between groups. Therefore, expression of all genes examined in trial 1 were examined in trial 2 with a larger sample size and time as an effect.

In trial 1, flaxseed fed ewes had greater plasma P4 on day 25/26 post coitus compared to control supplement fed ewes (Figure 12b). This could mean that flaxseed dampened inflammation induced changes in luteal function.

In trial 2, LPS caused an inflammation and APR evidenced by an increase in rectal temperature, $\mathrm{Hp}$, cortisol, a decrease in WBCs, and observable behavioral and mucosal changes. Neutrophils are the first responders to an inflammatory site. CXCL8 is one chemokine that recruits neutrophils; increased expression of this chemokine in LPS challenged ewes also demonstrates the occurrence of an inflammatory response (Figure 21a). LPS leads to the production of NO and ROS as these are used by immune cells to kill pathogens. These products can damage host cells and must be scavenged or neutralized. SOD2 is one enzyme responsible for protecting the host from damage; increased gene expression of SOD2 in the LPS challenged ewes indicates an inflammatory response (Figure 23b).

Lipopolysaccharide stimulates immune cells at the inflammatory site to produce PICs that can then circulate in the blood to the brain vasculature where they are either actively transported across the blood-brain barrier or penetrate into the brain through circumventricular organs. LPS also can stimulate cytokine production by endothelial cells in circumventricular organs (Breder et al., 1988; Spangelo et al., 1990; Netea et al., 2000). These pyrogenic cytokines (TNF $\alpha$, IL-6, IL-1 $\beta$ ) induce COX2 expression and $\mathrm{PGE}_{2}$ synthesis. Acting on the hypothalamus, $\mathrm{PGE}_{2}$ is the major mediator of the fever response (Engström et al., 2012; Wilhelms et al., 2014). These three PICs also mediate the effects of LPS on the HPA axis causing CRH release (McCann et al., 1995). They can activate the HPA axis independently or in combination resulting in synergistic effects (Sapolsky et al., 1987; Naitoh et al., 1988; Bernardini et al., 1990; Imura et al., 1991; Perstein et al., 1991; Perstein et al., 1993). During an infection or inflammatory response, TNF $\alpha$ is usually produced first, closely followed by IL-1 production and then IL-6 (Hesse et al., 1988; Akira et al., 1990; van Deventer et al., 1990). After cells have been initially stimulated to synthesize these cytokines, for example through PRR activation, these PICs can further stimulate their own production in an autocrine manner. In addition, TNF $\alpha$ and IL-1 stimulate secretion of each other, and both stimulate IL-6. However, IL-6 inhibits secretion of the other two cytokines and their effects on target tissues. GCs can inhibit the production of all 3 cytokines, but IL-6 is the least responsive to GC inhibition. Instead, IL-6 works with GCs to stimulate hepatic production of APPs (Hirano et al., 1990; Boumpas et al., 1993). Catecholamines stimulate IL-6 production, which will then inhibit TNF $\alpha$ and IL-1, stimulate GCs, and induce the APR (Chrousos, 1995). 
Lipopolysaccharide increased the average rectal temperature (from immediately before treatment until 6 hours post treatment) with the flaxseed supplement ing enhancing the febrile response (CP: 39.29 $\pm 0.07^{\circ} \mathrm{C}$; CL: $39.59 \pm 0.11^{\circ} \mathrm{C}$; FP: $38.94 \pm 0.04^{\circ} \mathrm{C}$; FL: $39.94 \pm 0.1^{\circ} \mathrm{C}$ ). An elevation in body temperature associated with infection could affect embryonic loss as heat stress in cattle that raised body temperature during embryonic development (Days 1 to 7 ) led to decreased survival of embryos recovered/assessed on day 7 (Putney et al., 1988). LPS increased temperature in both supplement groups but more so in the flaxseed-fed ewes, which may put their embryos at a greater risk of damage.

PICs along with stress hormones induce fever, sleepiness, fatigue, loss of appetite, decreased libido, and activate hepatic production of APPs; responses labeled as "sickness behavior" and the "acute phase response" (Elenkov and Chrousos, 1999). Observable discomfort was obvious in the LPS treated ewes noted as lethargy, lying down, inappetence, coughing, nasal discharge, and vaginal discharge but there was no effect of supplement .

WBCs dropped immediately after LPS treatment before gradually recovering back to normal levels by hour 6 . The drop in cells is likely due to extravasation into tissues. After returning to normal levels at hour 6, the number of cells continued to increase in the LPS groups likely a result of the inflammatory response stimulating hematopoiesis. Unlike in trial 1 where the flaxseed supplement hastened the recovery of normal cell counts, there was no difference in the response of WBCs to LPS between the two supplement treatments. Farran et al (2008) also reported no difference in WBC response after LPS challenge between supplement s containing ground flaxseed or tallow.

Similar to Farran et al. (2008), who did not observe a change in plasma Hp in LPS-challenged steers until 24 hours post treatment; ewes challenged with LPS had a delayed response in the APR. Plasma Hp in the FL ewes was not elevated until hour 12 and was still elevated at hour 24. Hp in plasma increased more gradually and less in the CL ewes, but the highest concentration still occurred at hour 24. In trial 1, the flaxseed supplement enhanced the increase in Hp levels in response to LPS, but there was no feed $x$ LPS effect in trial 2, likely due to the high variation in levels at hour 12. However, the FL ewes did have significantly more $\mathrm{Hp}$ at hour 24 compared to the CL ewes. SAA was affected only by time with the most fluctuation occurring in the FP group. Thus, it may not be a good marker of inflammation occurring in this LPS challenge model. Increased Hp in the FL ewes may accelerate pathogen clearance and inflammation resolution as $\mathrm{Hp}$ limits bacterial growth by binding free hemoglobin and reducing available iron (Cray et al., 2009), inhibiting prostaglandin synthase and $\mathrm{PGE}_{2}$ synthesis (Jue et al., 1983; Saeed et al., 1997) and can reduce cytokine production by PBMCs (Arredouani et al., 2005). APP production is stimulated by GCs and IL-6. There were no experimental effects on IL-6 gene expression, but the flaxseed supplement enhanced the LPS increase in cortisol, which could be associated with the 
increased Hp in this group. Cortisol increased immediately after LPS administration in the FL group and did not return to normal until hour 12 compared to the CL ewes that had a spike in cortisol only at hour 3 (Figure 20). Initial levels (hour 0) in the FL group were $54.06 \pm 9.85 \mathrm{ng} / \mathrm{ml}$ and peaked at hour 1 with $129.67 \pm 11.3 \mathrm{ng} / \mathrm{ml}$ (difference $75.55 \pm 1.46 \mathrm{ng} / \mathrm{ml}$ ) before slowly declining. Initial levels (hour 0) in the CL group were $28.73 \pm 5.72 \mathrm{ng} / \mathrm{ml}$ with spike to $81.59 \pm 61.55 \mathrm{ng} / \mathrm{ml}$ (difference $52.87 \pm 34.57 \mathrm{ng} / \mathrm{ml}$ ).

Activation of the HPA axis has both anti- and pro-inflammatory effects. The end products of the adaptive response to stress, cortisol from the adrenal gland and catecholamines (epinephrine $\&$ norepinephrine) from the sympathetic nervous system, have anti-inflammatory effects (Chrousos, 1995; Elenkov and Chrousos, 1999; Agelaki et al., 2002). Glucocorticoids can prevent leukocyte migration from the circulation into extravascular fluid spaces, reduce monocyte and granulocyte accumulation at the inflammation site, and suppress the production and/or activation of certain cytokines and inflammatory mediators (Chrousos, 1995).

Effects of GC on leukocyte and granulocyte migration are due to inhibition of adhesion molecules and their corresponding receptors (Cronstein et al., 1992). Glucocorticoids control inflammation by suppressing transcription of genes for the enzymes PLA2, COX2, and NOS responsible for producing prostanoids, platelet activating factor, and nitric oxide (Nakano et al., 1990; O’Banion et al., 1992; Boumpas et al., 1993; Moncada and Higgs, 1993). Catecholamines, acting on adrenergic receptors expressed by immune cells, stimulate intracellular cAMP production that suppresses chemotaxis, the release of inflammatory mediators, the lytic activity of NK cells and cytotoxic T cells, and the proliferation of T cells (Madden et al., 1995).

Stress hormones favor a humoral response rather than a cell-mediated response through control of cytokine production by inhibition of transcription factors like AP-1 and NF-kB (Elenkov et al., 1999). Both GCs (Ramírez et al., 1996) and catecholamines (Madden et al., 1995) inhibit IL-2 and IFN $\gamma$ production by Th1 cells (Elenkov et al., 1999). Furthermore, dexamethasone, epinephrine and norepinephrine inhibited the LPS-induced IL-12 production by human peripheral blood mononuclear leukocytes in vitro (Elenkov et al., 1996). Glucocorticoids not only inhibit IL-12 production by APCs but also downregulate IL-12 receptors on T and NK cells, favoring Th2 polarization since IL-12 is a major cytokine for polarization of Th1 cells (Elenkov and Chrousos, 1999). Shifting from Th1 to Th2 polarization will decrease IFN $\gamma$ production, as this cytokine is produced by Th1 cells. In addition, catecholamines (norepinephrine and epinephrine) stimulate IL-10 production, which stimulates Th2 polarization (Elenkov et al., 1996). Due to these various anti-inflammatory and immune modulating effects of GCs, the greater cortisol in the FL ewes may accelerate resolution of inflammation and limit inflammation at the maternal-fetal interface, which is critical for preventing direct damage to the embryo as well as maintaining luteal function and maternal-fetal tolerance. 
Lipopolysaccharide challenge caused gene expression of TLR4 to increase until hour 3 before returning to normal by hour 9. The FL group peaked at a higher fold change compared to the CL group but the change from hour 0 to 3 between the two LPS groups was similar and there was no Feed or Feed $\mathrm{x}$ LPS effect. Although there are no treatment, supplement, or interaction effect on fold change in gene expression of the transcription factor NF-kB, the PICs TNF $\alpha$ and IFN $\gamma$, the enzyme COX2, or the antiinflammatory cytokines IL-10 or TGF $\beta$, gene expression does not always reflect protein levels or provide information about activity. TLR4 is known to lead to activation of the transcription factors NF-kB and AP-1 to promote inflammation. The febrile response, HPA axis activation, and APP production are all downstream of PICs and $\mathrm{PGE}_{2}$ suggesting NF-kB and COX2 are active in the LPS treated ewes and TNF $\alpha$, IL-1 $\beta$, IL-6, and $\mathrm{PGE}_{2}$ are being produced, even though that is not reflected in the gene expression data. In addition, Farran et al (2008) reported elevated TNF- $\alpha$ protein in LPS challenged steers, and LPS has been shown to increase TNF $\alpha$ plasma levels in sheep (Graham, 2014). The flaxseed fed ewes had an increased febrile response, cortisol production, and Hp production, and tended to have a greater increase in TLR4 expression when treated with LPS compared to CL ewes. Downstream of TLR4 activation, the FL ewes could have greater production of PICs, enhanced activity of COX2 and $\mathrm{PGE}_{2}$ production but this cannot be determined from the present results.

Peroxisome proliferator-activated receptors are transcription factors with anti-inflammatory functions (Clark et al., 2000; Dinarello, 2010; Li et al., 2014; Lian et al., 2015). After binding to an agonist, PPARs reduce expression of PICs, chemokines, and adhesion molecules by interfering with other transcription factors (Ricote et al., 1998; Clark et al., 2000; Lappas et al., 2002; Genolet et al., 2004; Feige et al., 2006; Szanto and Nagy, 2008; Dinarello, 2010; Li et al., 2014; Lian et al., 2015). The 15LOX metabolites of ALA, 13-(S)-hydroperoxyoctadecatrienoic acid (13-(S)-HPOTrE) and 13-(S)hydroxyoctadecatrienoic acid (13-(S)-HOTrE), acting on PPAR $\gamma$ had anti-inflammatory effects in LPS stimulated RAW 264.7 cells (MФ cell line) and in mouse peritoneal MФs. The anti-inflammatory effects seen in cultured MФs included decreasing levels of LPS-induced NO and ROS, increasing expression of IL-10, decreasing expression of iNOS, IL-1 $\beta$, IL-18, NLRP3 and TNF $\alpha$, and decreasing NF- $\mathrm{B}$ translocation. Inhibition of NLRP3 inflammasome activation by the metabolites also contributes to reduced IL-1 $\beta$ production in response to LPS. In vivo, mice pre-treated with the metabolites had a higher survival rate and displayed less sickness behaviors after LPS injection, while mice that received the metabolites and underwent cecal ligation and puncture (sepsis induced by polymicrobial infection) had decreased expression of NLRP3, iNOS and IL-1 $\beta$ with an increase in IL-10 compared to controls (Kumar et al., 2016). PPAR $\gamma$ expression decreased in response to LPS challenge in ewes and there was no supplement effect (Figure 24a). Flaxseed is rich in ALA, therefore, the flaxseed ewes potentially had 
more PPAR $\gamma$ agonists that could have had anti-inflammatory effects. However, flaxseed-fed ewes did not have increased IL-10 expression, decreased TNF $\alpha$ expression, or display less sickness symptoms when compared to control supplement fed ewes when challenged with LPS.

RAW 264.7 cells pretreated with ALA metabolites before LPS stimulation had increased COX2 expression and levels of $\mathrm{PGE}_{2}$ and $\mathrm{PGD}_{2}$ (Kumar et al., 2016). $\mathrm{PGD}_{2}$ can be converted into $\mathrm{PGJ}_{2}$ and the metabolites ${ }^{12} \Delta \mathrm{PGJ}_{2}$ and $15 \mathrm{~d}-\mathrm{PGJ}_{2}$ (a natural ligand of PPAR $\gamma$ ) (Scher and Pillinger, 2005). Activation of PPAR $\gamma$ and increased PGE $_{2}$ (Heusinkveld et al., 2011; Li et al., 2014) has been associated with a change in MФ subtype from M1 to M2 cells (Castrillo and Tontonoz, 2004). Kumar et al. (2016) reported activation of PPAR $\gamma$ and increased $\mathrm{PGE}_{2}$ levels in MФs exposed to ALA metabolites. A switch in MФ subtype may be involved in the anti-inflammatory effects of ALA metabolites. It is important that the majority of MФs at the maternal-fetal interface are the M2 phenotype to maintain fetal tolerance; feeding flaxseed could help maintain the tolerogenic phenotypes of immune cells at the maternal-fetal interface through ALA metabolites interacting with PPARY.

Prostaglandins such as $\mathrm{PGE}_{2}$ are produced during the initial inflammation phase (Levy et al., 2001) that are involved in the early steps in the control of blood flow and vessel dilation needed for leukocytes to undergo firm adhesion and diapedesis (Williams and Peck, 1977). In addition to chemokines, $\mathrm{LTB}_{4}$ controls trafficking of neutrophils from the post capillary lumen to the interstitial space (Pouliot et al., 2002). Production of $\mathrm{PGE}_{2}$ occurs early in infection/inflammation through upregulation of COX2 and PGE synthase 1 in MФs, endothelial cells, and DCs. Prostaglandin $\mathrm{E}_{2}$ has pro- and antiinflammatory effects (Fullerton et al., 2014). Binding to prostaglandin E receptors (EP1, EP2, EP3, EP4), PGE2 is responsible for several of the cardinal signs of inflammation early in a response such as pain, edema, local heat production, fever, and vasodilation. $\mathrm{PGE}_{2}$ also enhances $\mathrm{LTB}_{4}$ mediated neutrophil extravasation and increases vascular permeability (Bray et al., 1981; Serhan 2007; Lazarus et al., 2007; Serhan 2014). Later in the response, $\mathrm{PGE}_{2}$ causes expression of 12/15-LOX in cells such as MФs and neutrophils leading to production of SPMs that resolve the inflammation. Therefore, $\mathrm{PGE}_{2}$ plays a controlling role in eicosanoid class switching (switch from pro-inflammatory PG production to antiinflammatory lipoxins production) (Levy et al., 2001; Serhan and Savill, 2005; Norris et al., 2014). In addition, inactivation of $\mathrm{PGE}_{2}$ by prostaglandin dehydrogenase converts $\mathrm{PGE}_{2}$ into 15-keto- $\mathrm{PGE}_{2}$ which is a ligand for PPARy, a receptor with anti-inflammatory effects (Harmon et al., 2010; Dennis and Norris, 2015).

Macrophages have been shown to have the EP2 and EP4 receptors and respond to $\mathrm{PGE}_{2}$ by decreasing TNF $\alpha$ production and increasing IL-10 production, a result of regulation of cytokine gene expression. The increased IL-10 may be involved in decreasing TNF $\alpha$ production (Kunkel et al., 1988; 
Renz et al., 1988; Shinomiya et al., 2001; Treffkorn et al., 2004). Paracrine or autocrine $\mathrm{PGE}_{2}$ signaling at the inflammatory site may play a role in switching production of cytokines from pro- to antiinflammatory (Shinomiya et al., 2001). Cyclooxygenase 2 expression in the 4 experimental groups fluctuated over time without any effect of supplement or treatment and which eicosanoids were produced in each group of ewes is not known. However, the enhanced febrile response in the FL ewes is indicative of more $\mathrm{PGE}_{2}$ production. The combination of more $\mathrm{PGE}_{2}$ that is involved in initiation of inflammation resolution and is responsible for eicosanoid class switching with the flaxseed providing $\omega-3$ PUFAs (substrates to make SPMs) potentially encourages a more rapid resolution of inflammation, which would be critical at the maternal-fetal interface.

Lipopolysaccharide challenge increased gene expression of C3 and decreased expression of MRC1 and FOXP3 but there was no difference in response to LPS between the two supplements. The role of complement is to kill pathogens enough to keep the infection from spreading while an adaptive immune response is being generated, to promote inflammation by causing production of PICs, to recruit additional immune cells and to opsonize pathogens and immune complexes to target them for clearance by phagocytes (Molina, 2005; Murphy, 2012). Complement activation during pregnancy has been shown to promote inflammation and embryo loss (Xu et al., 2000; Mellor et al., 2001; Mao et al., 2003; Caucheteux et al., 2003). Increased C3 expression in the LPS-treated ewes indicates gram negative bacterial infections can cause embryonic loss through complement activation.

Mannose receptor C 1 binds to and is involved in endocytosis of glycoproteins. It is expressed on MФs and DCs to help with neutralization and clearance of pathogens (Shepard et al., 1990; Engering et al., 1997). A decrease in MRC1 expression during a bacterial infection could hinder pathogen clearance, delay inflammation resolution and increase the chance of embryonic loss.

Forkhead box P3 is the transcription factor that regulates pathways that give Treg cells their immune suppressive functions. Tregs cells are critical for controlling inflammation at the maternal-fetal interface and generating maternal-fetal tolerance (Fiorentino et al., 1991b; Fallarino et al., 2003; Ghiringhelli et al., 2005a; Mahnke et al., 2007; Shevach et al., 2009). Reduced numbers of Tregs or diminished suppressive function of these cells result in embryonic loss (Aluvihare et al., 2004; Zenclussen et al., 2005; Zhao et al., 2007). Tregs are not a terminally differentiated cell type; in the presence of high amounts of PICs they can turn into effector T cells (Gao et al., 2012). Reduced Foxp3 expression in ewes challenged with LPS could indicate a reduction in Treg cells, perhaps because they are switching into inflammatory $\mathrm{T}$ cell subsets, which would be detrimental to pregnancy success. Expression of Foxp3 in the LPS-challenged ewes does start to rebound back to normal, which could be due to the short-lived response (hours) to the LPS challenge. An actual bacterial infection would take a lot longer to 
clear and the prolonged inflammatory response is likely to have negative consequences on Treg cell numbers and function resulting in inflammation induced damage to the embryo, loss of luteal function, and loss of maternal-fetal tolerance.

Ovarian P4, conceptus IFN- $\tau$, and PGs regulate endometrial functions important for conceptus elongation and implantation during the peri-implantation period of pregnancy (Dorniak et al., 2011). P4 action for 8-10 days induces expression of elongation and implantation related genes in the endometrial luminal epithelium and glandular epithelium (Simmons et al., 2010; Charpigny et al., 1997a; Eggleston et al., 1990). Some of the gene changes are mediated by cortisol and PGs. P4 upregulates HSDI1B1 and PTGS2 expression while IFN- $\tau$ affects their activity (Simmons et al., 2010; Dorniak et al, 2013b). Cortisol also induces $H S D 11 B 1$ expression needed for production of cortisol from corticosterone (Simmons et al., 2010). Infusing cortisol into the uterus of cyclic ewes from day 10-14 after estrus, Dorniak et al. (2013b) showed that cortisol stimulates endometrial HSD11B1 expression and activity, increases endometrial PTGS2 activity and the amount of PG in the uterine lumen, and up-regulates many conceptus elongation-related genes in the endometrium. $\mathrm{PGE}_{2}$ and $\mathrm{PGI}_{2}$, produced by prostaglandin synthase 2, mediate some of P4's effects on gene expression as well as regulating blastocyst implantation, decidualization, and uterine angiogenesis (Dorniak et al., 2011). Inflammation/infections can 1: lead to luteolysis and reduced P4 levels, 2: trigger production of PICs that cause apoptosis of trophoblast cells

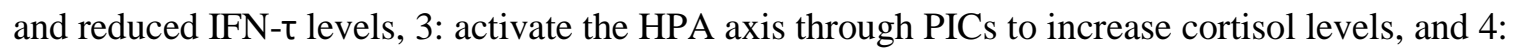
stimulate PG production. These changes in mediators that regulate elongation- and implantation-related genes could result in asynchrony of an implantation-competent embryo and a receptive uterus, thus the "window of implantation" being missed, resulting in implantation failure. Lipopolysaccharide challenge on day 5/6 post coitus caused activation of the HPA axis and cortisol production, which may alter timing of the expression of uterine genes. The short-lived inflammatory response to the one LPS dose in the experiment may not cause much damage in regards to cortisol altering gene expression in the uterus, but a prolonged bacterial infection could affect pregnancy success in this manner.

There was no difference in P4 levels on either 8/9 or 25/26 dpc indicating LPS treatment did not affect luteal function. Neither pregnancy diagnosis by ultrasound nor lambing data revealed differences between the treatment groups in regards to pregnancy success suggesting the LPS challenge did not cause embryonic loss and the flaxseed supplement did not improve pregnancy rates. However, the sample size of this study was small compared to other studies that have used hundreds of ewes to examine embryonic loss (Dixon et al., 2014).

In both $\omega$-3 PUFA studies, feed analysis of samples of the prepared supplement s reported more neutral detergent fiber (NDF) and less non-fiber carbohydrate (NFC) in the flaxseed supplement. NDF is 
a reflection of the amount of structural plant components. Therefore, it makes sense that the flaxseed, with a seed coat, had higher NDF than the control supplement with added cracked corn, soybean meal, and soybean oil (SBO). NFC consists of starch, simple sugars, and soluble fiber and the calculation to determine NFC includes percent NDF; therefore, it makes sense that the flaxseed supplement that had a higher percent NDF, had a lower NFC percent. It also fits that the control supplement had a higher percent NFC because the components of the supplement (cracked corn) would have provided additional starch. Supplement s with low NDF/high NFC, like the control supplement compared to the flaxseed supplement, can cause ruminal acidosis and decrease the rate of VFA absorption from the rumen. However, the ewes in the present study were on pasture, and grass would have increased fiber intake. In addition, the amount being fed was split between two feedings per day, and the rumen was not exposed to all of the starch at once. Both supplement s were eaten readily when presented and there were no changes in body weight of the ewes.

The addition of unprotected fats to ruminant supplement $\mathrm{s}$ can decrease dry matter intake and reduce digestibility of non-lipid energy sources as a result of changes to ruminal fermentation, gut motility, palatability, release of gut hormones, and oxidation of fat in the liver (Allen, 2000). Numerous studies feeding flaxseed to ruminants in various forms (whole, ground, rolled, extruded) have investigated effects on digestibilities, rumen health, VFA production, and incorporation of fats into tissues and milk. Overall, feeding flaxseed did not have negative effects on ruminant digestion, and $\omega$-3 PUFAs were available for use by the animal to incorporate into tissues and milk. This was especially true when feeding whole flaxseed since the seed coat helped to protect the oil from rumen bacteria (Wachira et al., 2000; Petit et al., 2002; Gonthier et al., 2004; Scholljegerdes and Kronberg, 2008; Petit and Côrtes et al., 2010; Côrtes et al., 2010; Mesgaran et al., 2012; Schroeder et al., 2014). Even though flaxseed has a hard outer coat, ruminants are able to extract and utilize fats from the seeds post-rumen (Oba et al., 2009).

Soybean oil was one of the components used in the control supplement of the present experiments to match the flaxseed in fat and crude protein content using the Pearson Square method. Soybean oil contains unprotected fat and thus can potentially be more damaging to rumen health than the flaxseed. However, Wonsil et al. (1994) fed Holstein cows an amount of SBO per pound of animal per day comparable to that fed in the present studies (Wonsil et al.: $0.0012 \mathrm{lb} \mathrm{SBO} / \mathrm{lb}$ cow/day, present study: $0.0011 \mathrm{lb} \mathrm{SBO} / \mathrm{lb}$ ewe/day). Compared to their control supplement that contained no supplemental fat, the addition of SBO did not affect total or individual VFA concentrations or the ratio of acetate to propionate. There were no changes in dry matter intake or apparent digestibility (in the rumen or total tract) of dry matter, acid detergent fiber, organic matter or nitrogen, indicating that SBO was not toxic to rumen microorganisms (Wonsil et al., 1994). There is no information on rumen health or digestibilities of the supplement s for the present studies; however, based on previous studies that supplemented comparable 
amounts of flaxseed or SBO and the fact that weights of the ewes (recorded on: the day the supplement started, after 7 days on the supplement, and the day the supplement was stopped: 28 days) did not change (data not shown) it should be fair to say the experimental supplement did not negatively affect digestion in ewes.

Fat in SBO is $14 \%$ saturated (10\% palmitic; $4 \%$ stearic) and $81 \%$ unsaturated (51\% linoleic; $23 \%$ oleic; 7\% linolenic) (Gunstone, 1996) with most of the fat being linoleic. While SBO contains some ALA, levels are way below the ALA content in flaxseed (53\% of the total FAs). In addition, the fat in $\mathrm{SBO}$ is not protected from rumen microbes and will be subject to biohydrogenation increasing duodenal flow of stearic and oleic acid, not $\omega-3$ PUFAs. Therefore, even though SBO contains some ALA, feeding SBO does not change circulating levels of ALA, EPA, or DHA (Wonsil et al., 1994). The high ALA content in whole flaxseed is protected from rumen biohydrogenation by the seed coat, however, making $\omega$-3 PUFAs available to the animal (Oba et al., 2009).

Omega-3 PUFAs are known to have anti-inflammatory effects, and it was hypothesized that feeding flaxseed to ewes would dampen the inflammatory response to an LPS challenge. Between the two trials, the effect of flaxseed when LPS was given was not consistent for all measurements. In trial 1 , flaxseed dampened both the febrile response and HPA axis activation in response to LPS but enhanced the temperature increase and cortisol levels in trial 2. TLR4 gene expression in response to LPS was dampened with flaxseed in trial 1, but tended to be increased in trial 2 compared to the CL group, but did not reach statistical significance. In trial 1, the flaxseed supplement hastened the recovery of the decrease in WBC counts in reponse to LPS but there was no difference in the second study. Flaxseed enhanced the LPS increase in Hp in trial 1 and tended to do so in trial 2. Possible explanations for the discrepancies include small sample sizes (especially in trial 1), the amount of flaxseed supplemented might have been insufficient to get significant anti-inflammatory effects, the feeding period may have been inadequate $(\sim 8$ days) to get a significant amount of $\omega$-3 PUFAs incorporated into cellular membranes before LPS was administered, and the fat content of the flaxseed was greater in trial $1(39.11 \%)$ compared to trial 2 (32.89\%), which would have provided less $\omega-3$ PUFAs to the ewes in trial 2. Another likely possibility is that the ewes in the spring had a higher intake of $\omega-3$ PUFAs due to spring grass being higher in $\omega-3$ PUFA than fall grass, and both sets of ewes were kept on pasture. In conclusion, additional studies need to be conducted that address the above mentioned issues before stating that flaxseed has beneficial effects with regards to inflammation induced by LPS treatment. 


\section{Tables}

Table 1: Analysis of whole flaxseed used in trial 1 of the $\omega$-3 PUFA study (Spring 2015). Whole flaxseed was purchased from Valley Point Feed (Albright, WV; Lot \# DM1415) and analyzed by the Rumen Fermentation Profiling Laboratory at WVU.

\begin{tabular}{|l|c|c|}
\hline & \%"As Sampled" & \%Dry Matter Basis \\
\hline \%Dry Matter* & 91.38 & \\
\hline \% Moisture & 8.62 & \\
\hline \% Crude Protein & 15.84 & 17.33 \\
\hline \% Crude Fat & 39.11 & 42.80 \\
\hline$\% \mathrm{TDN}$ & 118.70 & 129.9 \\
\hline $\mathrm{NE}_{\mathrm{G}}, \mathrm{Mcal} / \mathrm{lb}$ & 1.14 & 1.25 \\
\hline $\mathrm{NE}_{\mathrm{M}}, \mathrm{Mcal} / \mathrm{lb}$ & 1.44 & 1.58 \\
\hline $\mathrm{NE}_{\mathrm{L}}, \mathrm{Mcal} / \mathrm{lb}$ & 1.50 & 1.64 \\
\hline $\begin{array}{l}\text { *Dry Matter of sample as } \\
\text { received. }\end{array}$ & & \\
\hline
\end{tabular}

Table 2: Composition of supplements fed to ewes in trial 1 of the $\omega-3$ PUFA study. Ewes received a total of $1 \mathrm{lb} /$ per day. *Sheep ration consisted of cracked corn, soybean meal, minerals, limestone, and Bovatec 58 and was already prepared by WVU farm crew. Based on flaxseed analysis (Table 1), cracked corn, soybean meal, and soybean oil were used to match the flaxseed in fat content $(39.11 \%)$ and crude protein $(15.84 \%)$.

\begin{tabular}{|l|l|l|l|}
\hline Ingredient & & $\begin{array}{l}\text { Flaxseed } \\
\text { Supplement }\end{array}$ & $\begin{array}{l}\text { Control } \\
\text { Supplement }\end{array}$ \\
\hline $\begin{array}{l}\text { Sheep } \\
\text { Ration* } \\
\text { (total .45lb) }\end{array}$ & $\begin{array}{l}\text { Cracked Corn } \\
\text { (76.69\%) }\end{array}$ & $0.35 \mathrm{lb}$ & $\begin{array}{l}0.35 \mathrm{lb} \\
+ \\
0.2 \mathrm{lb} \\
(36.36 \%)\end{array}$ \\
\hline & $\begin{array}{l}\text { Soybean Meal } \\
(11.89 \%)\end{array}$ & $0.054 \mathrm{lb}$ & $\begin{array}{l}0.054 \mathrm{lb} \\
+ \\
0.21 \mathrm{lb} \\
(38.18 \%)\end{array}$ \\
\hline & $\begin{array}{l}\text { Sheperd's pride } \\
\text { minerals }(8.86 \%)\end{array}$ & $0.04 \mathrm{lb}$ & $0.04 \mathrm{lb}$ \\
\hline & Limestone (2.53\%) & $0.011 \mathrm{lb}$ & $0.011 \mathrm{lb}$ \\
\hline Bovatec 68 & $0.0023 \mathrm{lb}$ & $0.00231 \mathrm{~b}$ \\
(0.05\%) & & 0 \\
\hline $\begin{array}{l}\text { Whole } \\
\text { Flaxseed }\end{array}$ & & $55 \mathrm{lb}$ & $0.14 \mathrm{lb}$ \\
\hline Soybean Oil & & 0 & \\
\hline
\end{tabular}


Table 3: Results from feed analysis conducted on samples of the prepared supplements used in trial 1 of the $\omega$-3 PUFA study. Samples were analyzed by the Rumen Fermentation Profiling Laboratory at WVU. T-tests were used to compare each variable between supplements; Bonferroni correction (0.05/10) was used to control for multiple comparisons. $\alpha=0.005$ Results reported as mean \pm SEM.

\begin{tabular}{|l|l|l|l|}
\hline Variable & $\begin{array}{l}\text { Control Supplement } \\
(\mathbf{n = 4})(\mathbf{m e a n} \pm \text { SEM })\end{array}$ & $\begin{array}{l}\text { Flaxseed Supplement } \\
(\mathbf{n = 4})(\mathbf{m e a n} \pm \text { SEM })\end{array}$ & P value \\
\hline Percent Dry Matter & $90.55 \pm 0.104$ & $90.15 \pm 0.24$ & 0.1931 \\
\hline Percent Moisture & $9.45 \pm 0.104$ & $9.86 \pm 0.24$ & 0.1931 \\
\hline Percent Crude Protein & $15.49 \pm 0.585$ & $16.58 \pm 1.071$ & 0.4144 \\
\hline Percent Crude Fat & $21.52 \pm 1.383$ & $16.57 \pm 1.09$ & 0.0326 \\
\hline $\begin{array}{l}\text { Percent Neutral Detergent } \\
\text { Fiber }\end{array}$ & $6.77 \pm 0.281$ & $19.70 \pm 0.776$ & $\mathbf{0 . 0 0 0 1}$ \\
\hline Percent Ash & $5.20 \pm 0.371$ & $3.90 \pm 0.392$ & 0.053 \\
\hline $\begin{array}{l}\text { Percent Non-Fiber } \\
\text { Carbohydrate }\end{array}$ & $41.58 \pm 0.907$ & $33.39 \pm 0.666$ & $\mathbf{0 . 0 0 0 5}$ \\
\hline $\begin{array}{l}\text { Percent Total Digestible } \\
\text { Nutrients }\end{array}$ & $76.26 \pm 0.922$ & $74.00 \pm 0.40$ & 0.084 \\
\hline Net Energy for Gain & $0.88 \pm 0.013$ & $0.85 \pm 0.005$ & 0.0938 \\
\hline Net Energy for Maintenance & $0.59 \pm 0.012$ & $0.56 \pm 0.007$ & 0.1078 \\
\hline
\end{tabular}


Table 4: Primer sequences of the housekeeping and genes of interest.

\begin{tabular}{|c|c|c|c|c|}
\hline Gene Name & $\begin{array}{l}\text { Gene } \\
\text { Symbol }\end{array}$ & $\begin{array}{l}\text { Forward Primer (5'-3') } \\
\text { Reverse Primer (5'-3') }\end{array}$ & $\mathrm{bp}$ & $\mathrm{T}_{\mathrm{M}}{ }^{\mathrm{O}} \mathrm{C}$ \\
\hline $\begin{array}{l}\text { Complement component } \\
3\end{array}$ & $\mathrm{C} 3$ & $\begin{array}{l}\text { GCACTGTCCACCAACCTCA } \\
\text { ATCAGGCTTCTGCTTCTCCA }\end{array}$ & 87 & 81.5 \\
\hline Cyclooxygenase 2 & $\mathrm{COX} 2$ & $\begin{array}{l}\text { CTACCCGCCTCATATTCCTG } \\
\text { CCAAATGGTGGCATACATCA }\end{array}$ & 94 & 82 \\
\hline Forkhead box P3 & Foxp3 & $\begin{array}{l}\text { GAAACAGCACATTCCCAGAGT } \\
\text { GGATGAGGGTGGCATAGGT }\end{array}$ & 90 & 80.5 \\
\hline $\begin{array}{l}\text { Glyceraldehyde 3- } \\
\text { Phosphate } \\
\text { Dehydrogenase }\end{array}$ & GAPDH & $\begin{array}{l}\text { CAGGAGCACGAGAGGAAGAG } \\
\text { AATGTATGGAGGTCGGGAGA }\end{array}$ & 91 & 83 \\
\hline Interferon gamma & $\overline{\mathrm{IFN} \gamma}$ & $\begin{array}{l}\text { ATGACCTGTCGCCAAAATC } \\
\text { GCAGGCAGGAGAACCATTAC }\end{array}$ & 97 & 81 \\
\hline Interleukin 6 & IL-6 & $\begin{array}{l}\text { TAACCACTCCAGCCACACAC } \\
\text { GATAACCTTTGCGTTCTTTACCC }\end{array}$ & 80 & 79.5 \\
\hline Interleukin 8 & CXCL8 & $\begin{array}{l}\text { GGATTCACGAGTTCCTGTTCA } \\
\text { CTGTGAGGTAGAAAGATGACTGAGA }\end{array}$ & 86 & 77 \\
\hline Interleukin 10 & IL-10 & $\begin{array}{l}\text { TTTCCCTGACTGCCCTCTAA } \\
\text { GCTCCCTGGTTTCTCTTCCT }\end{array}$ & 105 & 82 \\
\hline $\begin{array}{l}\text { Mannose Receptor, type } \\
\text { C } 1\end{array}$ & MRC1 & $\begin{array}{l}\text { GGAGGGAACACAAGGGATG } \\
\text { GCAAGGAAGGGTCAGGTTG }\end{array}$ & 94 & 78.5 \\
\hline Nuclear Factor kappa B & $\mathrm{NF}-\kappa \mathrm{B}$ & $\begin{array}{l}\text { ATTCAGCCCTTTGCCCATCT } \\
\text { ATGGGATGTCAGTGGCGTTA }\end{array}$ & & \\
\hline $\begin{array}{l}\text { Peroxisome Proliferator } \\
\text { Activated Receptor } \\
\text { gamma }\end{array}$ & $\operatorname{PPAR} \gamma$ & $\begin{array}{l}\text { CTTGACGGGAAAGACGACA } \\
\text { GCTGATGTGCTTGAACTTGATT }\end{array}$ & 97 & 75.5 \\
\hline Superoxide Dismutase 2 & SOD2 & $\begin{array}{l}\text { TCACAGCATCTTCTGGACAA } \\
\text { TGCTCCTTATTGAAGCCAAG }\end{array}$ & & \\
\hline Toll Like Receptor 4 & TLR4 & $\begin{array}{l}\text { GGCATCATCTTCATCGTCCT } \\
\text { CCACTCCAGGTAGGTGTTCC }\end{array}$ & 99 & 82.5 \\
\hline
\end{tabular}

Table 5: Analysis of the whole flaxseed used in trial 2 of the $\omega$-3 PUFA study. Whole flaxseed was purchased from Valley Point Feed (Albright, WV; Lot \# 15-0072 GS1 Lot: (10)1-22373) and analyzed by the Rumen Fermentation Profiling Laboratory at WVU.

\begin{tabular}{|l|c|c|}
\hline & \%"As Sampled" & \%Dry Matter Basis \\
\hline \%Dry Matter* & 92.77 & \\
\hline \% Moisture & 7.23 & \\
\hline \% Crude Protein & 21.01 & 22.65 \\
\hline \% Crude Fat & 32.89 & 35.45 \\
\hline \%DN & 115.2 & 124.2 \\
\hline $\mathrm{NE}_{\mathrm{G}}, \mathrm{Mcal} / \mathrm{lb}$ & 1.09 & 1.18 \\
\hline $\mathrm{NE}_{\mathrm{M}}, \mathrm{Mcal} / \mathrm{lb}$ & 1.39 & 1.50 \\
\hline $\mathrm{NE}_{\mathrm{L}}, \mathrm{Mcal} / \mathrm{lb}$ & 1.41 & 1.52 \\
\hline $\begin{array}{l}\text { *Dry Matter of } \\
\text { sample as received. }\end{array}$ & & \\
\hline
\end{tabular}


Table 6: Composition of supplements fed to ewes in trial 2 of the $\omega-3$ PUFA study. Ewes received a total of $1 \mathrm{lb} /$ per day. *Sheep ration consisted of cracked corn, soybean meal, minerals, limestone, and Bovatec 58 and was already prepared by WVU farm crew. Based on flaxseed analysis (Table 5), cracked corn, soybean meal, and soybean oil was used to match the flaxseed in fat content (32.89\%) and crude protein $(21.01 \%)$.

\begin{tabular}{|l|l|l|l|}
\hline Ingredient & & $\begin{array}{l}\text { Flaxseed } \\
\text { Supplement }\end{array}$ & $\begin{array}{l}\text { Control } \\
\text { Supplement }\end{array}$ \\
\hline $\begin{array}{l}\text { Sheep } \\
\text { Ration* } \\
\text { (total .45lb) }\end{array}$ & $\begin{array}{l}\text { Cracked Corn } \\
\text { (76.69\%) }\end{array}$ & $0.35 \mathrm{lb}$ & $\begin{array}{l}0.35 \mathrm{lb} \\
+ \\
0.1694 \mathrm{lb} \\
(30.81 \%)\end{array}$ \\
\hline & $\begin{array}{l}\text { Soybean Meal } \\
(11.89 \%)\end{array}$ & $0.054 \mathrm{lb}$ & $\begin{array}{l}0.054 \mathrm{lb} \\
+ \\
0.2089 \mathrm{lb} \\
(37.98 \%)\end{array}$ \\
\hline & $\begin{array}{l}\text { Sheperd's pride } \\
\text { minerals }(8.86 \%)\end{array}$ & $0.04 \mathrm{lb}$ & $0.04 \mathrm{lb}$ \\
\hline & $\begin{array}{l}\text { Limestone (2.53\%) } \\
\text { Bovatec 68 } \\
\text { (0.05\%) }\end{array}$ & $0.011 \mathrm{lb}$ & $0.011 \mathrm{lb}$ \\
\hline & & $0.00231 \mathrm{~b}$ & $0.00231 \mathrm{~b}$ \\
\hline $\begin{array}{l}\text { Whole } \\
\text { Flaxseed }\end{array}$ & & $.55 \mathrm{lb}$ & 0 \\
\hline Soybean Oil & & 0 & $\begin{array}{l}0.1716 \mathrm{lb} \\
(31.20 \%)\end{array}$ \\
\hline
\end{tabular}


Table 7: Results from feed analysis conducted on samples of the prepared supplements used in trial 2 of the $\omega$-3 PUFA study. Samples were analyzed by the Rumen Fermentation Profiling Laboratory at WVU. T-tests were used to compare each variable between supplements; Bonferroni correction used to control for multiple comparisons. $\alpha=0.005$ Results reported as mean \pm SEM.

\begin{tabular}{|l|l|l|l|}
\hline Variable & $\begin{array}{l}\text { Control Supplement }(\mathbf{n}=7) \\
(\mathbf{m e a n} \pm \mathbf{S E M})\end{array}$ & $\begin{array}{l}\text { Flaxseed Supplement }(\mathbf{n}=5) \\
(\mathbf{m e a n} \pm \mathbf{S E M})\end{array}$ & P value \\
\hline Percent Dry Matter & $89.01 \pm 0.213$ & $89.84 \pm 0.118$ & 0.0128 \\
\hline Percent Moisture & $10.99 \pm 0.213$ & $10.16 \pm 0.118$ & 0.0128 \\
\hline Percent Crude Protein & $18.18 \pm 0.625$ & $16.81 \pm 0.417$ & 0.1283 \\
\hline Percent Crude Fat & $13.63 \pm 0.364$ & $13.76 \pm 2.236$ & 0.8959 \\
\hline Percent Neutral Detergent Fiber & $10.48 \pm 0.863$ & $21.39 \pm 1.852$ & $\mathbf{0 . 0 0 0 2}$ \\
\hline Percent Ash & $4.27 \pm 0.433$ & $4.03 \pm 0.552$ & 0.7394 \\
\hline Percent Non-Fiber Carbohydrate & $42.46 \pm 0.886$ & $32.84 \pm 1.327$ & $<\mathbf{0 . 0 0 0 1}$ \\
\hline $\begin{array}{l}\text { Percent Total Digestible } \\
\text { Nutrients }\end{array}$ & $72.12 \pm 0.552$ & $71.57 \pm 0.868$ & 0.5838 \\
\hline Net Energy for Gain & & $0.82 \pm 0.012$ & 0.5995 \\
\hline Net Energy for Maintenance & $0.83 \pm 0.007$ & $0.53 \pm 0.012$ & 0.5393 \\
\hline
\end{tabular}


Table 8: Fold change in cyclooxygenase 2, interleukin-10, nuclear factor kappa B, peroxisome proliferator-activated receptor gamma, and tumor necrosis alpha expression compared to the CP group in white blood cells in trial 1 of the supplement study. Data analyzed by ANOVA. Data for IL-10 and COX2 were log transformed before analysis. (FL ( $n=3)$ : flaxseed supplement + LPS, FP ( $n=3)$ : flaxseed supplement + PBS, CL ( $n=5)$ : control supplement + LPS, CP ( $n=5)$ : control supplement + PBS) Mean \pm SEM

\begin{tabular}{|c|c|c|c|c|c|}
\hline $\begin{array}{l}\text { Gene/Time } \\
\text { points }\end{array}$ & $\begin{array}{l}\text { Treatment } \\
\text { Group }\end{array}$ & Mean \pm SEM & $\begin{array}{l}\text { Supplement } \\
\text { Effect }\end{array}$ & LPS Effect & $\begin{array}{l}\text { Supplement } \\
\text { x LPS Effect }\end{array}$ \\
\hline COX-2 & & & $\mathrm{p}=0.27$ & $p=0.15$ & $\mathrm{p}=0.18$ \\
\hline \multirow{4}{*}{$\begin{array}{l}0, .5,1, \\
1.5,2.5,3 \\
\text { hrs }\end{array}$} & FL & $2.37 \pm 0.65$ & & & \\
\hline & FP & $2.65 \pm 0.63$ & & & \\
\hline & $\mathrm{CL}$ & $1.86 \pm 0.74$ & & & \\
\hline & $\mathrm{CP}$ & $1 \pm 0$ & & & \\
\hline IL-10 & & & $p=0.08$ & $p=0.43$ & $p=0.88$ \\
\hline \multirow{4}{*}{$\begin{array}{l}0,1.5,3,4 \\
5,6,10 \mathrm{hrs}\end{array}$} & FL & $2.82 \pm 0.96$ & & & \\
\hline & FP & $2.07 \pm 0.96$ & & & \\
\hline & $\mathrm{CL}$ & $1.67 \pm 0.61$ & & & \\
\hline & $\mathrm{CP}$ & $1 \pm 0$ & & & \\
\hline NF-кB & & & $\mathrm{p}=0.90$ & $\mathrm{p}=0.47$ & $\mathrm{p}=0.13$ \\
\hline \multirow{4}{*}{$\begin{array}{l}0,1.5,2.5,3, \\
4,5,6,7,9, \\
10,12,24 \\
\text { hrs }\end{array}$} & FL & $1.38 \pm 0.43$ & & & \\
\hline & FP & $1.92 \pm 0.39$ & & & \\
\hline & $\mathrm{CL}$ & $2.46 \pm 1.15$ & & & \\
\hline & $\mathrm{CP}$ & $1 \pm 0$ & & & \\
\hline PPAR $\gamma$ & & & $\mathrm{p}=0.45$ & $p=0.17$ & $\mathrm{p}=0.07$ \\
\hline \multirow{4}{*}{$\begin{array}{l}0,0.5,1, \\
1.5,3,5,6, \\
7,8,9,10, \\
11,12,24 \\
\text { hrs }\end{array}$} & FL & $0.65 \pm 0.16$ & & & \\
\hline & FP & $1.18 \pm 0.16$ & & & \\
\hline & $\mathrm{CL}$ & $1.07 \pm 0.24$ & & & \\
\hline & $\mathrm{CP}$ & $1 \pm 0$ & & & \\
\hline TNFa & & & $\mathrm{p}=0.57$ & $\mathrm{p}=0.35$ & $\mathrm{p}=0.35$ \\
\hline \multirow{4}{*}{$\begin{array}{l}.5,1,1.5, \\
2.5,3,4,5, \\
6 \mathrm{hrs}\end{array}$} & FL & $1.6 \pm 0.43$ & & & \\
\hline & FP & $1.6 \pm 0.43$ & & & \\
\hline & CL & $1.74 \pm 0.52$ & & & \\
\hline & $\mathrm{CP}$ & $1 \pm 0$ & & & \\
\hline
\end{tabular}


Table 9: Fold change in interferon gamma, interleukin-6, interleukin-10, nuclear factor kappa B, and tumor necrosis alpha gene expression to LPS or PBS in early pregnant ewes fed a whole flaxseed or control supplement (supplement study trial 2). Data expressed as the fold change in gene expression using the $2^{-\Delta \Delta \mathrm{Ct}}$ method with GAPDH as the housekeeping gene and the reference time point being the sample collected at CIDR withdraw. Data analyzed by repeated measures ANOVA. Mean \pm SEM. Mean is the overall mean of the fold change across the time points but analyzed with time as the repeated measure. (FL ( $\mathrm{n}=11)$ : flaxseed supplement + LPS; FP ( $\mathrm{n}=10)$ : flaxseed supplement + PBS; CL ( $\mathrm{n}=11)$ : control supplement + LPS; CP ( $n=10)$ : control supplement + PBS)

\begin{tabular}{|c|c|c|c|c|c|c|c|c|c|}
\hline $\begin{array}{l}\text { Gene/Time } \\
\text { points }\end{array}$ & $\begin{array}{l}\text { Treat } \\
\text { ment } \\
\text { Group }\end{array}$ & $\begin{array}{l}\text { Mean } \pm \\
\text { SEM }\end{array}$ & $\begin{array}{l}\text { Supple } \\
\text { ment } \\
\text { Effect }\end{array}$ & $\begin{array}{l}\text { LPS } \\
\text { Effect }\end{array}$ & $\begin{array}{l}\text { Supplem } \\
\text { ent } x \\
\text { LPS } \\
\text { Effect }\end{array}$ & $\begin{array}{l}\text { Hour } \\
\text { Effect }\end{array}$ & $\begin{array}{l}\text { Supplem } \\
\text { ent } x \\
\text { Hour }\end{array}$ & $\begin{array}{l}\text { LPS x } \\
\text { Hour }\end{array}$ & $\begin{array}{l}\text { Suppleme } \\
\text { nt x LPS } \\
\text { x Hour }\end{array}$ \\
\hline IFN $\gamma$ & & & $\mathrm{p}=0.75$ & $\mathrm{p}=0.29$ & $\mathrm{p}=0.56$ & $\mathrm{p}=0.13$ & $\mathrm{p}=0.96$ & $\mathrm{p}=0.51$ & $\mathrm{p}=0.52$ \\
\hline \multirow{4}{*}{$\begin{array}{l}0,0.5,1.5, \\
3,6,9,12, \\
24 \mathrm{hrs}\end{array}$} & FL & $0.82 \pm 0.27$ & & & & & & & \\
\hline & FP & $1.09 \pm 0.19$ & & & & & & & \\
\hline & $\mathrm{CL}$ & $0.66 \pm 0.13$ & & & & & & & \\
\hline & $\mathrm{CP}$ & $3.92 \pm 1.15$ & & & & & & & \\
\hline IL-6 & & & $\mathrm{p}=0.27$ & $\mathrm{p}=0.22$ & $\mathrm{p}=0.90$ & $\mathrm{p}=0.62$ & $\mathrm{p}=0.79$ & $\mathrm{p}=0.45$ & $\mathrm{p}=0.85$ \\
\hline \multirow{4}{*}{$\begin{array}{l}0,0.25, \\
0.75,1.5, \\
2,3,6 \mathrm{hrs}\end{array}$} & FL & $0.65 \pm 0.21$ & & & & & & & \\
\hline & FP & $0.84 \pm 0.32$ & & & & & & & \\
\hline & $\mathrm{CL}$ & $0.3 \pm 0.08$ & & & & & & & \\
\hline & $\mathrm{CP}$ & $0.61 \pm 0.14$ & & & & & & & \\
\hline IL-10 & & & $\mathrm{p}=0.96$ & $\mathrm{p}=0.28$ & $\mathrm{p}=0.86$ & $p=0.06$ & $\mathrm{p}=.99$ & $\mathrm{p}=.35$ & $p=0.36$ \\
\hline \multirow{4}{*}{$\begin{array}{l}0,1.5,3,6 \\
9,12 \mathrm{hrs}\end{array}$} & FL & $9.52 \pm 3.21$ & & & & & & & \\
\hline & FP & $4.47 \pm 1.73$ & & & & & & & \\
\hline & $\mathrm{CL}$ & $8.4 \pm 2.91$ & & & & & & & \\
\hline & $\mathrm{CP}$ & $3.7 \pm 0.89$ & & & & & & & \\
\hline NF-кB & & & $\mathrm{p}=0.24$ & $\mathrm{p}=0.60$ & $\mathrm{p}=0.90$ & $\mathrm{p}=0.54$ & $\mathrm{p}=0.54$ & $\mathrm{p}=0.46$ & $\mathrm{p}=0.06$ \\
\hline \multirow{4}{*}{$\begin{array}{l}0,1.5,3,6, \\
9 \mathrm{hrs}\end{array}$} & FL & $0.82 \pm 0.39$ & & & & & & & \\
\hline & FP & $1.05 \pm 0.24$ & & & & & & & \\
\hline & $\mathrm{CL}$ & $0.58 \pm 0.14$ & & & & & & & \\
\hline & $\mathrm{CP}$ & $0.75 \pm 0.18$ & & & & & & & \\
\hline TNFa & & & $\mathrm{p}=0.16$ & $\mathrm{p}=0.60$ & $\mathrm{p}=0.71$ & $\mathrm{p}=0.22$ & $\mathrm{p}=0.79$ & $p=0.26$ & $\mathrm{p}=0.21$ \\
\hline \multirow[t]{4}{*}{$0,0.5,1 \mathrm{hr}$} & FL & $1.1 \pm 0.20$ & & & & & & & \\
\hline & FP & $1.13 \pm 0.31$ & & & & & & & \\
\hline & $\mathrm{CL}$ & $0.78 \pm 0.14$ & & & & & & & \\
\hline & $\mathrm{CP}$ & $0.53 \pm 0.09$ & & & & & & & \\
\hline
\end{tabular}




\section{Figures}

Trial 1: The effect of supplementing with $\omega-3$ PUFA on LPS challenge in early pregnant Dorset ewes

\section{Rectal Temperatures}

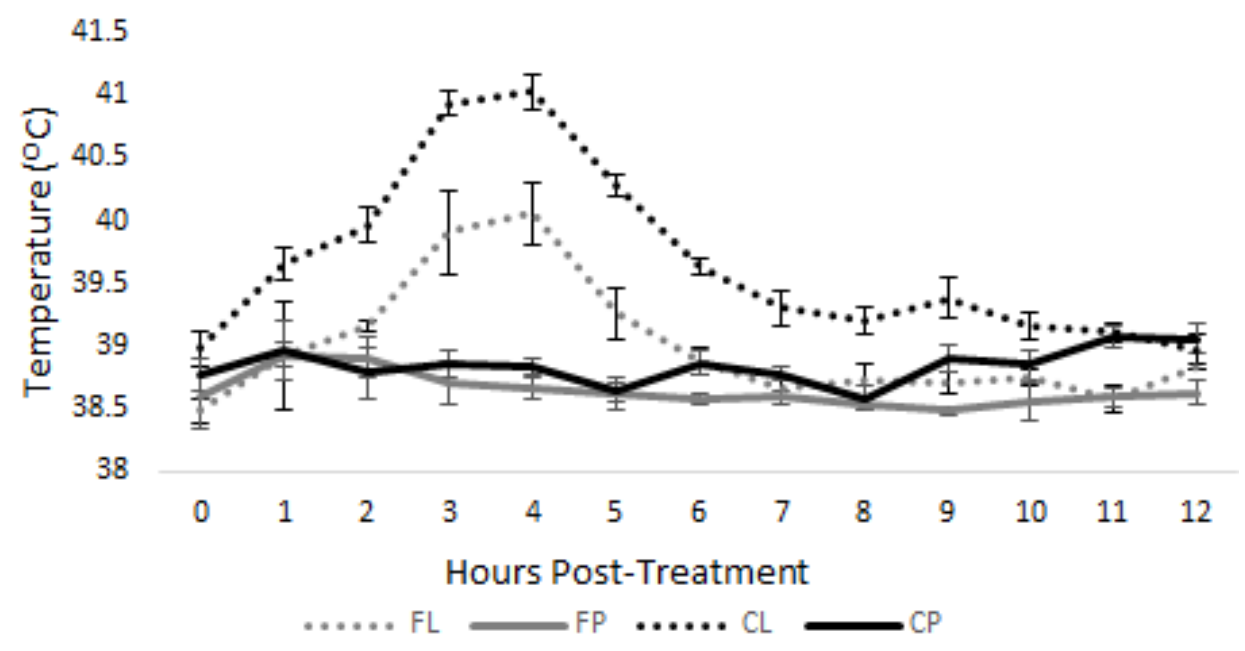

Figure 2: Hourly response in rectal temperature to LPS or PBS in early pregnant ewes fed a whole flaxseed or control supplement. Flax $\mathbf{p}<\mathbf{0 . 0 0 1}$, LPS P $<0.001$, Flax $x$ LPS $\mathbf{p}=\mathbf{0 . 0 0 6 4}$, Hour $\mathbf{p}<0.001$, LPS $\times$ Hour $\mathbf{p}<0.001$ (FL $(n=3)$ : flaxseed supplement + LPS, FP ( $n=3)$ : flaxseed supplement + PBS, CL $(n=5)$ : control supplement + LPS, CP $(n=5)$ : control supplement + PBS) Data were analyzed by repeated Measures ANOVA. Mean \pm SEM 


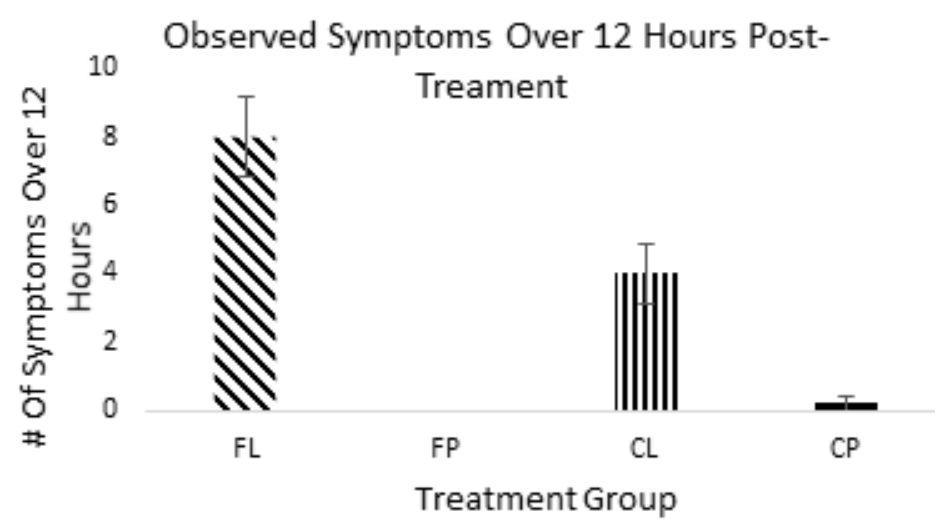

Figure 3: Frequency of sickness symptoms displayed by flaxseed and control supplement fed ewes receiving either LPS or PBS. Changes in behavior or mucosal responses were noted at each blood sample collection, added up for each ewe over the 12 hours post-treatment, and averaged for each treatment group. LPS $\mathbf{p}<\mathbf{0 . 0 0 0 1}(\mathrm{FL}(\mathrm{n}=3)$ : flaxseed supplement + LPS, FP (n=3): flaxseed supplement + PBS, CL ( $=5)$ : control supplement + LPS, CP ( $n=5)$ : control supplement + PBS). Data analyzed by ANOVA. Mean \pm SEM

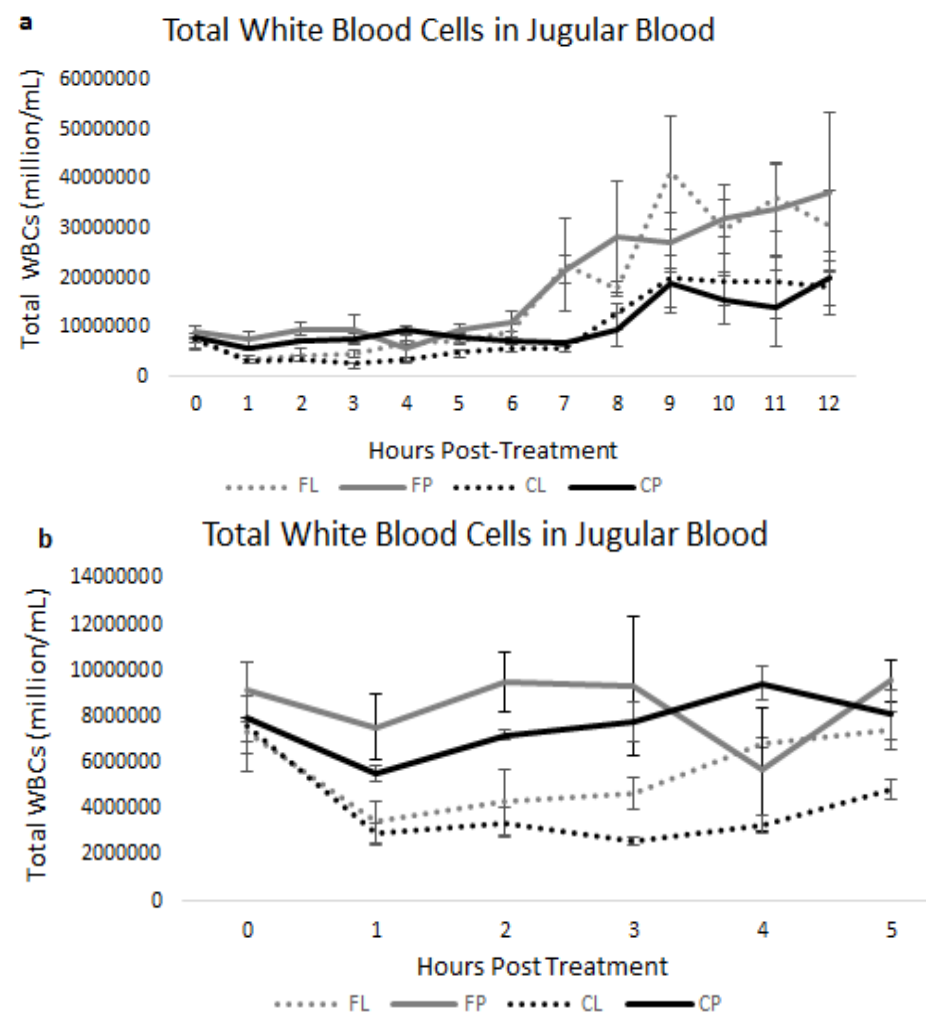

Figure 4: Hourly response in white blood cells to LPS or PBS in early pregnant ewes fed a whole flaxseed or control supplement from 0-12 hours (a) and 0-5 hours (b) post treatment. Figure a: Flax $\mathbf{p}<\mathbf{0 . 0 0 0 1}$, Hour $\mathbf{p}<\mathbf{0 . 0 0 0 1}$, Figure b: Flax $p=0.0157$, LPS $p<0.001$, Hour $p=0.0039$, LPS $x$ Hour $p=0.0054$, Flax $x$ LPS $x$ Hour $p=0.0056$ (FL ( $n=3)$ : flaxseed supplement + LPS, FP ( $n=3)$ : flaxseed supplement + PBS, CL ( $\mathrm{n}=5)$ : control supplement + LPS, $\mathrm{CP}(\mathrm{n}=5)$ : control supplement + PBS). Data were analyzed by repeated measures ANOVA. Mean \pm SEM 

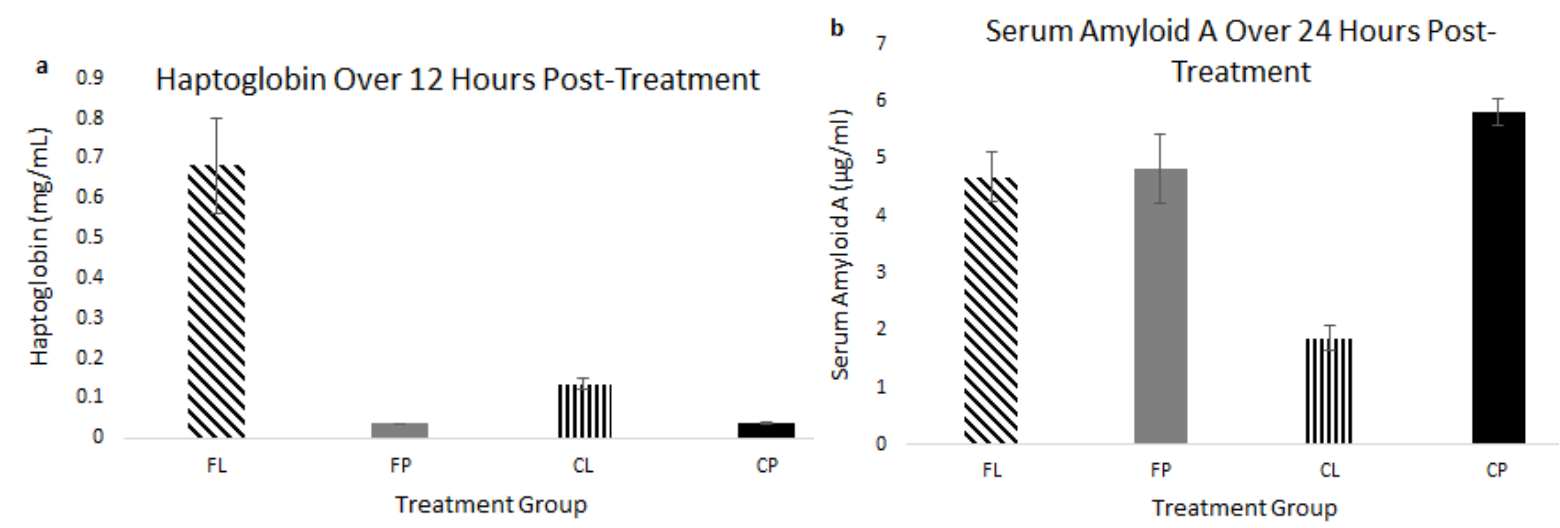

Figure 5: Plasma concentration of acute phase proteins post treatment. Haptoglobin (a) was measured in samples collected at time points 0,30 minutes, 1, 1.5, 2, 2.5, 3,6,9, and 12 hours. Data was log transformed before analysis; graph displays untransformed data. Both FL and CL are significantly different from the other 3 groups. Flax $\mathbf{p}<\mathbf{0 . 0 0 0 1}$, LPS $\mathbf{p}<\mathbf{0 . 0 0 0 1}$, Flax $x$ LPS $\mathbf{p}<\mathbf{0 . 0 0 0 1}$. Serum amyloid A (b) was measured in samples collected at time points 0,30 minutes, $1,1.5,2,2.5,3,6,9,12$, and 24 hours. CL had significantly lower SAA concentration compared to the other 3 groups. Flax $\mathbf{p}=\mathbf{0 . 0 2 1 6}$, LPS $\mathbf{p}<\mathbf{0 . 0 0 0 1}$, Flax $\mathbf{x}$ LPS $\mathbf{p}<\mathbf{0 . 0 0 1}$ (FL ( $\mathrm{n}=3$ ): flaxseed supplement + LPS; FP $(n=3)$ : flaxseed supplement + PBS, CL ( $n=5)$ : control supplement + LPS, CP ( $n=5)$ : control supplement + PBS) Data were analyzed by ANOVA with Tukey's HSD. Mean \pm SEM

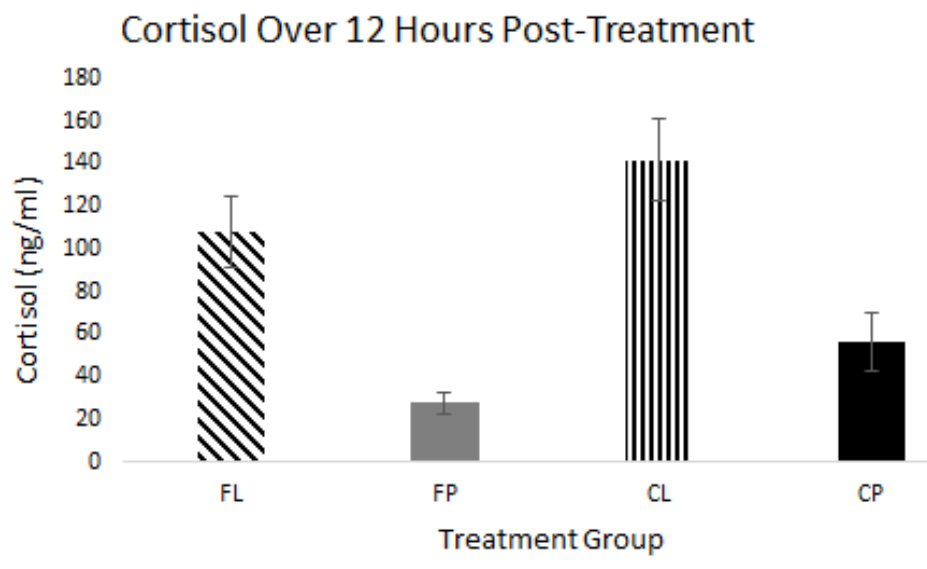

Figure 6: Plasma concentration of cortisol post treatment. Cortisol was measured in samples collected at time points 0,30 minutes, 1, 1.5, 2, 2.5, 3,6,9, and 12 hours. Flax p=0.0468, LPS p<0.001 (FL ( $\mathrm{n}=3$ ): flaxseed supplement + LPS, FP ( $n=3)$ : flaxseed supplement + PBS, CL ( $n=5)$ : control supplement + LPS, CP $(n=5)$ : control supplement + PBS) Data were analyzed by ANOVA. Mean \pm SEM 


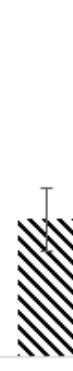

$\mathrm{FL}$

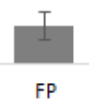

Treatment Group

c Interferon gamma Expression Over 24 Hours

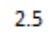

Post-Treatment
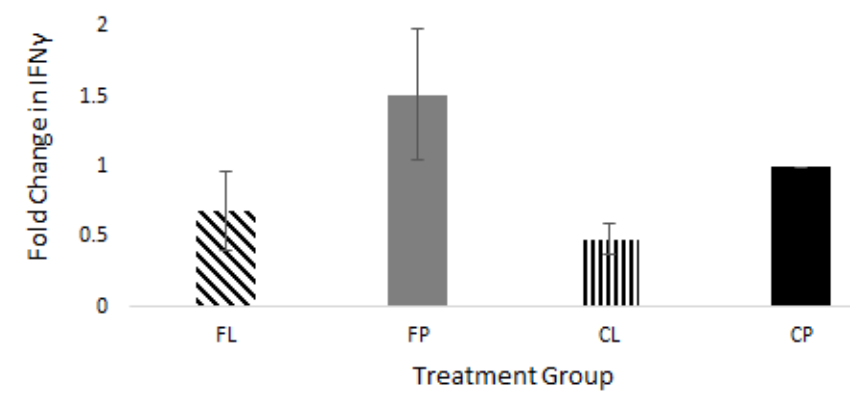

e Complement Component 3 Expression From 0-6

6

Hours Post-Treatment

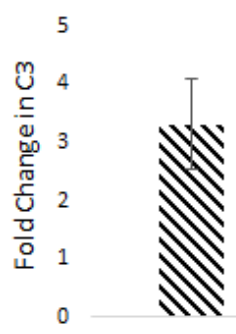

$\mathrm{FL}$

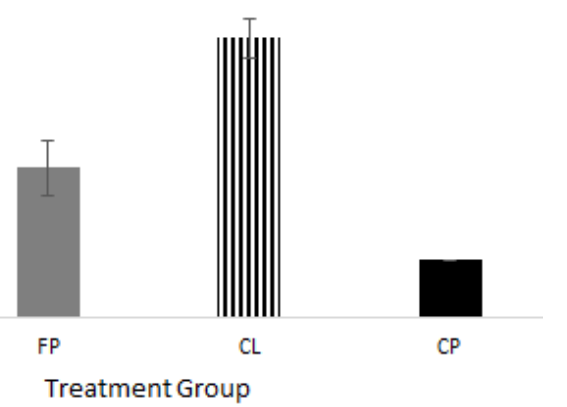

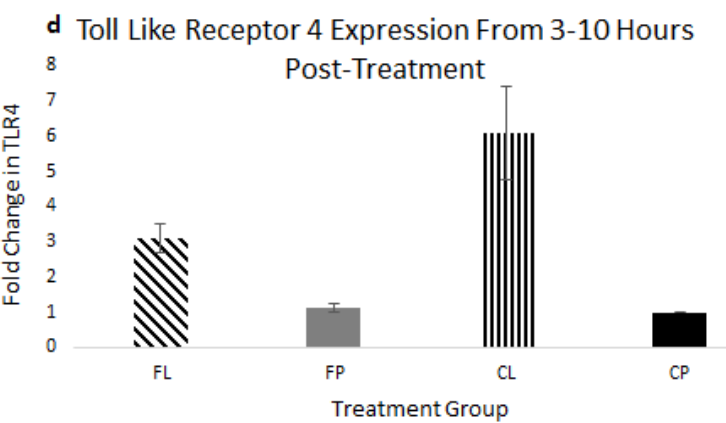

Figure 7: Fold change in gene expression of pro-inflammatory factors measured in white blood cells. Ct values were normalized to GAPDH and $\Delta \Delta \mathrm{Ct}$ values were obtained in reference to the CP group. (a) CXCL8 measured at 3, 4, 5, 6, 7, 8 hours post treatment. LPS $\mathbf{p}<\mathbf{0 . 0 0 0 1}$, Flax p=0.0486, Flax $\mathbf{x}$ LPS p=0.027 (b) IL-6 measured at 5, 1, 1.5, 2.5, 3, 4, 5, 6 hours post treatment. Flax x LPS p=0.0296 (c) IFN $\gamma$ measured at $0, .5,1,1.5,4,6,8,10,12,24$ hours post treatment. Data log transformed before analysis; graph displays untransformed data. LPS=0.0024 (d) TLR4 measured at 3, 4, 5, 6, 7, 8, 9, 10 hours post treatment. Flax $\mathbf{p}=\mathbf{0 . 0 4 8 4}$, LPS $<0.0001$, Flax $x$ LPS p=0.0326 (e) C3 measureed at collected $0, .5,1,1.5,2.5,3,4,5,6$ hours post treatment. Data log transformed before analysis; graph displays untransformed data. LPS=0.0065 (FL $(n=3)$ : flaxseed supplement + LPS, FP $(n=3)$ : flaxseed supplement + PBS, CL ( $n=5)$ : control supplement + LPS, CP ( $n=5)$ : control supplement + PBS) Data were analyzed by ANOVA. Mean \pm SEM 

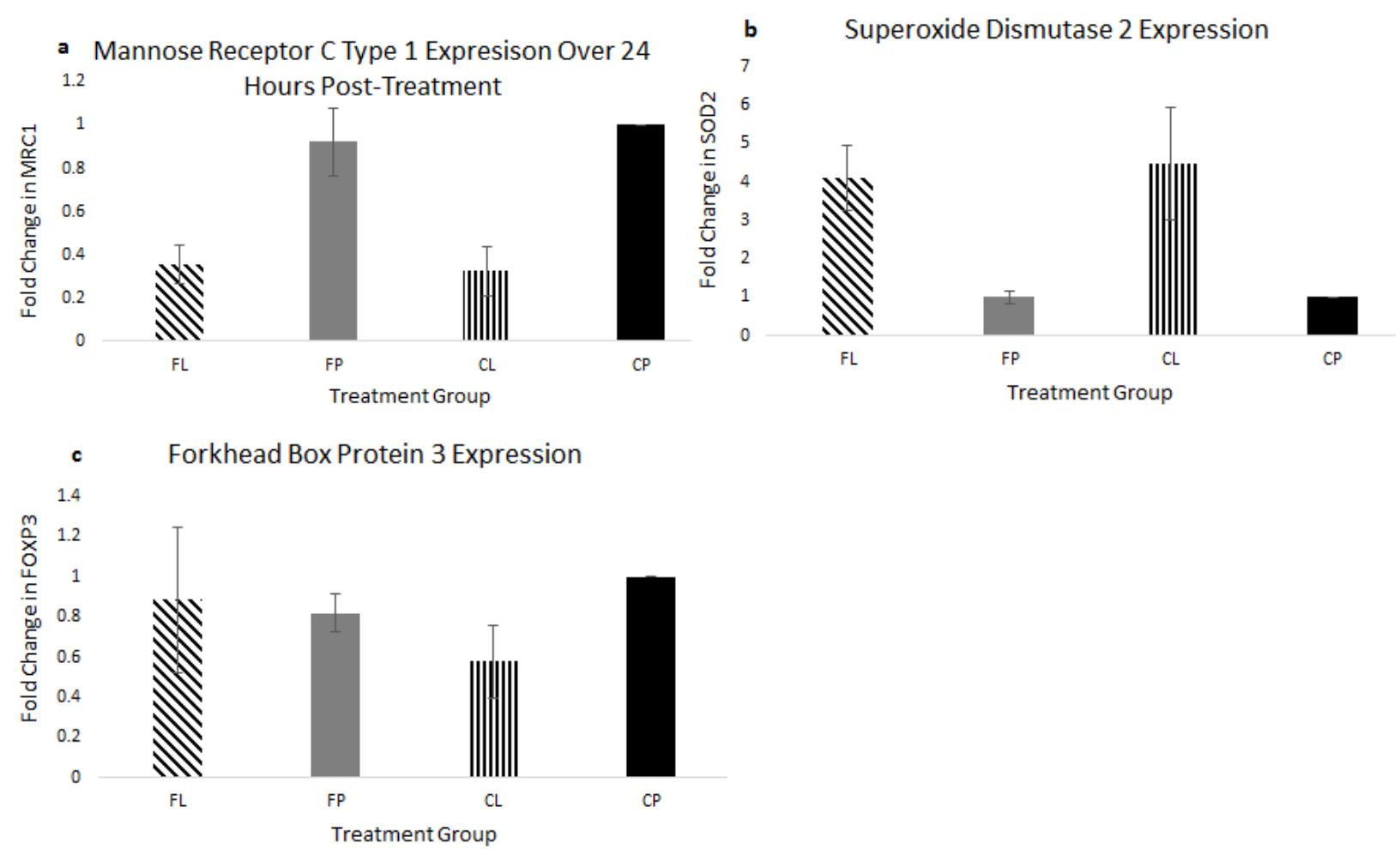

Figure 8: Fold change in gene expression of anti-inflammatory factors measured in white blood cells. Ct values were normalized to GAPDH and $\Delta \Delta \mathrm{Ct}$ values were obtained in reference to the CP group. (a) MRC1 measured at .5, 1, 1.5, 2.5, 6, 7, 8, 9, 10, 11, 12, 24 hours post treatment. LPS p<0.0001 (b) SOD2 measured in samples collected at $1.5,2.5,3,4,7,9,11,12,24$ hours post treatment. Data log transformed before analysis; graph displays

untransformed data LPS p<0.0001 (c) Foxp3 measured in samples collected at 1,1.5,3,4,6,7,8,9,10,11,12,24 hours post treatment and on d16 \& d25 post coitus. Data log transformed before analysis; graph displays untransformed data. LPS p=0.0259 (FL ( $n=3)$ : flaxseed supplement + LPS; FP ( $n=3)$ : flaxseed supplement + PBS; CL ( $=5)$ : control supplement + LPS, CP $(n=5)$ : control supplement + PBS) Data were analyzed by ANOVA. Mean \pm SEM 


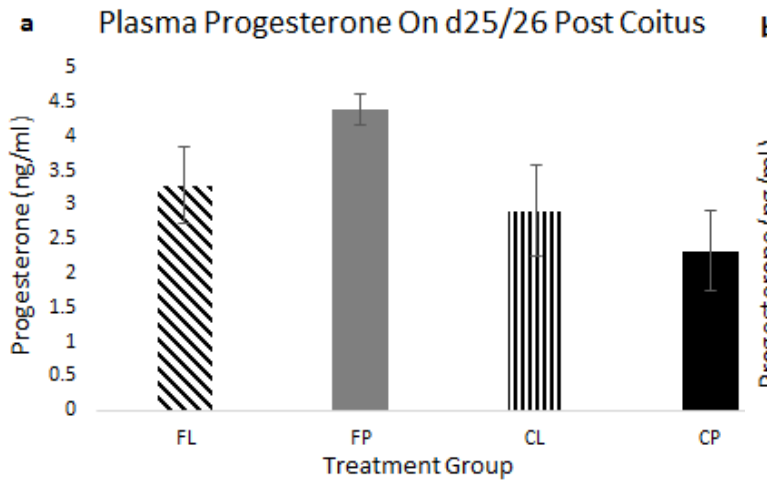

Only

Figure 9: Plasma progesterone levels. (a) Progesterone on day 25/26 post coitus comparing all 4 treatment groups. Data analyzed by 2-way ANOVA. Flax $p=0.0752$, LPS $\mathrm{p}=0.6854$, Flax $x$ LPS $\mathrm{p}=0.2026$ (FL (n=3): flaxseed supplement + LPS, FP (n=3): flaxseed supplement + PBS, CL ( $n=5)$ : control supplement + LPS, CP ( $n=5)$ : control supplement + PBS) (b) Progesterone on day 25/26 post coitus comparing only between supplements. Data was analyzed by a $T$-test $\mathbf{p}=\mathbf{0 . 0 4 8 1}$ (flaxseed $n=6$, control $n=10)$ (FL ( $n=3)$ : flaxseed supplement + LPS, FP $(n=3)$ : flaxseed supplement + PBS, CL ( $n=5)$ : control supplement + LPS, CP ( $n=5)$ : control supplement + PBS) Mean \pm SEM

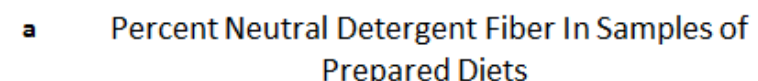

b Average Percent Non-Fiber Carbohydrate In
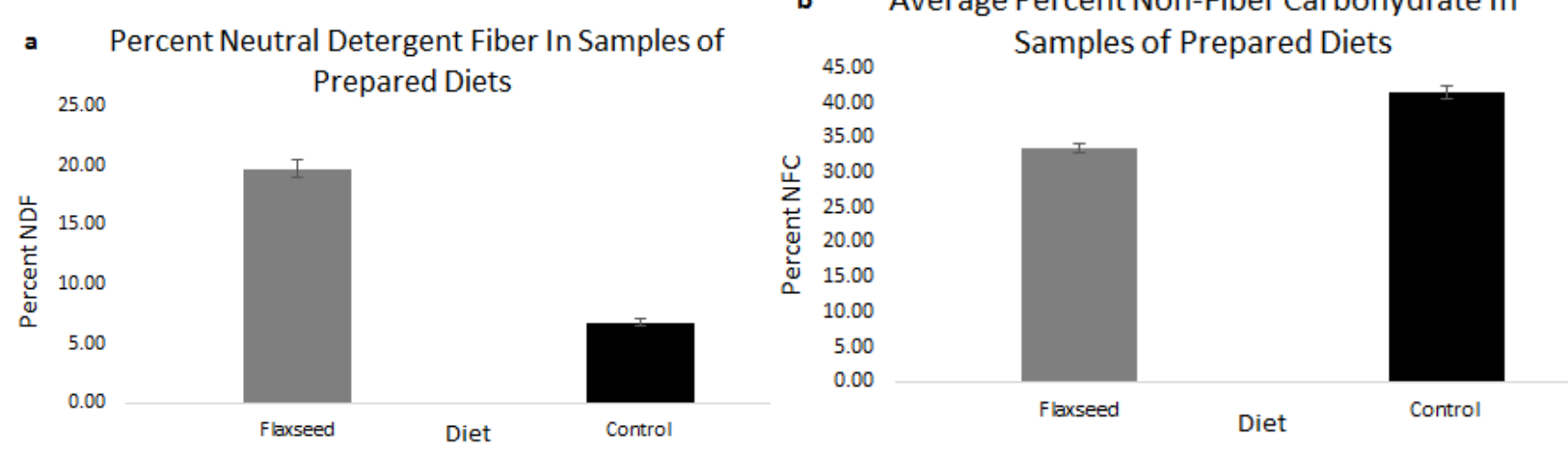

Figure 10: Comparison of the neutral detergent fiber (a) and non-fiber carbohydrate (b) in samples of the prepared supplements. Samples were analyzed by the Rumen Fermentation Profiling Laboratory at WVU. Data analyzed by T-test with Bonferroni correction with $\alpha=0.005$. NDF: $\mathbf{p}=\mathbf{0 . 0 0 0 1}, \mathbf{N F C}: \mathbf{p}=\mathbf{0 . 0 0 0 5}$ (flaxseed supplement samples $\mathrm{n}=4$; control supplement samples $\mathrm{n}=4$ ) Mean \pm SEM 
Trial 2: the effect of supplementing with $\omega-3$ PUFA on LPS challenge in early pregnant ewes

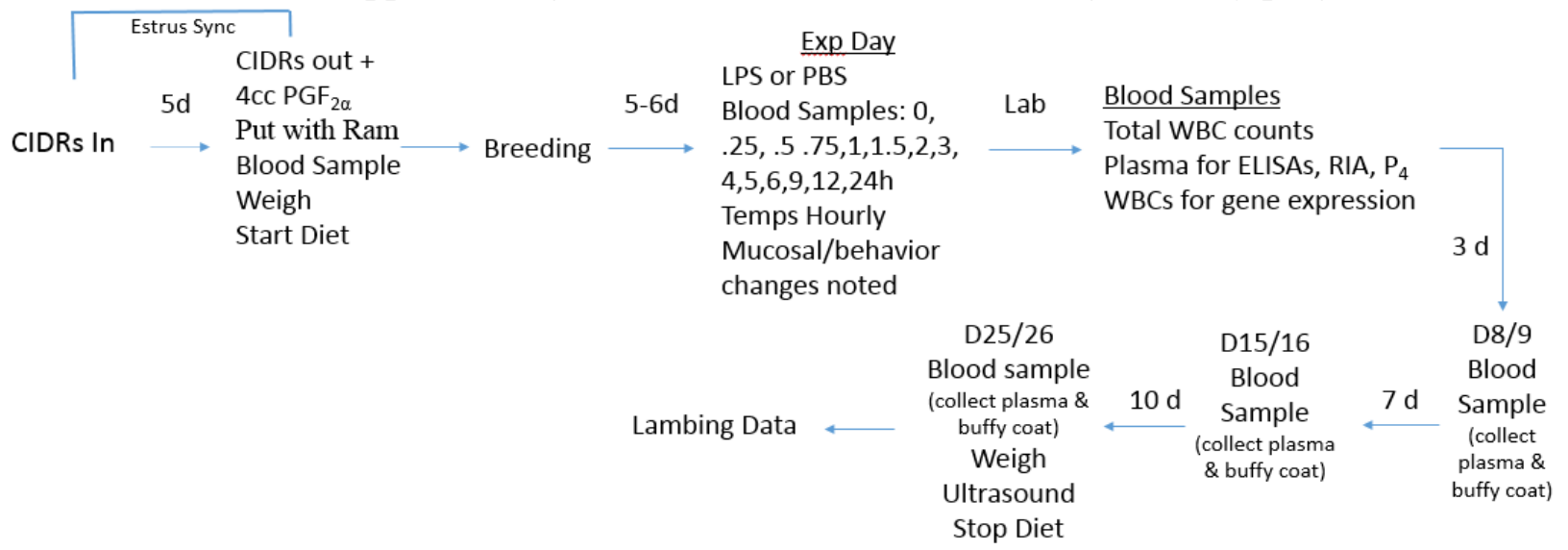

Figure 11: Timeline outlining the steps in the breed effects and the omega-3 PUFA effect on LPS challenge studies conducted in the Fall 2015.
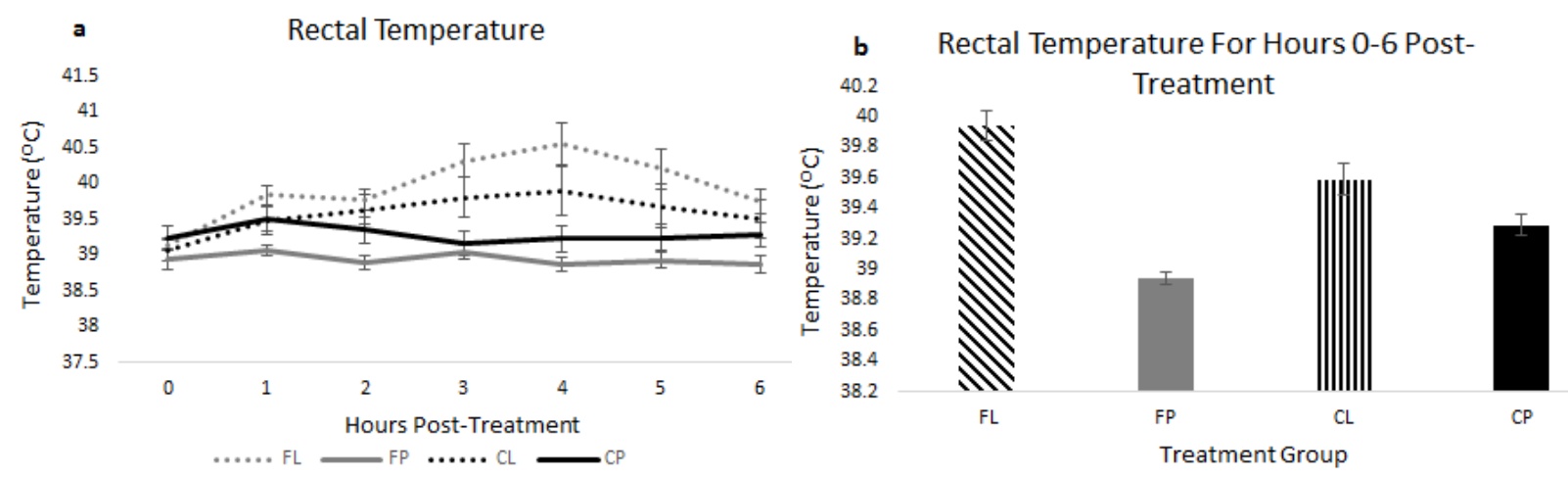

Figure 12: Rectal temperature to LPS or PBS in early pregnant ewes fed a whole flaxseed or control supplement. (a) Hourly response in rectal temperature. Data analyzed by repeated Measures ANOVA. LPS p $<\mathbf{0 . 0 0 0 1 , ~ F l a x ~ x ~ L P S ~}$ $\mathbf{p}=\mathbf{0 . 0 1 6 4}$, Hour $\mathbf{p}<\mathbf{0 . 0 0 0 1}$ (b) Average rectal temperature for the first six hours post treatment. Data were analyzed by ANOVA. LPS p $<\mathbf{0 . 0 0 0 1 , ~ F l a x ~ x ~ L P S ~} \mathbf{p}<\mathbf{0 . 0 0 0 1}$ (FL ( $\mathrm{n}=11$ ): flaxseed supplement + LPS, FP ( $\mathrm{n}=10)$ : flaxseed supplement + PBS, CL ( $n=11)$ : control supplement + LPS, CP ( $n=10):$ control supplement + PBS) 


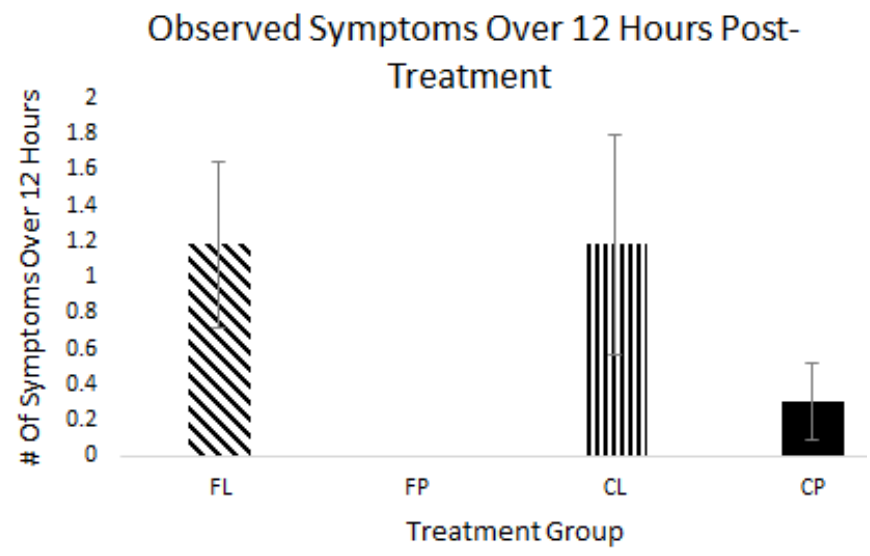

Figure 13: Frequency of sickness symptoms displayed by flaxseed and control supplement fed ewes receiving either LPS or PBS. Changes in behavior or noticeable sickness symptoms were noted at whole hour (hrs $0,1,2,3,4,5,6,9,12)$ blood sample collection times, added up for each ewe over the 12 hours post-treatment, and averaged for each treatment group. LPS p<0.0001 (FL $(n=11)$ : flaxseed supplement + LPS, FP ( $n=10)$ : flaxseed supplement + PBS; CL $(n=11)$ : control supplement + LPS, CP $(n=10)$ : control supplement + PBS) Data were analyzed by ANOVA Mean \pm SEM

a Total White Blood Cells in Jugular Blood

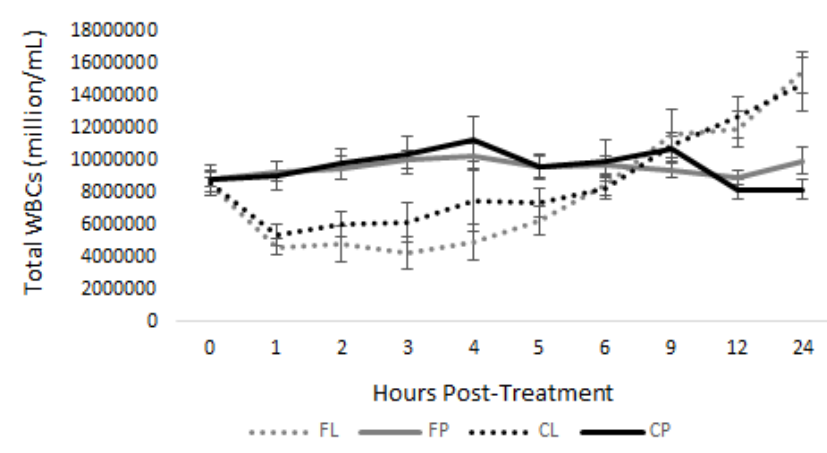

b Total White Blood Cells In Jugular Blood
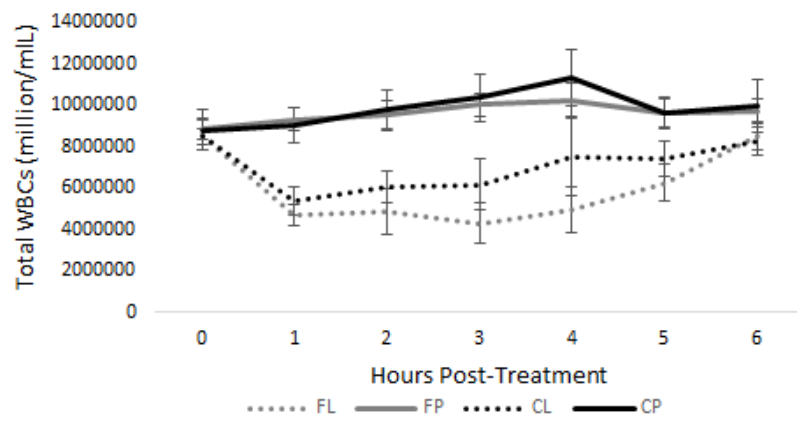

Figure 14: Response in white blood cells to LPS or PBS in early pregnant ewes fed a whole flaxseed or control supplement from (a) 0-24 hours post treatment. LPS p=0.0037 (b) 0-6 hours post treatment. LPS p<0.0001, Hour p=0.0004, LPS x Hour p<0.0001 (FL $(n=11)$ : flaxseed supplement + LPS, FP ( $n=10)$ : flaxseed supplement + PBS; CL $(n=11)$ : control supplement + LPS, CP $(n=10)$ : control supplement + PBS) Data were analyzed by repeated measures ANOVA. Mean \pm SEM 

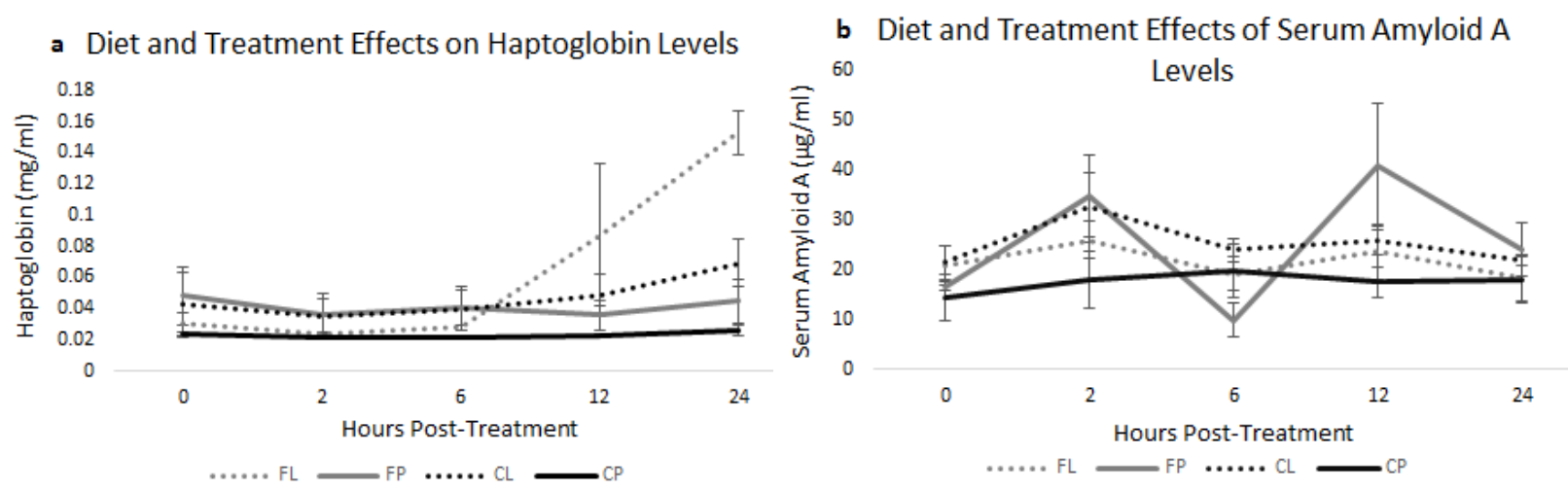

Figure 15: Acute phase proteins were measured in plasma at $0,2,6,12$, and 24 hours post-treatment. (a) Haptoglobin: LPS $\mathbf{p = 0 . 0 3 2 7}$, Hour $\mathbf{p}=\mathbf{0 . 0 0 7 8}$, LPS $\mathbf{x}$ Hour $\mathbf{p}=\mathbf{0 . 0 1 6 5}$ (b) Serum Amyloid A: Hour $\mathbf{p = 0 . 0 0 2 3}$ (FL $(n=11)$ : flaxseed supplement + LPS, FP $(n=10)$ : flaxseed supplement + PBS, CL ( $n=11)$ : control supplement + LPS, $\mathrm{CP}(\mathrm{n}=10)$ : control supplement + PBS) Data were analyzed by repeated measures ANOVA Mean \pm SEM

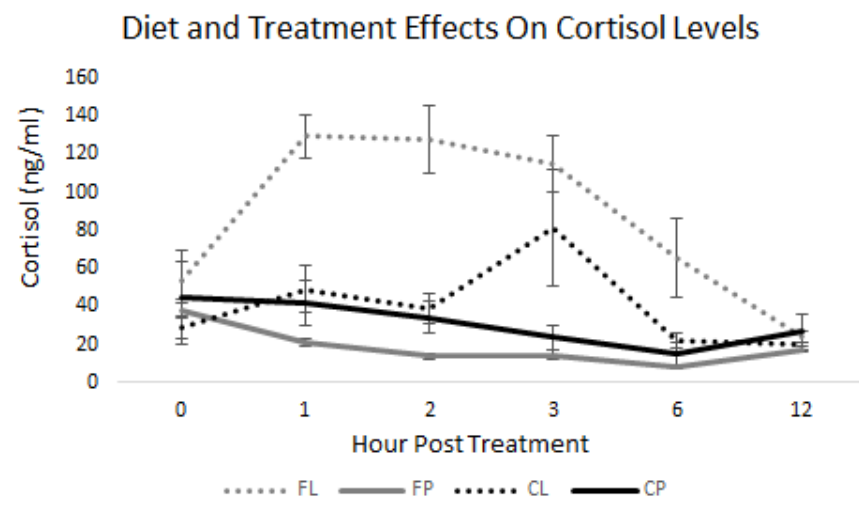

Figure 16: Cortisol was measured in plasma at $0,1,2,3,6$ and 12 hours post-treatment. Flax $\mathbf{p}=\mathbf{0 . 0 2 3 5}$, LPS $p<0.0001$, Flax $x$ LPS p=0.0007, Hour $p<0.0001$, LPS $x$ Hour $p<0.0001$, Flax $x$ LPS $x$ Hour $p=0.0105$ (FL $(\mathrm{n}=11)$ : flaxseed supplement + LPS, FP ( $\mathrm{n}=10)$ : flaxseed supplement + PBS, CL $(\mathrm{n}=11)$ : control supplement + LPS, $\mathrm{CP}(\mathrm{n}=10)$ : control supplement + PBS) Data were analyzed by repeated measures ANOVA Means \pm SEM 

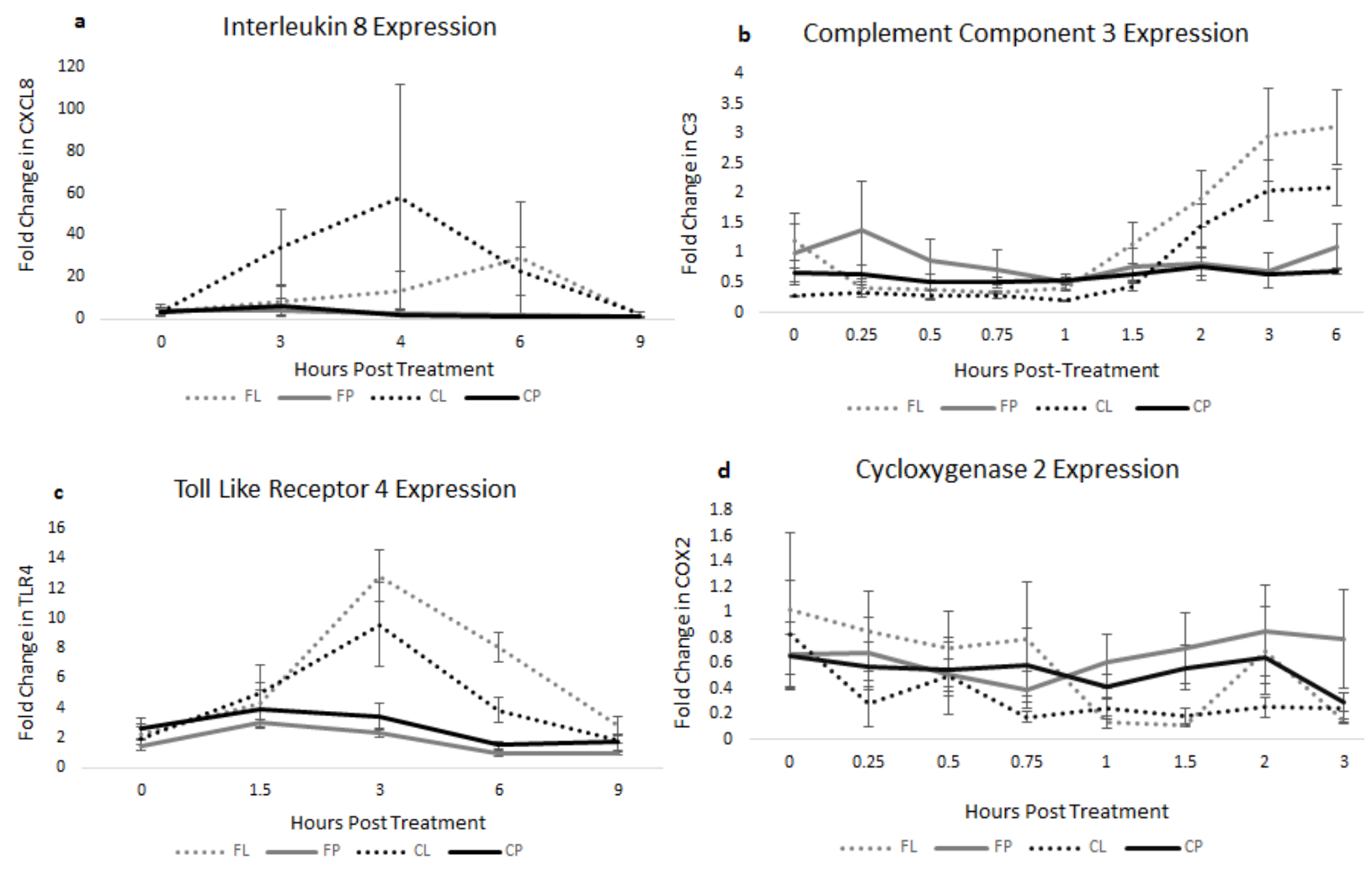

Figure 17: Fold change in gene expression of pro-inflammatory factors measured in white blood cells in response to LPS or PBS in early pregnant ewes fed a whole flaxseed or control supplement. (a) CXCL8: LPS p=0.0160 (b) C3: Flax $p=0.0041$, LPS $p=0.0107$, Hour $p<0.0001$, LPS $x$ Hour $p<0.0001$ (c) TLR4: LPS $p=0.0002$, Hour $p<0.0001$, LPS $\mathbf{x}$ Hour $\mathbf{p}<\mathbf{0 . 0 0 0 1}$ (d) COX2: Hour $\mathbf{p}=\mathbf{0 . 0 1 1 9}$ Gene Expression normalized to GAPDH and referenced to expression levels in the sample collected at CIDR withdraw. (FL $(n=11)$ : flaxseed supplement + LPS, FP ( $n=10)$ : flaxseed supplement + PBS, CL ( $n=11)$ : control supplement + LPS, CP $(n=10)$ : control supplement + PBS) Data were analyzed by repeated measures ANOVA. Mean \pm SEM 

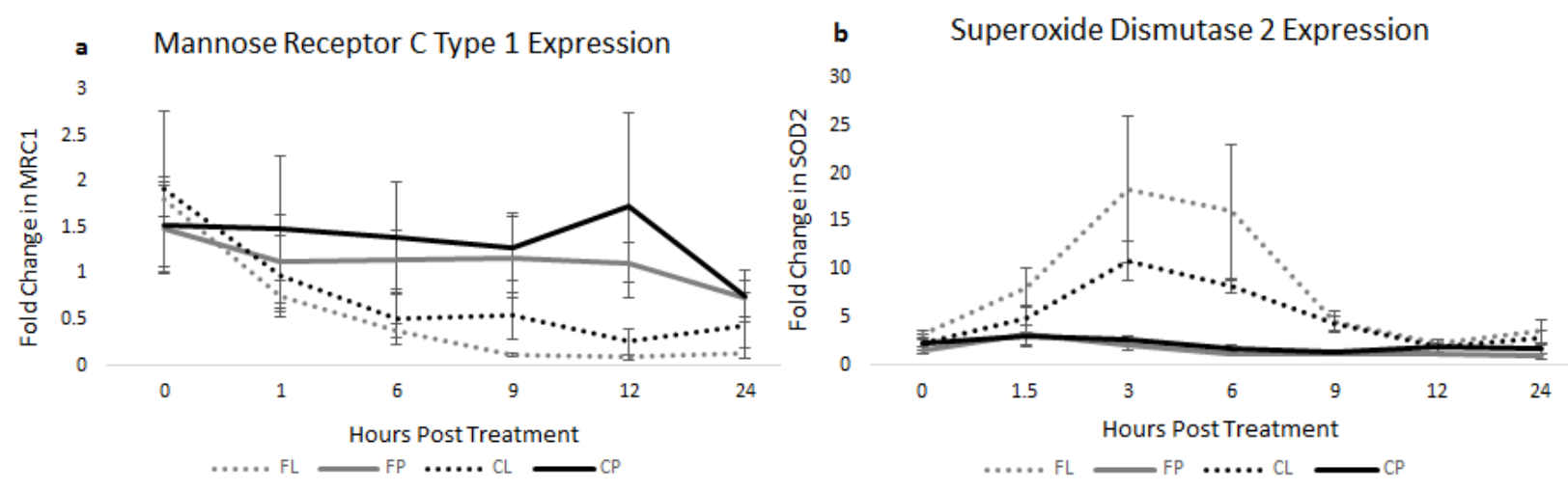

c Peroxisome Proliferator-Activated Receptor gamma Expression

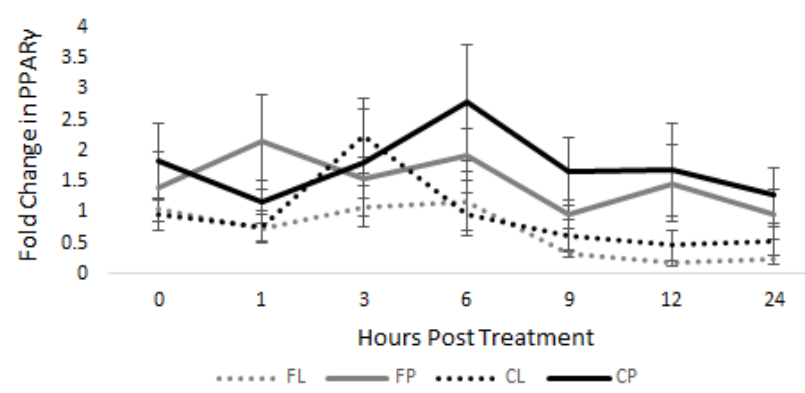

d Forkhead Box P3 Expression

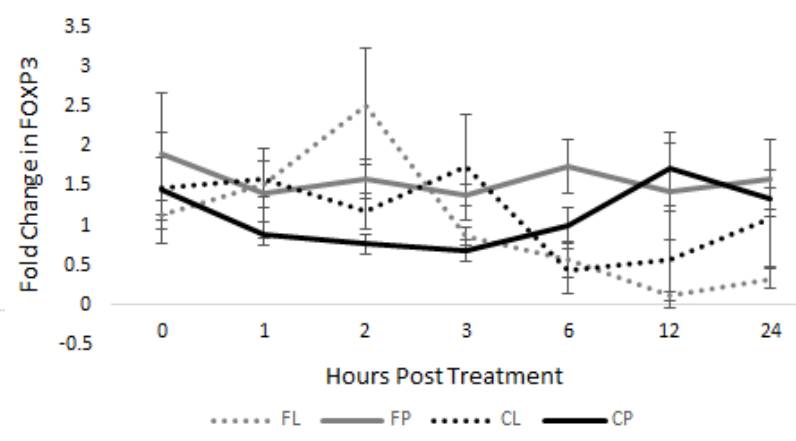

e Transforming Growth Factor beta Expression

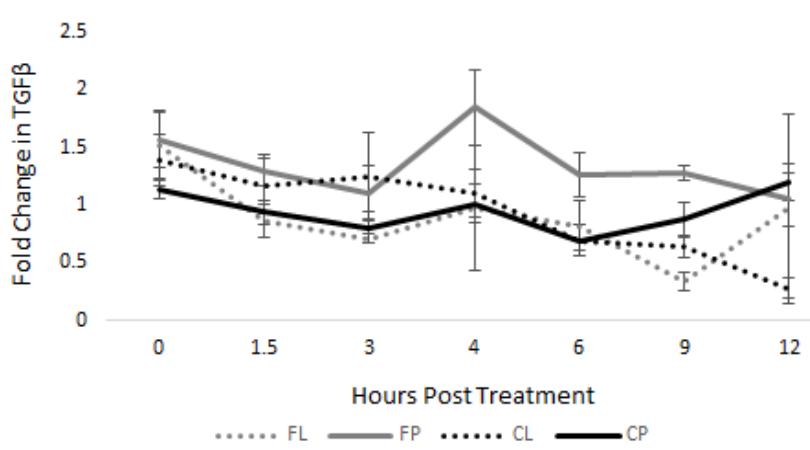

Figure 18: Fold change in gene expression of anti-inflammatory factors measured in white blood cells in response to LPS or PBS in early pregnant ewes fed a whole flaxseed or control supplement (a) MRC1: LPS p=0.0118, Hour p=0.0004, LPS x Hour p=0.0091 (b) SOD2: LPS p<0.0001, Hour p<0.0001, LPS x Hour p<0.0001. (c) PPAR $\gamma$ : LPS p=0.0014, Hour p=0.0436 (d) Foxp3: LPS x Hour p=0.0242 (e) TGF $\beta$ : Hour p=0.0117 (c) TLR4: LPS $\mathbf{p}=\mathbf{0 . 0 0 0 2}$, Hour $\mathbf{p}<\mathbf{0 . 0 0 0 1}$, LPS $\mathbf{x}$ Hour $\mathbf{p}<\mathbf{0 . 0 0 0 1}$ Gene Expression normalized to GAPDH and referenced to expression levels in the sample collected at CIDR withdraw. (FL $(n=11)$ : flaxseed supplement + LPS, FP ( $n=10)$ : flaxseed supplement + PBS, CL $(n=11)$ : control supplement + LPS, CP $(n=10)$ : control supplement + PBS $)$. Data were analyzed by repeated measures ANOVA. Mean \pm SEM 


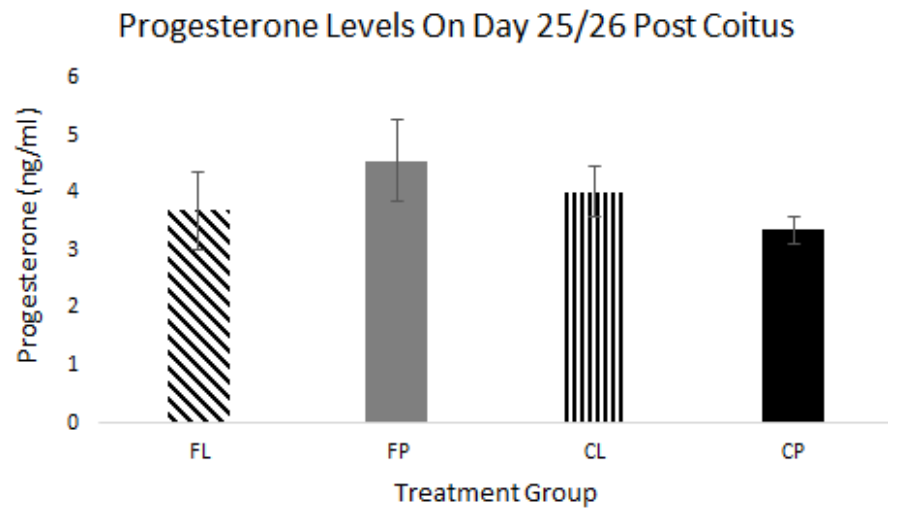

Figure 19: Plasma concentration of progesterone on day 25/26 post coitus in ewes fed flaxseed or a control supplement and administered LPS or PBS on day 5/6 post coitus. Data were analyzed by 2-way ANOVA. Flax $\mathrm{p}=0.4303$, LPS $\mathrm{p}=0.8523$, Flax x LPS p=0.1766 (FL $(n=11)$ : flaxseed supplement + LPS, FP ( $n=10)$ : flaxseed supplement + PBS, CL $(n=11)$ : control supplement + LPS, CP $(n=10)$ : control supplement + PBS $)$

a Percent Neutral Detergent Fiber in Diet Samples

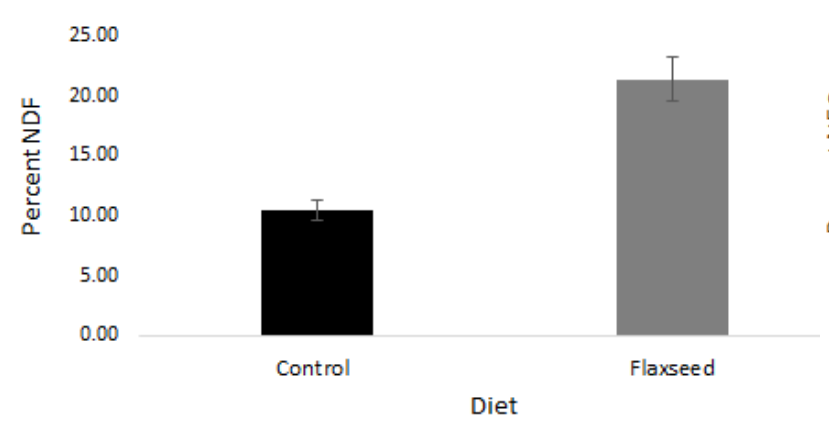

b Percent Non-Fiber Carbohydrate in Diet Samples

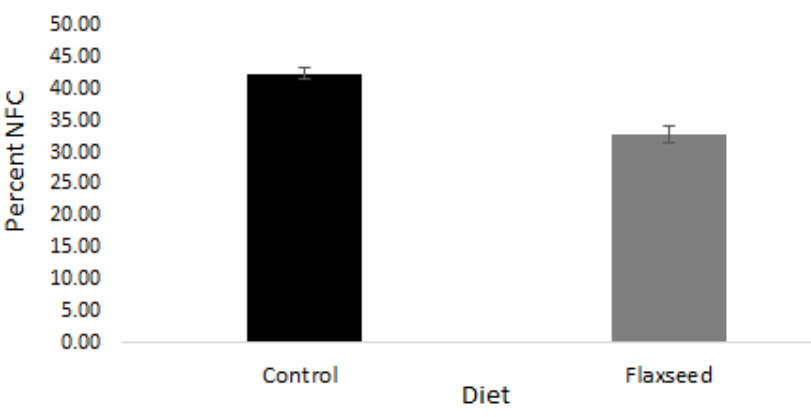

Figure 20: Comparison of the neutral detergent fiber (a) and non-fiber carbohydrate (b) in samples of the prepared supplement s. Samples were analyzed by the Rumen Fermentation Profiling Laboratory at WVU. Data were analyzed by $T$-test with Bonferroni correction with $\alpha=0.005$. NDF: $\mathbf{p}=\mathbf{0 . 0 0 0 2}, \mathbf{N F C :} \mathbf{p}<\mathbf{0 . 0 0 0 1}$ (flaxseed supplement samples $n=5$, control supplement samples $n=7$ ) Mean \pm SEM 


\section{Chapter 4: Effect of Breed on the LPS Response in Early Pregnant Ewes Introduction}

The fetus was once thought to be ignored by the maternal immune system due to physical separation, immunological immaturity of the fetus, and maternal immune inertness (Billington, 2003). It is now appreciated that a dynamic dialogue between maternal and fetal tissues has to occur to allow for immune tolerance of the fetus, angiogenesis and cytokine and hormonal balance (Dosiou \& Giudice, 2005; Thaxton \& Sharma, 2010; Sharma, 2014). During pregnancy, maternal immune cells are in contact with fetal trophoblast cells, which express paternal antigens and in order to have a successful pregnancy, the maternal immune system has to tolerate the semi-allogeneic fetus (Erlebacher, 2013; Sharma, 2014). The immune cells in the pregnant uterus that are in charge of this tolerance are tolerogenic DCs, M2 $\mathrm{M \Phi s}$, and regulatory T cells (Tregs). During pregnancy these cells are responsible for creating a microenvironment that is compatible with pregnancy and supports placentation (Sharma, 2014) including a shift towards T helper (Th) 2 cytokine dominance and inhibition of complement activation (Mellor et al., 2001; Girardi et al., 2006).

Infection is an important trigger for inflammation causing changes in the function of immune cells at the maternal-fetal interface which can cause embryonic loss (Aisemberg et al., 2013; Kwak-Kim et al., 2014; Sharma et al., 2014). Pattern recognition receptors at the maternal-fetal interface recognize PAMPs; binding of these receptors to pathogen molecules results in the activation of anti-pathogen responses, such as the synthesis and secretion of chemokines and PICs, often through the activation of transcription factors such as NF-kB and Ap-1(Akira et al., 2006; Koge et al., 2014; Gioia et al., 2015). Toll like receptors are one family of these innate immune system PRRs and TLR4 is the member that recognizes the gram negative bacterial cell wall component, LPS (Abrahams, 2008). Lipopolysaccharide triggers a robust inflammatory response characterized by the production of PICs, such as TNF $\alpha$ and IL$1 \beta$, shifts in the Th1/Th2 balance (Park et al., 2009) and induces uterine NK cell cytotoxic activation (Murphy et al., 2005). The immune mediators that are released stimulate cells to upregulate inflammation locally, while systemically they activate the HPA axis, cause the release of APPs, and induce the febrile response (Chrousos, 1992; 1995; Gabay \& Kushner, 1999; Kabaroff et al 2006). While this immune response is necessary to clear an infection, over activation of this pathway is detrimental to pregnancy and neutralization of LPS, or blockage of TLR4 signaling has been shown to prevent fetal loss in murine pregnancy (Friable et al., 2011). 
Early embryonic loss in response to bacterial infections can be due to several reasons; 1) direct embryotoxic effects of PIMs on the blastocyst, 2) apoptosis of blastocyst cells by PICs that hamper blastocyst growth and reduce IFNt production which will affect maternal recognition of pregnancy, the prevention of luteolysis, and P4 production, 3) luteolysis (direct action of PICs on the CL or through production of $\mathrm{PGF}_{2 \alpha}$ ) and loss of $\mathrm{P} 4$ production, ( $\mathrm{P} 4$ is immunosuppressive and needed to help generate maternal tolerance to the fetus in addition to regulating elongation and implantation genes), 4) reduced blastocyst size due to apoptosis by PICs and altered gene expression due to decreased IFNt and P4 can result in missing the "window of implantation," a time when the uterus is receptive to implantation and the blastocyst is implantation competent, 5) inflammation changing the normal spatial-temporal expression of cytokines involved in implantation, 6) and loss of maternal fetal tolerance. A highly proinflammatory uterine environment can cause the local immune cells to switch from a tolerogenic phenotype (tolerogenic DC, uterine NK cell, M2 MФ, Treg) to an immunogenic phenotype (immunogenic DC, peripheral NK cell, M1 MФ, effector T cell) that will no longer tolerate the presence of foreign paternal antigens.

After acquiring antigen, APCs travel to lymph nodes to present the antigen and activate T cells. If the APC is displaying a bacterial antigen, a Th1/pro-inflammatory response would be expected in order to clear the pathogen. If the APC was displaying a seminal plasma antigen, a Treg polarization is needed to acquire maternal tolerance to the fetus; failure to generate significant numbers of Treg cells specific to paternal antigens results in fetal loss (Robertson et al., 2013). A problem generating maternal tolerance could occur if there is a substantial uterine bacterial presence after mating resulting in predominantly Th1 polarization and infiltration of inflammatory cells into the uterus. This could result from failure of a dairy cow to efficiently deal with uterine contamination postpartum during NEB and/or by bacterial introduction during mating or AI. DCs are professional APC involved in the activation of T cells. Tolerogenic DCs have a specific immature or semi-immature phenotype that is pivotal in the activation and expansion of Treg cells (Steinman et al., 2003). Tolerogenic DCs have diminished expression of the co-stimulatory molecules CD80 and CD86; they do not express the Th1-polarizing cytokine IL-12 (Steinman et al., 2003) but do express IDO, which allows them to activate resting Tregs, convert CD4+CD25- T cells to CD4+CD25+FOXP3+ Tregs, and stimulate Tregs to maintain suppressive activity (Sharma et al., 2007). DCs that are differentiated in the presence of TGFß, IL-10, GM-CSF, and IL-4 are reported to behave like tolerogenic DCs and reliably induce CD4+CD25+ Tregs that have immunosuppressive function (Sato et al., 2003).

Estrogen is immune stimulatory. However, post-partum before ovarian activity resumes, estrogen is low which can predispose the uterus to bacterial colonization through loss of specific responses 
(antigen specific T helper cells), leaving the innate immune system to handle any bacterial contamination (Bondurant, 1999). Nonspecific responses may be compromised, however. When an LPS infusion was used to induce endometritis, the oxygen dependent mechanism (respiratory burst-generation of reactive oxygen species) of killing phagocytized bacteria by neutrophils was reduced (Kluciñski et al., 1995). Authors suggested that gram-negative bacterial contamination of the uterus postpartum compromised the ability of the first line of defense to control and clear pathogens. In addition, being in a state of NEB is immune suppressive and neutrophil function was reported to decline as parturition approached and DMI decreased and remained depressed after parturition in dairy cows for several weeks (Hammon et al., 2006). Kimura et al (1999) also reported that the onset of lactation as well as hypocalcemia (Kimura et al., 2006) reduced the bactericidal activity of neutrophils and the expression levels of adhesion molecules on neutrophils needed for the cells to enter tissues. For pathogens introduced during estrus, $\mathrm{E}_{2}$ levels are rapidly declining, which may allow the pathogen time to establish itself in the reproductive tract. Progesterone is immune suppressive, and any bacterial contamination present after ovulation could turn into an infection. Therefore, bacterial infections can increase the calving interval and impact lifetime milk production, obvious concerns for a producer.

The present study was conducted to gain information on the mechanism of bacterial infection induced embryonic loss using an LPS challenge in early pregnant ewes by measuring aspects of the innate immune response and APR. In a previous experiment at WVU, Dorset and Suffolk ewes appeared to have a differential response to LPS challenge. The objective in this study was to see if Dorset and Suffolk ewes responded to LPS differently in regard to rectal temperature,WBCs, observable symptoms, APPs, gene expression of cytokines, chemokines, receptors, and enzymes and whether pregnancy rates were associated with the inflammatory response.

\section{Results}

\section{Clinical Signs}

Temperature

Rectal temperature was affected by breed (Dorset $39.16 \pm 0.05^{\circ} \mathrm{C}$, Suffolk $39.45 \pm 0.06^{\circ} \mathrm{C}$, $\mathrm{p}=0.0284)$, LPS (LPS $39.54 \pm 0.06^{\circ} \mathrm{C}$, PBS $\left.38.99 \pm 0.04^{\circ} \mathrm{C}, \mathrm{p}=0.0001\right)$, hour $(\mathrm{p}<0.0001)$, and the interactions breed $\mathrm{x}$ hour $(\mathrm{p}=0.0330)$ and LPS $\mathrm{x}$ hour $(\mathrm{p}<0.0001)$ with the ewes receiving LPS having an increase in temperature from hour 0 (DL $39.08 \pm 0.15^{\circ} \mathrm{C}$, SL $38.6 \pm 0.08^{\circ} \mathrm{C}$ ) until hour 4 (DL39.89 \pm $0.34^{\circ} \mathrm{C}, \mathrm{SL} 40.11 \pm 0.19^{\circ} \mathrm{C}$ ) before decreasing back towards normal (hour 6- DL $39.51 \pm 0.26^{\circ} \mathrm{C}$, SL 39.32 $\pm 0.14^{\circ} \mathrm{C}$ ) (Figure 21a). Although, PBS did not change temperature, the PBS- treated Suffolk ewes had a 
lower temperature than PBS-treated Dorset ewes (DP $39.3 \pm 0.07^{\circ} \mathrm{C}$, SP: $38.8 \pm 0.03^{\circ} \mathrm{C}, \mathrm{p}=0.0073$ )

(Figure 21a).

The change in temperature at each hour from hour zero was affected by LPS (LPS $0.88 \pm 0.06^{\circ} \mathrm{C}$, PBS $\left.0.09 \pm 0.03^{\circ} \mathrm{C}, \mathrm{p}<0.0001\right)$, hour $(\mathrm{p}=0.0005)$, and the interactions LPS $x$ breed (DL $0.59 \pm 0.09^{\circ} \mathrm{C}, \mathrm{SL}$ : $\left.1.08 \pm 0.07^{\circ} \mathrm{C}, \mathrm{p}=0.0314\right)$ and LPS $\mathrm{x}$ hour $(\mathrm{p}=0.0001)$ with LPS causing an increase in temperature in both breeds; however, the Suffolk ewes had a greater increase in temperature in response to LPS at hour 2 (DL $\left.0.56 \pm 0.18^{\circ} \mathrm{C}, \mathrm{SL} 0.84 \pm 0.1^{\circ} \mathrm{C}, \mathrm{p}=0.0024\right)$, hour 3 (DL $0.73 \pm 0.25^{\circ} \mathrm{C} ; \mathrm{SL} 1.32 \pm 0.18^{\circ} \mathrm{C}, \mathrm{p}<0.0001$ ), hour $4\left(\mathrm{DL} 0.82 \pm 0.28^{\circ} \mathrm{C}, \mathrm{SL} 1.51 \pm 0.19^{\circ} \mathrm{C} \mathrm{p}<0.0001\right)$, hour $5\left(\mathrm{DL} 0.61 \pm 0.3^{\circ} \mathrm{C}\right.$, SL $1.08 \pm 0.21^{\circ} \mathrm{C}$, $\mathrm{p}=0.0011)$, and hour $6\left(\mathrm{DL} 0.43 \pm 0.25^{\circ} \mathrm{C}, \mathrm{SL} 0.73 \pm 0.18^{\circ} \mathrm{C}, \mathrm{p}=0.0191\right)$ (Figure $\left.21 \mathrm{~b}\right)$.

\section{Behavior changes/Mucosal Responses}

Due to the low frequency of each individual sickness symptom, all observed mucosal responses (nasal discharge, vaginal discharge, coughing, and diarrhea) and behavioral changes (inappetence, lethargic, lying down, and heavy breathing) were summed for each ewe over the 12 hours post treatment. LPS administration caused ewes to have observable physiological changes (LPS $4.37 \pm 0.71$, PBS $0.12 \pm$ $0.08, \mathrm{p}<0.0001)$ and Suffolk ewes administered LPS experienced a higher frequency of sickness symptoms than Dorset ewes (DL $1.18 \pm 0.62$, SL $6.56 \pm 0.72, \mathrm{p}=0.0029)$. The Dorset ewes that received PBS displayed $0.3 \pm 0.21$ symptoms while no symptoms were noted for any Suffolk ewes that received PBS (Figure 22).

Immune System

White Blood cell counts

Lipopolysaccharide (LPS 6,688,566 $\pm 241,538.4$, PBS 9,692,214 $\pm 200,412.5, p<0.0001$ ) treatment caused a decrease in WBCs in both breeds. There was also an effect of hour $(\mathrm{p}<0.0001)$ and an interaction of LPS $x$ hour ( $p<0.0001$ ). The LPS groups had significantly lower total WBCs at hour 1,2,3,4 (all $\mathrm{p}<0.0001$ ) and hour 5 ( $\mathrm{p}=0.0015)$. White blood cells decreased between hour 0 and hour 1 in both breeds receiving LPS. In the SL ewes, total WBCs stayed at that low level at hours 2 and 3 before gradually increasing. In the DL ewes, after the decrease in WBCs from hour 0 and 1 , cell numbers started gradually increasing (Figure 23).

\section{Acute Phase Proteins}

Plasma Hp was influenced by breed (Dorset $0.04 \pm 0.003 \mathrm{mg} / \mathrm{ml}$, Suffolk $0.12 \pm 0.01 \mathrm{mg} / \mathrm{ml}$, $\mathrm{p}=<0.0001)$, hour $(\mathrm{p}=0.0405)$, and the interaction of breed by LPS ( $\mathrm{p}=0.0357)$ with Suffolk ewes having more Hp than Dorset ewes receiving LPS (DL $0.05 \pm 0.01 \mathrm{mg} / \mathrm{ml}$, SL $0.10 \pm 0.01$ ). All groups had an increase in Hp levels from hour 12 to 24, but the largest increase was in the SL group (Figure 24a). Plasma SAA was affected by the interactions of breed $\mathrm{x}$ hour $(\mathrm{p}<0.0001)$ and breed $\mathrm{x}$ LPS $\mathrm{x}$ hour 
$(\mathrm{p}=0.0330)$, which was due to fluctuations in SAA in both breeds when treated with LPS over the sample time (Figure 24b).

\section{Cortisol}

Plasma cortisol increased in response to LPS (LPS $59.30 \pm 6.58 \mathrm{ng} / \mathrm{ml}$, PBS $30.93 \pm 3.25 \mathrm{ng} / \mathrm{ml}$, $\mathrm{p}=0.0029$ ). There were also effects of breed (Dorset $35.62 \pm 4.24 \mathrm{ng} / \mathrm{ml}$, Suffolk $54.61 \pm 6.31 \mathrm{ng} / \mathrm{ml}$, $\mathrm{p}=0.0284$ ) and the interactions breed $x$ LPS (DL $40.10 \pm 6.75 \mathrm{ng} / \mathrm{ml}, \mathrm{SL} 78.5 \pm 9.98 \mathrm{ng} / \mathrm{ml}, \mathrm{p}=0.0257$ ), LPS $x$ Hour $(\mathrm{p}<0.0001)$, and breed $x$ LPS $x$ hour $(\mathrm{p}=0.0085)$. Cortisol increased right after LPS administration in the Suffolk ewes and stayed elevated until hour 3 before decreasing back to normal by hour 12. In the Dorset ewes receiving LPS, cortisol did not increase until after hour 2, peaked at hour 3, and decreased back to normal by hour 6. Lipopolysaccharide had significant effects on cortisol at hour 1 ( $\mathrm{p}<0.0001)$, hour $2(\mathrm{p}=0.0006)$, hour $3(\mathrm{p}<0.0001)$, and hour $6(\mathrm{p}=0.0156)$. However, this was mainly due to the high values in the Suffolk ewes at those time points as the Dorset ewes had an increase in cortisol at only hour 3. (Figure 25).

\section{Gene Expression}

Lipopolysaccharide treatment upregulated CXCL8 expression (LPS $17.74 \pm 3.8$, PBS $2.26 \pm 0.44$, $\mathrm{p}=0.0003)$ similarily in both breeds. There was an hour $(\mathrm{p}=0.0005)$ and a LPS $\mathrm{x}$ hour $(\mathrm{p}=0.0142)$ interaction. CXCL8 increased from treatment administration (DL $3.67 \pm 1.93$, SL $6.54 \pm 2.87$ ) until hour 4 (DL $58.19 \pm 53.37$, SL $48.66 \pm 18.05$ ) before decreasing back to normal by hour 9 (DL $2.29 \pm 1.12$, SL $1.29 \pm 0.21$ ). The PBS groups had no change in CXCL8 expression (Figure 26a).

TLR4 expression was upregulated in response to LPS (LPS $3.78 \pm 0.55$, PBS $1.78 \pm 0.26$, $\mathrm{p}=0.0036$ ) and was also affected by breed (Dorset $3.58 \pm 0.52$, Suffolk $1.98 \pm 0.36, \mathrm{p}=0.0030$ ), hour $(\mathrm{p}=0.0121)$, and LPS $\mathrm{x}$ hour $(\mathrm{p}=0.0105)$. In the DL group, TLR4 expression increased from treatment administration $(1.93 \pm 0.77)$ to hour 3 when it peaked $(9.57 \pm 1.86)$, and then decreased back to normal by hour $9(1.88 \pm 0.21)$. In the SL group there was a more gradual increase in TLR4 expression from treatment administration until hour 6 (hour $00.96 \pm 0.25$, hour $65.38 \pm 1.49$ ) followed by a decrease until hour 9 (2.2 \pm 0.67$)$. While PBS did not cause a change in TLR4 expression, Dorset ewes that received PBS had greater TLR4 expression at hour 1.5 (DP $3.92 \pm 1.29$, SP $0.59 \pm 0.22, \mathrm{p}=0.0004$ ), and hour 3 (DP $3.40 \pm 0.86$, SP $0.71 \pm 0.26, \mathrm{p}=0.0023$ ) than Suffolk ewes (Figure 26d).

Nuclear factor-kappa B expression spiked at hour 6 in Suffolk ewes receiving LPS and PBS while there was no major changes in expression in the Dorset ewes. The breed x LPS interaction had a $p$ value of 0.0443 but using the Benjamini-Hochberg procedure, this was no longer significant. There was no 
effect of breed, LPS, hour, or any interaction effect on NF-kB expression (Table 10). There was no main effect or any interaction effects on TNFa expression (Table 10).

Interleukin-6 expression was affected by breed (Dorset $0.46 \pm 0.08$, Suffolk $3.82 \pm 0.52$, $\mathrm{p}=0.0007$ ) and breed $x$ LPS (DL $0.3 \pm 0.08$, SL $2.39 \pm 0.57, \mathrm{p}=0.0024)$ with the Suffolk ewes treated with LPS starting out with greater IL-6 expression and having an increase in IL-6 expression in response to LPS. The other 3 groups did not have a change in IL-6 expression. Compared to the SP group, the SL group had significantly greater IL-6 expression at hour 0 (SP $0.44 \pm 0.17$, SL 1.42 \pm 0.71 , p=0.0046), 15 minutes (SP $0.68 \pm 0.34$, SL $1.79 \pm 0.9, \mathrm{p}=0.018)$, 45 minutes (SP $0.42 \pm 0.21$, SL $2.46 \pm 1.95$, $\mathrm{p}=0.0122)$, hour 1.5 (SP $0.48 \pm 0.2$, SL $1.29 \pm 0.64$, p=0.0209), hour $2(\mathrm{SP} 0.48 \pm 0.18$, SL $4.18 \pm 3.22$, $\mathrm{p}=0.0196)$, hour 3 (SP $0.43 \pm 0.21$, SL $2.33 \pm 0.38,0.0137$ ), and hour 6 (SP $0.83 \pm 0.56$, SL $3.1 \pm 1.58$, $\mathrm{p}=0.0004)$ post-treatment (Figure 26b).

Expression of IFN $\gamma$ was affected by breed (Dorset $2.29 \pm 0.61$, Suffolk $2.99 \pm 0.59, \mathrm{p}=0.0153$ ) where the Suffolk ewes had higher expression compared to the Dorset ewes especially at hour 0 (Dorset $2.3 \pm 1.08$, Suffolk: $4.43 \pm 2.06$ ), hour 6 (Dorset: $1.02 \pm 0.45$; Suffolk: $2.68 \pm 3.45$ ) and hour 9 (Dorset $1.16 \pm 0.59$, Suffolk: $3.05 \pm 1.92$ ) (Figure 26c). COX 2 expression also was affected by breed (Dorset $0.43 \pm 0.05$, Suffolk: $1.43 \pm 0.19, \mathrm{p}=0.0002$ ) with Suffolk ewes having higher expression than Dorset ewes. The SL group had its highest COX2 expression at 45 minutes post treatment (DP $0.58 \pm 0.29$, DL $0.18 \pm 0.04$, SP $0.52 \pm 0.26$, SL $2.94 \pm 1.83$ ), and the SP group had its highest expression 30 minutes post treatment (DP 0.55 \pm 0.23 , DL $0.5 \pm 0.30$, SP $2.08 \pm 0.9$, SL $0.88 \pm 0.3)$. Expression in the DL $(0.32 \pm$ $0.07)$ group was different from expression in both the SL $(1.65 \pm 0.35)(\mathrm{p}=0.0022)$ and the SP $(1.2 \pm 0.16)$ $(\mathrm{p}=0.0040)$ group. The DP $(0.53 \pm 0.07)$ group was not different from any other group (Figure 26e).

Lipopolysaccharide treatment increased C3 expression (LPS $2.11 \pm 0.48$, PBS $0.85 \pm 0.25$, $\mathrm{p}=0.0004$ ). Suffolk ewes had higher C3 expression (Dorset $0.74 \pm 0.08$, Suffolk $2.22 \pm 0.53, \mathrm{p}=0.0077$ ), and expression was affected by hour $(\mathrm{p}<0.0001)$, and the interactions breed $x$ LPS (DL $0.85 \pm 0.16$, SL $3.32 \pm 0.87, \mathrm{p}=0.0004)$ and LPS $\mathrm{x}$ hour $(\mathrm{p}<0.0001)$. In the DL ewes, expression started increasing after hour 1 (DL 0.20 \pm 0.01 ). In response to LPS, the Suffolk ewes had a spike in C3 expression at 45 minutes (SL $3.10 \pm 2.1)$ and expression increased after hour $1(0.49 \pm 0.16)$. At hour 6, expression was still elevated in both LPS groups (DL $2.09 \pm 0.3$, SL $14.33 \pm 4.16$ ) (Figure 26f).

There was an effect of LPS (LPS $0.52 \pm 0.1$, PBS $1.20 \pm 0.14, \mathrm{p}=0.0028)$ and hour $(\mathrm{p}=0.0003)$ on MRC1 expression. The DP, SP, and SL groups had some fluctuation in expression over time but only the 
DL group had a pattern where expression decreased after LPS treatment until hour 9 and then stayed low (Figure 27a).

Interleukin-10 expression was affected by breed (Dorset $5.95 \pm 1.51$, Suffolk $2.93 \pm 1.10$, $\mathrm{p}=0.0448$ ) with the SL ewes having a spike in expression at hour 6 (DP $2.61 \pm 2.23$, DL $4.61 \pm 2.44$, SP $0.46 \pm 0.33$, SL $14.15 \pm 11.56$ ) and the DL ewes having an increase in expression from hour 6 to 12 with significantly higher values at hour 12 compared to the DP ewes (DP $1.58 \pm 0.68$, DL $23.54 \pm 13.28$, $\mathrm{p}=0.0049(\mathrm{SP} 0.56 \pm 0.4$, SL $0.13 \pm 0.063))($ Figure 27b).

Peroxisome proliferator-activated $\gamma$ expression was affected by breed (Dorset: $1.36 \pm 0.16$; Suffolk: $0.65 \pm 0.07, \mathrm{p}=0.0016$ ) with Dorset ewes having a higher fold change in PPAR $\gamma$ expression than Suffolk ewes. LPS (LPS: $0.78 \pm 0.1$; PBS: $1.24 \pm 0.15, \mathrm{p}=0.0056)$, hour $(\mathrm{p}=0.0059)$, and the interaction LPS $\mathrm{x}$ hour $(\mathrm{p}=0.0054)$ also affected PPAR $\gamma$ expression. In both breeds, LPS caused a spike in expression at hour 3 that then returned to normal levels. At hour 9, the SL group had a lower fold change in expression compared to the DL group (DL: 0.62 \pm 0.24 ; SL: $0.2 \pm 0.04 ; \mathrm{p}=0.0497$ ). The DP ewes had the highest fold change in PPAR $\gamma$ expression, being different from the SP ewes at hour 3 (DP: 1.80 \pm 0.86 ; SP: $0.51 \pm 0.16 ; p=0.0338$ ) and hour 6 (DP: 2.78 \pm 0.94 ; SP: 0.7 $\pm 0.18 ; p=0.0147$ ) (Figure 27e).

Forkhead box P 3 expression was affected by breed (Dorset $1.13 \pm 0.10$, Suffolk $0.54 \pm 0.05$, $\mathrm{p}=0.0001$ ) with Dorset ewes having greater fold change in expression than Suffolk ewes. The biggest differences occurred at hour 0 and hour 24. At hour 0 , the two LPS groups differed by breed (DL $1.48 \pm$ 0.85, SL $0.37 \pm 0.09, \mathrm{p}=0.0148$ ) as did the two PBS groups (DP $1.45 \pm 0.4$, SP $0.42 \pm 0.16, \mathrm{p}=0.0333$ ). The two LPS groups differed from each other at hour 24 (DL $1.08 \pm 0.62$, SL $0.29 \pm 0.1, \mathrm{p}=0.0382$ ) as did the two PBS groups (DP $1.34 \pm 0.13$, SP $0.51 \pm 0.10, \mathrm{p}=0.0203$ ). The interaction of LPS $\mathrm{x}$ hour $(\mathrm{p}<0.0001)$ affected FOXP3 expression. LPS caused a decrease in expression in the Dorset ewes from hour 3 to 6, which then rebounded by hour 24. In the Suffolk ewes, LPS caused an increase in expression from hour 0 to 2 after which expression decreased back to pre-treatment values (Figure 27f).

Transforming growth factor $\beta$ was affected by hour $(\mathrm{p}=0.0161)$ with most of the change in expression occurring in the Suffolk ewes. This breed had greater expression than the Dorset ewes at several time points, but the breed effect did not reach significance $(\mathrm{p}=0.0808)$. The Suffolk ewes had greater expression compared to the Dorset ewes at hour 6, however. Compared to the DP ewes, the SP ewes had more expression at hour 6 (DP $0.69 \pm 0.14$, SP $2.14 \pm 0.79, \mathrm{p}=0.0131$ ). Compared to the DL group, the SL group had higher expression at hour 6 (DL $0.69 \pm 0.14$, SL $2.72 \pm 1.35$, p=0.0006) (Figure 27c). 
Lipopolysaccharide (LPS $3.88 \pm 0.44$, PBS $1.49 \pm 0.15, \mathrm{p}=<0.0001)$ treatment, hour $(\mathrm{p}=0.0001)$, and their interaction (LPS $\mathrm{x}$ hour $\mathrm{p}<0.0001$ ) affected SOD2 expression. In the Dorset ewes, LPS caused a sharp increase in SOD2 expression from hour 0 until hour 3. Expression then decreased until hour 12 with a slight increase at hour 24. In the Suffolk ewes, LPS caused a more gradual increase in SOD2 expression until hour 6 followed by a gradual decrease until hour 24. LPS caused a greater increase in the Dorset compared to the Suffolk ewes, and the DL group had greater SOD2 expression compared to the SL group at hour 1.5 (DL 4.87 \pm 1.42 , SL $2.18 \pm 0.74, \mathrm{p}=0.0415$ ) and hour 3 (DL 10.82 \pm 2.07 , SL $4.62 \pm 1.28$, $\mathrm{p}=0.0292$ ). The DL group also had a higher fold change in expression than the SL group at hour 24 (DL $2.94 \pm 0.57$, SL $1.13 \pm 0.25, \mathrm{p}=0.0205$ ) due to expression in that group having a slight increase from hour 12 to 24 . Superoxide dismutase 2 expression was also affected by breed (Dorset $3.58 \pm 0.42$, Suffolk 1.81 $\pm 0.25, \mathrm{p}=0.0002$ ) and while the PBS groups had no major changes in expression, the DP group had greater expression compared to the SP group at hour 1.5 (DP $3.06 \pm 1.05$, SP $1.08 \pm 0.28, \mathrm{p}=0.0225$ ), hour 3 (DP $2.58 \pm 0.42$, SP $0.62 \pm 0.14, \mathrm{p}=0.0006)$, and hour 12 (DP $1.86 \pm 0.49$, SP $0.57 \pm 0.21, \mathrm{p}=0.0102$ )

(Figure 27d).

\section{Reproductive System}

Progesterone

Neither breed nor treatment affected plasma progesterone on day 8/9 post coitus $(3.38 \pm 0.18$ $\mathrm{ng} / \mathrm{ml})$ or on day $25 / 26$ post coitus $(3.93 \pm 0.15 \mathrm{ng} / \mathrm{ml})$ (Figure 28$)$.

\section{Pregnancy diagnosis/lambing data}

Pregnancy diagnosis was determined by ultrasound on day 25/26 and the number of ewes found to be pregnant was greatest in the SL group ( $\mathrm{p}=0.0421$ ) (Figure 29a) but there was no difference when only comparing between breeds (Dorset $42.86 \%$, Suffolk $68.75 \%, \mathrm{p}=0.0613$ ). After the ewes lambed, the date of lambing and the average gestation length was used to backtrack to day of conception. Neither experimental group $(p=0.0873$, Figure $29 b)$, breed $(p=0.0564)$, nor treatment $(p=0.5007)$ affected loss of pregnancy. Logistic regression confirmed the contingency analyses; neither treatment, breed, nor the interaction could predict the odds of a ewe having a lamb ( $\mathrm{p}=0.0987)$. The number of lambs born from each group from mating's that occurred during the experiment did not differ between the groups $(\mathrm{p}=0.3568$, data not shown).

\section{Discussion}

The temperature response to LPS in Suffolk ewes was about double compared to Dorset ewes. Although there were no differences in pregnany rates in this study, an elevation in body temperature associated with infection could affect embryonic loss as research studying heat stress in cattle that raised body temperature during embryonic development (Days 1 to 7 ) led to decreased embryonic survival 
(embryos recovered/assessed on day 7) (Putney et al., 1988). As the Suffolk ewes had a greater fever response, embryos of Suffolk ewes are likely to be at a greater risk of damage due to heat. Temperature is increased in response to IL-1 $\beta$ and IL-6, mediated by $\mathrm{PGE}_{2}$; SL ewes had a greater increase in IL- 6 gene expression compared to the DL ewes, which fits with them having a greater increase in temperature. IL$1 \beta$ and TNF $\alpha$ stimulate IL-6 production (and production of each other); SL ewes had high gene expression of IL-6; and IL- $1 \beta$ is a major driver of the fever response. $\mathrm{PGE}_{2}$ produced peripherally in response to pathogenic stimuli can travel through the blood-brain barrier and initiate a fever response (Netea et al., 2000; Evans et al., 2015). Suffolk ewes had a greater change in gene expression of COX2 and while specific PGs were not measured, LPS is known to stimulate production of $\mathrm{PGE}_{2}$ (Herath et al., 2009). Elevated peripheral $\mathrm{PGE}_{2}$ production would also contribute to the increased fever response in Suffolk ewes. Suffolk ewes tended to have a greater increase in expression of NF-kB which leads to PIC production which fits with the greater fever response. IL-1 $\beta$ causes $\mathrm{PGF}_{2 \alpha}$ and $\mathrm{PGE}_{2}$ secretion by endometrial stromal and epithelial cells (Davidson et al., 1995) while TNF $\alpha$ has been shown to cause $\mathrm{PGF}_{2 \alpha}$ secretion by stromal endometrial cells through activation of $\mathrm{PLA}_{2}$ (Skarzynski et al., 2000). Prostaglandin $\mathrm{F}_{2 \alpha}$ is not only luteolytic but is thought to have negative effects on embryo development and may be embryotoxic, especially during the morula to blastocyst transition (Buford et al., 1996; Seals et al., 1998; Scenna et al., 2004). If Suffolk ewes produce more $\mathrm{PGF}_{2 \alpha}$ during inflammation, they may be more susceptible to embryonic loss in response to bacterial infections during the pre-implantation period.

Acting on the brain, PICs cause behavioral changes that serve as an adaptive response to enhance recovery by conserving energy to fight acute inflammation. These behavioral changes include appetite disturbances, sleep disturbances, reduction of locomotor activity, exploration and grooming, reduced interest in the social and physical environment, loss of sexual interest, and impaired cognitive abilities (Konsman et al., 2002). Circulating LPS causes mucosal responses in the respiratory, digestive, and reproductive tracts. Observable changes in response to LPS that were noted during the experiment included lethargy, lying down, inappetence, heavy breathing, coughing, diarrhea, vaginal discharge, and nasal discharge. Ewes challenged with LPS had noticeable changes in sickness symptoms. The increase in discomfort and greater fever response in Suffolk ewes suggests a greater immune response to LPS in this breed.

The number of WBCs in the circulation decreased in response to LPS from hour 0 to 1 before slowly recovering over the next few hours. After treatment, the WBCs end up in the lungs and muscosal linings where they are responsible for the muscosal and respiratory changes. The change in WBCs in the LPS groups confirms that the LPS elicited an immune response. The first innate immune cells to be attracted to an inflammatory site are neutrophils, with one of their chemokines being CXCL8, activated 
by NF-кB. Interleukin-8 gene expression increased in response to LPS, confirming that an inflammatory response was generated. Nuclear factor- $\mathrm{KB}$ activation occurs in response to a signaling cascade initiated by a PAMP binding to a PRR; TLR4 is the PRR that binds LPS. Lipopolysaccharide treatment increased TLR4 expression, and Dorset ewes had more TLR4 expression, which potentially means there could be increased interaction between LPS and TLR4 in the Dorset ewes leading to a greater inflammatory response. Indeed, infertile cows had greater endometrial expression of TLR4, IL-1beta, and a greater ratio of IL-1 $\beta$ to IL-10 (Herath et al, 2009). However, looking at temperature response, symptoms, cortisol levels, and gene expression of other inflammatory mediators, the Dorset ewes had a reduced inflammatory response compared to the Suffolk ewes suggesting better clearance mechanisms. The peripheral blood mononuclear cells in Dorset ewes may be releasing TLR4 to its soluble form, which still binds LPS but does not increase cytokine production. Instead, soluble TLR4 binds LPS attached to LPSbinding protein, and the complex is them phagocytosed by macrophages (Oever et al., 2014). If this is occurring in the Dorset ewes it would explain the higher TLR4 gene expression compared to Suffolk ewes but the reduced inflammatory response to LPS.

The complement system is activated during bacterial infections to kill pathogens, promote inflammation through stimulating production of PICs, recruit inflammatory cells, and opsonize and clear immune complexes (Molina, 2005; Murphy, 2012). Proper control of complement in the uterine environment has been shown to be critical to prevent excessive inflammation and embryo loss (Xu et al., 2000; Mellor et al., 2001; Mao et al., 2003; Caucheteux et al., 2003). Expression of C3 in LPS challenged ewes increased with time starting from hour 1 but increased more in the Suffolk ewes. Immunoglobulin A will bind LPS in mucosal linings and cause activation of the classical complement pathway leading to production of pro-inflammatory cytokines. Suffolk ewes had increased C3 expression, and in response to LPS, an increased fever response, cortisol, sickness symptoms, and IL-6 expression. These responses are all downstream of pro-inflammatory cytokines that can be produced after complement activation.

The two major ovine APPs are Hp and SAA (Cray et al., 2009; Lecchi et al., 2012; Chapwanya et al., 2013) and are important in restoring homeostasis. Expression of SAA fluctuated over time, being affected by the interactions of breed $x$ hour and breed $x$ LPS $x$ hour. Lipopolysaccharide caused in increase in Dorset SAA but did not cause a change in Suffolk SAA perhaps putting Suffolk ewes at a disadvantage at resolving an infection/inflammatory response. Suffolk ewes had more Hp compared to Dorset ewes and had a larger increase in Hp in response to LPS. Hp has been reported to dampen LPS induced inflammatory effects in vitro and in vivo. Hp can bind to innate immune cells and in vitro reduced cytokine (TNF- $\alpha$, IL-12p70, IL-10) production by human PBMCs stimulated with LPS (Arredouani et al., 2005). 
Lipopolysaccharide led to activation of the HPA axis with a greater increase in corticol in the SL ewes. This could be a reflection of the greater IL-6 gene expression induced in these ewes by LPS and potentially greater $\mathrm{PGE}_{2}$ levels (increased COX2 expression). Cortisol is responsible to sickness symptoms and the increase in cortisol in the Suffolk ewes fits with their greater display of sickness symptoms. Downstream of cortisol and IL-6 is activation of APPs; therefore, greater concentrations of Hp levels in the Suffolk ewes also fits. Acute phase proteins are general markers of inflammation. Suffolk ewes having greater $\mathrm{Hp}$ might be a reflection of generally being in a more pro-inflammatory state reflected by having greater gene expression of COX2, C3, IFN $\gamma$, and lower gene expression of PPAR $\gamma$, Foxp3, and IL-10 (even without treatment).

There were no significant effects on NF-kB expression; however, the Suffolk ewes tended to have a greater increase in expression. The breed $x$ LPS effect was significant until the Benjamini-Hochberg procedure was used to control for multiple comparisons with the Suffolk ewes receiving LPS having a greater increase in NF-kB expression than Dorset ewes. Potentially more NF-kB in the Suffolk ewes would fit with the increase in IL-6 gene expression in this breed. NF-kB also causes transcription of TNF $\alpha$ and IL-1 $\beta$. While IL-1 $\beta$ was not measured, TNF $\alpha$ gene expression was not affected by breed or treatment. Gene expression does not always reflect protein levels, however, and TNF $\alpha$ protein has been shown to increase in response LPS in sheep (Graham, 2014) and has been shown to be embryotoxic (Soto et al., 2003a; Loureiro et al., 2007). Whether, there is a difference between Suffolk and Dorset ewes is yet to be determined. A difference in IFN $\gamma$ gene expression was shown with Suffolk ewes having higher expression. Because IFN $\gamma$ is embryotoxic by limiting trophectoderm proliferation (Hill et al., 1987), embryos in Suffolk ewes may be at a higher risk of damage compared to embryos in Dorset ewes.

PICs and ROS are damaging to embryos. IL-1 $\beta$ and TNF $\alpha$ stimulate the production of eicosanoids, NO, and ROS to help fight infections (Tracey, 2002), and nitric oxide has been shown to block development of preimplantation bovine embryos in vitro (Soto et al., 2003b). Although neither NO or ROS were measured, gene expression of SOD2, an enzyme that neutralizes ROS, increased with LPS treatment in both breeds. Neutrophils are a major producer of reactive oxygen species and are the first responders to any type of infection or inflammation. Increased expression of the neutrophil chemoattractant CXCL8 along with increased SOD2 expression indicates the elevated production of reactive oxygen species occurs in this LPS model. Expression of SOD2 increased more in the DL ewes, and greater expression would allow them to neutralize ROS more rapidly and minimize damage to other cells and the embryo.

Suffolk ewes had greater levels of IL-6, a cytokine with various functions depending on the presence and quantity of other mediators. It plays a role in regulating the balance between IL-17 
producing Th17 cells, which are pro-inflammatory and Treg cells that have suppressive functions. In conjunction with TGF $\beta$, IL-6 induces naïve CD4+ cells to polarize into Th17 cells. However, TGF $\beta$ in conjunction with IL-2 induces Treg polarization; IL-6 can inhibit this TGF $\beta$ function (Robertson et al., 2013). Not only is TGF $\beta$ important for polarization towards Treg cells, but it is produced by Tregs and tDCs to mediate some of their suppressive functions (Simpson et al., 2002; Ghiringhelli et al., 2005a).

Treg cells are not a terminally differentiated cell type; they can turn into Th1 or Th17 cells if exposed to high amounts of PICs, such as IL-6, IFN $\gamma$, or IL-1 (Gao et al., 2012). The only significant effect on TGF $\beta$ expression was hour, but Suffolk ewes tended to have a greater increase in expression of the cytokine. Expression in the SL ewes spiked at hour 6, the same hour the SL group had elevated expression of IL-6, and peak expression of TLR4, NF-אB, IFN $\gamma$, C3, IL-10, and SOD2. TGF $\beta$, IL-10, and SOD2 are anti-inflammatory mediators while the others are pro-inflammatory. With regards to inflammation at the maternal-fetal interface, the specialized immune cells in charge of regulating implantation and maternal tolerance to the fetus can change function. Tolerogenic DCs can become immunogenic, anti-inflammatory M2 MФs can become pro-inflammatory M1 MФs, uterine NKs can gain cytotoxic functions, and Tregs can become effector $\mathrm{T}$ cells. The necessity of maintaining these tolerogenic phenotypes has been demonstrated in various in vivo studies, and the balance of protein expression and activity of the pro- versus anti-inflammatory mediators will determine if immune cells change phenotype. Suffolk ewes having increased gene expression of IL-6 and TGF $\beta$ may mean their Tregs and switching to Th17 cells.

Treg cell depleted mice (Aluvihare et al., 2004; Zhao et al., 2007) and abortion-prone mice (Zenclussen et al., 2005) have demonstrated the necessity of Tregs in allogenic pregnancy to generate maternal tolerance to paternal antigen expressed by the fetus. Treg cell derived factors are needed by DCs for maintenance of their tolerogenic phenotype (Fiorentino et al., 1991b, Mahnke et al., 2007), by M2 MФs, and by uterine NKs cells to maintain their non-cytotoxic phenotype (Ghiringhelli et al., 2005a).

Wegorzewska et al. (2014) exerted a local inflammatory response by injecting LPS into the fetal liver of mice in utero and reported an increase in effector $\mathrm{T}$ cells $\left(\mathrm{CD} 8^{+} \mathrm{T}\right.$ cells and non-Treg $\mathrm{CD} 4^{+} \mathrm{T}$ cells) and a decrease in Treg cells in uterine tissue surrounding the resorbed fetuses (increased Teff/Treg ratio). An increase in CD25 ( $\alpha$ chain of the IL-2 receptor) on all of the T cell subsets may indicate increased activation of $\mathrm{T}$ cells in the uterus during inflammation and/or increased proliferation. Indeed, LPS induced inflammation in the fetus caused proliferation of both effector T cells and Treg cells with a higher proportion of effector $\mathrm{T}$ cells to Tregs in uterine tissue surrounding resorbed fetuses (Wegorzewska et al., 2014). A shift in the effector to Treg balance will result in embryonic loss due to 
loss of control over the tolerogenic uterine environment. Inflammatory mediators produced in response to infection enter systemic circulatation. The resident immune cells in the uterus during early pregnancy can be exposed to these circulating mediators and Suffolk ewes having a more intense inflammatory response may expose their uterine immune cells to elevated levels of these inflammatory mediators. This would increase the chance of the immune cells switching from a tolerogenic to immunogenic phenotype.

The transcription factor Foxp3 is the master regulator of multiple downstream pathways that govern Treg cell function. A decrease in Foxp3 could mean that there are less Treg cells or the cells are expressing less of the transcription factor, which will reduce their immune suppressive functions. Foxp3 expression was affected by LPS $\mathrm{x}$ hour causing decreases in Foxp3 expression. Suffolk ewes had significantly less Foxp3 expression compared to Dorset ewes suggesting Tregs in Suffolk ewes have reduced suppressive function. This is critical since infection induced dampening of Treg suppression at a time when sustained tolerance to fetal antigen is essential, such as the peri-implantation period (Shima et al., 2010), plays a crucial role in pregnancy loss (Rowe et al., 2012b).

Transforming growth factor $\beta$ is an important regulator of inflammation. One method of action is cross-talk with TLR4 signaling pathways leading to decreased NF- $\kappa \mathrm{B}$ activation and production of PIMs. TGF $\beta$ null mice, even in the absence of an inflammatory trigger, have increased gene expression of TLR4, TNF $\alpha$, IL-1 $\beta$, and inducible NOS that can be prevented by blocking NF- $\kappa$ B activation. While Suffolk ewes tended to have higher TGF $\beta$ expression and had lower TLR4 expression, they had higher NF-kB and IL-6 expression suggesting a strong inflammatory response even with the presence of the antiinflammatory cytokine.

Interleukin-10 is an anti-inflammatory cytokine that downregulates expression of PICs and stimulates and reinforces changes to both the innate and adaptive immune system such as inducing regulatory phenotypes in DCs, MФs, and T cells (Fiorentino et al., 1991a; Fiorentino et al., 1991b; Maynard and Weaver, 2008). Interleukin-10 is involved in generating Treg cells and is produced by Tregs to mediate some of their suppressive functions (Maynard and Weaver, 2008; Shevach, 2009; Huber et al., 2011). This cytokine has been shown to be critical during an inflammatory response to prevent fetal loss (Murphy et al., 1990; Robertson et al., 2007; Prins et al., 2015). Suffolk ewes had reduced IL-10 expression which probably contributes to their enhanced inflammatory response compared to Dorset ewes. In addition, the lower IL-10 expression in Suffolk ewes may make their uterine immune cells more susceptible to phenotype switching during inflammation, which would be detrimental to pregnancy.

Interleukin-10 and TGF $\beta$ are produced by Tregs and tDCs in the uterus to regulate fetal tolerance and the cytokine milieu at the maternal-fetal interface (Simpson et al., 2002) regulating the 
response/phenotype of uNK cells to pathogens. While uNK cells do not display the cytotoxic functions of their peripheral counterparts, uncontrolled or exaggerated inflammation at the maternal-fetal interface can cause uNK cells to revert back into their peripheral NK cell phenotype and cause embryonic loss (Murphy et al., 2005; Murphy et al., 2009; Kwak-Kim et al., 2014). Uterine NK cells with cytotoxic capability cause apoptosis of trophoblast cells by transferring granulysin with the help of perforin (Nakashima et al., 2008). IL-12 stimulates IFNץ production by NK cells and induces their cytotoxic activity. The PICs TNF $\alpha$, IL-1 $\beta$, and IL-15 synergize with IL-12 to induce IFN $\gamma$ production and IL-15 also promotes NK cell maturation. IL-10 can regulate IFN $\gamma$ production by NK cells by inhibiting IL-12 production while TGF $\beta$ can block proliferation and cytotoxicity of NK cells as well as inhibit IFNץ and IL-12 production (Biron et al., 1999; Eriksson et al., 2004; Eriksson et al., 2006). While Suffolk ewes tended to have higher TGF $\beta$ gene expression, they had lower IL-10 gene expression and higher IFN $\gamma$ gene expression, which would make their uNKs vulnerable to becoming cytotoxic and killing the embryo.

Peroxisome proliferator-activator receptors (PPARs) are nuclear receptors and ligand activated transcription factors (Dinarello, 2010; Lian et al., 2015) with anti-inflammatory functions (Clark et al., 2000; Feige et al., 2006; Dinarello, 2010; Li et al., 2014; Lian et al., 2015) that are expressed in the ovine endometrium (Cammas et al., 2006). Peroxisome proliferator-activator receptor agonists reduce expression of several PICs, chemokines, and adhesion molecules and affect $\mathrm{T}$ cell proliferation (including in the placenta) by interfering and inhibiting other transcription factors (Ricote et al., 1998; Clark et al., 2000; Lappas et al., 2002; Genolet et al., 2004; Feige et al., 2006; Szanto and Nagy, 2008; Dinarello, 2010; Li et al., 2014; Lian et al., 2015). Lipopolysaccharide caused a decrease in PPAR $\gamma$ gene expression in both breeds after hour 3 post treatment. Dorset ewes however, had more PPAR $\gamma$ expression compared to Suffolk ewes. This also indicates Suffolk ewes will have a prolonged inflammatory response as methods to stop the production of inflammatory mediators are dampened in this breed.

Peroxisome proliferator-activator receptors $\gamma$ can inhibit gene expression and release of IL-6, COX-2, INFy and APPs while increasing IL-10 production (Ricote et al., 1998; Jiang et al., 1998; Jackson et al., 1999; Takano et al., 2000; Wang et al., 2001; Genoler et al., 2004; Bensinger and Tontonoz, 2008; Szanto and Nagy, 2008). The smaller change in PPARY in Suffolk ewes may be associated with the larger change in IL-6, COX2, IFN $\gamma$ and SAA and smaller change in IL-10 gene expression compared to Dorset ewes. PPAR $\gamma$ regulates the response of immune cells to inflammatory stimuli and influences the polarization of Th1 versus Th2 cells (Genolet et al., 2004). The transcription factor promotes alternative activation of MФs (M2) by preventing MФs from being activated in response to PIMs (M1 MФs) (Genoler et al., 2004; Bensinger and Tontonoz, 2008) and inhibits their response to LPS or IFN $\gamma$ (Ricote 
et al., 1998). In DCs, activation of PPAR $\gamma$ reduces the cell's ability to be stimulated by TLR agonists, its capacity to stimulate $\mathrm{T}$ cell proliferation, and inhibits cytokines for Th1 polarization and chemokines for Th1 cell recruitment (Faveeuw et al., 2000; Gosset et al., 2001; Appel et al., 2005). Lower PPARY activity in Suffolk ewes would lead to a more robust inflammatory response and, at the maternal-fetal interface, a change in the phenotypes of immune cells followed by embryonic loss.

Mannose receptor C type 1 is expressed by MФs and DCs to neutralize/clear pathogens and for antigen uptake and presentation (Shepard et al., 1990; Engering et al., 1997). The receptor recognizes complex carbohydrate structures on glycoproteins and mediates the endocytosis of glycoproteins by MФs. Lipopolysaccharide has been shown to decrease MRC1 activity on MФs in vitro that could be prevented by pre-treatment of the MФs with dexamethasone (Shepherd et al., 1990) likely due to GCs increasing mannose receptor synthesis (Shepherd et al., 1985). Lipopolysaccharide challenge in ewes caused a decrease in MRC1 expression with the most change occurring in the DL group; however, there was no breed x LPS effect. MRC1 expression may not have changed as much in the Suffolk ewes due to higher cortisol levels in this breed (Shepherd et al., 1985; 1990). Potentially reduced expression of the receptor by MФs and DCs during a bacterial infection would prolong clearance of the pathogen and increase the risk of embryonic loss.

Progesterone is a critical hormone for pregnancy (Bazer et al., 2008). There was no difference in P4 levels between the four groups on day 8/9 or 25/26 post coitus suggesting LPS challenge did not result in luteolysis, or ewes that were open had recycled and were back in a luteal phase. Pregnancy diagnosis by ultrasound had significantly more ewes in the SL group being pregnant compared to the other 3 groups. However, after lambing when conception date was determined retrospectively, there was no difference between groups on the percentage of ewes that carried a lamb(s) from the mating that occurred during the experiment (no difference in embryonic loss). Bacterial infections are known to decrease pregnancy rates, but this was not seen with the LPS challenge. This could be because the immune response to the experimental LPS challenge is less than 12 hours in duration for most of the factors measured, whereas, depending on the condition of the animal, an actual infection could last days to weeks. So while this LPS model provides insight into the mechanisms of bacterial infection induced embryonic loss, it may not sustain physiological changes long enough to cause a significant amount of embryonic loss.

Small sample size potentially limited detecting differences in embryonic loss. Suffolks have greater losses in embryos and fetuses compared to Dorset ewes (Dixon et al., 2014). As seen from the present results, Suffolk ewes tend to have a stronger inflammatory response that could result in a change 
in the production of hormones needed to time the "window of implantation" (including P4), could damage or cause death of the embryo, and/or change the phenotype of the local immune cells resulting in loss of maternal-fetal tolerance. Therefore, the difference in the inflammatory response seen between the two breeds in the present study may help explain the differences seen in pregnancy loss reported by Dixon et al. (2014). Both breeds originated in England and gram negative bacteria are everywhere; therefore, the differential inflammatory response between the breeds in unlikely to be due to selection pressure. Instead, perhaps different haplotypes in TLR4 exist that make the LPS response in the Suffolk ewes more severe (Koets et al., 2010). In using this model to mimic mastitis in the dairy cow, it is important to note that the breeds differ in their responses. This may impact the results of a study examining ways to dampen the inflammatory response to prevent embryonic loss; a given treatment may work better or worse in the Suffolk breed that experiences an elevated inflammatory response or the treatment dose may need to be adjusted. In addition, the response in one of the breeds may more closely match the response of a mastitic dairy cow and would provide more applicable results. 


\section{Tables}

Table 10: Fold change in nuclear factor kappa B and tumor necrosis alpha gene expression to LPS or PBS in early pregnant Dorset and Suffolk ewes. Data expressed as the fold change in gene expression using the $2^{-\Delta \Delta C t}$ method with GAPDH as the housekeeping gene and the reference time point being the sample collected at CIDR withdraw. Data analyzed by repeated measures ANOVA. Mean \pm SEM. Mean is the overall mean of the fold change across the time points but analyzed with time as the repeated measure. (SL $(n=16)$ : Suffolk ewe + LPS; SP ( $n=16)$ : Suffolk ewe + PBS; DL $(n=11)$ : Dorset ewe + LPS, DP $(n=10)$ : Dorset ewe + PBS)

\begin{tabular}{|c|c|c|c|c|c|c|c|c|c|}
\hline $\begin{array}{l}\text { Gene/Time } \\
\text { points }\end{array}$ & $\begin{array}{l}\text { Treat } \\
\text { ment } \\
\text { Group }\end{array}$ & $\begin{array}{l}\text { Mean } \pm \\
\text { SEM }\end{array}$ & $\begin{array}{l}\text { Breed } \\
\text { Effect }\end{array}$ & $\begin{array}{l}\text { LPS } \\
\text { Effect }\end{array}$ & $\begin{array}{l}\text { Breed x } \\
\text { LPS } \\
\text { Effect }\end{array}$ & $\begin{array}{l}\text { Hour } \\
\text { Effect }\end{array}$ & $\begin{array}{l}\text { Breed x } \\
\text { Hour }\end{array}$ & $\begin{array}{l}\text { LPS x } \\
\text { Hour }\end{array}$ & $\begin{array}{l}\text { Breed x } \\
\text { LPS x } \\
\text { Hour }\end{array}$ \\
\hline NF-кB & & & $\mathrm{p}=0.05$ & $\mathrm{p}=0.97$ & $\mathrm{p}=0.04$ & $\mathrm{p}=0.43$ & $\mathrm{p}=0.60$ & $\mathrm{p}=0.18$ & $\mathrm{p}=0.18$ \\
\hline \multirow{4}{*}{$\begin{array}{l}0,1.5,3,6 \\
9 \mathrm{hrs}\end{array}$} & SL & $2.62 \pm 0.72$ & & & & & & & \\
\hline & SP & $2.48 \pm 1.52$ & & & & & & & \\
\hline & DL & $0.58 \pm 0.14$ & & & & & & & \\
\hline & DP & $1.45 \pm 0.53$ & & & & & & & \\
\hline TNFa & & & $\mathrm{p}=0.41$ & $\mathrm{p}=0.06$ & $\mathrm{p}=0.67$ & $\mathrm{p}=0.24$ & $p=0.66$ & $\mathrm{p}=0.21$ & $\mathrm{p}=0.14$ \\
\hline \multirow[t]{4}{*}{$0,0.5,1 \mathrm{hr}$} & $\mathrm{SL}$ & $0.79 \pm 0.2$ & & & & & & & \\
\hline & SP & $0.49 \pm 0.13$ & & & & & & & \\
\hline & $\mathrm{DL}$ & $0.78 \pm 0.14$ & & & & & & & \\
\hline & $\mathrm{DP}$ & $0.53 \pm 0.09$ & & & & & & & \\
\hline
\end{tabular}

Figures
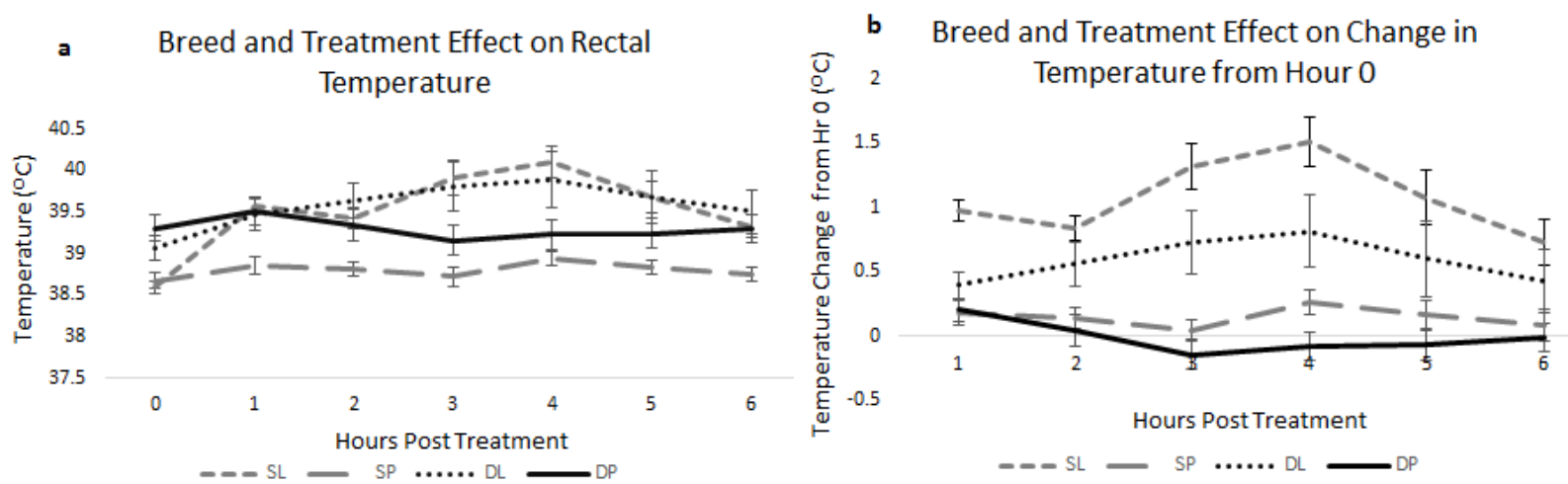

Figure 21: Response in rectal temperature to LPS or PBS in early pregnant Dorset and Suffolk ewes. (a) Hourly temperature: Breed p=0.0284, LPS p=0.0001, Hour p<0.0001, Breed $x$ Hour p=0.0330, LPS x Hour p<0.0001 (b) Change in rectal temperature at hours 1-6 compared to time 0: LPS $\mathbf{p}<\mathbf{0 . 0 0 0 1}$, LPS $\times$ Breed p=0.0314, Hour p=0.0005, LPS x Hour p=0.0001 (SL ( $n=16)$ : Suffolk ewe + LPS, SP ( $n=16)$ : Suffolk ewe + PBS, DL ( $n=11)$ : Dorset ewe + LPS, DP $(n=10)$ : Dorset ewe + PBS $)$. Data were analyzed by repeated measures ANOVA Mean \pm SEM 


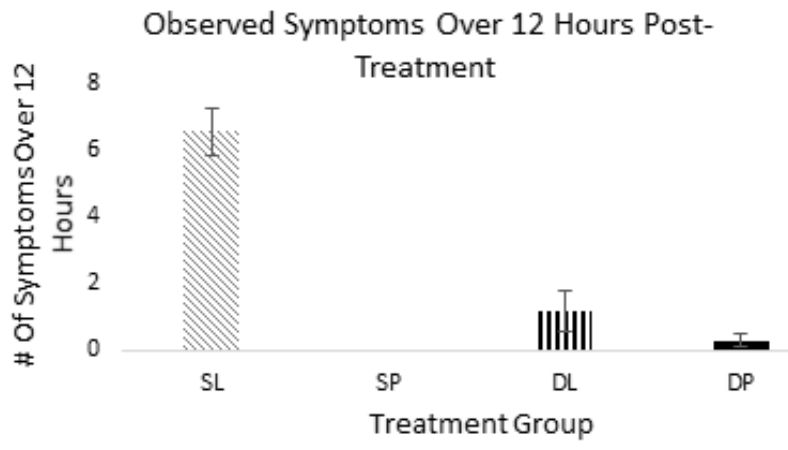

Figure 22: Frequency of sickness symptoms displayed by Suffolk and Dorset ewes receiving either LPS or PBS. Changes in behavior or noticeable sickness were noted at whole hour (hrs $0,1,2,3,4,5,6,9,12$ ) blood sample collection times, added up for each ewe over the 12 hours post-treatment, and averaged for each treatment group. LPS p<0.0001, LPS x Breed $\mathbf{p}=\mathbf{0 . 0 0 2 9}$ (SL $(n=16)$ : Suffolk ewe + LPS, SP $(n=16)$ : Suffolk ewe + PBS, DL ( $n=11)$ : Dorset ewe + LPS, DP $(n=10)$ : Dorset ewe + PBS) Data were analyzed by ANOVA. Mean \pm SEM

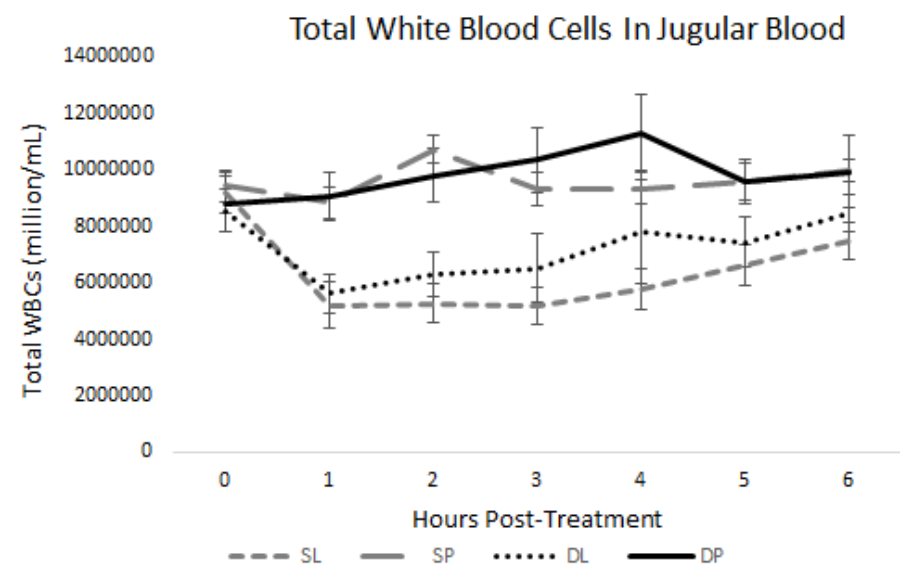

Figure 23: Response in white blood cells to LPS or PBS in early pregnant Dorset and Suffolk ewes from hours 0-6 post treatment. LPS $\mathbf{p}<\mathbf{0 . 0 0 0 1}$, Hour $\mathbf{p}<\mathbf{0 . 0 0 0 1}$, LPS x Hour $\mathbf{p}<\mathbf{0 . 0 0 0 1}$ ( $\mathrm{SL}(\mathrm{n}=16)$ : Suffolk ewe + LPS, SP ( $\mathrm{n}=16)$ : Suffolk ewe + PBS, DL $(n=11)$ : Dorset ewe + LPS, DP $(n=10)$ : Dorset ewe + PBS). Data were analyzed by repeated measures ANOVA. Mean \pm SEM 

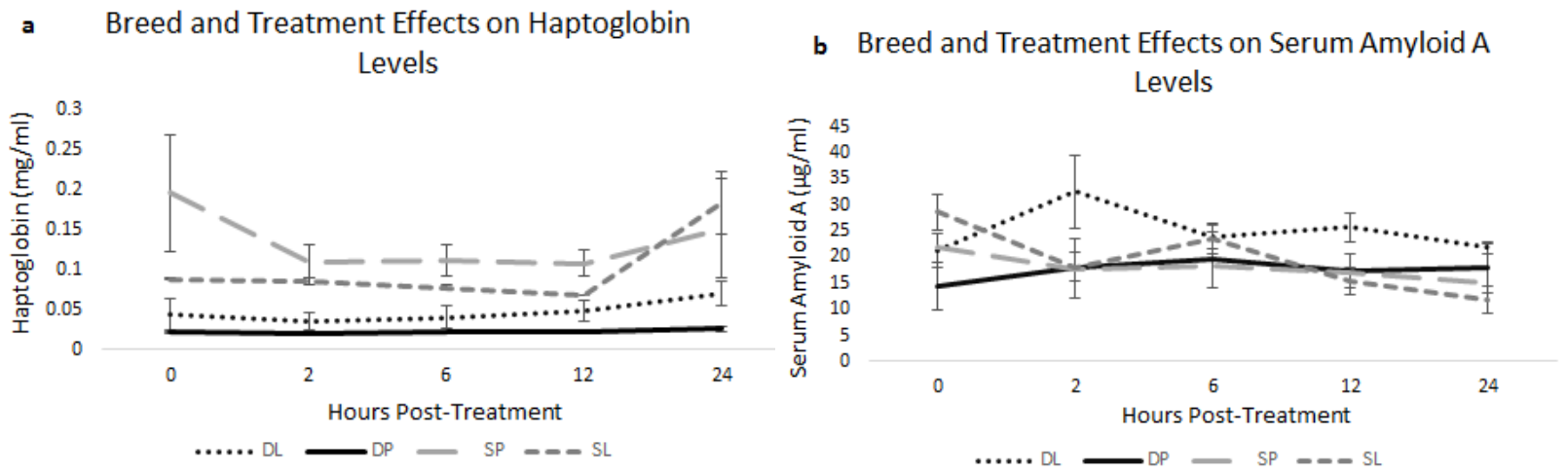

Figure 24: Response in acute phase proteins to LPS or PBS in early pregnant Suffolk and Dorset ewes from 0-24 hours post treatment. (a) Haptoglobin: Breed $\mathbf{p}<\mathbf{0 . 0 0 0 1}$, Breed $x$ LPS $\mathbf{p}=\mathbf{0 . 0 3 5 7}$, Hour $\mathbf{p}=\mathbf{0 . 0 4 0 5}$ (b) Serum Amyloid A: Breed x Hour p<0.0001, Breed x LPS x Hour p=0.0330 (SL ( $n=16)$ : Suffolk ewe + LPS, SP ( $n=16$ ): Suffolk ewe + PBS, DL $(n=11)$ : Dorset ewe + LPS, DP $(n=10)$ : Dorset ewe + PBS). Data were analyzed by repeated measures ANOVA. Mean \pm SEM

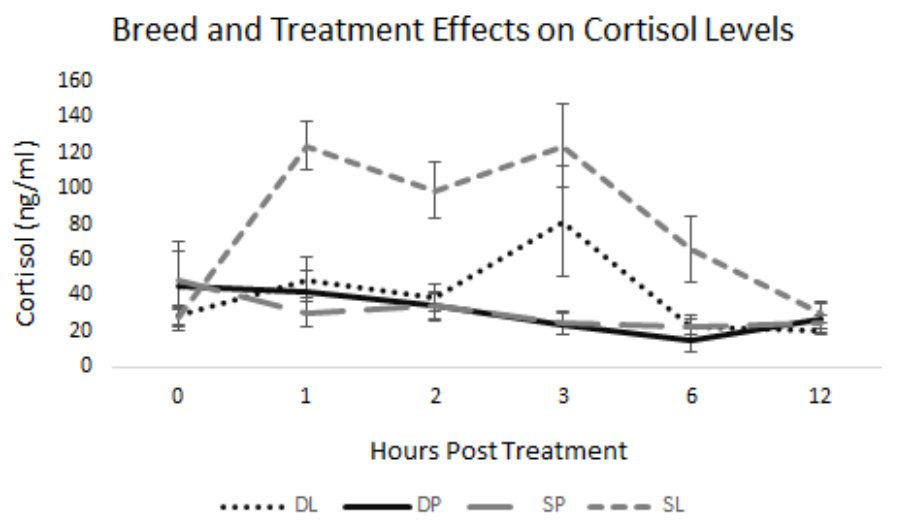

Figure 25: Cortisol response to LPS or PBS in early pregnant Dorset and Suffolk ewes from 0-12 hours post treatment. Breed $p=0.0284$, LPS $p=0.0029$, Breed $x$ LPS $p=0.0257$, Hour $p<0.0001$, LPS $x$ Hour $p<0.0001$, Breed $\mathbf{x}$ LPS $\mathbf{x}$ Hour $\mathbf{p = 0 . 0 0 8 5}$ (SL ( $\mathrm{n}=16)$ : Suffolk ewe + LPS, SP ( $\mathrm{n}=16)$ : Suffolk ewe + PBS, DL ( $\mathrm{n}=11)$ : Dorset ewe + LPS, DP $(n=10)$ : Dorset ewe + PBS). Data were analyzed by repeated measures ANOVA. Mean \pm SEM 

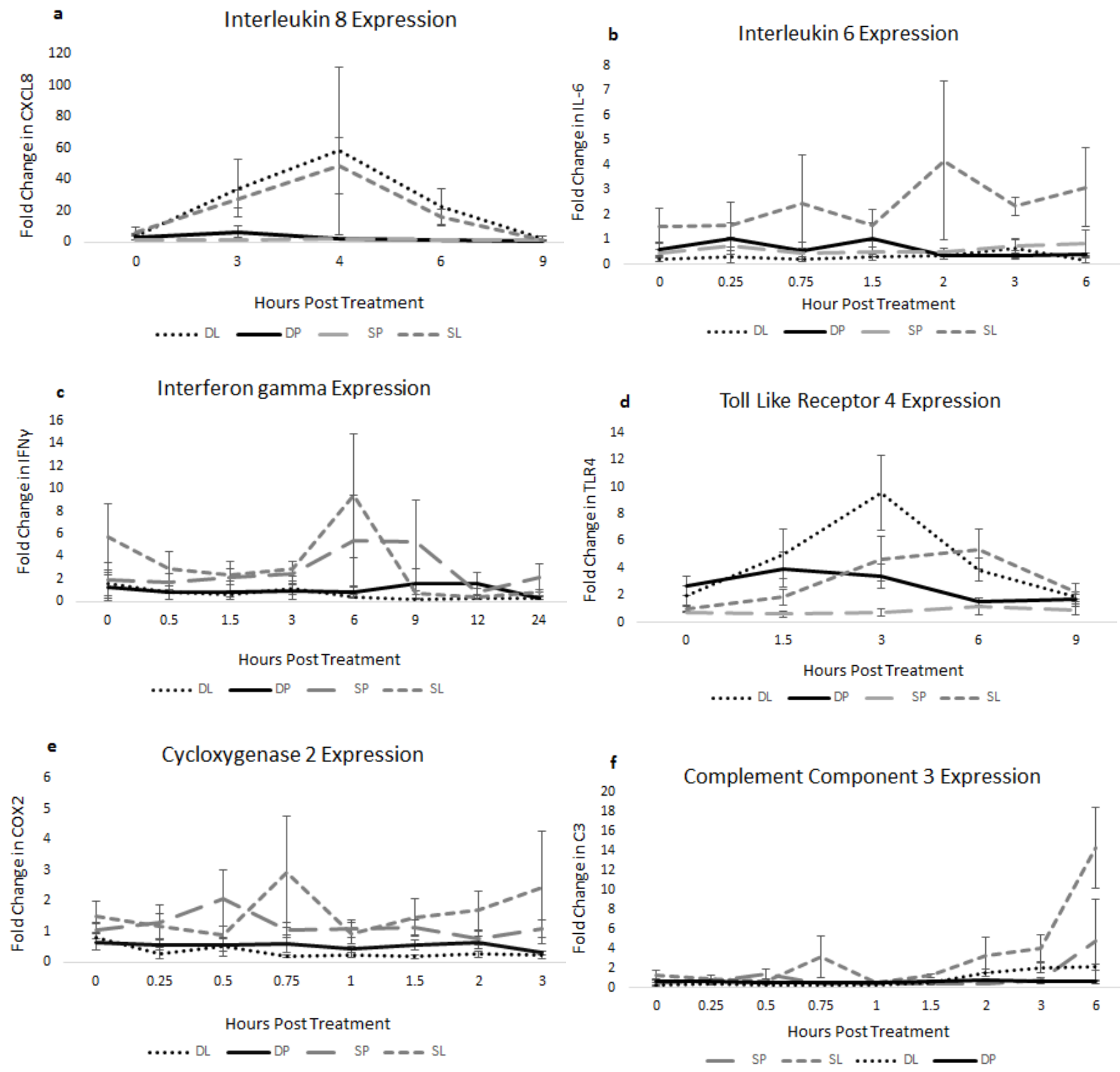

Figure 26: Fold change in gene expression of pro-inflammatory factors measured in white blood cells in response to LPS or PBS in early pregnant Suffolk and Dorset ewes. Gene Expression normalized to GAPDH and referenced to expression levels in the sample collected at CIDR withdraw. (a) CXCL8: LPS $\mathbf{p = 0 . 0 0 0 3 , ~ H o u r ~} \mathbf{p = 0 . 0 0 0 5}, \mathbf{L P S} \mathbf{x}$ Hour $\mathbf{p}=\mathbf{0 . 0 1 4 2}$ (b) IL-6: Breed $\mathbf{p}=\mathbf{0 . 0 0 0 7}$, Breed $\mathbf{x}$ LPS $\mathbf{p}=\mathbf{0 . 0 0 2 4}$ (c) IFN $\gamma$ : Breed $\mathbf{p}=\mathbf{0 . 0 1 5 3}$ (d) TLR4: Breed $p=0.0030$, LPS $p=0.0036$, Hour $p=0.0121$, LPS $x$ Hour $p=0.0105$ (e) COX2: Breed $p=0.0002$ (f) C3: Breed $p=0.0077$, LPS $p=0.0028$, Breed $x$ LPS $p=0.0004$, Hour $p<0.0001$, LPS $x$ Hour $p<0.0001$ (SL ( $n=16)$ : Suffolk ewe + LPS, SP $(n=16)$ : Suffolk ewe + PBS, DL $(n=11)$ : Dorset ewe + LPS, DP (n=10): Dorset ewe + PBS) Data were analyzed by repeated measures. ANOVA Mean \pm SEM 

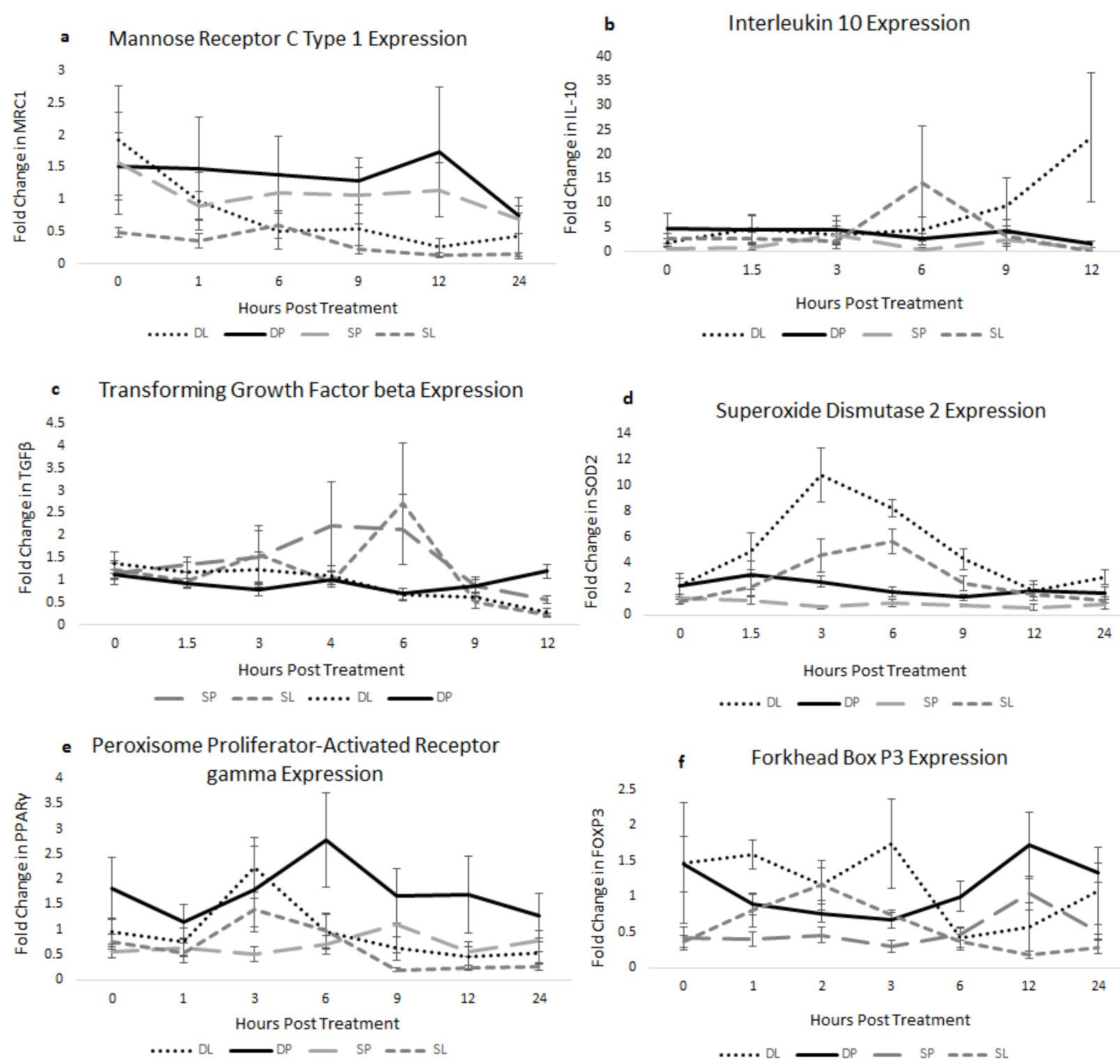

Figure 27: Fold change in gene expression of anti-inflammatory factors measured in white blood cells in response to LPS or PBS in early pregnant Suffolk and Dorset ewes. Gene Expression normalized to GAPDH and referenced to expression levels in the sample collected at CIDR withdraw. (a) MRC1: LPS $\mathbf{p = 0 . 0 0 2 8 , ~ H o u r ~} \mathbf{p}=\mathbf{0 . 0 0 0 3}$ (b) IL10: Breed $\mathbf{p}=\mathbf{0 . 0 4 4 8}$ (c) TGFß: Breed $p=0.0808$, Hour $\mathbf{p}=\mathbf{0 . 0 1 6 1}$ (d) SOD2: Breed $\mathbf{p}=\mathbf{0 . 0 0 0 2 , ~ L P S ~} \mathbf{p}<\mathbf{0 . 0 0 0 1}$, Hour $p=0.0001$, LPS $x$ Hour $p<0.0001$ (e) PPARy: Breed $p=0.0016$, LPS $p=0.0056$, Hour $p=0.0059$, LPS $x$ Hour p=0.0054 (f) Foxp3: Breed p=0.0001, LPS x Hour p<0.0001 (SL $(n=16)$ : Suffolk ewe + LPS, SP ( $\mathrm{n}=16)$ : Suffolk ewe + PBS, DL ( $n=11)$ : Dorset ewe + LPS, DP $(n=10)$ : Dorset ewe + PBS). Data were analyzed by repeated measures ANOVA. Mean \pm SEM 


\section{Progesterone Concentrations 25/26 Days Post}

\section{Coitus}

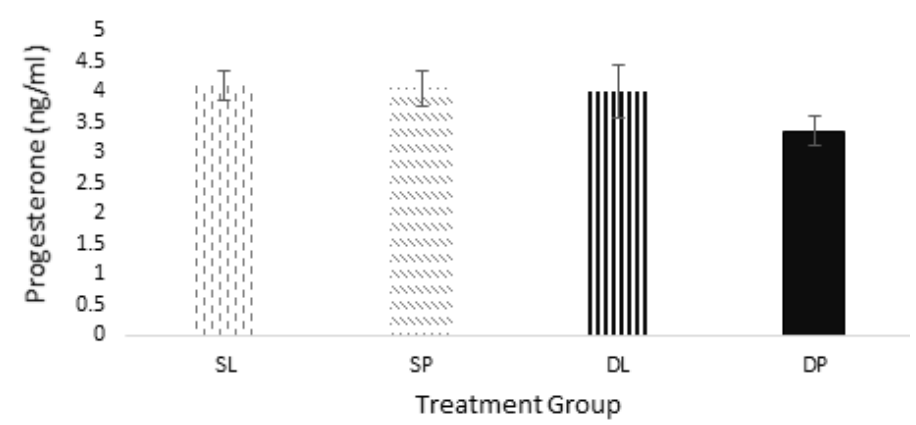

Figure 28: Plasma concentration of progesterone on day 25/26 post coitus in Dorset and Suffolk ewes administered LPS or PBS on day $5 / 6$ post coitus. Breed $p=0.2137$ LPS $p=0.2739$ Breed $x$ LPS $p=0.3254$. Data were analyzed by 2-way ANOVA. Mean \pm SEM (SL ( $n=16)$ : Suffolk ewe + LPS, SP ( $n=16)$ : Suffolk ewe + PBS, DL ( $n=11)$ : Dorset ewe + LPS, DP $(n=10)$ : Dorset ewe + PBS)
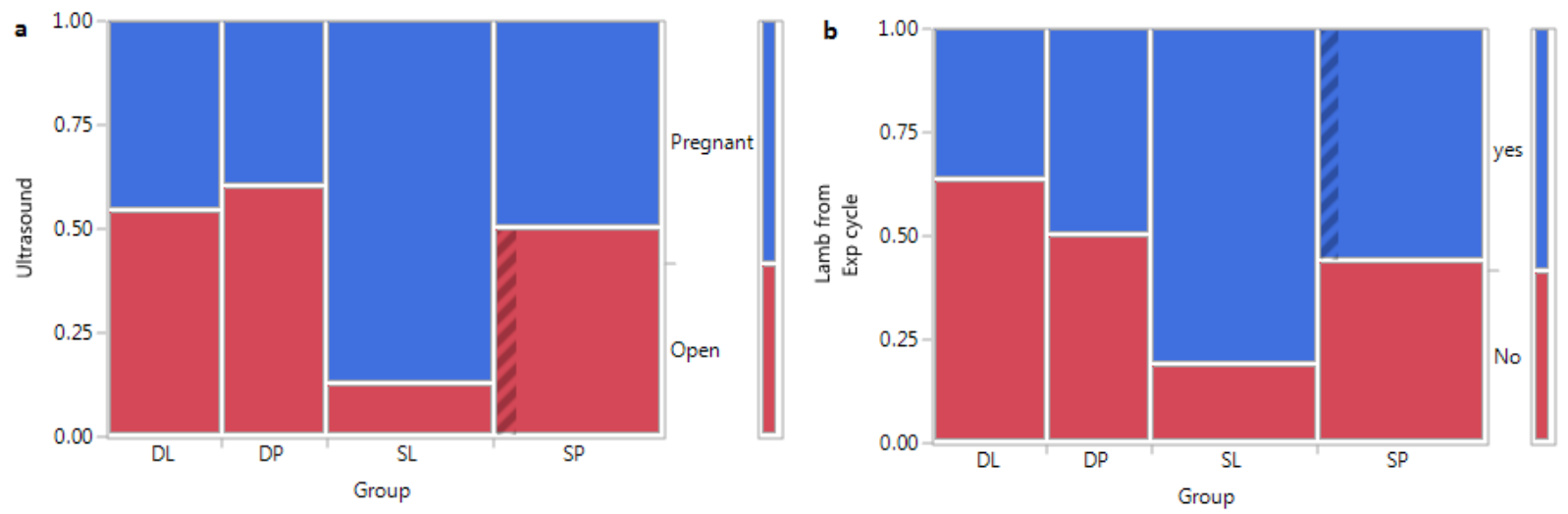

Figure 29: Analysis examining (a) whether a ewe was found to be pregnant or open by ultrasound on 25/26 days post coitus (Pearson's chi square $\mathbf{p}=\mathbf{0 . 0 4 2 1}$ ) or $(\mathbf{b})$ whether or not a ewe had a lamb as a result of the mating that took place during the experiment (Pearson's chi square $\mathrm{p}=0.0873$ ) was contingent on experimental group. (SL $(n=16)$ : Suffolk ewe + LPS, SP ( $n=16)$ : Suffolk ewe + PBS, DL $(n=11)$ : Dorset ewe + LPS, DP $(n=10)$ : Dorset ewe + PBS) 


\section{Chapter 5: Discussion}

Mastitis impacts the profit of dairy producers associated with lost milk production, clinical treatment, culling, and increased replacement animals. Bar et al. (2008), using information from five dairy farms in New York, estimated, that lost revenue from a case of clinical mastitis at \$179; milk loss (\$115), increased mortality $(\$ 14)$, and treatment associated costs $(\$ 50)$. However, as the price of milk increases, cost due to mastitis increases due to increased profit losses. Mastitis is often caused by environmental bacteria, such as gram negative $E$. coli, which induce inflammation via its cell wall component LPS. The energy demands of lactation early postpartum put dairy cows into a state of negative energy balance that impairs immune function, making them susceptible to infections. Dairy cows with mastitis, as well as other diseases, can experience various reproductive issues including impaired resumption of ovulation postpartum, compromised fertilization, impaired conceptus development, changes in expression of genes associated with conceptus development and implantation, increased early pregnancy loss, and reduced pregnancy per insemination. Overall, these reproductive issues increase the calving interval, which is detrimental to profits of dairy producers. Mechanisms of how periparturient diseases lead to reproductive issues has not been clarified (Santos and Ribeiro, 2014), but pre-implantation is known to be a critical period for embryonic loss in lactating dairy cows (Wiltbank et al., 2016).

Binding of LPS to TLR4, initiates an immediate response involving activation of intracellular signaling cascades and the expression of inflammatory mediators including IL-1 $\beta$, TNF $\alpha$, IL- 6 , IFN $\gamma$, CXCL8, and PGs. During mastitis, circulating levels of TNF $\alpha$, IL-1, and IL-6 can increase. Cells from infected mammary glands have increased mRNA expression for IL-1 $\beta$, TNF $\alpha$, IL-10, and IL-12 and increased TNF $\alpha$ protein (Riollet et al., 2001; Ślebodziński et al., 2002; Hansen et al., 2004). The inflammatory mediators have local and systemic effects and recruit additional immune cells to the infection site to eliminate the pathogen. These mediators can travel from the mammary gland to the reproductive tract and influence follicle growth, oocyte quality, uterine function, embryo development, and implantation (Turner et al., 2012).

Systemic administration of LPS to early pregnant ewes in the current studies consistently resulted in an inflammatory response characterized by fever, increased mucosal secretions, and behavioral changes, decreased number of circulating WBCs, increased serum Hp, activation of the HPA axis, increased gene expression of C3, CXCL8, TLR4, and SOD2, and decreased gene expression of MRC1, Foxp3, and PPAR $\gamma$. All of these changes were expected as the animal believes it has a bacterial infection that needs to be cleared.

Lipopolysaccharide signals through this TLR4 and LPS will activate the complement system. Downstream of TLR4 and complement (C3) is transcription of CXCL8 that recruits neutrophils to the 
inflammatory site. As neutrophils are the first responders to pathogen invasion, increased expression of CXCL8 and neutrophil mobilization were expected. Neutrophils use ROS to control the increase in, and spread of bacteria, but ROS are not specific when it comes to causing damage and can have effects on host cells. Therefore, antioxidants are secreted to convert ROS into less harmful molecules. In all three studies, gene expression of the antioxidant, SOD2, was upregulated with LPS treatment, suggesting an increase in ROS. Excessive ROS in the reproductive tract will damage embryonic and uterine cells. This might be one mechanism contributing to embryonic loss caused by bacterial infections.

Lipopolysaccharide, TNF $\alpha$, and IFN $\gamma$ have been shown to induce NO synthase and NO inhibits embryonic development (Athanassakis et al., 2000; Soto et al., 2003b). Thus, providing additional antioxidants during early pregnancy might benefit reproductive success. Suffolk ewes had a reduced change in expression of SOD2 compared to Dorset ewes, which may put their embryos at increased risk of oxidative damage. Therefore, therapies to reduce ROS may have siginificant effects on embryonic loss in Suffolk ewes more than Dorset ewes.

Lipopolysaccharide treatment consistently decreased PPAR $\gamma$, Foxp3, and MRC1 expression. Peroxisome proliferator-activated receptor $\gamma$ is a transcription factor important for inhibiting gene transcription of pro-inflammatory mediators such as IL-6, COX-2, INF $\gamma$, and APPs while increasing expression of anti-inflammatory IL-10 (Ricote et al., 1998; Jiang et al., 1998, Jackson et al., 1999, Takano et al., 2000, Wang et al., 2001, Genoler et al., 2004, Bensinger and Tontonoz, 2008, Szanto and Nagy, 2008). Forkhead box P3 is the transcription factor responsible for suppressive functions of Tregs. These cells are responsible for the generation and maintenance of maternal tolerance to fetal antigens. A decrease in Foxp3 expression could be due to loss of Tregs, or loss of their suppressive functions and conversion into effector T cells in response to exposure to PICs. Changes in quantity or function of Tregs leads to embryonic loss (Rowe et al., 2012b). Mannose receptor c type 1 is a receptor on МФs that is a marker for alternatively activated M2 MФs that produce Th2 cytokines compared to classically activated, pro-inflammatory M1 MФs that produce PICs (Gordon and Martinez, 2010). A loss in MRC1 expression suggests conversion of M2 to M1 MФs and increased production of PICs. When in animal has an infection, the goal is to clear the pathogen as soon as possible, this process is initiated with a strong inflammatory response. A decrease in these genes, which allows for an inflammatory response, therefore, is not unexpected. However, the inflammatory products produced and phenotype switching of immune cells from tolerogenic to immunogenic will put the embryo at risk.

With regard to Foxp3, there was a breed effect with Suffolk ewes having less expression, which fits with their enhanced inflammatory response. Fold change in gene expression on the experimental day was in reference to expression on the day of CIDR withdrawal ( 7 to 8 days prior) in the fall experiments. All of the Dorset ewes had an increase in Foxp3 between the two days (hour 0), but this increase did not 
occur in Suffolk ewes. Regulatory T cells increase in early pregnancy and accumulate in the uterus to establish maternal tolerance. This also results in increased Tregs in circulation. A lower fold change in Foxp3 expression in the Suffolk ewes at hour 0 may suggest differences in the generation of Tregs in early pregnancy in this breed, which is likely to be associated with increased rates of embryonic loss.

No effects of LPS on gene expression of IL-6, COX2, IFN $\gamma$, or IL-10 were detected. This does not mean a change in plasma protein levels of these mediators did not occur, however. While a change in gene expression of TNF $\alpha$ was not detected, protein levels of TNF $\alpha$ have been shown to increase after LPS treatment in early pregnant ewes (Graham, 2014). In addition, gene expression of NF- $\kappa$ B was not affected by LPS, but this transcription factor is downstream of TLR4, which was upregulated, and it turns on transcription of CXCL8, IL-6, IL-1 $\beta$, and TNF $\alpha$. Possibly, changes in gene transcription of NF-кB were

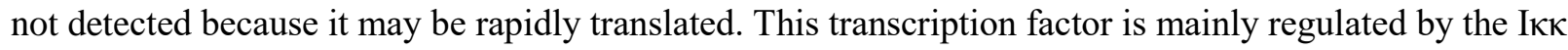
protein complex that holds it in the cytoplasm until activation of signal transduction pathways cause

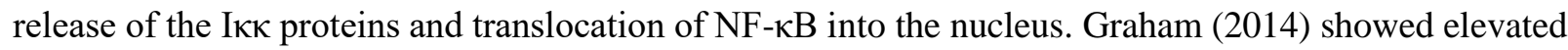
TNF $\alpha$ protein in response to LPS in ewes; current studies showed upregulation of CXCL8 expression; IL$1 \beta$ and IL-6 induce the fever response through activation of $\mathrm{PGE}_{2}$ in the brain, as well as activation of the HPA axis; and cortisol and IL-6 activate production of APPs, of which, Hp was increased in the current studies in response to LPS. Therefore, LPS likely increased production of TNF $\alpha$, IL-1 $\beta$, and IL-6. Additional studies should focus on measuring protein levels of these to get a clearer picture of the immune response to LPS in sheep. Measurement of TNF $\alpha$ and IL-1 $\beta$ protein expression using ELISA kits was unsuccessful. Future attempts should use radioimmunoassays instead.

Lipopolysaccharide always resulted in activation of the HPA axis and production of cortisol. Suffolk ewes had an enhanced production of cortisol in response to LPS, as did the flaxseed fed ewes in trial 2. Glucocorticoids have pro- and anti-inflammatory effects; they activate APP production, cause behavioral changes, and modulate cytokine production. Effects of LPS on TNF $\alpha$ and IL-6 gene expression were not detected, but protein levels of these cytokines are likely to have been increased as they are upstream of cortisol, the temperature response, and the sickness symptoms. Glucocorticoids can inhibit PIC production, but, associations between elevated cortisol levels and PIC concentrations were not determined. The consistent increase in Hp by LPS treatment suggests that cortisol was acting in a proinflammatory manner, however. The increase in Hp concentrations in the LPS treated ewes agrees with studies reporting that cows with uterine disease after parturition have increased production of $\mathrm{Hp}$ (Sheldon et al., 2001). Suffolk ewes had higher concentrations of the APP. This could be due to higher baseline levels, which could be beneficial in controlling bacterial growth, or Suffolk ewes may normally be in a more pro-inflammatory state compared to Dorset ewes. 
The complement system has a critical role in initiating inflammatory responses and activation of complement is known to result in pregnancy failure (Molina, 2005; Xu et al., 2000, Mellor et al., 2001, Mao et al., 2003; Caucheteux et al., 2003). LPS in the current studies increased expression of the complement molecule, C3, showing that complement is involved in mediating inflammation in this LPS model, and bacterial induced embryonic loss likely involves complement activation. LPS treated Suffolk ewes had an enhanced increase in C3 expression, which could lead to a greater inflammatory response in this breed. Indeed, even though Suffolk ewes had a lower expression of TLR4, they had a greater increase in temperature, "sickness symptoms," cortisol and Hp concentrations, and change in IL-6 and IFN $\gamma$ gene expression in response to LPS. This could be explained by increased complement activation and PIC production downstream of complement activation. Suffolk ewes also had a reduced change in PPAR $\gamma$ and Foxp3 expression, two transcription factors regulating anti-inflammatory functions, and the antiinflammatory cytokine IL-10. All of this information shows a more pro-inflammatory response in Suffolk ewes.

Embryotrophic factors produced by the oviduct and endometrium also are often immune modulators, including pro-inflammatory factors. Changes in the amount or temporal expression of these factors, such as during an infection, can affect embryonic development. The enhanced inflammatory response in Suffolk ewes will make them more susceptible to embryonic loss. Therapies that dampen the inflammatory response, therefore, will be more cost effective in Suffolk ewes that have higher rates of embryonic loss (Dixon et al., 2014). In addition, sheep producers may be interested in crossing the two breeds to try to get animals with a reduced inflammatory response compared to the Suffolk breed. In addition, studies investigating possible therapies for mastitis and reducing embryonic loss in dairy cows should be conducted using Suffolk ewes that have an exaggerated inflammatory response to LPS.

A possible reason for the breed differences may be modifications in the LPS-TLR4 interaction due to single nucleotide polymorphisms (SNPs) in TLR4. Susceptibility to bacterial infections in cattle has been associated with SNPs in TLR2, which led to changes in the immune response to pathogens (Koets et al., 2010). SNPs in TLR 2, 4, 6, and 9 have also been found in cattle but only a small effect was found on susceptibility to uterine infection (Pinedo et al., 2013). In order to investigate this possibility, TLR4 would need to be sequenced in the two breeds of sheep and compared

Prostaglandins are produced by COX enzymes, and LPS is known to increase production of $\mathrm{PGE}_{2}$ (Herath et al., 2009; Bromfield et al., 2015). Although no change in gene expression of COX2 in response to LPS was detected, PGs are mainly produced in tissues and not by circulating WBCs. Again, while it is likely that PGs were produced, measuring levels would confirm this and allow for the effects of the flaxseed supplement and breed to be determined. Suffolk ewes did have higher expression of COX2 compared to Dorset ewes, but whether they have increased $\mathrm{PGE}_{2}$ levels should be confirmed with 
measurement of protein. PICs induce $\mathrm{PGE}_{2}$ production by brain endothelial cells. The PG then acts on the hypothalamus to cause behavioral changes (Elenkov and Chrousos, 1999). In all 3 studies, LPS caused noticeable discomfort in ewes, further evidence of PIC production and COX pathway activation.

"Sickness behavior" was most pronounced in the Suffolk breed, also suggestive of increased PG production in these ewes.

Lipopolysaccharide consistently caused fever, which also is induced by $\mathrm{PGE}_{2}$ action on the hypothalamus (Netea et al., 2000; Evans et al., 2015). The enhanced temperature increase in the Suffolk ewes in response to LPS is likely to be a result of increased $\mathrm{PGE}_{2}$, as this breed had higher expression of COX2. Studies investigating heat stress have shown that elevated body temperature negatively impacts early embryonic development (Putney et al., 1988; Ealy et al., 1993), and the fever response to LPS likely has negative effects on embryo function. In trial 1 of the supplement study, feeding flaxseed dampened the fever response, but this was not the case in trial 2. A differential effect of flaxseed between the trials also occurred with gene expression of IL-6, IL-8, and TLR4 where in trial 1, a flaxseed x LPS interaction occurred with the increase in expression of the pro-inflammatory factors being dampened compared to control fed ewes. These effects were not seen in trial 2. This could be due to a lower fat content in the flaxseed used in trial 2, thus lower omega 3 PUFA intake. Another possiblility for the differential effects is grass content of $\omega-3$ PUFAs. In both the spring and fall trials, the ewes were on pasture and grass in the spring has a higher $\omega-3$ PUFA content than grass in the fall. Therefore, the ewes in trial 1 (spring)

probably ingested an increased amount of $\omega-3$ PUFAs. In order to have the same anti-inflammatory effects in trial 2 (fall) we probably needed to increase the amount of flaxseed being fed. Antiinflammatory effects of flaxseed should be investigated further by feeding different amounts of flaxseed, for various amounts of time prior to LPS treatment, to examine if there is a threshold intake level needed for beneficial effects on the inflammatory response. Flaxseed could reduce fever through altering the products of the COX pathway towards more pro-resolving mediators and less inflammatory PGs (Jones et al., 2013a, Jones et al., 2013b; Serhan et al., 2014). This could be especially important in the Suffolk ewes because they have higher COX2 expression and, therefore, may have increased PG production compared to Dorset ewes, therefore, possible anti-inflammatory effects of flaxseed should also be investigated in Suffolk ewes.

In conclusion, information gained from these studies suggest gram negative bacterial infections are likely to cause embryonic loss through the involvement of ROS, complement activation, heat damage to the embryo, production of PGs, production of PICs, conversion of Tregs to effector T cells, conversion of M2 to M1 MФs, and loss of maternal tolerance to the fetus. Differences in gene expression indicate Suffolk ewes are set up for a more rapid, intense, and prolonged inflammatory response compared to Dorset ewes and this enhanced response was observed when LPS was administered. The information 
gained with this research can be benefical to sheep producers deciding what breeds of sheep to raise and when making decisions about cross-breeding. In addition, knowledge of the immune response to LPS in sheep can be used to develop therapies for use by the dairy industry to combat economic losses associated with bacterial infections. 
Table 11: Summary of p-values across the three studies for main effects and interactions. The Benjamini Hochberg procedure was used to control for the false discovery rate (FDR=0.10)

\begin{tabular}{|c|c|c|c|c|c|c|c|c|}
\hline Variable & Experiment & LPS & $\begin{array}{l}\text { Feed or } \\
\text { Breed }\end{array}$ & Hour & $\begin{array}{l}\text { Feed or } \\
\text { Breed X } \\
\text { LPS }\end{array}$ & $\begin{array}{l}\text { Feed or } \\
\text { Breed } x \\
\text { Hour }\end{array}$ & $\begin{array}{l}\text { LPS } x \\
\text { Hour }\end{array}$ & $\begin{array}{l}\text { Feed or } \\
\text { Breed } \\
\text { X Hour } \\
\text { X LPS }\end{array}$ \\
\hline \multirow[t]{3}{*}{ Temperature } & $\begin{array}{l}\text { Supplement } \\
\text { Trial } 1\end{array}$ & $<0.001$ & $<0.001$ & $<0.001$ & 0.0064 & 0.6540 & $<0.001$ & 0.2683 \\
\hline & $\begin{array}{l}\text { Supplement } \\
\text { Trial } 2\end{array}$ & $<0.0001$ & 0.9942 & $<0.0001$ & 0.0164 & 0.1445 & $<0.0001$ & 0.3016 \\
\hline & Breed & 0.0001 & 0.0284 & $<0.0001$ & 0.0879 & 0.0330 & $<0.0001$ & 0.0862 \\
\hline \multirow[t]{3}{*}{ Symptoms } & $\begin{array}{l}\text { Supplement } \\
\text { Trial } 1\end{array}$ & $<0.0001$ & 0.0945 & & 0.0588 & & & \\
\hline & $\begin{array}{l}\text { Supplement } \\
\text { Trial } 2\end{array}$ & $<0.0001$ & 0.0498 & & 0.0498 & & & \\
\hline & Breed & $<0.0001$ & 0.3183 & & 0.0029 & & & \\
\hline \multirow[t]{3}{*}{ WBCs } & $\begin{array}{l}\text { Supplement } \\
\text { Trial } 1\end{array}$ & $<0.001$ & 0.0157 & 0.0039 & 0.8608 & 0.4827 & 0.0054 & 0.0056 \\
\hline & $\begin{array}{l}\text { Supplement } \\
\text { Trial } 2\end{array}$ & $<0.0001$ & 0.2284 & 0.0004 & 0.4034 & 0.5096 & $<0.0001$ & 0.9275 \\
\hline & Breed & $<0.0001$ & 0.3240 & $<0.0001$ & 0.3045 & 0.4592 & $<0.0001$ & 0.6694 \\
\hline \multirow[t]{3}{*}{ Haptoglobin } & $\begin{array}{l}\text { Supplement } \\
\text { Trial } 1\end{array}$ & $<0.0001$ & $<0.0001$ & & $<0.0001$ & & & \\
\hline & $\begin{array}{l}\text { Supplement } \\
\text { Trial } 2\end{array}$ & 0.0327 & 0.0982 & 0.0078 & 0.9681 & 0.2903 & 0.0165 & 0.0802 \\
\hline & Breed & 0.6804 & $<0.0001$ & 0.0405 & 0.0357 & 0.2366 & 0.2712 & 0.4907 \\
\hline \multirow[t]{3}{*}{$\begin{array}{l}\text { Serum } \\
\text { Amyloid A }\end{array}$} & $\begin{array}{l}\text { Supplement } \\
\text { Trial } 1\end{array}$ & $<0.0001$ & 0.0216 & & $<0.001$ & & & \\
\hline & $\begin{array}{l}\text { Supplement } \\
\text { Trial } 2\end{array}$ & 0.4771 & 0.4496 & 0.0023 & 0.0552 & 0.1324 & 0.5375 & 0.0844 \\
\hline & Breed & 0.0848 & 0.3228 & 0.3431 & 0.2221 & $<0.0001$ & 0.6979 & 0.0330 \\
\hline \multirow[t]{3}{*}{ Cortisol } & $\begin{array}{l}\text { Supplement } \\
\text { Trial } 1\end{array}$ & $<0.001$ & 0.0468 & & 0.8277 & & & \\
\hline & $\begin{array}{l}\text { Supplement } \\
\text { Trial } 2\end{array}$ & $<0.0001$ & 0.0235 & $<0.0001$ & 0.0007 & 0.1750 & $<0.0001$ & 0.0105 \\
\hline & Breed & 0.0029 & 0.0284 & $<0.0001$ & 0.0257 & 0.1262 & $<0.0001$ & 0.0085 \\
\hline \multicolumn{9}{|c|}{ Gene expression of pro-inflammatory factors } \\
\hline \multirow[t]{4}{*}{$\mathrm{C} 3$} & $\begin{array}{l}\text { Supplement } \\
\text { Trial } 1\end{array}$ & 0.0065 & 0.2651 & & 0.1020 & & & \\
\hline & $\begin{array}{l}\text { Supplement } \\
\text { Trial } 2\end{array}$ & 0.0107 & 0.0041 & $<0.0001$ & 0.2770 & 0.8603 & $<0.0001$ & 0.7626 \\
\hline & Breed & 0.0028 & 0.0077 & $<0.0001$ & 0.0004 & 0.3877 & $<0.0001$ & 0.5603 \\
\hline & & & & & & & & \\
\hline
\end{tabular}




\begin{tabular}{|c|c|c|c|c|c|c|c|c|}
\hline Variable & Experiment & LPS & $\begin{array}{l}\text { Feed or } \\
\text { Breed }\end{array}$ & Hour & $\begin{array}{l}\text { Feed or } \\
\text { Breed } x \\
\text { LPS }\end{array}$ & $\begin{array}{l}\text { Feed or } \\
\text { Breed } x \\
\text { Hour }\end{array}$ & $\begin{array}{l}\text { LPS x } \\
\text { Hour }\end{array}$ & $\begin{array}{l}\text { Feed or } \\
\text { Breed } x \\
\text { Hour } x \\
\text { LPS } \\
\end{array}$ \\
\hline \multirow[t]{3}{*}{ COX2 } & $\begin{array}{l}\text { Supplement } \\
\text { Trial } 1\end{array}$ & 0.1469 & 0.2671 & & 0.1748 & & & \\
\hline & $\begin{array}{l}\text { Supplement } \\
\text { Trial } 2\end{array}$ & 0.1497 & 0.3335 & 0.0119 & 0.7254 & 0.6789 & 0.1702 & 0.0262 \\
\hline & Breed & 0.2874 & 0.0002 & 0.7742 & 0.1441 & 0.8189 & 0.9809 & 0.5316 \\
\hline \multirow[t]{3}{*}{ IFNy } & $\begin{array}{l}\text { Supplement } \\
\text { Trial } 1\end{array}$ & 0.0024 & 0.3768 & & 0.8616 & & & \\
\hline & $\begin{array}{l}\text { Supplement } \\
\text { Trial } 2\end{array}$ & 0.2852 & 0.7478 & 0.1325 & 0.5615 & 0.9550 & 0.5091 & 0.5203 \\
\hline & Breed & 0.9118 & $\begin{array}{l}0.0153 \\
\end{array}$ & 0.4470 & 0.6391 & 0.5154 & 0.1554 & 0.1996 \\
\hline \multirow[t]{3}{*}{ IL-6 } & $\begin{array}{l}\text { Supplement } \\
\text { Trial } 1\end{array}$ & 0.3356 & 0.3186 & & 0.0296 & & & \\
\hline & $\begin{array}{l}\text { Supplement } \\
\text { Trial } 2\end{array}$ & 0.2174 & 0.2738 & 0.6169 & 0.9012 & 0.7886 & 0.4452 & 0.8527 \\
\hline & Breed & 0.5316 & 0.0007 & 0.7256 & 0.0024 & 0.9561 & 0.7193 & 0.8528 \\
\hline \multirow[t]{3}{*}{ IL-8 } & $\begin{array}{l}\text { Supplement } \\
\text { Trial } 1\end{array}$ & $<0.0001$ & 0.0486 & & 0.027 & & & \\
\hline & $\begin{array}{l}\text { Supplement } \\
\text { Trial } 2\end{array}$ & 0.0160 & 0.3185 & 0.1215 & 0.3238 & 0.3742 & 0.0990 & 0.2952 \\
\hline & Breed & 0.0003 & 0.5996 & 0.0005 & 0.4210 & 0.8304 & 0.0142 & 0.6080 \\
\hline \multirow[t]{3}{*}{ NF-kB } & $\begin{array}{l}\text { Supplement } \\
\text { Trial } 1\end{array}$ & 0.4731 & 0.90 & & 0.1283 & & & \\
\hline & $\begin{array}{l}\text { Supplement } \\
\text { Trial } 2\end{array}$ & 0.6009 & 0.2375 & 0.5398 & 0.9022 & 0.5339 & 0.4623 & 0.0563 \\
\hline & Breed & 0.9684 & 0.0518 & 0.4320 & 0.0443 & 0.6027 & 0.1815 & 0.1836 \\
\hline \multirow[t]{3}{*}{ TLR4 } & $\begin{array}{l}\text { Supplement } \\
\text { Trial } 1\end{array}$ & $<0.0001$ & 0.0484 & & 0.0326 & & & \\
\hline & $\begin{array}{l}\text { Supplement } \\
\text { Trial } 2\end{array}$ & 0.0002 & 0.5209 & 0.2734 & 0.0496 & 0.2734 & $<0.0001$ & 0.1865 \\
\hline & Breed & 0.0036 & $\begin{array}{l}0.0030 \\
\end{array}$ & 0.0121 & 0.0608 & 0.0572 & 0.0105 & 0.9920 \\
\hline \multirow[t]{3}{*}{ TNF $\alpha$} & $\begin{array}{l}\text { Supplement } \\
\text { Trial } 1\end{array}$ & 0.3482 & 0.5703 & & 0.3482 & & & \\
\hline & $\begin{array}{l}\text { Supplement } \\
\text { Trial } 2\end{array}$ & 0.6070 & 0.1603 & 0.2229 & 0.7149 & 0.7932 & 0.2600 & 0.2130 \\
\hline & Breed & 0.0608 & 0.4093 & 0.2390 & 0.6700 & 0.6566 & 0.2085 & 0.1443 \\
\hline \multicolumn{9}{|c|}{ Gene expression of anti-inflammatory factors } \\
\hline \multirow[t]{3}{*}{ Foxp3 } & $\begin{array}{l}\text { Supplement } \\
\text { Trial } 1\end{array}$ & 0.0259 & 0.8934 & & 0.2878 & & & \\
\hline & $\begin{array}{l}\text { Supplement } \\
\text { Trial } 2\end{array}$ & 0.1249 & 0.4214 & 0.2116 & 0.0914 & 0.0956 & 0.0242 & 0.4022 \\
\hline & Breed & 0.2861 & 0.0001 & 0.2819 & 0.5833 & 0.4560 & $<0.0001$ & 0.9459 \\
\hline
\end{tabular}




\begin{tabular}{|c|c|c|c|c|c|c|c|c|}
\hline Variable & Experiment & LPS & $\begin{array}{l}\text { Feed or } \\
\text { Breed }\end{array}$ & Hour & $\begin{array}{l}\text { Feed or } \\
\text { Breed } x \\
\text { LPS }\end{array}$ & $\begin{array}{l}\text { Feed or } \\
\text { Breed } x \\
\text { Hour }\end{array}$ & $\begin{array}{l}\text { LPS x } \\
\text { Hour }\end{array}$ & $\begin{array}{l}\text { Feed or } \\
\text { Breed } x \\
\text { LPS } x \\
\text { Hour }\end{array}$ \\
\hline \multirow[t]{3}{*}{ IL-10 } & $\begin{array}{l}\text { Supplement } \\
\text { Trial } 1\end{array}$ & 0.4274 & 0.0813 & & 0.8777 & & & \\
\hline & $\begin{array}{l}\text { Supplement } \\
\text { Trial } 2\end{array}$ & 0.2788 & 0.9603 & 0.0646 & 0.8639 & 0.9854 & 0.3456 & 0.3585 \\
\hline & Breed & 0.1459 & 0.0448 & 0.3033 & 0.7898 & 0.2340 & 0.8370 & 0.6515 \\
\hline \multirow[t]{3}{*}{ MRC1 } & $\begin{array}{l}\text { Supplement } \\
\text { Trial } 1\end{array}$ & $<0.0001$ & 0.8170 & & 0.6190 & & & \\
\hline & $\begin{array}{l}\text { Supplement } \\
\text { Trial } 2\end{array}$ & 0.0118 & 0.3273 & 0.0004 & 0.9326 & 0.9950 & 0.0091 & 0.9807 \\
\hline & Breed & 0.0028 & 0.5054 & 0.0003 & 0.5817 & 0.3018 & 0.1294 & 0.9159 \\
\hline \multirow[t]{3}{*}{ PPARY } & $\begin{array}{l}\text { Supplement } \\
\text { Trial } 1\end{array}$ & 0.1696 & 0.4523 & & 0.0702 & & & \\
\hline & $\begin{array}{l}\text { Supplement } \\
\text { Trial } 2\end{array}$ & 0.0014 & 0.2355 & 0.0436 & 0.9671 & 0.7764 & 0.6366 & 0.6932 \\
\hline & Breed & 0.0056 & 0.0016 & 0.0059 & 0.1483 & 0.9956 & 0.0054 & 0.5925 \\
\hline \multirow[t]{3}{*}{ SOD2 } & $\begin{array}{l}\text { Supplement } \\
\text { Trial } 1\end{array}$ & $<0.0001$ & 0.8022 & & 0.7071 & & & \\
\hline & $\begin{array}{l}\text { Supplement } \\
\text { Trial } 2\end{array}$ & $<0.0001$ & 0.0779 & $<0.0001$ & 0.0226 & 0.2068 & $<0.0001$ & 0.1794 \\
\hline & Breed & $<0.0001$ & 0.0002 & 0.0001 & 0.3674 & 0.4452 & $<0.0001$ & 0.5132 \\
\hline \multirow[t]{3}{*}{ TGF $\beta$} & $\begin{array}{l}\text { Supplement } \\
\text { Trial } 1\end{array}$ & & & & & & & \\
\hline & $\begin{array}{l}\text { Supplement } \\
\text { Trial } 2\end{array}$ & 0.1503 & 0.1003 & 0.0117 & 0.2196 & 0.8771 & 0.0691 & 0.2573 \\
\hline & Breed & 0.4916 & 0.0808 & 0.0161 & 0.5908 & 0.0144 & 0.1860 & 0.5186 \\
\hline \multirow[t]{3}{*}{$\mathrm{P}_{4} 25 / 26 \mathrm{dpc}$} & $\begin{array}{l}\text { Supplement } \\
\text { Trial } 1\end{array}$ & 0.6854 & 0.0752 & & 0.2026 & & & \\
\hline & $\begin{array}{l}\text { Supplement } \\
\text { Trial } 2\end{array}$ & 0.8523 & 0.4303 & & 0.1766 & & & \\
\hline & Breed & 0.2739 & 0.2137 & & 0.3254 & & & \\
\hline
\end{tabular}




\section{Chapter 6: References}

Abayasekara DRE. And Wathes DC. (1999) Effects of Altering Dietary Fatty Acid Composition on Prostaglandin Synthesis and Fertility. Prostaglandins, Leukotrienes, and Essential Fatty Acids. 61(5):275-287

Abrahams VM. (2008) Pattern Recognition at the Maternal-Fetal Interface. Immunological Investigations 37:427447

Abrahams VM., Kim YM., Straszewski SL., Romero R., Mor G. (2004) Macrophages and Apoptotic Cell Clearance during Pregnancy. American Journal of Reproductive Immunology 51:275-282

Abrahams VM., Visintin I., Aldo PB., Guller S., Romero R., Mor G. (2005) A Role for TLRs in the Regulation of Immune Cell Migration by First Trimester Trophoblast Cells. Journal of Immunology 175(12):8096-8104

Abumaree MH., Chamley LW., Badri M., El-Muzaini MF. (2012) Trophoblast Debris Modulates the Expression of Immune Proteins in Macrophages: A Key to Maternal Tolerance of the Fetal Allograft? Journal of_Reproductive immunology 94:131-141

Afonso PV., Janka-Junttila M., Lee YJ., McCann CP., Oliver CM., Aamer KA., Losert W., Cicerone MT., Parent CA. (2012) LTB $_{4}$ is a Signal-Relay Molecule during Neutrophil Chemotaxis. Developmental Cell 22(5):1079-1091

Agelaki S., Tsatsanis C., Gravanis A., Margioris AN. (2002) Corticotropin-Releasing Hormone Augments Proinflammatory Cytokine Production from Macrophages in Vitro and in Lipopolysaccharide-Induced Endotoxin Shock in Mice. Infection and Immunity 70(11):6068-6074

Agrawal V., Jaiswal MK., Jaiswal YK. (2011) Lipopolysaccharide Induces Alterations in Ovaries and Serum Level of Progesterone and 17 $\beta$ Estradiol in the Mouse. Fertility \& Sterility 95(4):1471-1474

Agrawal V., Jaiswal MK., Jaiswal YK. (2013a) LPS-Induced Implantation Failure: One of the Causes of Female Infertility. Medical Journal of Obstetrics and Gynecology 1(3):1014

Agrawal V., Jaiswal MK., Jaiswal YK. (2013b) Lipopolysaccharide-Induced Modulation in the Expression of Progesterone Receptor and Estradiol Receptor Leads to Early Pregnancy Loss in Mouse. Zygote 21(4):337-344

Akdis M., Burgler S., Crameri R., Eiwegger T., Fujita H., Gomez E., Klunker S., Meyer N., O’Mahony L., Palomares O., Rhyner C., Quaked N., Schaffartzik A., Van De Veen W., Zeller S., Zimmermann M., Akdis CA. (2011) Interleukins, From 1 to 37, and Interferon- $\gamma$ : Receptors, Functions, and Roles in Diseases. Journal of allergy and Clinical Immunology 127:701-721

Akira S., Hirano T., Taga T., Kishimoto T. (1990) Biology of Multifunctional Cytokines: IL 6 and Related Molecules (IL 1 and TNF). FASEB Journal 4(11):2860-2867

Akira S., Hoshino K. (2003) Myeloid Differentiation Factor 88-Dependent and -Independent Pathways in Toll-Like Receptor Signaling. Journal of Infectious Disease 187 (suppl 2): S356-S363

Akira S., Uematsu S., Takeuchi O. (2006) Pattern Recognition and Innate Immunity. Cell 124:783-801

Aliberti J., Hieny S., Reis e Sousa C., Serhan CN., Sher A. (2002) Lipoxin-Mediated Inhibition of IL-12 Production by DCs: A Mechanisms for Regulation of Microbial Immunity. Nature Immunology 3:76-82

Allen MS. (2000) Effects of Diet on Short-Term Regulation of Feed Intake by Lactating Dairy Cattle. Journal of Dairy Science 83(7):1598-1624 
Allison AC. (1958) The genetical and clinical significance of the haptoglobins. Proceedings of the Royal Soceity of Medicine 51(8):641-645

Alt C., Laschinger M., Engelhardt B. (2002) Functional Expression of the Lymphoid Chemokines CCL19 (ELC) and CCL21 (SLC) at the Blood-Brain Barrier Suggests their Involvement in G-Protein-Dependent Lymphocyte Recruitment into the Central Nervous System during Experimental Autoimmune Encephalomyelitis. European Journal of Immunology 32(8):2133-2144

Aluvihare VR., Killikourdis M., Betz AG. (2004) Regulatory T Cells Mediate Maternal Tolerance to the Fetus. Nature Immunology 5(3):266-271

Amano M., Galvan M., Baum LG. (2003) The ST6Gal I Sialyltransferase Selectively Modifies N-glycans on CD45 to Negatively Regulate Galectin-1-Induced CD45 Clustering, Phosphatase Modulation, and T Cell Death. Journal of Biological Chemistry 278(9):7469-7475

Ambrose DJ., Kastelic JP., Corbett R., Pitney PA., Petit HV., Small JA., Zalkovic P. (2006) Lower Pregnancy Losses in lactating Dairy Cows Fed a Diet Enriched in $\alpha$-Linolenic Acid. Journal of Dairy Science 89:3066-3074

Andrei C., Margiocco P., Poggi A., Lotti LV., Torrisi MR., Rubartelli A. (2004) Phospholipases C and A2 Control Lysosome-Mediated IL-1 $\beta$ Secretion: Implications for Inflammatory Processes. Proceedings of the National Academy of Sciences of The United States of America 101(26):9745-9750

Angioni S., Petraglia F., Gallinelli A., Cossarizza A., Franceschi C., Muscettola M., Genazzani AD., Surico N., Genazzani AR. (1993) Corticotropin-Releasing Hormone Modulates Cytokine Release in Cultured Human Peripheral Blood Mononuclear Cells. Life Sciences 53(23):1735-1742

Apetoh L., Quintana FJ., Pot C., Joller N., Xiao S., Kumar D., Burns EJ., Sherr DH., Weiner HL., Kuchroo VK. (2010) The Aryl Hydrocarbon Receptor Interacts with c-Maf to Promote the Differentiation of Type 1 Regulatory T Cells Induced by IL-27. Nature Immunology 11(9):854-861

Aplin JD., Meseguer M., Simon C., Ortíz E., Croxatto H., Jones CJP. (2001) MUC1, Glycans and the Cell-Surface Barrier to Embryo Implantation. Biochemical Society Transactions 29(Pt. 2):153-156

Appel S., Mirakaj V., Bringmann A., Weck MM., Grünebach F., Brossart P. (2005) PPAR-gamma Agonists Inhibit Toll-Like Receptor-Mediated Activation of Dendritic Cells via the MAP kinase and NF-kappa B Pathways. Blood 106(12):3888-3894

Arancibia SA., Beltrán CJ., Aguirre IM., silva P., Peralta AL., Malinarich F., Hermoso MA. (2007) Toll-Like Receptors are key Participants in Innate Immune Responses. Biological Research 40(2):97-112

Arck PC., Marali FS., Stanisz AM., Stead RH., Chaouat G., Manuel J., Clark DA. (1995) Stress-Induced Murine Abortion Associated with Substance P-Dependent Alteration in Cytokines in Maternal Uterine Decidua. Biology of Reproduction 53(4):814-819

Arck P., Hansen PJ., Jericevic BM., Piccinni M-P., Szekeres-Bartho J. (2007) Progesterone during Pregnancy: Endocrine-Immune Cross Talk in Mammalian Species and the Role of Stress. American Journal of Reproductive Physiology 58(3):268-279

Ariel A., Li PL., Wang W., Tang WX., Fredman G., Hong S., Gotlinger KH., Serhan CN. (2005) The Docosatriene Protectin D1 is produced by TH2 Skewing and Promotes Human T Cell Apoptosis via Lipid Raft Clustering. The Journal of Biological Chemistry 280(52):43079-43086

Ariel A., Fredman G., Sun YP., Kantarci A., Van Dyke TE., Luster AD., Serhan CN. (2006) Apoptotic Neutrophils and T Cells Sequester Chemokines during Immune Response Resolution through Modulation of CCR5 Expression. Nature Immunology 7(11):1209-1216 
Arita M., Bianchini F., Aliberti J., Sher A., Chiang N., Hong S., Yang R., Petasis NA., Serhan CN. (2005) Stereochemical Assignment, Antiinflammatory Properties, and Receptor for the Omega-3 Lipid Mediator Resolvin E1. The Journal of Experimental Medicine 201(5):713-722

Arita M., Ohira T., Sun Y-P., Elangovan S., Chiang N., Serhan CN. (2007) Resolvin E1 Selectively Interacts with Leukotriene $\mathrm{B}_{4}$ Receptor BLT1 and ChemR23 to Regulate Inflammation. Journal of Immunology 178(6):3912-3917

Armstrong, J.D., Goodall, E.A., Gordon, F.J., Rice, D.A., McCaughey, W.J. (1990) The Effects of Levels of Concentrate and Inclusion of Maize Gluten or Fish Meal in the Concentrate on Reproductive Performance and Blood Parameters of Dairy Cows. Animal Production 50:1-10

Arosh JA., Banu SK., Kimmins S., Chapdelaine LA., Maclaren LA., Fortier MA. (2004) Effect of Interferon-Tau on Prostaglandin Biosynthesis, Transport, and Signaling at the Time of Maternal Recognition of Pregnancy in Cattle: Evidence of Polycrine Actions of Prostaglandin E2. Endocrinology 145:5280-5293

Arredouani MS., Kasran A., Vanoirbeek JA., Berger FG., Baumann H., Ceuppens JL. (2005) Haptoglobin dampens Endotoxin-Induced Inflammatory effects both in vitro and in vivo. Immunology 114(2):263-271

Ashkar AA., Di Santo JP., Croy BA. (2000) Interferon gamma Contributes to Initiation of Uterine Vascular Modification, Decidual Integrity, and Uterine Natural Killer Cell Maturation during Normal Murine Pregnancy. Journal of Experimental Medicine 192(2):259-270

Asseman C., Mauze S., Leach MW., Coffman RL., Powrie F. (1999) An Essential Role for Interleukin 10 in the Function of Regulatory T Cells that Inhibit Intestinal Inflammation. Journal of Experimental Medicine 190(7):9951004

Athanassakis I., Aifantis I., Baritkis S., Farmakiotis V., Koumantakis E., Vassiliadis S. (2000) Nitric oxide Production by Pre-Implantation Embryos in Response to Embryotoxic Factors. Cellular Physiology and Biochemistry 10:169-176

Awasthi A., Carrier Y., Peron JPS., Bettelli E., Kamanaka M., Flavell RA., Kuchroo VK., Oukka M., Weiner HL. (2007) A Dominant Function for Interleukin 27 in Generating Interleukin 10-Producing Anti-Inflammatory T Cells. Nature Immunology 8:1380-1389

Baban B., Chandler PR., Sharma MD., Pihkala J., Koni PA., Munn DH., Mellor A. (2009) IDO Activates Regulatory T Cells and Blocks their Conversion into Th-17 like T Cells. Journal of Immunology 183(40:2475-2483

Badinga L., Gülay MS., Ealy A. (2016) CLA and EPA Inhibit LPS-Induced Prostaglandin Release from Bovine Endometrial cells Through an NF-кB-Dependent Signaling Mechanism. Turkish Journal of Veterinary and Animal Sciences 40:382-388

Bagavandoss P., Wiggins RC., Kunkel SL., Remick DG., Keyes PL. (1990) Tumor Necrosis Factor Production and Accumulation of Inflammatory Cells in the Corpus Luteum of Pseudopregnancy and Pregnancy in Rabbits. Biology of Reproduction 42(2):367-376

Banerjee DK., Dhodapkar MV., Matayeva E., Steinman RM., dhodapkar KM. (2006) Expansion of FOXP3 high Regulatory T cells by Human Dendritic cells (DCs) in vitro and after Injection of Cytokine-Matured DCs in Myeloma Patients. Blood 108(8):2655-2661

Bankhurst AD. (1982) The Modulation of Human Natural Killer Cell Activity by Prostaglandins. Journal of Clinical \& Laboratory Immunology 7(2):85-91

Bannenberg G. and Serhan CN. (2010) Specialized Pro-Resolving Lipid Mediatros in the Inflammatory Response: an Update. Biochimica et Biophysica acta 1801(12):1260-1273 
Bar D., Tauer LW., Bennett G., González RN., Hertl JA., Schukken YH., Schulte HF., Welcome FL., Gröhn YT. (2008) The Cost of Generic Clinical Mastitis in Dairy Cows as Estimated by Using Dynamic Programming. Journal of Dairy Science 91:2205-2214

Barak Y., Sadovsky Y., Shalom-Barak T. (2008) PPAR Signaling in Placental Development and Function. PPAR Research 142082

Baratelli F., Lin Y., Zhu L., Yang SC., Heuzé-Vourch N., Zeng G., Reckamp K., Dahadwala M., Sharma S., Dubinett SM. (2005) Prostaglandin E2 Induces FOXP3 Gene Expression and T Regulatory Cell Function in Human CD4+ T Cells. Journal of Immunology 175(3):1483-1490

Barker AR., Schrick FN., Lewis MJ., Dowlen HH., Oliver SP. (1998) Influence of Clinical Mastitis during Early Lactation on Reproductive Performance of Jersey Cows. Journal of Dairy Science 81(5):1285-1290

Bauernfeind FG., Horvath G., Stutz A., Alnemri ES., MacDonald K., Speert D., Fernandes-Alnemri T., Wu J., Monks BG., Fitzgerald KA., Hornung V., Latz E. (2009) Cutting Edge: NF- $\mathrm{B}$ Activating pattern Recognition and Cytokine Receptors License NLRP3 Inflammasome Activation by Regulating NLRP3 Expression. Journal of Immunology 183:787-791

Baum LG., Pang M., Perillo NL., Wu T., Delegeane A., Uittenbogaart CH., Fukuda M., Seilhamer JJ. (1995) Human Thymic Epithelial Cells Express an Endogenous Lectin, Galectin-1, which Binds to Core O-Glycans on Thymocytes and T Lymphoblastoid Cells. Journal of Experimental Medicine 18(3):877-887

Bauman DE., Currie WB. (1980) Partitioning of Nutrients during Pregnancy and Lactation: A Review of mechanisms Involving Homeostasis and Homeorhesis. Journal of Dairy Science 63:1514-1529

Baybutt HN. And Holsboer F. (1990) Inhibition of Macrophage Differentiation and Function by Cortisol. Endocrinology 127:476-480

Bazer FW (1992) Mediators of Maternal Recognition of Pregnancy in Mammals. Proceedings of the Society for Experimental Biology and Medicine 199(4):373-384

Bazer FW (2013) Pregnancy Recognition Signaling Mechanisms in Ruminants and Pigs Journal of Animal Science and Biotechnology 4:23

Bazer FW., Burghardt RC., Johnson GA., Spencer TE., Wu G. (2008) Interferons and Progesterone for Establishment and Maintenance of Pregnancy: Interactions among Novel Cell Signaling Pathways. Reproductive Biology 8(3):179-211

Bazer FW., Spencer TE., Johnson GA., Burghardt RC., WU G. (2009a) Comparative Aspects of Implantation. Reproduction 138:195-209

Bazer FW., Spencer TE., Johnson GA. (2009b) Interferons and Uterine Receptivity. Seminars in Reproductive Medicine 27:90-102

Bazer FW., Wu G., Spencer TE., Johnson GA., Burghardt RC., Bayless K. (2010) Novel Pathways for Implantation and Establishment and Maintenance of Pregnancy in Mammals. Molecular Human Reproduction 16(3):135-152

Bellingan GJ., Caldwell H., Howie SE., Dransfield I., Haslett C. (1996) In Vivo Fate of the Inflammatory Macrophage during the Resolution of Inflammation; Inflammatory Macrophages do not Die Locally, but Emigrate to the Draining Lymph Nodes. Journal of Immunology 157(6):2577-2585

Bensinger SJ. and Tontonoz P. (2008) Integration of Metabolism and Inflammation by Lipid-Activated Nuclear Receptors. Nature 454(7203):470-477

Berger J. and Moller DE. (2002) The Mechanisms of Action of PPARs. Annual Review of Medicine 53:409-435 
Bergsbaken T., Fink SL., Cookson BT. (2009) Pyroptosis: Host Cell Death and Inflammation. Nature Reviews. Microbiology 7(2):99-109

Berkenbosch F., van Oers J., del Ray A., Tilders F., Besedovsky H.(1987) Corticotropin-Releasing FactorProducing Neurons in the Rat Activated by Interleukin-1. Science 238(4826):524-526

Bernardini R., Kamilaris TC., Calogero AE., Johnson EO., Gomex MT., Gold PW., Chrousos GP. (1990) Interactions between Tumor Necrosis Factor- $\alpha$, Hypothalamic Corticotropin-Releasing Hormone, and Adrenocorticotropin Secretion in the Rat. Endocrinology 126(6):2876-2881

Bettelli E., Carrier Y., Gao W., Korn T., Strom TB., Oukka M., Weiner HL., Kuchroo VK. (2006) Reciprocal Developmental Pathways for the Generation of Pathogenic Effector $\mathrm{T}_{\mathrm{H}} 17$ and Regulatory T Cells. Nature 441(7090):235-238

Betz M. and Fox BS. (1991) Prostaglandin E2 Inhibits Production of Th1 Lymphokines but not of Th2 Lymphokines. Journal of Immunology 146:108-113

Bilby TR., Sozzi A., Lopez MM., Silvestre FT., Ealy AD., Staples CR., Thatcher WW. (2006a) Pregnancy, Bovine Somatotropin, and Dietary n-3 Fatty Acids in Lactating Dairy Cows: I. Ovarian, Conceptus, and Growth HormoneInsulin-Like Growth Factor System Responses. Journal of Dairy Science 89:3360-3374

Bilby TR., Jenkins T., Staples CR., Thatcher WW. (2006c) Pregnancy, Bovine Somatotropin, and Dietary n-3 Fatty Acids in Lactating Dairy Cows: III. Fatty Acid Distribution. Journal of Dairy Science 89:3386-3399

Biron CA., Nguyen KB., Pien GC., Cousens LP., Salazar-Mather TP. (1999) Natural Killer Cells in Antiviral Defense: Functions and Regulation by Innate Cytokines. Annual Review of Immunology 17:189-220

Bischof P., Meisser A., Campana A. (2000) Mechanisms of Endometrial Control of Trophoblast Invasion. Journal of Reproduction and Fertility. Supplement 55:65-71

Blaser C., Kaufmann M., Müller C., Zimmermann C., Wells V., Mallucci L., Pircher H. (1998) Beta-GalactosideBinding Protein Secreted by Activated T Cells Inhibits Antigen-Induced Proliferation of T Cells. European Journal of Immunology 28(8):2311-2319

Blidner AG. and Rabinovich GA. (2013) 'Sweetening' Pregnancy: Galectins at the Fetomaternal Interface. American Journal of Reproductive Immunology 69:369-382

Blois SM., Alba Soto CD., Tometten M., Klapp BF., Margni RA., Arck PC. (2004a) Lineage, Maturity, and Phenotype of Uterine Murine Dendritic Cells throughout Gestation Indicate a Protective Role in Maintaining Pregnancy. Biology of Reproduction 70(4):1018-1023

Blois SM., Joachim R., Kandil J., Margni R., Tometten M., Klapp BF., Arck PC. (2004b) Depletion of CD8+ Cells Abolishes the Pregnancy Protective Effect of Progesterone Substitution with Dydrogesterone in Mice by Altering the Th1/Th2 Cytokine Profile. Journal of Immunology 172(10):5893-5899

Blois S., Tometten M., Kandil J., Hagen E., Klapp BF., Margni RA., Arck PC. (2005) Intercellular Adhesion Molecule-1/LFA-1 Cross Talk is a Proximate Mediator Capable of Disrupting Immune Integration and Tolerance Mechanism at the Feto-Maternal Interface in Murine Pregnancies. Journal of Immunology 174(4):1820-1829

Blois SM., Kammerer U., Alba Soto C., Tometten MC., Shaikly V., Barrientos G., Jurd R., Rukavina D., Thomson AW., Klapp BF., Fernández N., Arck PC. (2007a) Dendritic Cells: Key to Fetal Tolerance? Biology of Reproduction 77(4):590-598

Blois S.M., Ilarregui J.M., Tometten M., Garcia M., Orsal A.S., Cordo-Russo R., Toscano M.A., Bianco G.A., Kobelt P., Handjiski B., Tirado I., Markert U.R., Klapp B.F., Poirier F., Szekeres-Bartho, J., Rabinovich G.A., Arck P.C. (2007b) A Pivotal Role for Galectin-1 in Fetomaternal Tolerance Nature Medicine 13(12):1450-1457 
Bogdan C. and Nathan C. (1993) Modulation of Macrophage Function by Transforming Growth Factor beta, Interleukin-4, and Interleukin-10. Annals of the New York Academy of Sciences 685:713-739

Bondurant RH. (1999) Inflammation in the Bovine Female Reproductive Tract. Journal of Animal Science 77(Suppl 2):101-110

Bopp T., Becker C., Klein M., Klein-Hessling S., Palmetshofer A., Serfling E., Heib V., Becker M., Kubach J., Schmitt S., Stoll S., Schild H., Staege MS., Stasson M., Jonuleit H., Schmitt E. (2007) Cyclic Adenosine Monophosphate is a Key Component of Regulatory T Cell Mediated Suppression. Journal of Experimental Medicine 204(6):1303-1310

Borgeat P. and Naccache PH. (1990) Biosynthesis and Biological Activity of Leukotriene B4. Clinical Biochemistry 23(5):459-468

Borregaard N. (2010) Neutrophils, from Marrow to Microbes. Immunity 33(5):657-670

Boshier DP. (1969) A Histological and Histochemical Examination of Implantation and Early Placentome Formation in Sheep. Journal of Reproduction and Fertility 19:51-91

Boshier DP., Fairclough RJ., Holloway H. (1987) Assessment of Sheep Blastocyst Effects on Neutral Lipids in the Uterine Caruncular Epithelium. Journal of Reproduction and Fertility 79:569-573

Boumpas DT., Chrousos GP., Wilder RL., Cupps TR., Balow JE. (1993) Glucocorticoid Therapy for ImmuneMediated Diseases: Basic and Clinical Correlates. Annals of Internal Medicine 119(12):1198-1208

Bousserouel S., Brouillet A., Bereziat G., Raymondjean M., Andreani M. (2003) Different Effects of n-6 and n-3 Polyunsaturated Fatty Acids on the Activation of Rat Smooth Muscle Cells by Interleukin-1 $\beta$. Journal of Lipid Research 44:216-221

Boussiotis VA., Chen ZM., Zeller JC., Murphy WJ., Berezovskaya A., Narula S., Roncarolo MG.,Blazar BR. (2001) Altered T-Cell Receptor + CD28-Mediated Signaling and Blocked Cell Cycle Progression in Interleukin 10 and Transforming Growth Factor-beta Treated Alloreactive T Cells that do Not Induce Graft-Versus-Host Disease. Blood 97(2):565-571

Bray MA., Cunningham FM., Ford-Hutchinson AW., Smith MJ. (1981) Leukotriene B4: a Mediator of Vascular Permeability. British Journal of Pharmacology 72(3):483-486

Brayman MJ., Julian J., Mulac-Jericevic B., Conneely OM., Edwards DO., Carson DD. (2006) Progesterone Receptor Isoforms A and B Differentially Regulate MUC1 Expression in Uterine Epithelial Cells. Molecular Endocrinology 20(10):2278-2291

Breder CD., Dinarello CA., Saper CB. (1988) Interleukin-1 Immunoreactive Innervation of the Human Hypothalamus. Science 240(4850):321-324

Bright JJ. and Sriram S. (1998) TGF beta Inhibits IL-12 Induced Activation of Jak-STAT Pathway in T Lymphocytes. Journal of Immunology 161(4):1772-1777

Brinsfield TH. Anf Hawk HW. (1973) Control by Progesterone of the Concentration of Lipid Droplets in Epithelial Cells of the Sheep Endometrium. Journal of Animal Science 36:919-922

Bromfield JJ., Santos JEP., Block J., Williams RS., Sheldon IM. (2015) Physiology and Endocrinology Symposium: Uterine Infection: Linking Infection and Innate Immunity with Infertility in the High-Producing Dairy Cow. Journal of Animal Science 93:2021-2033

Brooks KE., Burns GW., Spencer TE. (2015) Peroxisome Proliferator Activator Receptor Gamma (PPARG) Regulates Conceptus Elongation in Sheep. Biology of Reproduction 92(2):42,1-13 
Brown WJ., Chambers K., Doody A. (2003) Phospholipase A(2) (PLA(2)) Enzymes in Membrane Trafficking: Mediators of membrane Shape and Function. Traffic 4(4):214-221

Buford WI., Ashmad N., Schrick FN., Butcher RL., Lewis PE., Inskeep EK. (1996) Embryotoxicity of a Regressing Corpus Luteum in Beef Cows Supplemented with Progestogen. Biology of Reproduction 54:531-537

Burke, J.M., Staples, C.R., Risco, C.A., De La Sota, R.L., Thatcher, W.W. (1997) Effect of Ruminant Grade Menhaden Fish Meal on Reproductive and Productive Performance of Lactating Dairy Cows. Journal of Dairy Science 80:3386-3398

Burns PD., Engle TE., Harris MA., Enns RM., Whittier JC. (2003) Effect of Fish Meal Supplementation on Plasma and Endometrial Fatty Acid Composition in Nonlactating Beef Cows. Journal of American Science 81:2840-2846

Butts CL., Shukair SA., Duncan KM., Bowers E., Horn C., Belyavskaya E., Tonelli L., Sternbery EM. (2007) Progesterone Inhibits Mature Rat Dendritic Cells in a Receptor-Mediated Fashion. International Immunology 19(3);287-296

Byrne A. and Reen DJ. (2002) Lipopolysaccharide Induces Rapid Production of IL-10 by Monocytes in the Presence of Apoptotic Neutrophils. Journal of Immunology 168(4):1967-1977

Caldari-Torres C., Rodriquez-Sallaberry C., Greene ES., badinga L. (2006) Differential Effects of n-3 and n-6 Fatty Acids on Prostaglandin $F_{2 \alpha}$ Production by Bovine Endometrial Cells. Journal of Dairy Science 89:971-977

Calder PC. (1996) Immunomodulatory and Anti-Inflammatory effects on n-3 Polyunsaturated Fatty Acids. Proceedings of the Nutrition Society 55:737-774

Calder PC. (1997) n-3 Polyunsaturated Fatty Acids and Cytokine Production in Health and Disease. Annals of Nutrition and Metabolism 41(4):203-234

Calder PC. (1998) Fat Chance of Immunomodulation. Immunology Today 19(6):244-247

Calder PC. (2001) Polyunsaturated Fatty Acids, Inflammation, and Immunity. Lipids 36(9):1007-1024

Calder PC. (2002) Dietary Modification of Inflammation with Lipids. Proceedings of the Nutrition Society 61:345358

Calder PC (2003) N-3 Polyunsaturated Fatty Acids and inflammation: From Molecular Biology to the Clinic. Lipids 38(4):343-352

Calder PC. (2006a) Polyunsaturated Fatty Acids and Inflammation. Prostaglandins, Leukotrienes, and Essential Fatty Acids 75(3):197-202

Calder PC. (2006b) n-3 Polyunsaturated Fatty Acids, Inflammation, and Inflammatory Diseases. American Journal of Clinical Nutrition 83: 1505S-1519S

Calder PC. (2011) Fatty Acids and Inflammation: The Cutting Edge between Food and Pharma. European Journal of Pharmacology 668:S50-S58

Calder PC (2012) Long-Chain Fatty Acids and Inflammation. Proceedings of the Nutrition Society 71:284-289

Calder PC. (2013a) Long Chain Fatty Acids and Gene Expression in Inflammation and Immunity. Current Opinion in Clinical Nutrition and Metabolic Care 16(4):425-433

Calder PC. (2013b) n-3 Fatty Acids, Inflammation and Immunity: New Mechanisms to Explain Old Actions. The Proceedings of Nutrition Society 72(3):326-336

Calder PC. (2013c) Omega-3 Polyunsaturated Fatty Acids and Inflammatory Processes: Nutrition or Pharmacology? British Journal of Clinical Pharmacology 75:645-662 
Cammas L., Reinaud P., Borda N., Dubois O., Germain G., Charpigny G. (2006) Developmental Regulation of Prostacyclin Synthase and Prostacylin Receptors in the Ovine Uterus and Conceptus during the Peri-Implantation Period. Reproduction 75:289-296

Canny G., Levy O., Furuta GT., Narravula-Alipati S., Sisson RB., Serhan CN., Colgan SP. (2002) Lipid MediatorInduced Expression of Bactericidal/Permeability-Increasing Protein (BPI) in Human Mucosal Epithelia. Proceedings of the National Academy of Sciences of the United States of America 99(6):39002-3907

Care AS., Diener KR., Jasper MJ., Brown HM., Ingman WV., Robertson SA. (2013) Macrophages Regulate Corpus Luteum Development during Embryo Implantation in Mice. Journal of Clinical Investigation 123(8):3472-3487

Carlino C., Stabile H., Morrone S., Bulla R., Soriani A., Agostinis C., Bossi F., Mocci C., Sarazani F., Tedesco F., Santoni A., Gismondi A. (2008) Recruitment of Circulating NK Cells through Decidual Tissues: a Possible mechanism Controlling NK cell Accumulation in the Uterus during Early Pregnancy. Immunobiology 111:31083115

Carneiro LC., Cronin JG., Sheldon IM. (2016) Mechanisms Linking Bacterial Infections of the Bovine Endometrium to Disease and Fertility. Reproductive Biology 16:1-7

Caroprese M., Ciliberti MG., Annicchiarico G., Albenzio M., Muscio A., Sevi A. (2014) Hypothalamic-PituitaryAdrenal Axis Activation and Immune Regulation in Heat-Stressed Sheep after Supplementation with Polyunsaturated Fatty Acids. Journal of Dairy Science 97:4247-4258

Caroprese M., Ciliberti MG., Albenzio M., Annicchiarico G., Sevi A. (2015) Dietary Polyunsaturated Fatty Acids from Flaxseed affect Immune Responses of Dairy Sheep around Parturition. Veterinary Immunology and Immunopathology 168:56-60

Carter AM. (2012) Evolution of Placental Function in Mammals: the Molecular Basis of Gas and Nutrients Transfer, Hormone Secretion, and Immune Responses. Physiological Reviews 92:1543-1576

Cassatella MA., Gasperini S., Bovolenta C., Calzetti F., Vollebregt M., Scapini P., Marchi M., Suzuki R., Suzuki A., Yoshimura A. (1999) Interleukin-1o (IL-10) Selectively Enhances CIS3/SOCS3 mRNA Expression in Human Neutrophils: Evidence for an IL-10-Induced Pathway that is Independent of STAT Protein Activation. Blood 94(8):2880-2889

Castrillo A. and Tontonoz P. (2004) Nuclear Receptors in Macrophage Biology: At the Crossroads of Lipid Metabolism and Inflammation. Annual Review of Cell and Developmental Biology 20:455-480

Caucheteux SM., Kanellopoulos-Langevin C., Ojcius DM. (2003) At the Innate Frontiers between Mother and Fetus: Linking Abortion with Complement Activation. Immunity 18(2):169-172

Caughey, G.E., Mantzioris, E., Gibson, R.A., Cleland, L.G., James, M.J. (1996) The Effect on Human Tumor Necrosis Factor $\alpha$ and Interleukin $1 \beta$ Production of Diets Enriched in n-3 Fatty Acids from Vegetable Oil or Fish Oil. American Journal of Clinical Nutrition 63:116-122

Caux C., Massacrier C., Vanbervliet B., Barthelemy C., Liu YJ., Banchereau J. (1994) Interleukin 10 Inhibits T Cell Alloreaction Induced by Human Dendritic Cells. International Immunology 6(8):1177-1185

Ceciliani F., Giordano A., Spagnolo V. (2002) The Systemic Reaction During Inflammation: The Acute-Phase Proteins. Protein and Peptide Letters 9(3):211-223

Cedeno-Laurent F., Opperman M., Barthel SR., Kuchroo VK., Dimitroff CJ. (2012) Galectiin-1 Triggers an Immunoregulatory Signature in Th Cells Functionally Defined by Il-10 Expression. Journal of Immunology 188:3127-3137 
Cerdeira AS., Rajakumar A., Royle CM., Lo L., Husain Z., Thadhani RI., Sukhatme VP., Karumanchi SA., Kopcow HD. (2013) Conversion of Peripheral Blood NK Cells to a Decidual NK-like Phenotype by a Cocktail of Defined Factors. The Journal of Immunology 190: 3939-3948

Chamley LW., Chen Q., Ding J., Stone PR., Abumaree M. (2011) Trophoblast Deportation: Just a Waste Disposal System or Antigen Sharing? Journal of Reproductive Immunology 88:99-105

Chang HY., Lee H-N., Kim W., Surh Y-J. (2015) Docosahexaenoic Acid Induces M2 Macrophage Polarization through Peroxisome Proliferator-Activated Receptor $\gamma$ Activation. Life Sciences 120:39-47

Chaouat G., Menu E., Clark DA., Dy M., Minkowski M., Wegmann TG. (1990) Control of Fetal Survival in CBAxDBA/2 Mice by Lymphokine Therapy. Journal of Reproduction and Fertility 89(2):447-458

Chaouat G., Assal Meliani A., Martal J., Raghupathy R., Elliot J., Mosmann T., Wegmann TG. (1995) IL-10 Prevents Naturally Occurring Fetal Loss in the CBAxDBA/2 Mating Combination, and Local Defect in IL-10 Production in the Abortion-Prone Combination is Corrected by in vivo Injection of IFN-tau. Journal of Immunology 154(9):4261-4268

Chaouat G., Zourbas S., Ostojic S., Lappree-Delage G., Dubanchet S., Ledee N., Martal J. (2002) A Brief Review of Recent Data on Some Cytokine Expressions at the Materno-Foetal Interface which Might Challenge the Classical TH1/TH2 Dichotomy. Journal of Reproductive Immunology 53(1-2):241-256

Chapwanya A., Meade KG., Doherty ML., Callanan JJ., O’Farrelly C. (2013) Endometrial Epithelial Cells are Potent Producers of Tracheal Antimicrobial Peptide and Serum Amyloid A3 Gene Expression in Response to E. Coli Stimulation. Veterinary Immunology and Immunopathology 151(1-2):157-162

Charpigny G., Reinaud P., Tamby JP., Créminon C., Martal J., Maclouf J., Guillomot M. (1997a) Expression of Cyclooxygenase-1 and -2 in Ovine Endometrium During the Estrous Cycle and Early Pregnancy. Endocrinology 138(5):2163-2171

Charpigny G., Reinaud P., Tamby JP., Créminon C., Guillomot M (1997b) Cyclooxygenase-2 unlike Cyclooxygenase-1 is highly expressed in Ovine Embryos during the Implantation Period. Biology of Reproduction 57:1032-1040

Chauhan SS., Celi P., Fahri FT., Leury BJ., Dunshea FR. (2014) Dietary Antioxidants at Supranutritional Doses Modulate Skeletal Muscle Heat Shock Protein and Inflammatory Gene Expression in Sheep Exposed to heat Stress. Journal of Animal Science 92(11):4897-4908

Chebel RC., Santos JEP., Reynolds JP., Cerri RLA., Juchem SO., Overton M. (2004) Factors Affecting Conception Rate after Artificial Insemination and Pregnacy Loss in Lactating Dairy Cows. Animal Reproduction Science $84: 239-255$

Chen W. and Wahl SM. (2002) TGF-beta: Receptors, Signaling Pathways and Autoimmunity. Current Directions in Autoimmunity 5:62-91

Chen GY. and Nuñez G. (2010) Sterile Inflammation: Sensing and Reacting to Damage. Nature Reviews: Immunology 10(12):826-837

Chen W., Wenwen J., Wahl SM. (1998) Engagement of Cytotoxic T Lymphocyte-Associated Antigen 4 (CTLA-4) Induces Transforming Growth factor beta (TGF-beta) Production by Murine CD4(+) T Cells. Journal of Experimental Medicine 188(10):1849-1857

Chen F., Castranova V., Shi X., Demers LM. (1999) New Insights into the Role of Nuclear Factor-Kappa B, a Ubiquitous Transcription Factor in the Initiation of Diseases. Clinical Chemistry 45:7-17 
Chen CH., Sequin-Devaux C., Burke NA., Oriss TB., Watkins SC., Clipstone N., Ray A. (2003) Transforming Growth Factor beta Blocks Tec Kinase Phosphorylation, Ca2+ Influx, and NFATc Translocation Causing Inhibition of T Cell Differentiation. Journal of Experimental Medicine 197(12):1689-1699

Chen W., Jin W., Hardegen N., Lei KJ., Li L., Marinos N., McGrady G., Wahl SM. (2003) Conversion of Peripheral CD4+CD25- Naïve T Cells to CD4+CD25+ Regulatory T Cells by TGF-beta Induction of Transcription Factor Foxp3. Journal of Experimental Medicine 198(12):1875-1886

Cheville N. (1988) Pathogenesis of Acute Inflammation. Pages 309-330 in Introduction to Veterinary Pathology. Iowa State University. Press, Ames, IA

Chiang N,. Fredman G., Bäckhed F., Oh SF., Vickery T., Schmidt BA., Serhan CN. (2012) Infection Regulates ProResolving Mediators that Lower Antibiotic Requirements. Nature 484(7395):524-528

Chiang N., Dalli J., Colas RA., Serhan CN. (2015) Identification of Resolvin D2 Receptor Mediating Resolution of Infections and Organ Protection. Journal of Experimental Medicine 212(8):1203-1217

Chin PY., Macpherson AM., Thompson JG., Lane M., Robertson SA. (2009) Stress Response Genes are suppressed in Mouse Preimplantation Embryos by granulocyte-Macrophage Colony-Stimulating Factor (GM-CSF). Human Reproduction 24(12):2997-3009

Chin PY., Thompson JG., Robertson SA. (2011) Maternal Interleukin-10 Deficiency Increases Sensitivity to Adverse Programming Effects of a Low Dose LPS Insult in the Pre-Implantation Period. Biology of Reproduction 85(Suppl1): 183

Ching S., Zhang H., Belevych N., He L., Lai W., Pu XA., Jaeger LB., Chen Q., Quan N. (2007) Endothelial-Specific Knockdown of Interleukin-1 (IL-1) type 1 Receptor differentially Alters CNS Responses to IL-1 depending on its Route of Administration. Journal of Neuroscience 27(39)"10476-10486

Chiu CY., Gomolka B., Dierkes C., Huang NR., Schroeder M., Purschke M., Manstein D., Dangi B., Weylandt KH. (2012) Omega-6 Docosapentaenoic Acid-Derived Resolvins and 17-Hydroxydocosahexaenoic Acid Modulate Macrophage Function and Alleviate Experimental Colitis. Inflammation Research 61(9):967-976

Choe YS., Shim C., Choi D., Lee CS., Lee K-K., Kim K. (1997) Expression of Galectin-1 mRNA in the Mouse Uterus is Under the Control of Ovarian Steroids during Blastocyst Implantation. Molecular Reproduction and Reproduction 48(2):261-266

Chou RC., Kim ND., Sadik CD., Seung E., Lan Y., Byrne MH., Haribabu B., Iwakura Y., Luster AD. (2010) LipidCytokine-Chemokine Cascade Drives Neutrophils Recruitment in a Murine Model of Inflammatory Arthritis. Immunity 33(2):266-278

Chow JC., Young DW., Golenbock DT., Christ WJ., Gusovsky F. (1999) Toll-Like Receptor-4 Mediates Lipopolysaccharide-Induced Signal Transduction. Journal of Biological Chemistry 274(16):10689-10692

Christman JW., Lancaster LH., Blackwell TS. (1998) Nuclear Factor Kappa B: A Pivotal Role in the Systemic Inflammatory Response Syndrome and New Target for Therapy. Intensive Care Medicine 24(11):1131-1138

Chrousos GP. (1995) The Hypothalamic-Pituitary-Adrenal Axis and Immune-Mediated Inflammation. New England Journal of Medicine 332(20):1351-1362

Chung CD., Patel VP., Moran M., Lewis LA., Miceli MC. (2000) Galectiin-1 Induces Partial TCR $\zeta$-Chain Phosphorylation and Antagonizes Processive TCR Signal Transduction. Journal of Immunology 165:3722-3729

Cipolletta D., Feuerer M., Li A., Kamei N., Lee J., Shoelson SE., Banoist C., Mathis D. (2012) PPAR- $\gamma$ is a Major Driver of the Accumulation and Phenotype of Adipose Tissue Treg Cells. Nature 486(7404):549-553 
Clark DA., Merali FS., Hoskin DW., Steel-Norwood D., Arck PC., Croitoru K., Murgita RA., Hirte H. (1997) Decidua-Associated Suppressor Cells in Abortion-Prone DBA/2 Mated CBA/J Mice that Release Bioactive Transforming Growth Factor $\beta 2$ Related Immunosuppressive Molecule Express a Bone-Marrow-Derived Natural Suppressor Cell Marker and $\gamma \delta$ T Cell Receptor. Biology of Reproduction 56:1351-1360

Clark RB., Bishop-Bailey D., Estrada-Hernandez T., Hla T., Puddington L., Padula SJ. (2000) The Nuclear Receptor PPAR Receptor Gamma and Immunoregulation: PPAR Gamma Mediates Inhibition of Helper T Cell Responses.

Clark DA., Fernandez J., Banwatt D., (2008) Prevention of Spontaneous Abortion in the CBAxDBA/2 Mouse Model by Intravaginal TGF-beta and Local Recruitment of CD4+CD8+ FOXP3+ cells. American Journal of Reproductive Immunology 59(6):525-534

Clark DA. and Chaouat G. (2012) Regulatory T Cells and Reproduction: How do they do it? Journal of Reproductive Immunology 96:1-7

Clifton VL., Owens PC., Robinson PJ., Smith R. (1995) Identification and Characterization of a CorticotrophinReleasing Hormone Receptor in Human Placenta. European Journal of Endocrinology 133(5):591-597

Cobb SP. and Watson ED. (1995) Immunohistochemical Study of Immune Cells in the Bovine Endometrium at Different Stages of the Oestrous Cycle. Research in Veterinary Science 59(3):238-241

Colas RA., Shinohara M., Dalli J., Chiang N., Serhan CN. (2014) Identification and Signature Profiles for ProResolving and Inflammatory Lipid Mediators in Human Tissue. American Journal of Physiology. Cell Physiology 307:C39-54

Committee on Bovine Reproductive Nomenclature (1972) Recommendations for Standardizing Bovine Reproductive Terms. Cornell Veterinarian 216-237

Contini P., Ghio M., Poggi A., Filaci G., Indiveri F., Ferrone S., Puppo F. (2003) Soluble HLA-A,-B,-C and -G Molecules Induce Apoptosis in T and NK CD8 Cells and Inhibit Cytotoxic T cell Activity through CD8 Ligation. European Journal of Immunology 33:125-134

Cooper KE., Cranston WI., Snell ES. (1964) Temperature Regulation during Fever in Man. Clinical Science 27:345356

Corey EJ., Shih C., Cashman JR. (1983) Docosahexaenoic Acid is a Strong Inhibitor of Prostaglandin but not Leukotriene Biosynthesis. Proceedings of the National Academy of Sciences of the United States of America. 80(12):3581-3584

Côrtes C., de Silva-Kazama DC., Kazama R., Gagnon N., Benchaar C., Santos GTD., Zeoula LM., Petit HV. (2010) Milk Composition, Milk Fatty Acid Profile, Digestion, and Ruminal Fermentation in Dairy Cows Fed Whole Flaxseed and Calcium Salts of Flaxseed Oil. Journal of Dairy Science 93(7):3146-3157

Corthay A. (2009) How do Regulatory T Cells Work? Scandinavian Journal of Immunology 70(4):326-336

Costello MJ., Joyce SK., Abrahams VM. (2007) NOD Protein Expression and Function in First Trimester Trophoblast Cells. American Journal of Reproductive Immunology 57:67-80

Cray C., Zaias J., Altman NH. (2009) Acute Phase Response in Animals: A Review. Comparative Medicine 59(6):517-526

Crncic TB., Laskarin G., Frankovic KJ., Tokmadzic VS., Strbo N., Bedenicki L., Le Bouteiller P., Tabiasco J., Rukavina D. (2007) Early Pregnancy Decidual Lymphocytes beside Perforin use Fas ligand (FasL) Mediated Cytotoxicity. Journal of Reproductive Immunology 73(2):108-117

Cronstein BN., Kimmel SC., Levin RI., Martiniuk F., Weissmann G. (1992) A Mechanism for the Antiinflammatory Effects of Corticosteroids: the Glucocorticoid Receptor Regulates Leukocyte Adhesion to Endothelial Cells and 
Expression of Endothelial-Leukocyte Adhesion Molecule 1 and Intracellular Adhesion Molecule 1. Proceedings of the National Academy of Science of the United States of America 89(21):9991-9995

Cross JC. (2005) How to Make a Placenta: Mechanisms of Trophoblast Cell Differentiation in Mice-A Review. Placenta 26(Suppl A):S3-S9

Croy BA., van den Heuvel MJ., Borzychowsi AM., Tayade C. (2006) Uterine Natural Killer Cell: A Specialized Differentiation Regulated by Ovarian Hormones. Immunology Reviews 214:161-185

Curti A., Trabanelli S., Salvestrini V., Baccarani M., Lemoli RM., (2009) The Role of Indoleamine 2,3-Dioxygenase in the Induction of Immune Tolerance: Focus on Hematology. Blood 113:2394-2401

Curtis CL., Hughes CE., Flannery CR., Little CB., Harwood JL., Caterson B. (2000) n-3 Fatty Acids Specifically Modulate Catabolic Factors Involved in Articular Cartilage Degradation. Journal of Biological Chemistry 275(2):721-724

Curtis CL., Rees SG., Little CB., Flannery CR., Hughes CE., Wilson C., Dent CM., Otterness IG., Harwood JL., Caterson B. (2002) Pathologic Indicators of Degradation and Inflammation in Human Osteoarthritic Cartilage are Aborgated by Exposure to n-3 Fatty Acids. Arthritis and Rheumatism 46(6):1544-1553

Dalli J., Cloas RA., Serhan CN. (2013) Novel n-3 Immunoresolvents: Structures and Actions. Scientific Reports $3: 1940$

Dalli J., Chiang N., Serhan CN. (2015) Elucidation of Novel 13-Series Resolvins that Increase with Atorvastatin and Clear Infections. Nature Medicine 21(9):1071-1075

Darasse-Jéze G., Klatzmann D., Charlotte F., Salomon BL., Cohen JL. (2006) CD4+CD25+ Regulatory/Suppressor T Cells Prevent Allogeneic Fetus Rejection in Mice. Immunology Letters 102:106-109

Dasu MR., Park S., Devaraj S., Jialal I. (2009) Pioglitazone Inhibits Toll-Like Receptor Expression and Activity in Human Monocytes and db/db Mice. Endocrinology 150:3457-3464

Davidson JA., Tiemann U., Betts JG., Hansen PJ. (1995) DNA Synthesis and Prostaglandin Secretion by Bovine Endometrial Cells as Regulated by Interleukin-1. Reproduction, Fertility, and Development 7(5):1037-1043

Davies CJ., Hill JR., Edwards JL., Schrick FN., Fisher PJ., Eldridge JA., Schlafer DH. (2004) Major Histocompatibility Antigen Expression on the Bovine Placenta: its Relationship to Abnormal Pregnancies and Retained Placenta. Animal Reproductive Science 82-83:267-280

Davies CJ., Eldridge JA., Fisher PJ., Schlafer DH. (2006) Evidence for Expression of both Classical and Nonclassical Major Histocompatibility Complex Class I genes in Bovine Trophoblast Cells. American Journal of Reproductive Immunology 55(3):188-200

Davies D., Meade KG., Herath S., Eckersall PD., Gonzalez D., White JO., Conlan RS., O'Farrelly C. Sheldon IM. (2008) Toll-Like Receptor and Antimicrobial Peptide Expression in the Bovine Endometrium. Reproductive Biology and Endocrinology 6:53

Daynes RA. and Jones DC. (2002) Emerging Roles of PPARs in Inflammation and Immunity. Nature Reviews Immunology 2(10):748-759

Deaglio S., Dwyer KM., Gao W., Friedman D., Usheva A., Erat A., Chen JF., Enjyoji K., Linden J., Oukka M., Kuchroo VK., Strom TB., Robson SC. (2007) Adenosine Generation Catalyzed by CD39 and CD73 Expressed on Regulatory T Cells Mediates Immune Suppression. Journal of Experimental Medicine 204(6):1257-1265

Deb K., Chaturvedi MM., Jaiswal YK. (2004) Comprehending the Role of LPS in Gram-Negative Bacterial Vaginosis: Ogling into the Causes of Unfulfilled Child-Wish. Archives of Gynecology and Obstetrics 270(3):133146 
de Caterina R., Cybulsky MI., Clinton SK., Gimbrone MA., Libby P. (1994) The Omega-3 Fatty Acid

Docsahexaenoate Reduces Cytokine-Induced Expression of Proatherogenic and Proinflammatory Proteins in Human Endothelial Cells. Arteriosclerosis and Thrombosis: A Journal of Vascular Biology 14(11):1829-1836

de Caterina R. and Libby P. (1996) Control of Endothelial Leukocyte Adhesion Molecules by Fatty Acids. Lipids Suppl:S57-63

de Waal Malefyt R., Haanen J., Spits H., Roncarolo MG., te Velde A., Figdor C., Johnson K., Kastelein R., Yssel H., de Vries JE. (1991) IL-10 and Viral IL-10 Strongly Reduce Antigen-Specific Human T Cell Proliferation by Diminishing the Antigen-Presenting Capacity of Monocytes via Downregulation of Class II MHC Expression. Journal of Experimental Medicine 174(4):915-924

Degrelle SA,. Campion E., Cabau C., Piumi F., Reinaud P., Richard C., Renard J-P., Hue I. (2005) Molecular Evidence for a Critical Period in Mural Trophoblast Development in Bovine Blastocyst. Developmental Biology 288:448-460

Denison FC., Grant VE., Calder AA., Kelly RW. (1991) Seminal Plasma Components Stimulate Interleukin-8 and Interleukin-10 Release. Molecular Human Reproduction 5(3):220-226

Dennis EA. And Norris PC. (2012) Omega-3 Fatty Acids Cause Dramatic Changes in TLR4 and Purinergic Eicosanoid Signaling. Proceedings of the National Academy of Sciences of the United States of America 109(22):8517-8522

Dennis EA. And Norris PC. (2015) Eicosanoid Storm in Infection and Inflammation. Nature Reviews. Immunology $15: 511-523$

Desai N., Scarrow M., Lawson J., Kinzer D., Goldfarb J. (1999) Evaluation of the Effect of Interleukin-6 and Human Extracellular Matrix on Embryonic Development. Human Reproduction 41(6):1588-1592

Dey SK. (2005) Fatty Link to Fertility. Nature 435:34-35

Dey SK., Lim H., Sanjoy K., Das JR., Paria BC., Daikoku T., Wang H. (2004) Molecular Cues to Implantation. Endocrine Reviews 25(3):341-373

D'Hauterive PS., Charlet-Renard C., Dubois M., Berndt S., Goffin F., Foidart JM., Geenen V. (2005) Human Endometrial Leukemia Inhibitory Factor and Interleukin-6: Control of Secretion by Transforming Growth Factor beta Related members. Neuroimmunomodulation 12(3):157-163

Dimitriadis E., White CA., Jones RL., Salamonsen LA. (2005) Cytokines, Chemokines, and Growth Factors in Endometrium Related to Implantation. Human Reproduction Update 11(6):613-630

Dinarello CA. (2007) Historical Review of Cytokines. European Journal of Immunology 37(Suppl 1) : S34-S45

Dinarello CA. (2009) Immunological and Inflammatory Functions of the Interleukin-1 Family. Annual Reviews of Immunology 27:519-550

Dinarello CA. (2010) Anti-Inflammatory Agents: Present and Future. Cell 140(6):935-950

Ding L., Linsley PS., Huang LY., Germain RN., Shevach EM. (1993) IL-10 Inhibits Macrophage Costimulatory Activity by Selectively Inhibiting the Up-Regulation of B7 Expression. Journal of Immunology 151(3):1224-1234

Diskin MG., Murphy JJ., Sreenan JM. (2006) Embryo Survival in Dairy Cows Managed Under Pastoral Conditions. Animal Reproduction Science 96:297-311

Diskin MG., Morris DG. (2008) Embryonic and Early Foetal losses in Cattle and Other Ruminants. Reproduction of Domestic Animals 43(suppl. 2):260-267 
Dorniak P., Bazer FW., Spencer TE. (2011) Prostaglandins Regulate Conceptus Elongation and Mediate Effects of Interferon Tau on the Ovine Uterine Endometrium. Biology of Reproduction 84:1119-1127

Dorniak P., Bazer FW., Wu G., Spencer TR. (2012a) Conceptus- Derived Prostaglandins Regulate Endometrial Function in Sheep. Biology of Reproduction 87(1):9:1-7

Dorniak P., Welsh TH., Bazer FW., Spencer TR. (2012b) Endometrial HSD11B1 and Cortisol Regeneration in the Ovine Uterus: Effects of Pregnancy, Interferon Tau, and Prostaglandins. Biology of Reproduction 86(4):124,1-10

Dorniak P., Bazer FW., Spencer TR.(2013a) Physiology and Endocrinology Symposium: Biological Role of Interferon Tau in Endometrium Function and Conceptus Elongation. Journal of Animal Science 91:1627-1638

Dorniak P., Welsh TH., Bazer FW., Spencer TR. (2013b) Cortisol and Interferon Tau Regulation of Endometrial Function and Conceptus Development in Female Sheep. Endocrinology 154:931-941

Dostál J., Oborná I., Talas M., Chrastinová L., Machovská K. (1996) Infectious Agents and Treatment of Infertility with IVF and ET. Ceska Gynekologie 61(3):144-147

Dow TL., Rogers-Nieman G., Holásková I., Elasser TH., Dailey DA. (2010) Tumor Necrosis Factor-alpha and Acute-Phase Proteins in Early Pregnant Ewes after Challenge with Peptidoglycan-Polysaccharide. Domestic Animal Endocrinology 39(2):147-154

Du MR., Guo PF., Piao HL., Wang SC., Sun C., Jin LP., Tao Y., Li Y., Zhang D., Zhu R., Fu Q., Li D. (2014) Embryonic Trophoblasts Induce Decidual Regulatory T Cell Differentiation and Maternal-Fetal Tolerance through Thymic Stromal Lymphopoietin Instructing Dendritic cells. The Journal of Immunology 192:1502-1511

Duffield JS., Ware CF., Ryffel B., Savill J. (2001) Suppression by Apoptotic cells Defines Tumor Necrosis FactorMediated Induction of Glomerular Mesangial Cell Apoptosis by Activated Macrophages. The American Journal of Pathology 195(4):1397-1404

Dumoulin JC., Menheere PP., Evers JL., Kleukers AP., Pieters MH., Bras M., Geraedts JP. (1991) The Efffects of Endotoxins on Gametes and Preimplantation Embryos Cultured in Vitro. Human Reproduction 6(5):730-734

Duriez M., Quilley H., Madec Y., El Costa H., Cannou C., Marlin R., de Truchis C., Rahmati M., Barré-Sinoussi F., Nugeyre M., Menu E. (2014) Human Decidual Macrophages and NK Cells Differentially Express Toll-Like

Receptors and Display Distinct Cytokine Profiles upon TLR Stimulation. Frontiers in Microbiology 5:316-329

Ealy AD., Drost M., Hansen PJ. (1993) Developmental Changes in Embryonic Resistance to Adverse Effects of Maternal heat Stress in Cows. Journal of Dairy Science 76:2899-2905

Eaton JW., Brandt P., Mahoney JR., Lee JT JR. (1982) Haptoglobin: a Natural Bacteriostat. Science 215(4533):691693

Eckersall PD. and Bell R. (2010) Acute Phase Proteins: Biomarkers of Infection and Inflammation in Veterinary Medicine. Veterinary Journal 185:23-27

Eggleston DL., Wilken C., Van Kirk EA., Slaughter RG., Ji TH., Murdoch WJ. (1990) Progesterone Induces Expression of Endometrial Messenger RNA Encoding for Cyclooxygenase (sheep). Prostaglandins 39:675-683

Elenkov IJ. And Chrousos GP. (1999) Stress Hormones, Th1/Th2 Patterns, Pro/Anti-Inflammatory Cytokines and Susceptibility to Disease. Trends in Endocrinology and Metabolism 10(9):359-368

Elenkov IJ., Papanicolaou DA., Wilder RL., Chrousos GP. (1996) Modulatory Effects of Glucocorticoids and Catecholamines on Human IL-12 and Interleukin-10 Production. Proceedings of the Association of American Physicians 108(5):374-381 
Elenkov IJ., Webster EL., Torpy DJ., Chrousos GP. (1999) Stress, Corticotropin-Releasing Hormone, glucocorticoids, and the Immune/Inflammatory Response: Acute and Chronic Effects. Annals of the New York Academy of Sciences 876:1-11

El-Kebir D., Gjorstrup P., Filep JG. (2012) Resolvin E1 Promotes Phagocytosis-Induced Neutrophil Apoptosis and Accelerates Resolution of Pulmonary Inflammation. Proceedings of the National Academy of Sciences of the United States of America. 109(37):14983-14988

El-Sayed A., Hoelker M., Rings F., Salilew D., Jennen D., Tholen E., Sirard M-A., Schellander K., Tesfaye D. (2006) Large-Scale Transcriptional Analysis of Bovine Embryo Biopsies in Relation to Pregnancy Success after Transfer to Recipients. Physiological Genomics 28:84-96

Elsasser T., Kahl S., MacLeod C., Nicholson B., Sartin JL., Li C. (2004) Mechanisms Underlying Growth Hormone Effects in Augmenting Nitric Oxide Production and Protein Tyrosine Nitration during Endotoxin Challenge.

Endocrinology 145(7):3413-3423

Endres A., Ghorbani R., Kelley VE., Georgilis K., Lonnemann G., Van Der Meer JMW., Cannon JG., Rogers TS., Klempner MS., Weber PC., Schaefer EJ., Wolff SM., Dinarello CA. (1989) The Effect of Dietary Supplementation with n-3 Polyunsaturated Fatty Acids on the Synthesis of Interleukin-1 and Tumor Necrosis Factor by Mononuclear Cells. The New England Journal of Medicine 320(5): 265-271

Engblom D., EK M., Saha S., Ericsson-Dahlstrand A., Jakobsson PJ., Blomqvist A. (2002) . Prostaglandins as Inflammatory Messengers Across the Blood-Brain Barrier. Journal of Molecular Medicine 80:5-15

Engelhardt B. (2006) Molecular Mechanisms Involved in T Cell Migration Across the Blood-Brain Barrier. Journal of Neural Transmission 113(4):477-485

Engering AJ., Cella M., Fluitsma D., Brockhaus M., Hoefsmit ECM., Lanzavecchia A., Pieters J. (1997) The Mannose Receptor Functions as a High Capacity and Broad Specificity Antigen Receptor in uman Dendritic Cells. European Journal of Immunology 27:2417-2425

Engström L., Ruud J., Eskilsson A., Larsson A., Mackerlova L., Kugelberg U., Qian H., Vasilache AM., Larsson P., Engblom D., Sigvardsson M., Jönsson JI., Blomqvist A. (2012) Lipopolysaccharide-Induced Fever Depends on Prostagladin E2 Production Specifically in Brain Endothelial Cells. Endocrinology 153(10):4849-4861

Eriksson M., Meadows SK., Wira CR., Sentman CL. (2004) Unique Phenotype of Human Uterine NK Cells and their Regulation by Endogenous TGF-beta. Journal of Leukocyte Biology 76(3):667-675

Eriksson M., Meadows SK., Wira CR., Sentman CL. (2006) Endogenous Transforming Growth Factor-beta Inhibits Toll-Like Receptor Mediated Activation of Human Uterine Natural Killer Cells. American Journal of Reproductive Immunology 56(5-6):321-328

Erlebacher A., Zhang D., Parlow AF., Glimcher LH. (2004) Ovarian Insufficiency and Early Pregnancy Loss Induced by Activation of the Innate Immune System. Journal of Clinical Investigation 114:39-48

Erlebacher A. (2013) Immunology of the Maternal-Fetal Interface. Annual Reviews of Immunology 31:387-411

Eskilsson A., Mirrasekhian E., Dufour S., Schwaninger M., Engblom D., Blomqvist A. (2014) Immune-Induced Fever is Mediated by IL-6 Receptors on Brain Endothelial Cells Coupled to STAT3-Dependent Induction of Brain Endothelial Prostaglandin Synthesis. Journal of Neuroscience 34(48):15957-15961

Espinoza J., Erez O., Romero R. (2006) Preconceptional Antibiotic Treatment to Prevent Preterm Birth in Women with a Previous Preterm Delivery. American Journal of Obstetrics and Gynecology 194(3):630-637

Evans AC. And Walsh SW. (2012) The Physiology of Multifactorial Problems Limiting the Establishment of Pregnancy in Dairy Cows. Reproduction, Fertility, and Development 24:233-237 
Evans SS., Repasky EA., Fisher DT. (2015) Fever and the Thermal Regulation of Immunity: The Immune System Feels the Heat. Nature Reviews Immunology 15:335-349

Ezashi T., Ealy AD., Ostrowski MC., Roberts RM. (1998) Control of Interferon- $\tau$ Gene Expression by Ets-2. Proceedings of the National Academy of Sciences of the United States of America 95(14):7882-7887

Fabian D., Juhás S., Il'ková G., Koppel J. (2007) Dose and Time Dependent Effects of TNFalpha and Actinomycin D on Cell Death Incidence and Embryo Growth in Mouse Blastocyst. Zygote 15(3):241-249

Fadok VA., Bratton DL., Konowal A., Freed PW., Westcott JY., Henson PM. (1998) Macrophages that have Ingested Apoptotic Cells in Vitro Inhibit Proinflammatory Cytokine Production through Autocrine/Paracrine Mechanisms Involving TGF-beta, PGE2, and PAF. Journal of Clinical Investigations 101(4):890-898

Fairchild DL.,Pate JL. (1991) Modulation of Bovine Luteal Cell Synthetic Capacity by Interferon-gamma. Biology of Reproduction 44(2):357-363

Fallarino F., Grohmann U., Vacca C., Bianchi R., Orabona C., Spreca A., Fioretti MC., Puccetti P. (2002) T Cell Apoptosis by Tryptophan Catabolism. Cell Death and Differentiation 9(10):1069-1077

Fallarino F., Groshmann U., Hwang KW., Orabona C., Vacca C., Bianchi R., Belladonna ML., Fioretti MC., Alegre M-L., Puccetti P. (2003) Modulation of Tryptophan Catabolism by Regulatory T Cells. Nature Immunology 4(12):1206-1212

Fang X., Wong S., Mitchell BF. (2000) Effects of LPS and IL-6 on Oxytocin Receptor in Non-Pregnant and Pregnant Rat Uterus. American Journal of Reproductive Immunology 44(2):65-72

Fantini MC., Becker C., Monteleone G., Pallone F., Galle PR., Neurath MF. (2004) Cutting Edge: TGF-beta Induces a Regulatory Phenotype in CD4+CD25- T Cells Through Foxp3 Induction and Down-Regulation of Smad7. Journal of Immunology 179(9):5149-5153

Farmer JL., Burghardt RC., Jousan FD., Hansen PJ., Bazer FW., Spencer TE. (2008) Galectin-15 (LGASL15) Functions in Trophectoderm Migration and Attachment. FASEB 22:548-560

Farran TB., Reinhardt CD., Blasi DA., Minton JE., Elsasser TH., Higgins JJ., Drouillard JS. (2008) Source of Dietary Lipid may modify the Immune Response in Stressed Feeder Cattle. Animal Science 86:1382-1394

Faveeuw C., Fougeray S., Angeli V., Fontaine J., Chinetti G., Gosset P., Delerive P., Maliszewski C., Capron M., Staels B., Moser M., Trottein F. (2000) Peroxisome Proliferator-Activated Receptor gamma Activators Inhibit Interleukin-12 Production in Murine Dendritic Cells. FEBS Letters 486(3):261-266

Feige JN., Gelman L., Michalik L., Desvergne B., Wahli W. (2006) From Molecular Action to Physiological Outputs: Peroxisome Proliferator-Activated Receptors are Nuclear Receptors at the Crossroads of Key cellular Functions. Progress in Lipid Research 45(2):120-159

Fernandis AZ. and Wenk MR. (2007) Membrane Lipids as Signaling Molecules. Current Opinion in Lipidology $18: 121-12$

Fenton MJ., Buras JA., Donnelly RP. (1992) IL-4 Reciprocally Regulates IL-1 and IL-1 Receptor Antagonist Expression in Human Monocytes. Journal of Immunology 149(4):1283-1288

Ferrara N., Gerber H-P., LeCouter J. (2003) The Biology of VEGF and its Receptors. Nature Medicine 9:669-676

Ferrari D., Chiozzi P., Falzoni S., Dal Susino M., Melchiorri L., Baricordi OR.,Virgilio FD. (1997) Extracellular ATP Triggers IL-1 beat Release by Activating the Purinergic P2z Receptor of Human Macrophages. Journal of Immunology 159(3):1451-1458

Ferrari D., Pizzirani C., Adinolfi E., Lemoli RM., Curti A., Idzko M., Panther E., Virgilio FD. (2006) The P2X 7 Receptor: A Key Player in IL-1 Processing and Release. Journal of Immunology 179(12):8569 
Fest S., Aldo PB., Abrahams VM., Visintin I., Alvero A., Chen R., Chavez SL., Romero R., Mor G. (2007) Trophoblast-Macrophage Interations: A Regulatory Network for the Protection of Pregnancy. American Journal of Reproductive Immunology 57:55-66

Fink SL. and Cookson BT. (2006) Caspase-1-Dependent Pore Formation during Pyroptosis Leads to Osmotic Lysis of Infected Host Macrophages. Cellular Microbiology 8(11):1812-1825

Fiorentino DF., Zlotnik A., Mosmann TR., Howard M., Moore KW., O’Garra A. (1991a) IL-10 Inhibits Cytokine Production by Activated Macrophages. Journal of Immunology 147(11):3815-3822

Fiorentino DF., Zlotnik A., Vieira P., Mosmann TR., Howard M., O'Garra A. (1991b) IL-10 Acts on the AntigenPresenting Cell to Inhibit Cytokine Production by Th1 Cells. Journal of Immunology 146(10):3444-3451

Foldi J., Kulcsar M., Pecsi A., Huyghe B., de Sa C., Lohuis JA., Cox P., Huszenicza G. (2006) Bacterial Complications of Postpartum Uterine Involution in Cattle. Animal Reproduction Science 96:265.281

Fontenot JD., Gavin MA., Rudensky AY. (2003) Foxp3 Programs the Development and Function of CD4+CD25+ Regulatory T Cells. Nature Immunology 4:330-336

Forman BM., Tontonoz P., Chen J., Brun RP., Spiegelman BM., Evans RM. (1995) Deoxy-delta 12,14prostaglandin $\mathrm{J} 2$ is a Ligand for the Adipocyte Determination factor PPAR gamma. Cell 83(5):803-812

Fourichon C., Seegers H., Malher X. (2000) Effects of Disease on Reproduction in the Dairy Cow. A MetaAnalysis. Theriogenology 53:1729-1759

Fournel S., Aguerre-Girr M., Huc X., Lenfant F., Alam A., Toubert A., Bensussan A., Le Bouteiller P. (2000) Cutting Edge: Soluble HLA-G1 Triggers CD95/CD95 Ligand Mediated Apoptosis in Activated CD8 Cells by Interacting with CD8. Journal of Immunology 164(12):6100-6104

Fournier T., Tsatsaris V., Handschuh K., Evain-Brion D. (2007) PPARs and the Placenta. Placenta 28:65-76

Fournier T., Guibourdenche J., Handschuh K., Tsatsaris V., Rauwel B., Davrinche C., Evain-Brion D. (2011) PPAR $\gamma$ and Human Trophoblast Differentiation. Journal of Reproductive Immunology 90:41-49

Francisco LM., Salinas VH., Brown KE., Vanquri VK., Freeman GJ., Kuchroo VK., Sharpe AH. (2009) PD-L1 Regulates the Development, Maintenance, and Function of Induced Regulatory T Cells. Journal of Experimental Medicine 206(13):3015-3029

Fredman G., Li Y., Dalli J., Chiang N., Serhan CN. (2012) Self-Limited versus Delayed Resolution of Acute Inflammation: Temporal Regulation of Pro-Resolving Mediatros and MicroRNA. Scientific Reports 2:269

Friebe A., Douglas AJ., Solano E., Blois SM., Hagen E., Klapp BF., Clark DA., Arck PC. (2011) Neutralization of LPS or Blockage of TLR4 Signaling Prevents Stress-Triggered Fetal Loss in Murine Pregnancy. Journal of Molecular Medicine 89:689-699

Frumento G., Rotondo R., Tonetti M., Damonte G., Benatti U., Ferrara GB. (2002) Tryptophan-Derived Catabolites are Responsible for Inhibition of $\mathrm{T}$ and Natural Killer Cell Proliferation Induced by Indoleamine 2,3-dioxygenase. Journal of Experimental Medicine 196(40:459-468

Fu B., Li X., Sun R., Tong X., Ling B., Tian Z., Wei H. (2013) Natural Killer Cells Promote Immune Tolerance by Regulating Inflammatory TH17 Cells at the Human Maternal-Fetal Interface. Proceedings of the National Academy of Sciences of the U.S.A. 110:E231-E240

Fullerton JN., O'Brien AJ., Gilroy DW. (2014) Lipid Mediators in Immune Dysfunction after Severe Inflammation. Trends in Immunology 35:12-21 
Gabrysová L., Christensen JR., Wu X., Kissenpfennig A., Malissen B., O'Garra A. (2011) Integrated T-Cell Receptor and Co-stimulatory Signals Determine TGF--Dependent Differentiation and Maintenance of Foxp3+ Regulatory T Cells. European Journal of Immunology 41(5):1242-1248

Gangnuss S., Sutton-McDowall ML., Robertson SA., Armstrong DT. (2004) Seminal Plasma Regulates Corpora Lutea Macrophage Populations during Early Pregnancy in Mice. Biology of Reproduction 71(4):1135-1141

Gao Y., Lin F., Su J., Gao Z., Li Y., Yang J., Deng Z., Liu B., Tsun A., Li B. (2012) Molecular Mechanisms Underlying the Regulation and Functional Plasticity of FOXP3+ Regulatory T Cells. Genes and Immunity 13:1-13

GarciaVelasco JA., Arici A., Zreik T., Naftolin F., Mor G. (1999) Macrophage-Derived Growth Factors Regulate FasL Expression in Endometrial Stromal Cells. Molecular Human Reproduction 5(7):642-650

Garín MI., Chu C-C., Golshayan D., Cernuda-Morollón E., Wait R., Lechler RI. (2007) Galectin-1: a Key Effector of Regulation Mediated by CD4+CD25+ T Cells. Blood 109(5):2058-2065

Genedani S., Bernardi M., Baldini MG., Bertolini A. (1992) Influence of CRH and alpha-MSH on the Migration of Human Monocytes in vitro. Neuropeptides 23(2):99-102

Genolet R., Wahli W., Michalik L. (2004) PPARs as Drug Targets to Modulate Inflammatory Responses? Current Drug Targts. Inflammation \& Allergy 3(4):361-375

Ghiringhelli F., Ménard C., Terme M., Flament C., Taieb J., Chaput N., Puig PE., Novault S., Escudier B., Vivier E., Lecesne A., Robert C., Blay JY., Bernard J., Caollat-Zucman S., Freitas A., Tursz T., Wagner-Ballon O., Capron C., Vainchencker W., Martin F., Zitvogel L. (2005a) CD4+CD25+ Regulatory T Cells Inhibit Natural Killer Cell Functions in a Transforming Growth Factor beta- Dependent Manner. Journal of Experimental Medicine 202(8):1075-1085

Ghiringhelli F., Puig PE., Roux S., Parcellier A., Schmitt E., Solary E., Kroemer G., Martin F., Chauffert B., Zitvogel L. (2005b) Tumor Cells Convert Immature Myeloid Dendritic Cells into TGF-beta-Secreting Cells Inducing CD4+CD25+ Regulatory T Cell Proliferation. Journal of Experimental Medicine 202(7):919-929

Gier HT., Marion GB. (1968) Uterus of the Cow after Parturition: Involutional Changes. American Journal of Veterinary Research 29:83-96

Gilbert RO., Shin ST., Guard CL., Erb HN., Frajblat M. (2005) Prevalence of Endometritis and its Effects on Reproductive Performance of Dairy Cows. Theriogenology 64:1879-1888

Girardi G., Yarilin D., Thurman JM., Holers VM., Salmon JE. (2006a) Complement Activation Induces Dysregulation of Angiogenic Factors and Causes Fetal Rejection and Growth Restriction. Journal of Experimental Medicine 203:2165-2175

Girardi G., Bulla R., Salmon JE., Tedesco. (2006b) The Complement System in the Pathophysiology of Pregnancy. Molecular Immunology 43:68-77

Giri SN., Emau P., CullorJS., Stabenfeldt GH., Bruss ML., Bondurant RH., Osburn BI. (1990) Effects of Endotoxin Infusion on Circulating Levels of Eicosanoids, Progesterone, Cortisol, Glucose and Lactic Acid, and Abortion in Pregnant Cows. Veterinary Microbiology 21(3):211-231

Godson C., Mitchell S., Harvey K., Petasis NA., Hogg N., Brady HR. (2000) Cutting Edge: Lipoxins Rapidly Stimulate Nonphlogistic Phagocytosis of Apoptotic Neutrophils by Monocyte-Derived Macrophages. Journal of Immunology 164(4):1663-1667

Golpon HA., Fadok VA., Taraseviciene-Stewart L., Scerbavicius R., Sauer C., Welte T., Henson PM., Voelkel NF. (2004) Life After Corpse Engulfment: Phagocytosis of Apoptotic Cells Leads to VEGF Secretion and Cell Growth. FASEB J. 18:1716 
Goñi FM and Alonso A. (2000) Membrane Fusion Induced by phospholipase C and Sphingomyelinases. Bioscience Reports 20(6):443-463

Gonthier C., Mustafa AF., Berthiaume R., Petit HV., Martineau R., Ouellet DR. (2004) Effects of Feeding Micronized and Extruded Flaxseed on Ruminal Fermentation and Nutrient Utilization by Dairy Cows. Journal of Dairy Science 87(6):1854-1863

Goodier MR. and Londei M. (2000) Lipopolysaccharide Stimulates the Proliferation of Human CD56 ${ }^{+} \mathrm{CD} 3{ }^{-}$NK Cells: a Regulatory Role of Monocytes and IL-10. The Journal of Immunology 165:139-147

Gorelik L and Flavell RA. (2000) Abrogation of TGFbeta Signaling in T Cells Leads to Spontaneous T Cell Differentiation and Autoimmune Disease. Immunity 12(2):171-181

Gorelik L., Fields PE., Flavell RA. (2000) Cutting Edge: TGF-beta Inhibits Th Type 2 Development Through Inhibition of GATA-3 Expression. Journal of immunology 165(9):4773-4777

Gorelik L., Constant S., Flavell RA. (2002) Mechanisms of Transforming Growth Factor beta-Induced Inhibition of T Helper Type I Differentiation. Journal of Experimental Medicine 195(11):1499-1505

Gorospe WC., Hughes FM Jr., Spangelo BL. (1992) Interleukin-6: Effects on and Production by Rat Granulosa Cells in vitro. Endocrinology 130(3):1750-1752

Gortham JD., Güler ML., Fenoglio D., Gubler U., Murphy KM. (1998) Low Dose TGF-beta Attenuates IL-12 Responsiveness in Murine Th Cells. Journal of Immunology 161(4):1664-1670

Gosset P., Charbonnier AS., Delerive P., Fontaine J., Staels B., Pestel J., Tonnell AB., Trottein F. (2001)

Peroxisome Proliferator-Activated Receptor Gamma Activators Affect the Maturation of Human Monocyte-Derived Dendritic Cells. European Journal of Immunology 31(10:2857-2865

Goto T., Herberman RB., Maluish A., Strong DM. (1983) Cyclic AMP as a Mediator of Prostaglandin E-Induced Suppression of Human Natural Killer Cell Activity. Journal of Immunology 130(3):1350-1355

Grohmann U., Orabona C., Fallarino F., Vacca C., Calcinaro F., Falorni A., Candeloro P., Belladonna ML., Bianchi R., Fioretti MC., Puccetti P. (2002) CTLA-4-Ig Regulates Tryptophan Catabolism in vivo. Nature Immunology 3(11):1097-1101

Guerin LR., Moldenhauer LM., Prins JR., Bromfield JJ., Hayball JD., Robertson SA. (2011) Seminal Fluid Regulates Accumulation of FOXP3+ Regulatory T cells in the Preimplantation Mouse Uterus Through Expanding the FOXP3+ Cell Pool and CCL19-Mediated Recruitment, Biology of Reproduction 85:397-408

Guma M., Ronacher L., Liu-Bryan R., Takai S., Karin M., Corr M. (2009) Caspase-1 Independent Activation of Interleukin-1beta in Neutrophil-Predominant Inflammation. Arthritis and Rheumatism 60(12):3642-3650

Gunstone FD. (1996) Fatty Acid and Lipid Chemistry. Blackie: London ISBN: 978-1-4613-6852-6

Gustafsson K., Ingelsten M., Berggvist L., Nyström J., Andersson B., Karisson-Parra A. (2008) Recruitment and Activation of Natural Killer cells in vitro by a Human Dendritic cell Vaccine. Caner Research 68(14):5965-5971

Graham MR. (2014) Effects of Lipopolysaccharide Induced Inflammatory Response on Early Embryo Survival in Ewes. Thesis: Davis College of Agriculture, Natural Resources and Design, West Virginia University

Gray CA., Taylor KM., Ramsey WS., Hill JR., Bazer FW., Bartol FF., Spencer TE. (2001a) Endometrial Glands are required for Preimplantation Conceptus Elongation and Survival. Biology of Reproduction 64(6):1608-1613

Gray CA., Bartol FF., Tarleton BJ., Wiley AA., Johnson GA., Bazer FW., Spencer TE. (2001b) Developmental Biology of Uterine Glands. Biology of Reproduction 65(5):1311-1323 
Gray CA., Burghardt RC., Johnson GA., Bazer FW., Spencer TE. (2002) Evidence that Absence of Endometrial Gland Secretions in Uterine Gland Knockout Ewes Compromises Conceptus Survival and Elongation. Reproduction 124:289-300

Greco LF., Neves Neto JT., Pedrico A., Ferrazza RA., Lima FS., Bisinotto RS., Martinez N., Garcia M., Ribeiro ES., Gomes GC., Shin JH., Ballou MA., Thatcher WW., Staples CR., Santos JEP. (2015) Effects of Altering the Ratio of Dietary n-6 to n-3 Fatty Acids on Performance and Inflammatory Responses to a Lipopolysaccharide Challenge in lactating Holstein Cows. Journal of Dairy Science 98:602-617

Grimble RF. (1998) Dietary Lipids and the Inflammatory Response. Proceedings of the Nutrition Society 57:535542

Grohmann U., Orabona C., Fallarino F., Vacca C., Calcinaro F., Falorni A., Candeloro P., Belladonna ML., Bianchi R., Fioretti MC., Puccetti P. (2002) CTLA-4 -Ig Regulates Tryptophan Catabolism in Vivo. Nature Immunology 3(11):1097-1101

Groux H., Bigler M., de Vries JE., Roncarolo MG. (1996) Interleukin-10 Induces a Long-Term Antigen-Specific Anergic State in Human CD4+ T Cells. Journal of Experimental Medicine 184(1):19-29

Gruys E., Toussanint MJM., Niewold TA., Koopmans SJ. (2005) Acute Phase Reaction and Acute Phase Proteins. Journal of Zhejiang University. Science 6B(11):1045-1056

Guleria I., Khosroshahi A., Ansari MJ., Habicht A., Azuma M., Yagita H., Noelle RJ., Coyle A., Mellor AL., Khoury SJ., Sayegh MH. (2005) A Critical Role for the Programmed Death Ligand 1 in Fetomaternal Tolerance. Journal of Experimental Medicine 202:231-237

Guillomot M. (1995) Cellular Interactions during Implantation in Domestic Ruminants. Journal of Reproduction and Fertility Supplement 49:39-51

Guillomot M., Michel C., Gaye P., Charlier N., Trojan J., Martal J. (1990) Cellular Localization of an Embryonic Interferon, Ovine Trophoblastin and its mRNA in Sheep Embryos during Early Pregnancy. Biology of the Cell 68(3):205-211

Guillomot M., Flechon JE., Wintenberger-Torres S. (1981) Conceptus Attachment in the Ewe: An Ultrastructural Study. Placenta 2(2):169-182

Hammon DS, Evjen IM., Dhiman TR., Goff JP., Walters JL. (2006) Neutrophil Function and Energy Status in Holstein Cows with Uterine Health Disorders. Veterinary Immunology and Immunopathology 113:21-29

Han C-S., Mathialagan N., Klemann SW., Roberts RM. (1997) Molecular Cloning of Ovine and Bovine Type I Interferon Receptor Subunits from Uteri, and Endometrial Expression of Messenger Ribonucleic

Han S-C., Kang G-J., Ko Y-J., Kang H-K., Moon S-W., Ann Y-S., Yoo E-S. (2012) Fermented Fish Oil Suppresses T Helper $1 / 2$ Cell Response in a mouse Model of Atopic Dermatitis via Generation of CD4+CD25+FOXP3+ T Cells. BMC Immunology 13:44

Hanna J., Wald O., Goldman-Wohl D., Prus D., Markel G., Gazit R., Katz G., Haimov-Kochman R., Fujii N., Yagel S., Peled A., Mandelboim O. (2003) CXCL12 Expression by Invasive Trophoblasts Induces the Specific Migration of CD16-Human Natural Killer Cells. Blood 102(5):1569-1577

Hanna J., Goldman-Wohl D., Hamani Y., Avraham I., Green field C., Natanson-Yaron S., Prus D., Cohen-Daniel L., Arnon TI., Manaster I., Gazit R., Yutkin V., Benharroch D., Porgador A., Keshet E., Yagel S., Mandelboim O. (2006) Decidual NK Cells Regulate Key Developmental Processes at the Human Fetal-Maternal Interface. Nature Medicine 12(9):1065-1074 
Hansen TR., Leaman DW., Cross JC., Mathialagan N., Bixby JA., Roberts RM. (1991) The Genes for the Trophoblast Interferons and the Related Interferon-aII Possess Distinct 5'-Promoter and 3' Flanking Sequences. The Journal of Biological Chemistry 266(5): 3060-3067

Hansen PJ., Soto P., Natzke RP. (2004) Mastitis and Fertility in Cattle-Possible Involvement of Inflammation or Immune Activation in Embryonic Mortality. American Journal of Reproductive Immunology 51:294-301

Hansen PJ. (2010) Medawar Redux-An Overview on the Use of Farm Animal Models to Elucidate Principles of Reproductive Immunology. American Journal of Reproductive Immunology 64(4):225-230

Hansen PJ., (2011a) The Immunology of Early Pregnancy in Farm Animals. Reproduction in Domestic Animals 46 (Suppl. 3) 18-30

Hansen PJ (2011b) Challenges to Fertility in Dairy Cattle: From Ovulation to the Fetal Stage of Pregnancy. Revista Brasileira de Reprodução Animal 35(2):229-238

Hara M., Kingsley CI., Niimi M., Read S., Turvey SE., Bushell AR., Morris PJ., Powrie F., Wood KJ. (2001) IL-10 is required for Regulatory T Cells to Mediate Tolerance to Alloantigens in Vivo. Journal of Immunology 166(6):3789-3796

Hardy K. and Spanos S. (2002) Growth Factor Expression and Function in the Human and Mouse Preimplantation Embryo. Journal of Endocrinology 172(2):221-236

Hari-Dass R., Shah C., Meyer DJ., Raynes JG. (2005) Serum Amyloid A Protein binds to Outer membrane Protein A of Gram-Negative Bacteria. Journal of Biological Chemistry 280(19):18562-18567

Harmon GS., Dumlao DS., NG DT., Barrett KE., Dennis EA., Dong H., Glass CK. (2010) Pharmacological Correction of a Defect in PPAR- $\gamma$ Signaling Ameliorates Disease Severity in Cftr-Deficient Mice. Nature Medicine 16(3):313-318

Hasday JD., Thompson C., Singh IS. (2014) Fever, Immunity, and Molecular Adaptations. Comprehensive Physiology 4:109-148

Haxhinasto S., Mathis D., Benoist C. (2008) The AKT-mTOR Axis Regulates de Novo Differentiation of CD4+Foxp3+ Cells. Journal of Experimental Medicine 205:565-574

He Y., Smith SK., Day KA., Clark DE., Licence DR., Charnock-Jones DS. (1999) Alternative Splicing of Vascular Growth Factor (VEGF)-R1 (FLT-1) pre-mRNA is Important for the Regulation of VEGF Activity. Molecular Endocrinology 13(4):537-545

Healy DA., Wallace FA., Miles EA., Calder PC., Newsholme P. (2000) The Effect of Low to Moderate Amounts of Dietary Fish Oil on Neutrophil Lipid Composition and Function. Lipids 35:763-769

Heath VL., Murphy EE., Crain C., Tomlinson MG., O'Garra A. (2000) TGF-beta1 Down-Regulates Th2 Development and Results in Decreased IL-4-Induced STAT6 Activation and GATA-3 Expression. European Journal of Immunology 30(9):2639-2649

Heikkinen J., Möttönen M., Komi J., Alanen A., Lassila O. (2003) Phenotypic Characterization of Human Decidual Macrophages. Clinical and Experimental Immunology 131(3):498-505

Heinrich PC., Castell JV., Andus T. (1990) Interleuki-6 and the Acute Phase Response. Biochemical Journal 265(3):621-636 
Helmer SD., Hansen PJ., Thatcher WW., Johnson JW., Bazer FW. (1989) Intrauterine Infusion of Highly Enriched Bovine Trophoblast Protein-1 Complex Exerts an Antiluteolytic Effect to Extend Corpus Luteum Lifespan in Cyclic Cattle. Journal of Reproduction and Fertility 87:89-101

Herath S., Fischer DP., Werling D., Williams EJ., Lilly ST., Dobson H., Bryant CE., Sheldon IM. (2006) Expression and Function of Toll-Like Receptor 4 in Bovine Endometrium. Endocrinology 147:562-570

Herath S., Lilly ST., Santos NR., Gilbert RO., Goetze L., Bryant CE., White JO., Cronin J., Sheldon IM. (2009a) Expression of Genes Associated with Immunity in the Endometrium of Cattle with Disparate Postpartum Uterine Disease and Fertility. Reproductive Biology and Endocrinology 7:55

Herath S., Lilly ST., Fischer DP., Williams EJ., Dobson H., Bryant CE., Sheldon IM. (2009b) Bacterial Lipopolysaccharide Induces an Endocrine Switch from Prostaglandin F2alpha to Prostaglandin E2 in Bovine Endometrium. Endocrinology 150(4):1912-1920

Hernandez JD., Nguyen JT., HE J., Wang W., Ardman B., Green JM., Fukuda M., Baum LG. (2006) Galectin-1 Binds Different CD43 Glycoforms to Cluster CD43 and Regulate T Cell Death. Journal of Immunology 177(8):5328-5336

Hesse DG., Tracey KJ., Fong Y., Manoque KR., Palladino MA Jr., Cerami A., Shires GT., Lowry SF. (1988) Cytokine Appearance in human Endotoxemia and Primate Bacteremia. Surgery, Gynecology, \& Obstetrics 166(2):147-153

Heusinkveld M., de Vos van Steenwijk PJ., Goedemans R., Ramwadhdoebe TH., Gorter A., Welters MJ., van der Burg SH. (2011) M2 Macrophages Induced by Prostaglandin E2 and IL-6 from Cervical Carcinoma are Switched to Activated M1 Cancer Cells. Journal of Immunology 187(3):1157-1165

Higgs GA., Moncada S., Vane JR. (1984) Eicosanoids in Inflammation. Annals of Clinical Research 16(5-6):287299

Hill JA., Haimovici F., Anderson DJ. (1987) Products of Activated Lymphocytes and Macrophages Inhibit Mouse Embryo Development in vitro. Journal of immunology 139(7):2250-2254

Hill J. and Gilbert R. (2008) Reduced Quality of Bovine Embryos Cultured in Media Conditioned by Exposure to an Inflamed Endometrium. Australian Veterinary Journal 86(8):312-316

Hirano T., Akira S., Taga T., Kishimoto T. (1990) Biological and Clinical Aspects of Interleukin-6. Immunology Today 11(12):443-449

Hogan JS., Smith KL., Hoblet KH., (1989) Field Survey of Clinical Mastitis in Low Somatic Cell Count Herds. Journal of Dairy Science 72:1547-1556

Holásková I., Lewis GS., Elliot M., Blemings KP., Dailey RA. (2004) Effect of Peptidoglycan-Polysaccharide Complex on Reproductive Efficiency in Sheep. American Journal Reproductive Immunology 52(3):197-203

Hori S., Carvalho TL., Demengeot J. (2002) CD25+ CD4+ Regulatory T Cells Suppress CD4+ T Cell-Mediated Pulmonary Hyperinflammation Driven by Pneumocystis Carinii in Immunodeficient Mice. European Journal of Immunology 32(5):1282-1291

Hori S., Nomura T., Sakaguchi S. (2003) Control of Regulatory T Cell Development By the Transcription Factor Foxp3. Science 299:1057-1061

Horwitz DA., Zheng SG., Gray JD. (2003) The Role of the Combination of IL-2 and TGF-beta or IL-10 in the Generation and Function of CD4+CD25+ and CD8+ Regulatory T Cell Subsets. Journal of Leukocyte Biology 74(4):471-478 
Huang S., Apasov S., Koshiba M., Sitkovsky M. (1997) Role of A2A Extracellular Adenosine Receptor-Mediated Signaling in Adenosine-Mediated Inhibition of T-Cell Activation and Expansion. Blood 90:1600-1610

Huang CT., Workman CJ., Flies D., Pan X., Marson AL., Zhou G., Hipkiss EL., Ravi S., Kowalski J., Levitsky HI., Powell JD., Pardoll DM., Drake CG., Vignali DA. (2004) Role of LAG-3 in Regulatory T Cells. Immunity 21(4):503-513

Huber S., Gagliani N., Esplugues E., O’Connor W Jr., Huber FJ., Chaudhry A., Kamanaka M., Kobayashi Y., Booth CJ., Rudensky AY., Roncarolo MG., Battaglia M., Flavell RA. (2011) Th17 Cells Express Interleukin-10 Receptor and are Controlled by Foxp3 $3^{-}$and Foxp $3^{+}$Regulatory CD4+ T Cells in an Interleukin-10-Dependent Manner. Immunity 34(4):554-565

Hughes DA., Southon S., Pinder AC. (1996) (n-3) Polyunsaturated Fatty Acids Modulate the Expression of Functionally Associated Molecules on Human Monocytes in Vitro. Journal of Nutrition 126(3):603-610

Hughes GC., Clark EA., Wong AH. (2013) The Intracellular Progesterone Receptor Regulates CD4+ T Cells and T Cell-dependent Antibody Responses. Journal of Leukocyte Biology 93:369-375

Hunt JS. (1989) Cytokine Networks in the Uteroplacental Unit: Macrophages as Pivotal Regulatory Cells. Journal of Reproductive Immunology 16:1-17

Hunt JS., Soares MJ., Lei MG., Smith RN., Wheaton D., Atherton RA., Morrison DC. (1989) Products of Lipopolysaccharide-Activated Macrophages (Tumor Necrosis Factor-Alpha, Transforming Growth Factor-Beta) but Not Lipopolysaccharide Modify DNA Synthesis by Rat Trophoblast Cells Exhibiting the 80-kDa Lipopolysaccharide-Binding Protein. Journal of Immunology 143(5):1606-1613

Hunt JS. (1990) Current Topic: The Role of Macrophages in the Uterine Response to Pregnancy. Placenta 11(6):467-475

Hunt JS., Petroff MG., McIntire RH., Ober C. (2005) HLA-G and Immune Tolerance in Pregnancy. FASEB Journal 19(7):681-693

Ilarregui JM., Croci DO., Bianco GA., Toscano MA., Salatino M., Vermeulen ME., Geffner JR., Rabinovich GA. (2009) Tolerogenic Signals Delivered by Dendritic Cells to T Cells Through a Galectin-1-Driven Immunoregulatory Circuit Involving Interleukin 27 and Interleukin 10. Nature immunology 10(9):981-991

Imura H., Fukata J., Mori T. (1991) Cytokines and Endocrine Functions: An Interaction between the Immune and Neuroendocrine Systems. Clinical Endocrinology 35(2):107-115

Inskeep EK. and Dailey RA. (2005) Embryonic Death in Cattle. Veterinary Clinics of North America: Food Animal Practice 21:437-461

Ito S., Ansari P., Sakatsume M., Dickensheets H., Vazquez N., Donnelly RP., Larner AC., Finbloom DS. (1999) Interleukin-10 Inhibits Expression of both Interferon-alpha- and Interferon gamma- induced Genes by Suppressing Tyrosine Phosphorylation of STAT1. Blood 93(5):1456-1463

Jackson SM., Parhami F., Xi XP., Berliner JA., Hsueh WA., Law RE., Demer LL.(1999) Peroxisome ProliferatorActivated Receptor Activators Target Human Endothelial Cells to Inhibit Leukocyte-Endothelial Cell Interaction. Arteriosclerosis, Thrombosis, and Vascular Biology 19(9):2094-2104

Jacobs AL., Sahgal PB., Julian J., Carson DD. (1992) Secretion and Hormonal Regulation of Interleukin-6 Production by Mouse Uterine Stromal and Polaried Epithelial Cells Cultured in vitro. Endocrinology 131(3):10371046 
Jacobs JR., Sommers KN., Zajac AM., Notter DR., Bowdridge SA. (2016) Early IL-4 Gene Expresion in Abomasum is associated with Resistance to Haemonchus contortus in Hair and Wool Sheep Breeds. Parasite Immunology 38:333-339

Jaiswal YK., Chaturvedi MM., Deb K. (2006) Effect of Bacterial Endotoxins on Superovulated Mouse Embryos in vivo: is CSF-1 Involved in Endotoxin-Induced Pregnancy Loss? Infectious Diseases in Obstetrics and Gynecology 2006:32050

Jaiswal WK., Agrawal V., Jaiswal YK. (2013) Lipopolysaccharide Drives Alteration of Heat Shock Proteins and Induces Failure of Blastocyst Implantation in Mouse. Biology of Reproduction 88(6):162

Jasper MJ., Care AS., Sullivan B., Ingman WV., Aplin JD., Robertson SA. (2011) Macrophage-Derived LIF and IL1B Regulate Alpha $(1,2)$ Fucosyltransferase 2 (Fut2) Expression in Mouse Uterine Epithelial Cells during Early Pregnancy. Biology of Reproduction 84:179-188

Jaudszus A., Gruen M., Watzl B., Ness C., Roth A., Lochner A., Barz D., Gabriel H., Rothe M., Jahreis G. (2013) Evaluation of Suppressive and Pro-Resolving Effects of EPA and DHA in Human Primary Monocytes and T-Helper Cells. Journal of Lipid Research 54(4):923-935

Jeschke U., Richter DU., Hammer A., Briese V., Friese K., Karsten U. (2002) Expression of the ThomsenFriedenreich Antigen and of its Putative Carrier Protein Mucin 1 in the Human Placenta and in Trophoblast Cells in Vitro. Histochemistry and Cell Biology 117(3):219-226

Jiang C., Ting AT., Seed B. (1998) PPAR- $\gamma$ Agonists Inhibit Production of Monocyte Inflammatory Cytokines. Nature 391(6662):82-86

Jin LP., Zhouu YH., Wang MY., Zhu XY., Li DJ. (2005) Blockade of CD80 and CD86 at the Time of Implantation Inhibits Maternal Rejection to the Allogeneic Fetus in Abortion-Prone Matings. Journal of Reproductive Immunology 65(2):133-146

Joachim R., Zenclussen AC., Polgar B., Douglas AJ., Fest S., Knacksted M., Klapp BF., Arck PC. (2003) The Progesterone Derivative Dydrogesterone Abrogates Murine Stress-Triggered Abortion by Inducing a Th2 Biased Local Immune Response. Steroids 68(10-13):931-940

Jones LA., Anthony JP., Henriquez FL., Lyons RE., Nickdel MB., Carter KC., Alexander J., Roberts CW. (2008) Toll-Like Receptor-4-Mediated Macrophage Activation is Differentially Regulated by Progesterone via the Glucocorticoid and Progesterone Receptors. Immunology 125:59-69

Jones ML., Mark PJ., Mori TA., Keelan JA., Waddell BJ. (2013a) Maternal Dietary Omega-3 Fatty Acid Supplementation Reduces Placental Oxidative Stress and Increases Fetal and Placental Growth in the Rat. Biology of Reproduction 88(2):37

Jones ML., Mark PJ., Keelan JA., Barden A., Mas E., Mori TA., Waddell BJ. (2013b) Maternal Dietary Omega-3 Fatty Acid Intake Increases Resolvin and Protectin Levels in the Rat Placenta. Journal of Lipid Research 54(8):2247-2254

Joosten LAB., Netea MG., Fantuzzi G., Koenders MI., Helsen MMA., Sparrer H., Pham CT., van der Meer JWM., Dinarello CA., van den Berg WB. (2009) Inflammatory Arthritis in Caspase-1 Gene Deficient Mice: Contribution of Proteinase 3 for Caspase-1 Independent Production of Bioactive IL-1 $\beta$. Arthritis and Rheumatism 60(12):3651-3662

Joshi PC., Zhou X., Cuchens M., Jones Q. (2001) Prostaglandin E2 Suppressed IL-15-Mediated Human NK Cell Function Through Down-Regulation of Common gamma-chain. Journal of Immunology 166(2):885-891

Jue DM., Shim BS., Kang YS. (1983) Inhibition of Prostaglandin Synthase Activity of Sheep Seminal Vesicular Gland by Human Serum Haptoglobin. Molecular and Cellular Biochemistry 51(2):141-147 
Kahlenberg JM. and Dubyak GR. (2004) Mechanisms of Caspase-1 Activation by P2X7 Receptor-Mediated K+ Release. American Journal of Physiology. Cell Physiology 286(5):C1100-1108

Kahlenberg JM., Lundberg KC., Kertesy SB., Qu Y., Dubyak GR. (2005) Potentiation of Caspase-1 Activation by the P2X7 Receptor is Dependent on TLR Signals and Requires NF-kappaB-Driven Protein Synthesis. Journal of Immunology 175(11):7611-7622

Kaliński P. (2012) Regulation of Immune Responses by Prostaglandin E2. Journal of Immunology 188:21-28

Kaliński P., Hilkens CM., Snijders A., Snijdewint FG., Kapsenberg ML. (1997) IL-12-Deficient Dendritic Cells, Generated in the Presence of Prostaglandin E2, Promote Type 2 Cytokine Production in Maturing Human Naive T Helper Cells. Journal of Immunology 159:28-35

Kaliński P., Schuitemaker JH., Hilkens CM., Kapsenberg ML. (1998) Prostaglandin E2 Induces the Final Maturation of IL-12-Deficient CD1a+CD83+ Dendritic Cells: the Levels of IL-12 are determined during the Final Dendritic Cell Maturation and are Resistant to Further Modulation. Journal of Immunology 161(6):2804-2809

Kaliński P., Hilkens CM., Wierenga EA., Kapsenberg ML. (1999) T-cell Priming by type-1 and type-2 Polarized Dendritic Cells: the Concept of a Third Signal. Immunology Today 20(12):561-567

Kalkunte SS., Mselle TF., Norris WE., Wira CR., Sentman CL., Sharma S. (2009) Vascular Endothelial Growth Factor C Facilitates Immune Tolerance and Endovascular Activity of Human Uterine NK Cells at the MaternalFetal Interface. Journal of Immunology 182(7):4085-4092

Kallikourdis M. and Betz AG. (2007) Periodic Accumulation of Regulatory T Cells in the Uterus: Preparation for the Implantation of a Semiallogeneic fetus? PLOS One 2(4):e382

Kallikourdis M., Andersen KG., Welch KA., Betz AG. (2007) Alloantigen-Enhances Accumulation of CCR5+ 'Effector' Regulatory T Cells in the Gravid Uterus. Proceedings of the National Academy of Sciences of the United States of America 104(2):594-599

Kahn DA. and Baltimore D. (2010) Pregnancy Induces a Fetal Antigen-Specific Maternal T Regulatory Cell Response that Contributes to Tolerance. Proceedings of the National Academy of Sciences of the United States of America 170(20):9299-9304

Kämmerer U., Eggert AO., Kapp M., McLellan AD., Geijtenbeek TB., Dietl J., van Kooyk Y., Kämpgen E. (2003) Unique Appearance of Proliferating Antigen-Presenting Cells Expressing DC-SIGN(CD209) in the Decidua of Early Human Pregnancy. American Journal of Pathology 162(3):887-896

Kanneganti TD., Ozören N., Body-Malapel M., Amer A., Park JH., Franchi L., Whitfield J., Barchet W., Colonna M., Vandenabeele P., Bertin J., Coyle A., Grant EP., Akira S., Núñez G. (2006) Bacterial RNA and Small Antiviral Compounds Activate Caspase-1 Through Cryopyrin/Nalp3. Nature 440(7081):233-236

Karin M. and Ben-Neriah Y. (2000) Phosphorylation Meets Ubiquitation: The Control of NF-kB Activity. Annual Review of Immunology 18:621-663

Karin M. and Delhase M. (2000) The I Kappa B Kinase (IKK) and NF-kappa B: Key Elements of Proinflammatory Signaling. Seminars in Immunology 12:85-98

Karrow NA. (2006) Activation of the Hypothalamic-Pituitary-Adrenal Axis and Autonomic Nervous System During Inflammation and Altered Programming of the Neuroendocrine-Immune Axis During Fetal and Neonatal Development: Lessons Learned from the Model Inflammagen, Lipopolysaccharide. Brain Behavior and Immunity 20(2):144-158

Kasama T., Miwa Y., Isozaki T., Odai T., Adachi M., Kunkel SL. (2005) Neutrophil-Derived Cytokines: Potential Therapeutic Targets in Inflammation. Current Drug Targets. Inflammation and Allergy 4(3):273-279 
Kavelaars A., Ballieux RE., Heijnen CJ. (1989) The Role of IL-1 in the Corticotropin-Releasing Factor and Arginine-Vasopressin-Induced Secretion of Immunoreactive B-Endorphin by Human Peripheral Blood Mononuclear Cells. Journal of Immunology 142(7):2338-2342

Kendall RL. And Thomas KA. (1993) Inhibition of Vascular Endothelial Cell Growth Factor Activity by an Endogenously Encoded Soluble Receptor. Proceedings of the National Academy of Sciences of the United States of America 90(22):10705-10709

Khalfoun B., Thibault F., Watier H., Bardos P., Lebranchu Y. (1997) Docosahexaenoic and Eicosapentaenoic Acids Inhibit in Vitro Human Endothelial Cell Production of Interleukin-6. Advances in Experimental Medicine and Biology 400B:589-597

Kahyrullina T., Yen JH., Jing H., Ganea D. (2008) In vitro Differentiation of Dendritic Cells in the Presence of Prostaglandin E2 Alters the IL-12/IL-23 Balance and Promotes Differentiation of Th17 Cells. Journal of Imunology 181:721-735

Kim S., Choi Y., Spencer TE., Bazer FW. (2003) Effects of the Estrous Cycle, Pregnancy and Interferon Tau on Expression of Cyclooxygenase Two (COX-2) in Ovine Endometrium. Reproductive Biology and Endocrinology 20:1-58

Kimura K., Goff JP., Kehrli ME Jr. (1999) Effects of the presence of the Mammary Gland on Expression of Neutrophil Adhesion Molecules and Myeloperoxidase Activity in Periparturient Dairy Cows. Journal of Dairy Science 82(11):2385-2392

Kimura K., Reinhardt TA., Goff JP. (2006) Parturition and hypocalcemia blunts Calcium Signals in Immune Cells of Dairy Cattle. Journal of Dairy Science 89(7):2588-2595

King AE., Critchley HO., Kelly RW. (2003) Innate Immune Defenses in the Human Endometrium. Reproductive Biology and Endocrinology 1:116

Kirshnamoorthy S., Recchiuti A., Chiang N., Fredman G., Serhan CN. (2012) Resolvin D1 Receptor Stereoselectivity and Regulation of Inflammation and Proresolving MicroRNAs. The American Journal of Pathology 180(5):2018-2027

Kluciñski W., Dembele K., Kleczkowski M., Sitarska E., Winnicka A., Sikora J. (1995) Evaluation of the effect of Experimental Cow Endometritis on Bactercidal Capability of Phagocytizing Cells Isolated from the Blood and Uterine Lumen. Zentralbl Veterinarmed A 42(7):461-466

Kluger MJ. (1978) The Evolution and Adaptive Value of Fever. American Scientist (66):38-43

Koenen HJ., Smeets RL., Vink PM., van Rijssen E., Boots AM., Joosten I. (2008) Human CD25highFoxp3pos Regulatory T Cells Differentiate into IL-17 Producing Cells. Blood 112(6):2340-2352

Koets A., Santema W., Mertens H., Oostenrijk D., Keestra M., Overdijk M., Labouriau R., Franken P., Frijters A., Nielen M., Rutten V. (2010) Susceptibility to Paratuberculosis Infection in Cattle is Associated with Singel Nucleotide Polymorphisms in Toll-Like Receptor 2 which Modulate immune Responses against Mycobacterium avium Subspecies paratuberculosis. Preventive Veterinary Medicine 93:305-315

Koga K., Izumi G., Mor G., Fujii T., Osuga Y. (2014) Toll-Like receptors at the Maternal-Fetal Interface in Normal Pregnancy and Pregnancy Complications. American Journal of Reproductive Immunology 72:192-205

Kolenko V., Rayman P., Roy B., Cathcart MK., O’Shea J., Tubbs R., Rybicki L., Bukowski R., Bukowski R., Finke J. (1999) Downregulation of JAK3 Protein Levels in T Lymphocytes by Prostaglandin E2 and other Cyclic Adenosine Monophosphate-Elevating Agents: Impact on Interleukin-2 Receptor Signaling Pathway. Blood 93(7):2308-2318 
Konsman JP., Parnet P., Dantzer R. (2002) Cytokine -Induced Sickness Behaviour: Mechanisms and Implications. Trends in Neurosciences 25(30:154-159

Konsman JP., Vigues S., Mackerlova L., Bristow A., Blomqvist A. (2004) Rat Brain Vascular Distribution of Interleukin-1 Type-1 Receptor Immunoreactivity: Relationship to Patterns of Inducible Cyclooxygenase Expression by Peripheral Inflammatory Stimuli. Journal of Comparative Neurology 472:113-129

Koopman LA., Kopcow HD., Rybalov B., Boyson JE., Orange JS., Schatz F., Masch R., Lockwood CJ., Schachter AD., Park PJ., Strominger JL. (2003) Human Decidual Natural Killer Cells are a Unique NK Cell Subset with Immunomodulatory Potential. Journal of Experimental Medicine 198(8):1201-1212

Kopcow HD., Allan DS., Chen X., Rybalov B., Andzelm MM., Ge B., Strominger JL. (2005) Human Decidual NK Cells form Immature Activating Synapses and are Not Cytotoxic. Proceedings of the National Academy of Sciences of the United States of America 102(43):15563-15568

Kopcow HD., Rosetti F., Leung Y., Allan DAJ., Kutok JL., Strominger JL. (2008) T Cell Apoptosis at the MaternalFetal Interface in Early Human Pregnancy, involvement of Galectin-1. PNAS 105(47):18472-18477

Koppelmann B., Neefjes JJ., de Vries JE., de Wall Malefyt R. (1997) Interleukin-10 Downregulates MHC Class II ab Peptide Complexes at the Plasma Membrane of Monocytes by Affecting Arrival and Recycling. Immunity 7(6):861-871

Koshida H. and Kotake Y. (1994) Corticotropin-Releasing Hormone Enhances the Superoxide Anion Production of Rabbit Peritoneal Macrophages Stimulated with N-formyl-methionyl-leucyl-phenylalanine. Life Sciences 54(8):539543

Kozma N., Halasz M., Polgar B., Poehlmann TG., Markert UR., Palkovics T., Keszei M., Par G., Kiss K., Szeberenyi J., Grama L., Szekeres-Bartho J. (2006) PIBF Activates STAT6 via Binding to a Novel IL-4 Receptor. Journal of Immunology 176(2):819-826

Kraut N., Snider L., Chen CM., Tapscott SJ., Groudine M. (1998) Requirement of the Mouse I-mfa Gene for Placental Development and Skeletal Patterning. EMBO J 17(21):6276-6288

Krishnamoorthy S., Recchiuti A., Chiang N., Yacoubian S., Lee CH., Yang R., Petasis NA., Serhan CN. (2010) Resolvin D1 Binds Human Phagocytes with Evidence for Proresolving Receptors. Proceedings of the National Academy of Sciences of the United States of America 107(4):1660-1665

Kubes P., Suzuki M., Granger DN. (1991) Nitric Oxide: AN Endogenous Modulator of Leukocyte Adhesion. Proceedings of the National Academy of Science of the United States of America 88(11):4651-4655

Kumar N., Gupta G., Anilkumar K., Fatima N., Karnati R., Reddy GV., Giri PV., Reddanna P. (2016) 15Lipoxygenase Metabolites of $\alpha$-Linolenic Acid, [13-(S)-HPOTrE and 13-(S)-HOTrE], Mediate Anti-Inflammatory Effects by Inactivating NLRP3 Inflammasome. Scientific Reports 6:31649

Kunkel SL., Spengler M., May MA., Spengler R., Larrick J., Remick D. (1988) Prostaglandin E2 Regulates Macrophage-Derived Tumor Necrosis Factor Gene Expression. Journal of Biological Chemistry 263(11):5380-5384

Küther K., Audigé L., Kube P., Welle M. (1998) Bovine Mast Cells: Distribution, Density, Heterogeneity, and influence of Fixation Techniques. Cell and Tissue Research 293:111-119

Kuwamura H., Aswad F., Minagawa M., Govindarajan S., Dennert G. (2006) P2X7 Receptors Regulate NKT Cells in Autoimmune Hepatitis. Nature 176(4):2152-2160

Kwak-Kim J., Bao S., Lee SK., Kim JW., Gilman-Sachs A. (2014) Immunological Modes of Pregnancy Loss: Inflammation, immune Effectors, and Stress. American Journal of Reproductive Immunology 72:129-140 
La Rocca C., Carbone F., Longobardi S., Matarese G. (2014) The Immunology of Pregnancy: Regulatory T Cells Control Maternal Immune Tolerance Toward the Fetus. Immunology Letters 162:41-48

Lam C., Lim K-H., Karumanchi SA. (2005) Circulating Angiogenic Factors in the Pathogenesis and Prediction of Preeclampsia. Hypertension 46(5):1077-1085

Lappas M., Permezel M., Georgiou HM., Rice GE. (2002) Regulation of Proinflammatory Cytokines in Human Gestational Tissues by Peroxisome Proliferator-Activated Receptor-gamma: Effect of 15-deoxy-Delta $(12,14)$ PGJ(2) and Troglitazone. Journal of Clinical Endocrinology and Metabolism 87(10):4667-4672

Lazarus M., Yoshida K., Coppari R., Bass CE., Mochizuki T., Lowell BB., Saper CB. (2007) EP3 Prostaglandin Receptors in the Median Preoptic Nucleus are Critical for Fever Responses. Nature Neuroscience 10(9):1131-1133

Lea RG., Sandra O. (2007) Immunoendocrine Aspects of Endometrial Function and Implantation. Reproduction 134:389-404

LeBlanc SJ. (2008) Postpartum Uterine Disease and Dairy Herd Reproductive Performance: A Review. The Veterinary Journal 176:102-114

Lecchi C., Dilda F., Sartorelli P., Ceciliani F. (2012) Widespread Expression of SAA and Hp RNA in Bovine Tissues after Evaluation of Suitable Reference Genes. Veterinary Immunology and Immunopathology 145(1-2):556562

Lee JH., Ulrich B., Cho J., Park J., Kim CH. (2011) Progesterone Promotes Differentiation of Human Cord Blood Fetal T Cells into T Regulatory Cells but Suppresses their Differentiation into Th17 Cells. Journal of Immunology 187(4):1778-1787

Lee JH., Lydon JP., Kim CH. (2012) Progesterone Suppresses the mTOR Pathway and Promotes Generation of Induced Regulatory T cells with increased Stability. European Journal of Immunology 42:2683-2696

Lee TH., Horton CE., Kyan-Aung U., Haskard D., Crea AE., Spur BW. (1989) Lipoxin A4 and Lipoxin B4 Inhibit Chemotactic Responses of Human Neutrophils Stimulated by Leukotriene B4 and N-formyl-L-methionyl-L-leucylL-phenylalanine. Clinical Science (London) 77(2):195-203

van der Leij J., van der Berg A., Blokzijl T., Harms G., van Goor H., Zwiers P., van Weeghel R., Poppema S., Visser L. (2004) Dimeric Galectin-1 Induces IL-10 Production in T-Lymphocytes: An Important Tool in the Regulation of the Immune Response. Journal of Pathology 204:511-518

Leslie KE., (1983) The Events of Normal and Abnormal Postpartum Endocrinology and uterine Involution in Dairy Cows: A Review. The Canadian Veterinary Journal 24:67-71

Levy BD., Clish CB., Schmidt B., Gronert K., Serhan N. (2001) Lipid Mediator Class Switching during Acute Inflammation: Signals in Resolution. Nature 27:612-619

Lewis GS. (1997) Uterine Health and Disorders. Journal of Diary Science 80:984-994

Li C-C., Yang H-T., Hou Y-C., Chiu Y-S., Chiu W-C. (2014) Dietary Fish Oil Reduces Systemic Inflammation and Ameliorates Sepsis-Induced Liver Injury by Upregulating the Peroxisome Proliferator-Activated Receptor GammaMediated Pathway in Septic Mice. Journal of Nutritional Biochemistry 25:19-25

Li Q., Liu L., Zhang Q., Liu S., Ge D., You Z. (2014) Interleukin-17 Indirectly Promotes M2 Macrophage Differentiation through Stimulation of COX-2/PGE2 Pathway in the Cancer Cells. Cancer Research and Treatment 46(3):297-306

Li Y., Dalli J., Chiang N., Baron RM., Quintana C., Serhan CN. (2013) Plasticity of Leukocytic Exudates in Resolving Acute Inflammation is regulated by MicroRNA and Proresolving Mediators. Immunity 39(5):885-898 
Li YH., Zhou WH., Tao Y., Wang SC., Jiang YL., Zhang D., Piao HL., Fu Q., Li DJ., Du MR. (2016) The Galectin9/Tim-3 pathway is Involved in the Regulation of NK Cell Function at the Maternal-Fetal Interface in Early Pregnancy. Cellular and Molecular Immunology 13:73-81

Li X., Lonard DM., O’Malley BW. (2004) A Contemporary Understanding of Progesterone Receptor Function. Mechanisms of Ageing and Development 125(10-11):669-678

Lian M., Luo W., Sui Y., Li Z., Hua J. (2015) Dietary n-3 PUFA Protects Mice from Con A Induced Liver Injury by Modulating Regulatory T Cells and PPAR- $\gamma$ Expression. PLOS One 10(7):e0132741

Liang B., Workman C., Lee J., Chew C., Dale BM., Colonna L., Flores M., Li N., Schweighoffer E., Greenberg S., Tybulewicz V., Vignali D., Clynes R. (2008) Regulatory T Cells Inhibit Dendritic Cells by Lymphocyte Activation Gene-3 Engagement of MHC Class II. Journal of Immunology 180(9):5916-5926

Liao Z., Dong J., Wu W., Yang T., Wang T., Guo L., Chen L., Xu D., Wen F. (2012) Resolvin D1 Attenuates Inflammation in Lipopolysaccharide-Induced Acute Lung Injury through a Process Involving the PPAR $\gamma / \mathrm{NF}-\mathrm{kB}$ Pathway. Respiratory Research 13:110

Lim H., Paria BC., Das SK., Dinchuk JE., Langenbach R., Trzaskos JM., Dey SK. (1997) Multiple Female Reproductive Failures in Cyclooxygenase 2-Deficient Mice. Cell 91:197-208

Lim H., Gupta RA., Ma WG., Paria BC., Moller DE., Morrow JD., DuBois RN., Trzaskos JM., Dey SK.(1999) Cyclooxygenase-2 Derived Prostacyclin Mediates Embryo Implantation in the Mouse via PPAR delta. Genes \& Development 13(12):1561-1574

Lin JT., Martin SL., Xia L., Gorham JD. (2005) TGF-beta 1 Uses Distinct Mechanisms to Inhibit IFN-gamma Expressionin CD4+ T Cells at Priming and at Recall: Differential Involvement of Stat4 and T-bet. Journal of Immunology 174(10):5950-5958

Lissauer DM., Piper KP., Moss PA., Kilby MD. (2009) Fetal Microchimerism: the Cellular and Immunological Legacy of Pregnancy. Expert Reviews in Molecular Medicine 11:e33

Lissauer DM., Eldershaw SA., Inman CF., Coomarasamy A., Moss PAH., Kilby MD. (2015) Progesterone Promotes Maternal-Fetal Tolerance by Reducing Human Maternal T-cell Polyfunctionality and Inducing a Specific Cytokine Profile. European Journal of Immunology 45:2858-2872

Liu WJ., Peltier MR., Hansen PJ. (1999) Binding of Ovine Uterine Serpin to Lymphocytes. American Journal of Immunology 41(6):428-432

Livak KJ. And Schmittgen. (2001) Analysis of Relative Gene Expresion Data Using Real-Time Quantitative PCR and the $2^{-\Delta \Delta \mathrm{CT}}$ Method. Methods $402-408$

Lo CJ., Chiu KC., Fu M., Lo R., Helton S. (1999) Fish Oil Decreases Tumor Necrosis Factor Gene Transcription by Altering the NF Kappa B Activity. Journal of Surgical Research 82(2):216-212

Lohr J., Knoechel B., Abbas AK., (2006) Regulatory T Cells in the Periphery. Immunological Reviews 212:149-162

Loureiro B., Brad AM., Hansen PJ. (2007) Heat Shock and Tumor Necrosis Factor-alpha Induce Apoptosis in Bovine Preimplantation Embryos through a Caspase-9 -dependent Mechanisms. Reproduction 133(6):1129-1137

Lu L., Li W., Fu F., Chambers FG., Qian S., Fung JJ., Thomson AW. (1997) Blockade of the CD40-CD40 Ligand Pathway Potentiates the Capacity of Donor-Derived Dendritic Cell Progenitors to Induce Long-Term Cardiac Allograft Survival. Transplantation 64(12):1808-1815

Luft T., Jefford M., Luetijens P., Toy T., Hochrein H., Masterman KA., Maliszewski C., Shortman K., Cebon J., Maraskovsky E. (2002) Functionally Distinct Dendritic Cell (DC) Populations Induced by Physiologic Stimuli: Prostaglandin E(2) Regulates the Migratory Capacity of Specific DC Subsets. Blood 100(4):1362-1372 
Lumpkin MD. (1987) The Regulation of ACTH Secretion by IL-1. Science 238(4826):452-454

Madden KS., Sanders VM., Felten DL. (1995) Catecholamine Influences and Sympathetic Neural Modulation of Immune Responsiveness. Annual Review of Pharmacology and Toxicology 35:417-448

Maddox JF. and Serhan CN. (1996) Lipoxin A4 and B4 are Potent Stimuli for Human Monocyte Migration and Adhesion: Selective Inactivation by Dehydrogenation and Reduction. The Journal of Experimental Medicine $183: 137-146$

Magiakou MA., Mastorakos G., Webster E., Chrousos GP. (1997) The Hypothalamic-Pituitary-Adrenal Axis and the Female Reproductive System. Annals of the New York Academy of Sciences 816:42-56

Mahnke K., Johnson TS., Ring S., Enk AH. (2007) Tolerogenic Dendritic Cells and Regulatory T Cells: A TwoWay Relationship. Journal of Dermatological Science 46(3):159-167

Maia MR., Chaudhary LC., Figueres L., Wallace RJ. (2007) Metabolism of Polyunsaturated Fatty Acids and their Toxcity to the Microflora of the Rumen. Antonie van Leeuwenhoek 91(4):303-314

Mailliard RB., Alber SM., Shen H., Watkins SC., Kirkwood JM., Herberman RB., Kalinski P. (2005) IL-18-Induced CD83+CCR7+ NK Helper Cells. Journal of Experimental Medicine 202(7):941-953

Majewski AC., Tekin S., Hansen PJ. (2001) Local versus Systemic Control of Numbers of Endometrial T-cells During Pregnancy in Sheep. Immunology 102(3):317-322

Makrigiannakis A., Margioris AN., Le Goascogne C., Zoumakis E., Nikas G.,Stournaras C., Psychoyos A., Gravanis A. (1995) Corticotropin-Releasing Hormone (CRH) is Expressed at the Implantation Sites of Early Pregnant Rat Uterus. Life Sciences 57(20):1869-1875

Makrigiannakis A., Margioris AN., Zoumakis E., Stournaras C., Gravanis A. (1999) The Transcription of Corticotropin-Releasing Hormone in Human Endometrial Cells is regulated by Cytokines. Neuroendocrinology 70(6):451-459

Mallard BA., Dekkers JC., Ireland MJ., Leslie KE., Sharif S., Vankampen CL., Wagter L., Wilkie BN. (1998) Alteration in Immune Responsiveness during the Peripartum Period and its Ramification on Dairy Cow and Calf health. Journal of Dairy Science 81:585-595

Mao D., Wu X., Deppong C., Friend LD,. Dolecki G., Nelson DM., Molina H. (2003) Negligible Role of Antibodies and C5 in Pregnancy Loss Associated Exclusively with C3-Dependent Mechanisms through Complement Alternative Pathway. Immunity 19:813-822

Mao G., Wang J.,Kang Y., Tai P., Wen J., Zou Q., Li G., Ouyang H., Xia G., Want G. (2010) Progesterone Increases Systemic and Local Uterine Proportions of CD4+CD25+ Treg Cells During Midterm Pregnancy in Mice. Endocrinology 151:5477-5488

Maquoi E., van den Brûle FA., Castronovo V., Foidart JM. (1997) Changes in the Distribution Pattern of Galectin-1 and Galectin-3 in Human Placenta Correlates with the Differentiation Pathways of Trophoblasts. Placenta 18(56):433-439

Marei WF., Wathes DC., Fouladi-Nashta AA. (2009) The Effect of Linolenic Acid on Bovine Oocyte Maturation and Development. Biology of Reproduction 81:1064-1072

Mariathasan S., Weiss DS., Newton K., McBride J., O’Rourke K., Roose-Girma M., Lee WP., Weinrauch Y., Monack DM., Dixit VM. (2006) Cryopyrin Activates the Inflammasome in Response to Toxins and ATP. Nature 440(9):228-232

Martal JL., Chene NM., Huynh LP., Haridon RML., Reinaud PB., Guillomot MW., Charlier MA., Charpigny SY. (1998) IFN-tau: A Novel Subtype I IFN1. Structural Characteristics, Non-Ubiquitous Expression, Structure- 
Function Relationships, a Pregnancy Hormonal Embryonic Signal and Cross-Species Therapeutic Potentialities. Biochimie 80:755-777

Martinon F., Agostini L., Maylan E., Tschopp J. (2004) Identification of Bacterial Muramyl Dipeptide as Activator of the NALP3/Cryopyrin Inflammasome. Current Biology 14(21):1929-1934

Martinon F., Pétrilli V., Mayor A., Tardivel A., Tschopp J. (2006) Gout-Associated Uric Acid Crystals Activate the NALP3 Inflammasome. Nature 440(7081):237-241

Martinon F., Mayor A., Tschopp J. (2009) The Inflammasomes: Guardians of the Body. Annual Review of Immunology 27:229-265

Mas E., Croft KD., Zahra P., Barden A., Mori TA. (2012) Resolvins D1, D2, and Other Mediators of Self-Limited Resolution of Inflammation in Human Blood following n-3 Fatty Acid Supplementation. Clinical Chemistry 58(10):1476-1484

Mastorakos G., Webster EL., Friedman TC., Chrousos GP. (1993) Immunoreactive Corticotropin-Releasing Hormone and its Binding Sites in the Rat Ovary. Journal of Clinical Investigation 92(2):961-968

Mattos R., Staples CR., Williams J., Amorocho A., McGuire MA., Thatcher WW. (2002) Uterine, Ovarian, and Production Responses of Lactating Dairy Cows to Increasing Dietary Concentrations of Menhaden Fish Meal. Journal of Dairy Science 85(4):755-764

Mattos R., Guzeloglu A., Badinga L., Staples CR., Thatcher WW. (2003) Polyunsaturated Fatty Acids and Bovine Interferon- $\tau$ Modify Phorbol Ester-Induced Secretion of Prostaglandin $F_{2 \alpha}$ and Expression of Prostaglandin Endoperoxide Synthase-2 and Phospholipase-A2 in Bovine Endometrial Cells. Biology of Reproduction 69:780-787

Mattos R., Staples CR., Arteche A., Wiltbank MC., Diaz FJ., Jenkins TC., Thatcher WW. (2004) The Effects of Feeding Fish Oil on Uterine Secretion of $\mathrm{PGF}_{2 \alpha}$, Milk Composition, and Metabolic Status of Periparturient Holstein Cows. Journal of Dairy Science 87:921-932

Maynard CL. And Weaver CT. (2008) Diversity in the Contribution of Interleukin-10 to T-Cell Mediated Immune Regulation. Immunological Reviews 226:219-233

McCann SM., Lyson K., Karanth S., Gimeno M., Belova N., Kamat A., Rettori V. (1995) Mechanism of Action of Cytokines to Induce the Pattern of Pituitary Hormone Secretion in Infection. Annals of the New York Academy of Sciences 771:386-395

McCartney-Francis N., Jin W., Wahl SM. (2004) Aberrant Toll Like Receptor Expression and Endotoxin Hypersensitivity in Mice Lacking a Functional TGF beta 1 Signaling Pathway. Journal of Immunology 172(6):38143821

McGillis JP., Park A., RubinFletter P., Turck C., Dallman MF., Payan DG. (1989) Stimulation of Rat BLymphocyte Proliferation by Corticotropin-Releasing Factor. Journal of Neuroscience Research 23(30:346-352

McIlroy A., Caron G., Blanchard S., Frémaux I., Duluc D., Delneste Y., chevailler A., Jeannin P. (2006) Histamine and Prostaglandin E up-regulate the Production of Th2-Attracting Chemokines (CCL17 and CCL22) and Downregulate IFN-gamma-Induced CXCL10 Production by Immature Human Dendritic Cells. Immunology 117(4):507-516

McMaster MT., Newton RC., Dey SK., Andrews GK. (1992) Activation and Distribution of Inflammatory Cells in the Mouse Uterus during the Preimplantation Period. Journal of Immunology 148(6):1699-1705

Medzhitov R. (2008) Origin and Physiological Roles of Inflammation. Nature 454:428-435

Mei s., Tan J., Chen H., Chen Y., Zhang J. (2010) Changes of CD4+CD25 high Regulatory T Cells and FOXP3 Expression in Unexplained Recurrent Spontaneous Abortion Patients. Fertility and Sterility 94(6):2244-2247 
Mellor AL., Sivakumar J., Chandler P., Smith K., Molina H., Mao D., Munn DH. (2001) Prevention of T CellDriven Complement Activation and Inflammation by Tryptophan Catabolism during Pregnancy. Nature Immunology 2:64-68

Mesgaran DM., Jafarpoor JR., Mesgaran DS. (2012) Milk Production, Milk Fatty Acid Composition, and Blood Biochemical Parameters of Holstein Dairy Cows Fed Whole or Ground Flaxseed instead of Extruded Soybeans in the First Half of Lactation. Iranian Journal of Veterinary Research 13(3):203-209

Meylan E., Tschopp J., Karin M. (2006) Intracellular Pattern Recognition Receptors in the Host Response. Nature 442:39-44

Michael AE., Thurston LM., Rae MT. (2003) Glucocorticoid Metabolism and Reproduction: A Tale of Two Enzymes. Reproduction 126:425-441

Miles EA. and Calder PC. (1998) Modulation of Immune Function by Dietary Fatty Acids. Proceedings of the Nutritional Society 57:277-292

Miller L., Alley EW., Murphy Wj., Russell SW., Hunt JS. (1996) Progesterone Inhibits Inducible Nitric Oxide Synthase Gene Expression and Nitric Oxide Production in Murine Macrophages. Journal of Leukocyte Biology 59(3):442-450

Miranda D., Puente J., Blanco L., Wolf ME., Mosnaim AD. (1998) In Vitro Effect of Bacterial Lipopolysaccharide on the Cytotoxicity of Human Natural Killer Cells. Research Communications in Molecular Pathology and Pharmacology 100:3-14

Mitchell S., Thomas G., Harvey K., Cottell D., Reville K., Berlasconi G., Petasis NA., Erwig L., Rees AJ., Savill J., Brady HR., Godson C. (2002) Lipoxins, Aspirin-Triggered Epi-Lipoxins, Lipoxin Stable Analogues, and the Resolution of inflammation: Stimulation of Macrophage Phagocytosis of Apoptotic Neutrophils in Vivo. Journal of the American Society of Nephrology 13(10):2497-2507

Miyaura H. and Iwata M. (2002) Direct and Indirect Inhibition of Th1 development by Progesterone and Glucocorticoids. Journal of Immunology 168(3):1087-1094

Miyazaki S., Tsuda H., Sakai M., Hori s., Sasaki Y., Futatani T., Miyawaki T., Saito S. (2003) Predominance of TH2-Promoting Dendritic cells in Early Human Pregnancy Decidua. Journal of Leukocyte Biology 74(4):541-522

Moldenhauer LM., Diener KR., Thring DM., Brown MP., Hayball JD., Robertson SA. (2009a) Cross-Presentation of Male Seminal Fluid Antigens Elicits T Cell Activation to Initiate the Female Immune Response to Pregnancy. (2009a) Journal of Immunology 182(12):8080-8093

Moldenhauer LM., Hayball JD., Robertson SA. (2009b) Immune-Deviating Cytokines determine the Maternal T Cell Response and Conceptus Tolerance or Rejection. Biology of Reproduction 81(Suppl 1):86

Moldenhauer LM., Keenihan SN., Hayball JD., Robertson SA. (2010) GM-CSF is an Essential Regulator of T Cell Activation Competence in Uterine Dendritic Cells during Early Pregnancy in Mice. Journal of Immunology 185(11):7085-7096

Molina H. (2005) Complement Regulation during Pregnancy. Immunologic Research 32:187-192

Moncada S. and Higgs A. (1993) The L-Arginine-Nitric Oxide Pathway. The New England Journal of Medicine 329:2002-2012

Moore KW., de Wall Malefyt R., Coffman RL., O'Garra A. (2001) Interleukin-10 and the Interleukin-10 Receptor. Annual Review of Immunology 19:683-765

Morelli AE., and Thomson AW. (2007) Tolergenic Dendritic Cells and the Quest for Transplant Tolerance. Nature Reviews Immunology 7(8):610-621 
Mor G. (2008) Inflammation and Pregnancy: The Role of Toll-Like Receptors in Trophoblast-Immune Interaction. Annals of the New York Academy of Sciences 1127:121-128

Mor G., Romero R., Aldo PB., Abrahams VM. (2005) Is the Trophoblast an Immune Regulator? The Role of TollLike Receptors during Pregnancy. Critical Reviews in Immunology 25(5):375-388

Morimoto K., Shirata N., Taketomi Y., Tsuchiya S., Sedqi-Nishida E., Inazumi T., Kabashima K., Tanaka S., Murakami M., Narumiya S., Sugimoto Y. (2014) Prostaglandin E2-EP3 Signaling Induces Inflammatory Swelling by Mast Cell Activation. Journal of Immunology 192(3):1130-1137

Morris DG (2014) Negative Energy Balance and Immune Function in Cows. Agriculture and Food Development Authority. Animal \& Grassland Research and Innovation, Technology Updates: project number 5929

Motran CC., Molinder KM., Liu SD., Poirier F., Miceli MC. (2008) Galectin-1 Functions as a Th2 Cytokine that Selectively Induces Th1 Apoptosis and Promotes Th2 Function. European Journal of Immunology 38(11):30153027

Mulac-Jericevic B. and Conneely OM. (2004) Reproductive Tissue Selective Actions of Progesterone Receptors. Reproduction 128(2):139-146

Munder M., Eichmann K., Modolell M. (1998) Alternative Metabolic States in Murine Macrophages Reflected by the Nitric Oxide Synthase/Arginase Balance: Competitive Regulation by CD4+ T Cells Correlates with Th1/Th2 Phenotype. Journal of Immunology 160(11):5347-5354

Munn DH., Zhou M., Attwood JT., Bondarev I., Conway SJ., Marshall B., Brown C., Mellor AL. (1998) Prevention of Allogenic Fetal Rejection of Trytophan Catabolism. Science 281:1191-1194

Munn DH., Shafizadeh E., Attwood JT., Bondarev I., Pashine A., Mellor AL. (1999) Inhibition of T cell

Proliferation by Macrophage Tryptophan Catabolism. Journal of Experimental Medicine 189(9):1363-1372

Murata H., Shimada N., Yoshioka M. (2004) Current Research on Acute Phase Proteins in Veterinary Diagnosis: An Overview. Veterinary Journal 168:28-40

Murphy MG. (1990) Dietary Fatty Acids and Membrane Protein Function. Journal of Nutritional Biochemistry 1(2):68-79

Murphy K. (2012) Janeway's Immunobiology $8^{\text {th }}$ Edition Garland Science, Taylor \& Francis Group, LLC

Murphy SP., Fast LD., Hanna NN., Sharma S. (2005) Uterine NK Cells Mediate Inflammation-Induced Fetal Demise in IL-10 Null Mice. The Journal of Immunology 175:4084-4090

Murphy SP., Hanna NN., Fast LD., Shaw SK., Berg G., Padbury JF., Romero R., Sharma S. (2009) Evidence for Participation of Uterine Natural Killer Cells in the Mechanisms Responsible for Spontaneous Preterm Labor and Delivery. American Journal of Obstetrics and Gynecology 200(3):308

Murugaiyan G., Mittal A., Lopez-Diego R., Maier LM., Anderson DE., Weiner HL. (2009) IL-27 is a Key Regulator of IL-10 and IL-17 Production by Human CD4+ T cells. Journal of Immunology 183(4):2435-2443

Mustafa AF., McKinnon JJ., Christensen DA., He T. (2002) Effects of Micronization of Flaxseed on Nutrient Disappearance in the Gastrointestinal Tract of Steers. Animal Feed Science and Technology 95:123-132

Muthuswamy R., Urban J., Lee JJ., Reinhart TA., Bartlett D., Kalinski P. (2008) Ability of Mature Dendritic Cells to Interact with Regulatory T Cells is imprinted during Maturation. Cancer Research 68(14):5972-5978

Muzio M., Bosisio D., Polentarutti N., D’amico G., Stoppacciaro A., Mancinelli R., van’t Veer C., Penton-Rol G., Ruco LP., Allavena P., Mantovani A. (2000) Differential Expression and Regulation of Toll-Like Receptors (TLR) in Human Leukocytes: Selective Expression of TLR3 in Dendritic Cells. Journal of Immunology 164(11):5998-6004 
Nagamatsu T. and Schust DJ. (2010) The Contribution of Macrophages to Normal and Pathological Pregnancies. American Journal of Reproductive immunology 63(6):460-471

Naitoh Y., Fukata J., Tominaga T., Nakai Y., Tamai S., Mori K., Imura H. (1988) Interelukin-6 Stimulates the Secretion of Adrenocorticotropic Hormone in Conscious, Freely-Moving Rats. Biochemical and Biophysical Research Communications 155(3):1459-1463

Nakamura K., Kitani A., Strober W. (2001) Cell Contact-Dependent Immunosuppression by CD4(+)CD25(+) Regulatory T Cells is Mediated by Cell Surface-Bound Transforming Growth Factor Beta. Journal of Experimental Medicine 194(5):626-644

Nakamura K., Kitani A., Fuss I., Pedersen A., Harada N., Nawata H., Strober W. (2004) TGF-beta 1 Plays an Important Role in the Mechanism of CD4+CD25+ Regulatory T Cell Activity in both Humans and Mice. Journal of Immunology 172(2):834-842

Nakamura H., Jasper MJ., Hull ML., Aplin JD., Robertson SA. (2012) Macrophages Regulate Expression of Alpha1,2 Fucosyltransferase Genes in Human Endometrial Epithelial Cells. Molecular Human Reproduction 18(4):204-215

Nakanishi K., Yoshimoto T., Tsutsui H., Okamura H. (2001) Interleukin-18 Regulates Both Th1 and Th2 Responses. Annual Review of Immunology 9:423-474

Nakano T., Ohara O., Teraoka H., Arita H. (1990) Glucocorticoids Suppress Group II Phospholipase A2 Production by Blocking mRNA Synthesis and Posttranscriptional Expression. Journal of Biological Chemistry 265(21):1274512748

Nakao A., Afrakhte M., Morén A., Nakayama T., Christian JL., Heuchel R., Itoh s., Kawabata M., Heldin NE., Heldin NE., Heldin CH., ten Dijke P. (1997) Identification of Smad7, a TGF beta-Inducible Antagonist of TGF-beta Signaling. Nature 389(6651):631-635

Nakashima A., Shiozaki A., Myojo S., Ito M., Tatematsu M., Sakai M., Takemori Y., Ogawa K., Nagata K., Saito S. (2008) Granulysin Produced by Uterine Natural Killer Cells Induces Apoptosis of Extravillous Trophoblasts in Spontaneous Abortion. American Journal of Pathology 173(3):653-664

Nancy P., Tagliani E., Tay CS., Asp P., Levy DE., Erlebacher A. (2012) Chemokine Gene Silencing in Decidual Stromal Cells Limits T Cell Access to the Maternal-Fetal Interface. Science 336:1317-1321

Nathan C. (2002) Points of Control in Inflammation. Nature 420(6917):846-852

Nathan C. (2006) Neutrophils and Immunity: Challenges and Opportunities. Nature Reviews. Immunology 6(3):173182

Netea MG., Kullberg BJ., Van der Meer JWM. (2000) Circulating Cytokines as Mediators of Fever. Clinical Infectious Diseases 31:S178-184

Nguyen JT., Evans DP., Galvan M., Pace KE., Leitenberg D., Bui TN., Baum LG. (2001) CD45 Modulates Galectin-1-Induced T Cell Death: Regulation by Expression of Core 2 O-Glycans. Journal of Immunology 167(10):5697-5707

Niiro H., Otsuka T., Tanabe T., Hara S., Kuga S., Nemoto Y., Tanaka Y., Nakashima H., Kitajima S., Abe M. (1995) Inhibition by Interleukin-10 of Inducible Cyclooxygenase Expression in Lipopolysaccharide-Stimulated Monocytes: its Underlying Mechanism in Comparison with Interleukin-4. Blood 85:3736-3745

Niiro H., Otsuka T., Ogami E., Yamaoka K., Nagano S., Akahoshi M., Nakashima H., Arinobu Y., Izuhara K., Niho Y. (1998) MAP Kinase Pathways as a Route for Regulatory Mechanisms of IL-10 and IL-4 which Inhibit COX-2 Expression in Human Monocytes. Biochemical and Biophysical Research Communications 250(2):200-205 
Niswender GD., Juengel JL., Silva PJ., Rollyson MK., McIntush EW. (2000) Mechanisms Controlling the Function and Life Span of the Corpus Luteum. Physiological Reviews 80:1-29

Noel A., Herman A., Johnson GA., Gray CA., Stewart MD., Bazer FW., Gertler A., Spencer TE. (2003) Ovine Placental Lactogen Specifically Binds to Endometrial Glands of the Ovine Uterus. Biology of Reproduction 68:772780

Norling LV., Sampaio AL., Cooper D., Perretti M. (2008) Inhibitory Control of Endothelial Galectin-1 on in vitro and in vivo Lymphocyte Trafficking. FASEB Journal 22(3):682-690

Norling LV., Spite M., Yang R., Flower RJ., Perretti M., Serhan CN. (2011) Cutting Edge: Humanized NanoProresolving Medicines Mimic Inflammation-Resolution and Enhance Wound Healing. Journal of Immunology 186(10):5543-5547

Norris PC., Gosselin D., Reichart D., Glass CK., Dennis EA. (2014) Phospholipase A 2 Regulates Eicosanoid Class Switching during Inflammasome Activation. Proceedings of the National Academy of Sciences of the United States of America 111(35):12746-12751

Novick D., Kim SH., Fantuzzi G., Reznikov LL., Dinarello CA., Rubinstein M. (1999) Interleukin-18 Binding Protein: A Novel Modulator of the Th1 Cytokine Response. Immunity 10:127-136

Oba M., Thangavelu G., Dahghan-Banadaky M., Ambrose DJ. (2009) Unprocessed Whole Flaxseed is as effective as Dry-Rolled Flaxseed at increasing $\alpha$-Linolenic Acid Concentration in Milk of Dairy Cows. Livestock Science 122:73-76.0

O’Banion MK., Winn VD., Young DA. (1992) cDNA Cloning and Functional Activity of a GlucocorticoidRegulated Inflammatory Cyclooxygenase. Proceedings of the National Academy of Sciences of the United States of America 89(11):4888-4892

O'Connor TM., O'Halloran DJ., Shanahan F. (2000) The Stress Response and the Hypothalamic-Pituitary-Adrenal Axis: From Molecular to Melancholia. QJM-Monthly Journal of the Association of Physicians 6:323-333

O’Leary S., Jasper MJ., Robertson SA., Armstrong DT. (2006) Seminal Plasma Regulates Ovarian Progesterone Production, Leukocyte Recruitment and Follicular Cell Responses in the Pig. Reproduction 132:147-158

O'Neill C. (2008) The Potential Roles for Embryotrophic Ligands in Preimplantation Embryo Development. Human Reproduction Update 14(3):275-288

Obata T., Nagakura T., Masaki T., Meakawa K., Yamashita K. (1999) Eicosapentaenoic Acid Inhibits Prostaglandin D2 Generation by Inhibiting Cyclo-Oxyganse-2 in Cultured Human Mast Cells. Clinical and Experimental Allergy 29(8):1129-1135

Oderup C., Cederbom L., Makowsak A., Cilio CM., Ivars F. (2006) Cytotoxic T Lymphocyte Antigen-4-Dependent Down-Modulation of Costimulatory Molecules on Dendritic Cells in CD4+ CD25+ Regulatory T-Cell-Mediated Suppression. Immunology 118(2):240-249

Ohira T. Arita M., Omori K., Recchiuti A., Van Dyke TE., Serhan CN. (2010) Resolvin E1 Receptor Activation Signals Phosphorylation and Phagocytosis. Journal of Biological Chemistry 285(5):3451-3461

Onishi ., Fehervari Z., Yamaguchi T., Sakaguchi S. (2008) Foxp3+ Natural Regulatory T Cells Preferentially Form Aggregates on Dendritic Cells in vitro and Actively Inhibit their Maturation. Proceedings of the National Academy of Sciences of the United States of America 105(29):10113-10118

Oever Jt., Kox M., van de Veerdonk FL., Mothapo KM., Slavcovici A., Jansen TL., Tweehuysen L., GiamarellosBourboulis EJ., Schneeberger PM., Wever PC., Stoffels M., Simon A., van der Meer JWM., Johnson MD., Kullberg B-J., Pickkers P., Pachot A., Joosten LAB., Netea MG. (2014) The Discriminative Capacity of Soluble Toll-Like Receptor (sTLR)2 and sTLR4 in Inflammatory Diseases. BMC Immunology 15:55 
Pace KE., Lee C., Stewart PL., Baum LG. (1999) Restricted Receptor Segregation into Membrane Microdomains Occurs on Human T Cells during Apoptosis Induced by Galectin-1. Journal of Immunology 163(7):3801-3811

Palmquist DL. And Jenkins TC. (1980) Fat in Lactation Rations: Review. Journal of Dairy Science 63:1-14

Pampfer S., Moulaert B., Vanderheyden I., Wuu YD., Se Hertogh R. (1994a) Effect of Tumor Necrosis Factor Alpha on Rat Blastocyst Growth and Glucose Metabolism. Journal of Reproduction and Fertility 101:199-206

Pampfer S., Wuu YD., Vanderheyden I., Hertogh RD. (1994b) Expression of Tumor Necrosis Factor-alpha (TNF alpha) Receptors and Selective Effect of TNF Alpha on the Inner Cell Mass in Mouse Blastocyst. Endocrinology 134:206-212

Pampfer S., Vanderheyden I., Vesela J., De Hertogh R. (1995) Neutralization of Tumor Necrosis Factor Alpha (TNF alpha) Action on Cell Proliferation in Rat Blastocysts by Antisense Oligodeoxyribonecleotides Directed Against TNF Alpha p60 Receptor. Biology of Reproduction 52(6):1316-1326

Pampfer S., Vanderheyden I., McCracken JE., Vesela J., De Hertogh R. (1997) Increased Cell Death in Rat Blastocyst Exposed to Maternal Diabetes in Utero and to High Glucose or Tumor Necrosis Factor-Alpha in vitro. Development 124(23):4827-4836

Paria BC., Reese J., Das SK., Dey Sk. (2002) Deciphering the Cross-Talk of Implantation: Advances and Challenges. Science 296:2185-2188

Park BS., Song DH., Kim HM., Choi BS., Lee H., Lee JO. (2009) The Structural Basis of Lipopolysaccharide Recognition by the TLR4-MD-2 Complex. Nature 1191-1195

Pate JL. (1995) Involvement of Immune Cells in Regulation of Ovarian Function. Journal of Reproduction and Fertility. Supplement 49:365-377

Paust S., Lu L., McCarty N., Cantor H. (2004) Engagement of B7 on Effector T Cells by Regulatory T Cells Prevents Autoimmune Disease. Proceedings of the National Academy of Sciences of the United States of America 101(28):10398-10403

Peltier MR., Liu WJ., Hansen PJ. (2000) Regulation of Lymphocyte Proliferation by Uterine Serpin: Interleukin-2 mRNA Production, CD25 Expression and Responsiveness to Interleukin-2. Proceedings of the National Society of Experimental Biology and Medicine 223:75-81

Perillo NL., Pace KE., Seilhamer JJ., Baum LG. (1995) Apoptosis of T Cells Mediated by Galectin-1. Nature 378(6558):736-739

Perlstein RS., Mougey EH., Jackson WE., Neta R. (1991) Interleukin-1 and Interleukin- 6 Act Synergistically to Stimulate the Release of Adrenocorticotropic Hormone in vivo. Lymphokine Cytokine Research 10(1-2):141-146

Perlstein RS., Whitnall MH., Abrams JS.,Mougey EH., Neta R. (1993) Synergistic Roles of Interleukin-6, Interleukin-1, and Tumor Necrosis Factor in Adrenocorticotropin Response to Bacterial Lipopolysaccharide in vivo. Endocrinology 132(3):946-952

Perregaux D. and Gabel CA. (1994) Interleukin-1 beta Maturation and Release in Response to ATP and Nigericin. Evidence that Potassium Depletion Mediated by these Agents is a Necessary and Common Feature of their Activity. Journal of Biological Chemistry 269(21): 15195-15203

Pasare C. and Medzhitov R. (2003) Toll Pathway-Dependent Blockade of CD4+CD25+ T Cell-Mediated Suppression by Dendritic Cells. Science 299:1033-1036

Petersen HH., Nielsen JP., Heegaard PMH. (2004) Application of Acute Phase Protein Measurements in Veterinary Clinical Chemistry. Veterinary Research 35(2):163-187 
Petit HV. (2002) Digestion, Milk Production, Milk Composition, and Blood Composition of Dairy Cows Fed Whole Flaxseed. Journal of Dairy Science 85:1482-1490

Petit HV., and Côrtes C. (2010) Milk Production and Composition, Milk Fatty Acid Profile, and Blood Composition of Dairy Cows Fed Whole or Ground Flaxseed in the First Half of Lactation. Animal Feed Science and Technology 158:36-43

Petit HV., Germiquet C., Lebel D. (2004) Effect of Feeding Whole, Unprocessed Sunflower Seeds and Flaxseed on Milk Production, Milk Composition, and prostaglandin Secretion in Dairy Cows. Journal of Dairy Science 87:38893898

Petit, H.V., and Twagiramungu, H. (2006) Conception Rate and Reproductive Function of Dairy Cows Fed Different Fat Sources. Theriogenology 66:1316-1324

Pétrilli V., Papin S., Dostert C., Mayor A., Martinon F., Tschopp J. (2007) Activation of the NALP3 Inflammasome is Triggered by Low Intracellular Potassium Concentration. Cell Death and Differentiation 14(9):1583-1589

Petroff MG., Petroff BK., Pate JL. (2001) Mechanisms of Cytokine-Induced Death of Cultured Bovine Luteal Cells. Reproduction 121: 753-760

Phillips B., Knisley K., Weitlauf KD., Dorsett J., Lee V., Weitlauf H. (1996) Differential Expression of two betaGalactoside-Binding Lectins in the Reproductive Tracts of Pregnant Mice. Biology of Reproduction 55(3):548-558

Piccinni MP., Giudizi MG., Biagiotii R., Beloni L., Giannarini L., Sampognaro S., Parronchi P., Manetti R., Annunziato F., Livi C. (1995) Progesterone Favors the Development of Human T Helper Cells Producing Th2-type Cytokines and Promotes both IL-4 Production and Membrane CD30 Expression in Established Th1 Cells Clones.

Journal of Immunology 155:128-133

Piccinni MP., Beloni L., Livi C., Maggi E., Scarselli GF., Romagnani S. (1998) Defective Production of both Leukemia Inhibitory Factor and Type 2 T-Helper Cytokines by Decidual T Cells in Unexplained Recurrent Abortions. Nature Medicine 4:1020-1024

Piccinni MP., Scaletti C., Vultaggio A., Maggi E., Romagnani S. (2001) Defective Production of LIF, M-CSF and Th2-type Cytokines by T cells at Fetomaternal Interface is Associated with Pregnancy Loss. Journal of Reproductive Immunology 52:35-43

Pinedo PJ., Galvão KN., Seabury CM. (2013) Innate immune Gene Variation and Differential Susceptibility to Uterine Diseases in Holstein Cows. Theriogenology 80: 384-390

Platanias LC. (2005) Mechanisms of Type I and Type II Interferon Mediated Signaling. Natuer Reviews immunology 5:375-386

Polanczyk MJ., Carson BD., Subramanian S., Afentoulis M., Vandenbark AA., Ziegler SF., Offner H. (2004) Cutting Edge: Estrogen Drives Expansion of the CD4+CD25+ Regulatory T Cell Compartment. Journal of Immunology 173(4):2227-2230

Pot C., Jin H., Awasthi A., Liu SM., Lai CY., Madan R., Sharpe AH., Karp CL., Miaw SC., Ho IC., Kuchroo VK. (2009) Cutting Edge: IL-27 Induces the Transcription Factor c-Maf, Cytokine IL-21, and the Costimulatory Receptor ICOS that Coordinately Act Together to Promote Differentiation of IL-10- Producing Tr1 Cells. Journal of Immunology 183(2):797-801

Pouliot M., Fiset ME., Masse M., Naccache PH., Borgeat P. (2002) Adenosine Up-Regulates Cyclooxygenase-2 in Human Granulocytes: Impact on the Balance of Eicosanoid Generation. Journal of Immunology 169:5279-5286

Prins JR., Zhang B., Schjenken JE., Guerin LR., Barry SC., Robertson SA. (2015) Unstable Foxp3+ Regulatory T Cells and Altered Dendritic Cells are Associated with Lipopolysaccharide-Induced Fetal Loss in Pregnant Interleukin 10-Deficient Mice. Biology of Reproduction 93(4):95 
Purasiri P., Mckechnie A., Heys SD., Eremin O. (1997) Modulation in vitro of Human Natural Cytotoxicity, Lymphocyte Proliferative Response to Mitogens and Cytokine Production be Essential Fatty Acids. Immunology 92(2):166-172

Putney DJ., Drost M., Thatcher WW. (1988) Embryonic Development in Superovualted Dairy Cattle Exposed to Elevated Ambient Temperatures between Days 1 to 7 Post Insemination. Theriogenology 32(2):195-209

Rabinovich GA. (1999) Galectins: An Evolutionarily Conserved Family of Animal Lectins with Multifunctional Properties; A Trip from the Gene to Clinical Therapy. Cell Death and Differentiation 6:711-721

Rabinovich GA. (2000) Evidence of a Role for Galectin-1 in Acute Inflammation. European Journal of Immunology 30(5):1331-1339

Rabinovich GA. and Ilarregui JM. (2009) Conveying Glycan Information into T-cell Homeostatic Programs: A Challenging Role for Galectin-1 in Inflammatory and Tumor Microenvironments. Immunological Reviews 230:144159

Rabinovich GA., and Toscano MA. (2009) Turning 'Sweet' on Immunity: Galectin-Glycan Interactions in Immune Tolerance and Inflammation. Nature Reviews Immunology 9:338-352

Rabinovich GA., Iglesias MM., Modesti NM., Castagna LF., Wolfenstein-Todel C., Riera CM., Sotomayor CE. (1998) Activated Rat Macrophages Produce a Galectin-1 Like Protein that Induces Apoptosis of T Cells: Biochemical and Functional Characterization. Journal of Immunology 160(10):4831-4840

Rabinovich GA., Ariel A., Hershkoviz R., Hirabayashi J., Kasai K-I., Lider O. (1999) Specific Inhibition of T-cell Adhesion to Extracellular Matrix and Proinflammatory Cytokine Secretion by Human Recombinant Galectin-1. Immunology 97:100-106

Rabinovich GA., Alonso CR., Sotomayor CE., Durand S., Bocco JL., Riera CM. (2000) Molecular Mechanisms Implicated in Galectin-1-Induced Apoptosis: Activation of the AP-1 Transcription Factor and Downregulation of Bcl-2. Cell Death and Differentiation 7(8):747-753

Rabinovich GA., Baum LG., Tinari N., Paganelli R., Natoli C., Liu F-T., Lacobelli S. (2002) Galectins and their Ligands: Amplifiers, Silencers or Tuners of the Inflammatory Response? Trends in Immunology 23(6):313-320

Rabinovich GA., Liu FT., Hirashima M., Anderson A. (2007) An Emerging Role for Galectins in Tuning the Immune Response: Lessons from Experimental Models of Inflammatory Disease, Autoimmunity and Cancer. Scandinavian Journal of immunology 66:143-158

Radulovic M., Dautzenberg FM., Sydow S., Radulovic J., Spiess J. (1999) Corticotropin-Releasing Factor Receptor 1 in Mouse Spleen: Expression after Immune Stimulation and Identification of Receptor-Bearing Cells. Journal of Immunology 162(5):3013-3021

Raetz CR. (1993) Bacterial Endotoxins: Extra Ordinary Lipids that Activate Eukaryotic Signal Transduction. Journal of Bacteriology 175(18)5745-5753

Ramakers C., Ruijter JM., Lekanne Deprez RH., Moorman AFM. (2003) Assumption-Free Analysis of Quantitative Real-Time Polymerase Chain Reaction (PCR) Data. Neuroscience Letters 339:62-66

Ramhorst RE., García VE., Corigliano A., Rabinovich GA., Fainboim L. (2004) Identification of RANTES as a Novel Immunomodulator of the Maternal Allogeneic Response. Clinical Immunology 110:71-80

Ramhorst R., Fraccaroli L., Aldo P., Alvero AB., Cardenas I., Leirós CP., Mor G. (2012a) Modulation and Recruitment of Inducible Regulatory T Cells by First Trimester Trophoblast Cells. American Journal of Reproductive Immunology 67:17-27 
Ramhorst R., Giribaldi L., Fraccaroli L., Toscano MA., Stupirski JC., Romero MD., Durand ES., Rubinstein N., Blaschitz A., Sedlmayr P., Genti-Raimondi S., Fainboim L., Rabinovich GA. (2012b) Galectin-1 Confers Immune Privilege to Human Trophoblast: Implications in Recurrent Fetal Loss. Glycobiology 22(10):1374-1386

Ramíez F., Fowell DJ., Puklavec M., Simmonds S., Mason D. (1996) Glucocorticoids Promote a TH2 Cytokine Response by CD4+ T Cells in Vitro. Journal of Immunology 156(7):2406-2412

Randall GW., O'Connor EF., Gantt PA. (1991) Synergy between Tumor Necrosis Factor and Endotoxin decreases Early Embryo Development in vitro. Journal of in vitro Fertilization and Embryo Transfer: IVF 8(6):304-307

Rauk PN., Friebe-Hoffmann U., Winebrenner LD., Chiao JP. (2001) Interleukin-6 Up-Regulates the Oxytocin Receptor in Cultrured Uterine Smooth Muscle Cells. American Journal of Reproductive Immunology 45(3):148-153

Recchiuti A., Krishnamoorthy S., Fredman G., Chiang N., Serhan CN. (2011) MicroRNAs in Resolution of Acute Inflammation: Identification of Novel Resolvin D1-miRNA Circuits. FASEB Journal 25(2):544-560

Rees D., Miles EA., Banerjee T., Wells SJ., Roynette CE., Wahle KWJW., Calder PC. (2006) Dose-Related Effects of Eicosapentaenoic Acid on Innate Immune Function in Healthy Humans: A Comparison of Young and older Men. American Journal of Clinical Nutrition 83:331-342

Reiner SL. (2007) Development in Motion: Helper T Cells at Work. Cell 129:33-36

Remes LF., Rodriguez RC., Sabatté J., Cabrini M., Jancic O., Ostrowski M., Merlotti A., Gonzalez H., Alonso A., Pasqualini RA., Davio C., Geffner J., Ceballos A. (2012) Semen Promotes the Differentiation of Tolerogenic Dendritic Cells. Journal of Immunology 189(10):4777-4786

Renaud SJ. and Graham CH. (2008) The Role of Macrophages in Utero-Placental Interactions during Normal and Pathological Pregnancy. Immunological Investigations 37(5):535-564

Renz H., Gong JH., Schmidt A., Nain M., Gemsa D. (1988) Release of Tumor Necrosis Factor-Alpha from Macrophages. Enhancement and Suppression are Dose-Dependently Regulated by Prostaglandin E2 and Cyclic Nucleotides. Journal of Immunology 141(7):2388-2393

Ribeiro ES., Greco LF., Bisinotto RS., Lima FS., Thatcher WW., Santos JEP. (2016a) Biology of Preimplantation Conceptus at the Onset of Elongation in Dairy Cows. Biology of Reproduction 94(4):97:1-18

Ribeiro ES., Gomes G., Greco LF., Cerri RLA., Vieira-Neto A,. Monteiro PLJ Jr., Lima FS., Bisinotto RS., Thatcher WW., Santos JEP. (2016b) Carryover Effect of Postpartum Inflammatory Diseases on Developmental Biology and Fertility in Lactating Dairy Cows. Journal of Dairy Science 99:2201-2220

Ribeiro ES., Santos JEP., Thatcher WW. (2016c) Role of Lipids on Elongation of the Preimplantation Conceptus in Ruminants. Reproduction 152:R115-R126

Ricote M., Li AC., Willson TM., Kelly CJ., Glass CK. (1998) The Peroxisome Proliferator-Activated ReceptorGamma is a Negative Regulator of Macrophage Activation. Nature 319(6662):79-82

Rietschel ET., Kirikae T., Schade FU., Mamat U., Schmidit G., Loppnow H., Ulmer AJ., Zähringer U., Seydel U., Di Padova F., Scherier M., Brade H. (1994) Bacterial Endotoxins: Molecular Relationships of Structure to Activity and Function. FASEB Journal 8(2):217-225

Rincón M., Tugores A., López-Rivas A., Silva A., Alonso M., De Landázuri MO., López-Botet M. (1988) Prostaglandin E2 and the Increase of Intracellular cAMP Inhibit the Expression of Interleukin 2 Receptors in Human $\mathrm{T}$ cells. European Journal of Immunology 18(110:1791-1796

Riollet C., Rainard P., Poutrel B. (2001) Cell Subpopulations and Cytokine Expression in Cow Milk in Response to Chronic Staphylococcus aureus Infection. Journal of Dairy Science 84:1077-1084 
Rizos D., Carter F., Besenfelder U., Havlicek V., Lonergan P. (2010) Contribution of the Female Reproductive Tract to Low Fertility in Postpartum Lactating Dairy Cows. Journal of Dairy Science 93:1022-1029

Roberts RM and Bazer FW. (1988) The Functions of Uterine Secretions. Journal of Reproduction and Fertility 82(2):875-892

Roberts RM., Liu L., Guo Q., Leaman D., Bixby J. (1998) The Evolution of the Type I Interferons. Journal of Interferon and Cytokine Research 18:805-816

Roberts RM., Ezashi T., Rosenfeld CS., Ealy AD., Kubisch HM. (2003) Evolution of the Interferon Tau Genes and their Promoters, and Maternal-Trophoblast Interactions in Control of their Expression. Reproduction Supplement 61:239-251

Roberts RM., Chen Y., Ezashi T., Walker AM. (2008) Interferons and the Maternal-Conceptus Dialog in Mammals. Seminars in Cell \& Developmental Biology 19:170-177

Robertson SA. (2000) Control of the Immunological Environment of the Uterus. Reviews of Reproduction 5(3):164174

Robertson SA. (2007) GM-CSF Regulation of Embryo Development and Pregnancy. Cytokine and Growth factor Reviews 18(3-4):287-298

Robertson SA., Mayrhofer G., Seamark RF. (1992a) Uterine Epithelial Cells Synthesize Granulocyte-Macrophage Colony-Stimulating Factor and Interleukin-6 in Pregnant and Non-Pregnant Mice. Biology of Reproduction 46(6):1069-1079

Robertson SA., Brännström M., Seamark RF. (1992b) Cytokine in Rodent Reproduction and the CytokineEndocrine Interaction. Current Opinion in Immunology 4:585-590

Robertson SA., Mau VJ., Tremellen KP., Seamark RF. (1996) Role of High Molecular Weight Seminal Vesicle Proteins in Eliciting the Uterine Inflammatory Response to Semen in Mice. Journal of Reproduction and Fertility 107(2):265-277

Robertson SA., Allanson M., Mau VJ. (1998) Molecular Regulation of Uterine Leukocyte Recruitment during Early Pregnancy in the Mouse. Trophoblast Research 19:101-119

Robertson SA. and Sharkey DJ. (2001) The Role of Semen in Induction of Maternal Immune Tolerance to Pregnancy. Seminars in Immunology 13(4):243-254

Robertson SA., Sjöblom C., Jasper MJ., Norman RJ., Seamark RF. (2001) Granulocyte-Macrophage ColonyStimulating Factor Promotes Glucose Transport and Blastomere Viability in Murine Preimplantation Embryos. Biology of Reproduction 64(4):1206-1215

Robertson SA., Ingman WV., O’Leary S., Sharkey DJ., Tremellen KP. (2002) Transforming Growth Factor $\beta$-A Mediator of Immune Deviation in Seminal Plasma. Journal of Reproductive Immunology 57:109-128

Robertson SA., Care AS., Skinner RJ. (2007) Interleukin 10 Regulates Inflammatory Cytokine Synthesis to Protect Against Lipopolysaccharide-Induced Abortion and Fetal Growth Restriction in Mice. Biology of Reproduction 76(5):738-748

Robertson SA., Guerin LR., Bromfield JJ., Branson KM., Ahlström AC., Care AS. (2009) Seminal Fluid Drives Expansion of the CD4+CD25+ T Regulatory Cell Pool and Induces Tolerance to Paternal Alloantigens in Mice. Biology of Reproduction 80:1036-1045

Robertson SA., Christiaens I., Dorían CL., Zaragoza DB., Care AS., Banks AM., Olson DM. (2010) Interleukin-6 is an Essential Determinant of on-time Parturition in the Mouse. Endocrinology 151(8):3996-4006 
Robertson SA., Chin PY., Glynn DJ., Thompson JG. (2011) Peri-Conceptual Cytokines-Setting the Trajectory for Embryo Implantation, Pregnancy and Beyond. American Journal of Reproductive Immunology 66(Suppl 1):2-10

Robertson SA., Prins JR., Sharkey DJ., Moldenhauer LM. (2013) Seminal Fluid and the Generation of Regulatory T Cells for Embryo Implantation. American Journal of Reproductive immunology 69:315-330

Robertson SA. and Moldenhauer LM. (2014) Immunological Determinants of Implantation Success. International Journal of Developmental Biology 58:205-217

Robinson D., Shibuya K., Mui A., Zonin F., Murphy E., Sana T., Hartley SB., Menon S., Kastelein R., Bazan F., O'Garra A. (1997) IGIF does not Drive Th1 Development by Synergizes with IL-12 for Interferon- $\gamma$ Production and Activates IRAK and NF-кB. Immunity 7(4):571-581

Roche JF. (2006) The Effect of Nutritional Management of the Dairy Cow on Reproductive Efficiency. Animal Reproductive Science 96:282-296

Rodriquez-Martinez H., McKenna D., Weston PG., Whitmore HL., Gustafsson BK. (1987) Uterine Motility in the Cow during the Estrous Cycle. I. Spontaneous Activity. Theriogenology 27(2):337-348

Rosario GX. Stewart CL. (2016) The Multifaceted Actions of Leukaemia Inhibitory Factor in Mediating Uterine Receptivity and Embryo Implantation. American Journal of Reproductive Immunology 75:246-255

Rouas-Freiss N., Goncalves R M-B., Menier C., Dausset J., Carosella ED. (1997) Direct Evidence to Support the Role of HLA-G in Protecting the Fetus from Maternal Uterine Natural Killer Cytolysis. Proccedings of the National Academy of Sciences of the United States of America 94(21):115200-11525

Rowe JH., Ertelt JM., Aguilera MN., Farrar MA., Way SS (2011) Foxp3 ${ }^{+}$Regulatory T Cell Expansion Required for Sustaining Pregnancy Compromises Host Defense against Prenatal Bacterial Pathogens. Cell Host and Microbe 10:54-64

Rowe JH., Ertelt JM., Xin L., Way SS. (2012a) Pregnancy Imprints Regulatory Memory that sustains Anergy to Fetal Antigen. Nature 490:102-106

Rowe JH., Ertelt JM., Xin L., Way SS. (2012b) Listeria monocytogenes Cytoplasmic Entry Induces Fetal Wastage by Disrupting Maternal Foxp3+ Regulatory T Cell-Sustained Fetal tolerance. PLoS Pathogens 8(8): e1002873

Rozen S. and Skaletsky H. (2000) Primer3 on the WWW for General Users and for Biological Programmers. Methods in Molecular Biology 132:365-386

Sadeghi S., Wallace FA., Calder PC. (1999) Dietary Lipids Modify the Cytokine Response to Bacterial Lipopolysaccharide in Mice. Immunology 96(3):404-410

Sadik CN., Kim ND., Luster AD. (2011) Neutrophils Cascading their Way to Inflammation. Trends in Immunology 32(10):452-460

Saeed SA., Mahmood F., Shah BH., Gilani AH. (1997) The Inhibition of Prostaglandin Biosynthesis by Human Haptoglobin and its Relationship with Haemoglobin Binding. Biochemical Society Transactions 25(4):S618

Saito S. (2000) Cytokine Network at the Feto-Maternal Interface. Journal of Reproductive Immunology 47:87-103

Saito S., Matsuura M., Tominaga K., Kirikae T., Nakano M. (2000) Important Role of Membrane Associated CD14 in the Induction of INF-beta and Subsequent Nitric Oxide Production by Murine Macrophages in Response to Bacterial Lipopolysaccharide. European Journal of Biochemistry 267:37-45

Saito S., Nakashima A., Shima T., Ito M. (2010) Th1/Th2/Th17 and Regulatory T-Cell Paradigm in Pregnancy. American Journal of Reproductive Immunology 63:601-610 
Sato K., Yamashita N., Baba M., Matsuyama T. (2003) Modified Myeloid Dendritic Cells Act as Regulatory Dendritic Cells to Induce Anergic and Regulatory T Cells. Blood 101(9):3581-3589

Saitoh T., Fujita N., Jang MH., Uematsu S., Yang BG., Satoh T., Omori H., Noda T., Yamamoto N., Komatsu M., Tanaka K., Kawai T., Taujimura T., Takeuchi O., Yoshimori T., Akira S. (2008) Loss of the Autophagy Protein Atg16L1 Enhances Endotoxin-Induced IL-1beta Production. Nature 456(7219):264-268

Sakaguchi S. (2004) Naturally Arising CD4+ Regulatory T Cells for Immunologic Self-Tolerance and Negative Control of Immune Responses. Annual Reviews in Immunology 22:534-562

Salamonsen LA., Dimitriadis E., Robb L., (2000) Cytokines in Implantation. Seminars in Reproductive Medicine 18(3):299-310

Samstein RM., Josefowicz SZ., Arvey A., Treuting PM., Rudensky AY. (2012) Extrathymic Generation of Regulatory T Cells in Placental Mammals Mitigates Maternal-Fetal Conflict. Cell 150:29-38

Samy ET., Setiady YY., Ohno K., Pramoonjago P., Sharp C., Tung KS. (2006) The Role of Physiological SelfAntigen in the Acquisition and Maintenance of Regulatory T-cell Function. Immunology Reviews 212:170-184

Sandra O., Constant F., Carvalho AV., Eozénou C., Valour D., Mauffré V., Hue I., Charpigny G. (2015) Maternal Organism and Embryo Biosensoring: Insights from Ruminants. Journal of Reproductive Immunology 108:105-113

Santner-Nanan B., Straubinger K., Hsu P., Parnell G., Tang B., Xu B., Makris A., Hennessy A., Peek MJ., Busch DH., da Costa CP., Nanan R. (2013) Fetal-Maternal Alignment of Regulatory T Cells Correlates with IL-10 and Bcl-2 Upregulation in Pregnancy. The Journal of Immunology 191:145-153

Santos JSP. And Ribeiro ES. (2014) Impact of Animal Health on Reproduction of Dairy Cows. Animal Reproduction 11(3):254-269

Santos JEP., Cerri RLA., Ballou MA., Higginbotham GE., Kirk JH. (2004b) Effect of Timing of First Clinical Mastitis Occurrence on Lactational and Reproductive Performance of Holstein Dairy Cows. Animal Reproduction Science 80:31-45

Santos JEP., Bilby TR., Thatcher WW., Staples CR., Silvestre FT. (2008) Long Chain Fatty Acids of Diet as Factors Influencing Reproduction in Cattle. Reproduction in Domestic Animals 43:23-30

Sapolsky R., Rivier C., Yamamoto G., Plotsky P., Vale W. (1987) Interleukin-1 Stimulates the Secretion of Hypothalamic Corticotropin-Releasing Factor. Science 238(4826):522-524

Sargent JR. (1997) Fish Oils and Human Diet. British Journal of Nutrition 78 (suppl. 1):S5-S13

Sartori R., Bastos MR., Wiltbank MC. (2010) Factors affecting Fertilization and Early Embryo Quality in Singleand Superovulated Dairy Cattle. Reproduction, Fertility, and Development 22:151-158

Sasaki Y., Sakai M., Miyazaki S., Higuma S., Shiozaki A., Saito S. (2004) Decidual and Peripheral Blood CD4+CD25+ Regulatory T Cells in Early Pregnancy Subjects and Spontaneous Abortion Cases. Molecular Human Reproduction 10(5)347-353

Satterfield MC., Song G., Kochan KJ., Riggs PK., Simmons RM., Elsik CG., Adelson DL., Bazer FW., ZhouH., Spencer TE. (2009) Discovery of Candidate Genes and Pathways in the Endometrium Regulating Ovine Blastocyst Growth and Conceptus Elongation. Physiological Genomics 39(2):85-99

Savill J. and Fadok V. (2000) Corpse Clearance Defines the Meaning of Cell Death. Nature 407(6805):784-788

Scandella E., Men Y., Legler DF., Gillessen S., Prikler L., Ludewig B., Groettrup M. (2004) CCL19/CCL21Triggered Signal Transduction and Migration of Dendritic Cells Requires Prostaglandin E2. Blood 103(5):15951601 
Scapini P., Lapinet-Vera JA., Gasperini S., Calzetti F., Bazzoni F., Cassatella MA. (2000) The Neutrophil as a Cellular Source of Chemokines. Immunological Reviews 177:195-203

Scenna FN., Edwards JL., Rohrbach NR., Hockett ME., Saxton AM., Schrick FN. (2004) Deteimental effects of Prostaglandin F2alpha on Preimplantation Bovine Embryos. Prostaglandins \& Other Lipid Mediators 73(3-4):215226

Schaiff WT., Knapp Jr FF., Barak Y., Biron-Shental, T., Nelson DM., Sadovsky Y. (2007) Ligand-Activated Peroxisome Proliferator Activated Receptor $\gamma$ Alters Placental Morphology and Placental Fatty Acid Uptake in Mice. Endocrinology 148(8):3625-3634

Scheffold A., Hühn J., Höfer T. (2005) Regulation of CD4+CD25+ Regulatory T Cell Activity: it takes (IL-) Two to Tango. European Journal of Immunology 35(5):1336-1341

Scher JU. and Pillinger MH. (2005) 15d-PGJ2: the Anti-Inflammatory Prostaglandin? Clinical Immunology 114(2):100-109

Scott JL., Ketheesan N., Summers PM. (2006) Leukocyte Population Changes in the Reproductive Tract of the Ewe in Response to Insemination. Reproduction, Fertility, and Development 18(6):627-634

Sentman CL., Meadows SK., Wira CR., Eriksson M. (2004) Recruitment of Uterine NK Cells: Induction of CXC Chemokine Ligands 10 and 11 in Human Endometrium by Estradiol and Progesterone. Journal of Immunology 173(11):6760-6766

Shen XH., Han YJ., Zhang DX., Cui XS., Kim NH. (2009) A Link between the Interleukin-6/Stat3 Anti-Apoptotic Pathway and MicroRNA-21 in Preimplantation Mouse Embryos. Molecular Reproduction and Development 76(9):854-862

Scholljegerdes E. and Kronberg S. (2008) Influence of Level of Supplemental Whole Flaxseed on Forage Intake and Site and Extent of Digestion in Beef Heifers Consuming Native Grass Hay. Journal of Animal Science 86:23102320

Schroder K. and Tschopp J. (2010) The Inflammasomes. Cell 140(6):821-832

Schroder JW., Bauer ML., Bork NR. (2014) Effect of Flaxseed Physical Form on Digestibility of Lactation Diets Fed to Holstein Steers. Journal of Dairy Science 97(9):5718-5728

Schwab JM., Chiang N., Arita M., Serhan CN. (2007) Resolvin E1 and Protectin D1 Activate InflammationResolution Programmes. Nature 447(7146):869-874

Seals RC., Lemaster JW., Hopkins FM., Schrick FN. (1998) Effects of Elevated Concentrations of Prostaglandin F2 Alpha on Pregnancy Rates in Progestogen Supplemented Cattle. Prostaglandins and Other Lipid Mediators 56(56):377-389

Segal AW. (2005) How Neutrophils Kill Microbes. Annual Review of Immunology 23:197-223

Sentman CL., Meadows SK., Wira CR., Eriksson M. (2004) Recruitment of Uterine NK Cells: Induction of CXC Chemokine Ligands 10 and 11 in Human Endometrium by Estradiol and Progesterone. Journal of Immunology 173(11):6760-6766

Serhan CN. (2007) Resolution Phase of Inflammation: Novel Endogenous Anti-Inflammatory and Proresolving Lipid Mediators and Pathways. Annual Reviews of Immunology 25:101-137

Serhan CN. (2014) Pro-Resolving Lipid Mediators are Leads for Resolution Physiology. Nature 510(7503):90-101

Serhan CN. and Chiang N. (2013) Resolution Phase Lipid Mediators of Inflammation: Agonists of Resolution. Current Opinion in Pharmacology 13(4):632-640 
Serhan CN. and Petasis NA. (2011) Resolvins and Protectins in Inflammation-Resolution. Chemical Reviews 111:5922-5943

Serhan CN. and Savill J. (2005) Resolution of Inflammation: The Beginning Programs the End. Nature Immunology 6(12):1191-1197

Serhan CN., Maddox JF., Petasis NA., Akritopoulou-Zanze I., Papayianni A., Brady HR., Colgan SP., Madara JL. (1995) Design of Lipoxin A4 Stable Analogs that Block Transmigration and Adhesion of Human Neutrophils. Biochemistry 34(44):14609-14615

Serhan CN., Chiang N., Dalli J., Levy BD. (2014) Lipid Mediators in the Resolution of Inflammation. Cold Spring Harbor Perspectives in Biology 7(2):a016311

Shalom-Barak T., Zhang X., Chu T., Schaiff WT., Reddy JK., Xu J., Sadovsky Y., Barak Y. (2012) Placental PPAR $\gamma$ Regulated Spatiotemporally Diverse Genes and a Unique Metabolic Network. Developmental Biology 372:143-155

Sharkey AM., Dellow K., Blayney M., Macnamee M., Charnock-Jones S., Smith SK. (1995) Stage-Specific Expression of Cytokine and Receptor Messenger Ribonucleic Acids in Human Preimplantation Embryos. Biology of Reproduction 53(4):974-981

Sharkey DJ., Macpherson AM., Tremellen KP., Robertson SA. (2007) Seminal Plasma Differentially Regulates Inflammatory Cytokine Gene Expression in Human Cervical and Vaginal Epithelial Cells. Molecular Human Reproduction 13(7):491-501

Sharma S. (2014) Natural Killer Cells and Regulatory T Cells in Early Pregnancy Loss. The International Journal of Developmental Biology 58:219-229

Sharma MD., Baban B., Chandler P., Hou D-Y., Singh N., Yagita H., Azuma M., Blazar BR., Mellor AL., Munn DH. (2007) Plasmacytoid Dendritic Cells from Mouse Tumor-Draining Lymph Nodes Directly Activate Mature Tregs via Indoleamine 2,3-Dioxygenase. Journal of Clinical Investigation 117(9):2570-2582

Sharpe AH., Wherry EJ., Ahmed R., Freeman GJ. (2007) The Function of Programmed Cell Death 1 and its Ligands in Regulating Autoimmunity and Infection. Nature Immunology 8(3):239-245

Shaw JL., Day SK., Critchley HO., Horne AW. (2010) Current Knowledge of the Aetiology of Human Tubal Ectopic Pregnancy. Human Reproduction Update 16(4):432-444

Shaw JL., Fitch P., Cartwright J., Entrican G., Schwarze J., Critchley HO., Horne AW. (2011) Lymphoid and Myeloid Cell Populations in the Non-Pregnant Human Fallopian Tube and in Ectopic Pregnancy. Journal of Reproductive Immunology 89:84-91

Sheldon IM. and Dobson H. (2004) Postpartum Uterine Health in Cattle. Animal Reproduction Science 82-83:295306

Sheldon IM., Noakes DE., Rycroft A., Dobson H. (2001) Acute Phase Protein Responses to Uterine Bacterial Contamination in Cattle after Calving. The Veterinary Record 148 (6):172-175

Sheldon IM., Lewis GS., Leblanc S., Gilbert RO. (2006) Defining Postpartum Uterine Disease in Cattle. Theriogenology 65:1516-1530

Sheldon IM., Cronin J., Goetze L., Donofrio G., Schuberth H-S. (2009a) Defining Postpartum Uterine Disease and the Mechanisms of Infection and Immunity in the Female Reproductive Tract in Cattle. Biology of Reproduction $81: 1025-1032$ 
Sheldon IM., Price SB., Cronin J., Gilbert RO., Gadsby JE. (2009b) Mechanisms of Infertility Associated with Clinical and Subclinical Endometritis in High Producing Dairy Cattle. Reproduction in Domestic Animals 44 (Suppl. 3): $1-9$

Sheldon IM., Cronin JG., Healey GD., Gabler C., Heuwieser W., Streyl D., Bromfield JJ., Miyamoto A., Fergani C., Dobson H. (2014) Innate Immunity and Inflammation of the Bovine Female Reproductive Tract in Health and Disease. Reproduction 148(3):R41-51

Shepherd VL., Konish MG., Stahl P. (1985) Dexamethasone Increases Expresssion of Mannose Receptors and Decreases Extracellular Lysosomal Enzyme Accumulation in Macrophages. Journal of Biological Chemistry 260:160-164

Shepherd VL., Abdolrasulnia R., Garrett M., Cowan HB. (1990) Down-Regulation of Mannose Receptor Activity in Macrophages after Treatment with Lipopolysaccharide and Phorbol Esters. Journal of Immunology 145:1530-1536

Shevach EM. (2002) CD4+ CD25+ Suppressor T cells: More Questions than Answers. Nature Reviews Immunology

Shevach EM. (2009) Mechanisms of FOXP3+ T Regulatory Cell-Mediated Suppression. Immunity 30(5):636-645

Shi Y., Evans JE., Rock KL. (2003) Molecular Identification of a Danger Signal that Alerts the Immune System to Dying Cells. Nature 425(6957); 516-521

Shima T., Sasaki Y., Itoh M., Nakashima A., Ishii N., Sugamura K., Saito S. (2010) Regulatory T Cells are Necessary for Implantation and Maintenance of Early Pregnancy but not Late Pregnancy in Allogenic Mice. Journal of Reproductive Immunology 85(2):121-129

Shima T., Inada., Nakashima A., Saito S. (2012) Paternal Antigen-Specific Inducible Regulatory T cells are Increased just before Implantation by Seminal Fluid Priming. American Journal of Reproductive Immunology 96,12

Shinomiya S., Naraba H., Ueno A., Utsunomiya I., Maruyama T., Ohuchida S., Ushikubi F., Yuki K., Narumiya S., Sugimoto Y., Ichikawa A., Oh-ishi S. (2001) Regulation of TNF $\alpha$ and Interleukin-10 Production by Prostaglandins $\mathrm{I}_{2}$ and $\mathrm{E}_{2}$ : Studies with Prostaglandin Receptor-Deficient Mice and Prostaglandin E-Receptor Subtype-Selective Synthetic Agonists. Biochemical Pharmacology 61:1153-1160

Shiono Y., Mun HS., He N., Nakazaki Y., Fang H., Furuya M., Aosai F., Yano A. (2007) Maternal-Fetal Transmission of Toxoplasma gondii in Interferon-gamma Deficient Pregnant Mice. Parasitology International 56(2):141-148

Silver RM., Lohner WS., Daynes RA., Mitchell MD., Branch DW. (1994) lipopolysaccharide-Induced Fetal Death: The Role of Tumor-Necrosis Factor Alpha. Biology of Reproduction 50(5):1108-1112

Silvestre FT., Carvalho TSM., Crawford C., Santos JEP., Staples CR., Thatcher WW. (2008) Effects of Differential Supplementation of Calcium Salts of Fatty Acids (CSFAs) in lactating Dairy Cows on Plasma Acute Phase Proteins and Leukocyte Responses: Phagocytic and Oxidative Burst, CD62L/CD18 Expression and Cytokine Production. Biology of Reproduction 78:147 (abstract)

Silvestre, F.T., Carvalho, T.S.M., Francisco, N., Santos, J.E.P., Staples, C.R., Jenkins, T.C., Thatcher, W.W. (2011a) Effects of Differential Supplementation of Fatty Acids During the Peripartum and Breeding Periods of Holstein Cows: I. Uterine and Metabolic Responses, Reproduction, and Lactation. Journal of Dairy Science 94:189-204

Silvestre, F.T., Carvalho, T.S.M., Crawford, P.C., Santos, J.E.P., Staples, C.R., Jenkins, T.C., Thatcher, W.W. (2011b) Effects of Differential Supplementation of Fatty Acids During the Peripartum and Breeding Periods of Holstein Cows: II. Neutrophil Fatty Acids and Function, and Acute Phase Proteins. Journal of Dairy Science 94:2285-2301 
Simmons RM., Erikson DW., Kim J., Burghardt RC., Bazer FW., Johnson GA., Spencer TE. (2009) Insulin-Like Growth Factor Binding Protein-1 in the Ruminant Uterus: Potential Endometrial Marker and Regulator of Conceptus Elongation. Endocrinology 150:4295-4305

Simmons RM., Satterfield MC., Welsh Jr TH., Bazer FW., Spencer TE. (2010) HSD11B1, HSD11B2, PTGS2, and NR3C1 Expression in the Peri-Implanation Ovine Uterus: Effects of Pregnancy, Progesterone, and Interferon Tau. Biology of Reproduction 82:35-43

Simón C., Moreno C., Remohí J., pellicer A. (1998) Cytokines and Embryo Implantation. Journal of Reproductive Immunology 39(1-2):117-131

Simpson H., Robson SC., Bulmer Jn., Barber A., Lyall F. (2002) Transforming Growth Factor beta Expression in Human Placenta and Placental bed during Early Pregnancy. Placenta 23:44-58

Singh VK. (1989) Stimulatory Effect of Corticotropin-Releasing Neurohormone on Human Lymphocyte Proliferation and Interleukin-2 Receptor Expression. Journal of Neuroimmunology 23(3):257-262

Singh VK and Leu SJ. (1990) Enhancing effect of Corticotropin-Releasing Neurohormone on the Production of Interleukin-1 and Interleukin-2. Neuroscience Letters 120(2):151-154

Skarzynski DJ., Miyamoto Y., Okuda K. (2000) Production of Prostaglandin $F_{2 \alpha}$ by Cultured Bovine Endometrial Cells in Response to Tumor Necrosis Factor $\alpha$ : Cell Type Specificity and Intracellular Mechanisms. Biology of Reproduction 62(5): 1116-1120

Ślebodziński AB., Malinowski E., Lipczak W. (2002) Concentrations of Triidothyronine ( $\left.\mathrm{T}_{3}\right)$, Tumor Necrosis- $\alpha$ $(\mathrm{TNF} \alpha)$ and Interleukin-6 (IL-6) in Milk from Healthy and Naturally Infected Quarters of Cows. Research in Veterinary Science 72:17-21

Smith RJ. (1977) Modulation of Phagocytosis by and Lysosomal Enzyme Secretion from Guinea-Pig Neutrophils: Effect of Nonsteroid Anti-Inflammatory Agents and Prostaglandins. Journal of Pharmacology and Experimental Therapeutics 200(3):647-657

Smith WL., DeWitt DL., Garavito RM. (2000) Cyclooxygenases: Structural, Cellular, and Molecular Biology. Annual Reviews in Biochemistry 69:145-182

Snijdewint FG., Kaliński P., Wierenga EA., Bos JD., Kapsenberg ML. (1993) Prostaglandin E2 Differentially Modulates Cytokine Secretion Profiles of Human T Helper Lymphocytes. Journal of Immunology 150(12)53215329

Soder KJ., Brito AF., Rubano MD., Dell CJ. (2012) Effect of Incremental Flaxseed Supplementation of an Herbage Diet on Methane Output and Ruminal Fermentation in Continuous Culture. Journal of Dairy Science 95:3961-3969

Soehnlein O., Lindbom L., Weber C. (2009) Mechanisms Underlying Neutrophil-Mediated Monocyte Recruitment. Blood 114(21):4613-4623

Solier C., Aguerre-Girr M., Lenfant F., Campan A., Berrebi A., Rebmann V., Grosse-Wilde H., Le Bouteiller P. (2002) Secretion of Pro-Apoptotic Intron 4-Retaining Soluble HLA-G1 by Human Villous Trophoblast. European Journal of Immunology 32(12):3576-3586

Solle M., Labasi J., Perrehaux DG., Stam E., Petrushova N., Koller BH., Griffiths RJ., Gabel CA. (2001) Altered Cytokine Production in Mice Lacking P2X(7) Receptors. Journal of Biological Chemistry 276:125-132

Somerset DA., Zheng Y., Kilby MD., Sansom DM., Drayson MT. (2004) Normal Human Pregnancy is Associated with an Elevation in the Immune Suppressive CD25+CD4+ Regulatory T-Cell Subset. Immunology 112:38-43

Sommers KN. (2014) Immune Related Gene Expression of Parasite-Resistant Sheep during a Haemonchus contortus Infection. WVU Thesis UMI \# 1555016 
Song H., Lim H., Paria BC., Matsumoto H., Swift LL., Morrow J., Bonventre JV., Dey SK. (2002) Cytosolic Phospholipase $\mathrm{A}_{2 \alpha}$ Deficiency is Crucial for 'On-Time' Embryo Implantation that Directs Subsequent Development. Development 129:2879-2889

Song G., Satterfield MC., Kim J., Bazer FW., Spencer TE. (2009) Progesterone and Interferon tau regulate Leukemia Inhibitory Factor Receptor and IL6ST in the Ovine Uterus during early Pregnancy. Reproduction 137:553-565

Soto P., Natzke RP., Hansen PJ. (2003a) Actions of Tumor Necrosis Factor- $\alpha$ on oocyte Maturation and Embryonic Development in Cattle. American Jounral of Reproductive Immunology 50:380-388

Soto P., Natzke RP., Hansen PJ. (2003b) Identification of Possible Mediators of Embryonic Mortality Caused by Mastitis: Actions of Lipopolysaccharide, Prostaglandin F2alpha, and the Nitric Oxide Generator, Sodium Nitroprusside dehydrate, on Oocyte Maturation and Embryonic Development in Cattle. American Journal of Reproductive Immunology 50(3):263-272

Souza DG., Fagundes CT., Amaral FA., Cisalpino D., Sousa LP., Vieira AT., Pinho V., Nicoli JR., Vieira LQ., Fierro IM., Teixeira MM. (2007) The Required Role of Endogenously Produced Lipoxin A4 and Annexin-1 for the Production of IL-10 and Inflammatory Hyporesponsiveness in Mice. Journal of Immunology 179(12):8533-8543

Spangelo BL., MacLeod RM., Isakson PC. (1990) Production of Interleukin-6 by Anterior Pituitary Cells in Vitro. Endocrinology 126:582-586

Spencer TE and Bazer FW (1996) Ovine Interferon tau Suppresses Transcription of the Estrogen Receptor and Oxytocin Receptor Genes in the Ovine Endometrium. Endocrinology 137:1144-1147

Spencer TE. and Bazer FW. (2004a) Conceptus Signals for Establishment and Maintenance of Pregnancy. Reproductive Biology and Endocrinology 2:49

Spencer TE. and Bazer FW. (2004b) Uterine and Placental Factors Regulating Conceptus Growth in Domestic Animals. Journal of Animal Science 82(E. Suppl.):E4-E13

Spencer TE., Johnson GA., Bazer FW., Burghardt RC. (2004a) Implantation Mechanisms: Insights from the Sheep. Reproduction 128:657-668

Spencer TE., Johnson RC., Burghardt RC., Bazer FW. (2004b) Progesterone, and Placental Hormone Actions on the Uterus: Insights from Domestic Animals. Biology of Reproduction 71:2-10

Spencer TE., Johnson RC., Bazer FW., Burghardt RC. (2007) Fetal-Maternal Interactions During the Establishment of Pregnancy in Ruminants. Society of Reproduction and Fertility Supplement 64:379-396

Spencer TE., Sandra O., Wolf E. (2008) Genes Involved in Conceptus-Endometrial Interactions in Ruminants: Insights from Reductionism and Thoughts on Holistic Approaches. Reproduction 135:165-179

Spite M., Norling LV., Summers L., Yang R., Cooper D., Petasis NA., Flower RJ., Perretti M., Serhan CN. (2009) Resolvin D2 is a Potent Regulator of Leukocytes and Controls Microbial Sepsis. Nature 461(7268):1287-1291

Spolski R., Kim Hp., Zhu W., Levy DE., Leonard WJ. (2009) IL-21 Mediates Suppressive Effects via its Induction of IL-10. Journal of Immunology 182(5):2859-2867

Sporn MB. (1999) TGF- $\beta: 20$ Years and Counting. Microbes and Infection 1(15):1251-1253

Staples CR., Burke JM., Thatcher WW. (1998) Influence of Supplemental Fats on Reproductive Performance of Lactating Cows. Journal of Dairy Science 81:856-871

Steinman RM., Hawiger D., Nussenzweig MC. (2003) Tolerogenic Dendritic Cells. Annual Review of Immunology 21:685-711 
Stewart CL., Kaspar P., Brunet LJ., Bhatt H., Gadi I., Köntgen F., Abbondanzo SJ. (1992) Blastocyst Implantation depends on Maternal Expression of Leukemia Inhibitory Factor. Nature 359(6390):76-79

Stewart MD., Johnson GA., Burghardt RC., Schuler LA., Joyce MM., Bazer FW., Spencer TE. (2000) Prolactin Receptor and Uterine Milk Protein Expression in the Ovine Uterus. Biology of Reproduction 62:1779-1789

Stillman BN., Hsu DK., Pang M., Brewer CF., Johnson P., Liu F-T., Baum LG. (2006) Galectin-3 and Galectin-1 Bind Distinct Cell Surface Glycoprotein Receptors to Induce T Cell Death. Journal of Immunology 176:778-789

Stitt JT. (1986) Prostaglandin E as the Neural Mediator of the Febrile Response. Yale Journal of Biology and Medicine 59(2):137-149

Strober W., Kelsall B., Fuss I., Marth T., Ludviksson B., Ehrhardt R., Neurath M. (1997) Reciprocal IFN gamma and TGF beta Response Regulate the Occurrence of Mucosal Inflammation. Immunology Today 18(2):61-64

Stubbs CD. and Smith AD. (1984) The Modification of Mammalian Membrane Polyunsaturated Fatty Acid Composition in Relation to Membrane Fluidity and Function. Biochimica et Biophysica Acta 779:89-137

Su L., Sun Y., Ma F., Lü P., Huang H., Zhou J. (2009) Progesterone Inhibits Toll-Like Receptor 4-Mediated Innate Immune Response in Macrophages by Suppressing NF-kappa B Activation and Enhancing SOCS1 Expression. Immunology Letters 125(2):151-155

Suriyasathaporn W., Heuer C., Noordhuizen-Stassen EN., Schukken H. (2000) Hyperketonemia and the Impairment of Udder Defense: A Review. Veterinary Research 31:397-412

Sutterwala FS., Ogura Y., Szczepanik M., Lara-Tejero M., Lichtenberger GS., Grant EP., Bertin J., Coyle AJ., Galán JE., Askenase PW., Flavell RA. (2006) Critical Role for NALP3/CIAS1/Cryopyrin in Innate and Adaptive Immunity through its Regulation of Caspase-1. Immunity 24(3):317-327

Suzuki S., Toné S., Takikawa O., Kubo T., Kohno I., Minatogawa Y. (2001) Expression of indoleamine 2,3dioxygenase and Tryptophan 2,3-dioxygenase in Early Concepti. Biochemical Journal 355:425-429

Szanto A. and Nagy L. (2008) The Many faces of PPAR gamma: Anti-Inflammatory by any Means? Immunobiology 213(9-10):789-803

Szekeres-Bartho J. and Wegmann TG. (1996) A Progesterone Dependent Immunomodulatory Protein alters the Th1 /Th2 Balance. Journal of Reproductive Immunology 31(1-2):81-95

Szekeres-Bartho J., Faust Z., Varga P., Szereday L., Kelemen K. (1996) The Immunological Pregnancy Protective Effect of Progesterone is Manifested via Controlling Cytokine Production. American Journal of Immunology 35(4):348-351

Tabiasco J., Rabot M., Aguerre-Girr M., El Costa H., Berrebi A., Parant O., Laskarin G., Juretic K., Bensussan A., RukavinaD., Le Bouteiller P. (2006) Human Decidual NK Cells: Unique Phenotype and Functional Properties-A Review. Placenta 27 Suppl A: S34-S39

Tafuri A., Alferink J., Moller P., Hammerling GJ., Arnold B. (1995) T Cell Awareness of Paternal Alloantigens During Pregnancy. Science 270:630-633

Tai P., Wang J., Jin H., Song X., Yan J., Kang Y., Zhao L., An X., Du X., Chen X., Wang S., Xia G., Wang B. (2008) Induction of Regulatory T Cells by Physiological Level Estrogen. Journal of Cellular Physiology 214(2):456-464

Takahashi T., Tagami T., Yamazaki S., Uede T., Shimizu J., Sakaguchi N., Mak TW., Sakaguchi S. (2000) Immunologic Self-Tolerance Maintained by CD25+CD4+ Regulatory T Cells Constitutively Expressing Cytotoxic T Lymphocyte-Associated Antigen 4. Journal of Experimental Medicine 192(2):303-309 
Takano T., Clish CB., Gronert K., Petasis N., Serhan CN. (1998) Neutrophil-Mediated Changes in Vascular Permeability are Inhibited by Topical Application of Aspirin-Triggered 15-epi-lipoxin A4 and Novel Lipoxin B4 Stable Analogues. The Journal of Clinical Investigation 101(4):819-826

Takano H., Nagai T., Asakawa M., Toyozaki T., Oka T., Komuro I., Saito T., Masuda Y. (2000) Peroxisome Proliferator- Receptor Activators Inhibit Lipopolysaccharide- Induced Tumor Necrosis Factor-Alpha Expression in Neonatal Rat Cardiac Myocytes. Circulation Research 87(7):596-602

Takeuchi O., Hoshino K., Akira S. (2000) Cutting Edge: TLR2-Deficient and MyD88-Deficient Mice are Highly Susceptible to Straphylococcus aureus Infection. Journal of Immunology 165:5392-5396

Takeuchi O. and Akira S. (2010) Pattern Recognition Receptors and Inflammation. Cell 140(6):805-820

Tao Y., Li Y., Piao H., Zhou W., Zhang D., Fu Q., Wang S., Li D., Du M. (2015) CD56 ${ }^{\text {bright }}$ CD25+ NK Cells are preferentially Recruited to the Maternal/Fetal Interface in Early Human Pregnancy. Cellular and Molecular Immunology 12:77-86

Tawfik OW., Hunt JS., Wood GW. (1986) Implication of Prostaglandin E2 in Soluble Factor-Mediated Immune Suppression by Murine Decidual Cells. American Journal of Reproductive Immunology and Microbiology 12(4):111-117

Tekin S. and Hansen PJ. (2004) Regulation of Numbers of Macrophages in the Endometrium of the Sheep by Systemic Effects of Pregnancy, Local Presence of the Conceptus, and Progesterone. American Journal of Reproductive Immunology 51:56-62

Telleria CM., Ou J., Sugino N., Ferguson S., Gibori G. (1998) The Expression of Interleukin-6 in the Pregnant Rat Corpus Luteum and its Regulation by Progesterone and Glucocorticoid. Endocrinology 139(8):3597-3605

Terness P., Bauer TM., Röse L., Dufter C., Watzlik A., Simon H., Opelz G. (2002) Inhibition of Allogeneic T Cell Proliferation by Indoleamine 2,3-dioxygenase-Expressing Dendritic Cells: Mediation of Suppression by Tryptophan Metabolites. Journal of Experimental Medicine 196(4):447-457

Than NG., Romero R., Kim CJ., McGown MR., Papp Z., Wildman DE. (2012) Galectins: Guardians of Eutherian Pregnancy at the Maternal-Fetal Interface. Trends in Endocrinology and Metabolism 23:23-31

Thatcher WW., Bilby TR., Bartolome F., Silvelstre CR., Staples JE., Santos JE. (2006) Strategies for Improving Fertility in the Modern Dairy Cow. Theriogenology 65:30-44

Thatcher WW., Santos JEP., Silvestre FT., Kim IH., Staples CR. (2010) Perspective on Physiological/Endocrine and Nutritional Factors Influencing Fertility in Post-Partum Dairy Cows. Reproduction in Domestic Animals 45(Suppl. 3):2-14

Thatcher W., Santos JEP., Staples CR. (2011) Dietary Manipulations to Improve Embryonic Survival in Cattle. Theriogenology 76: 1619-1631

Thomas DA. and Massaqué J. (2005) TGF-beta Directly Targets Cytotoxic T Cell Functions during Tumor Evasion of Immune Surveillance. Cancer Cell 8(5):369-380

Thornton AM. and Shevach EM. (1998) CD4+CD25+ Immunoregulatory T Cells Suppress Polyclonal T Cell Activation in Vitro by Inhibiting Interleukin 2 Production. Journal of Experimental Medicine 188(2):287-296

Thornton AM. And Shevach EM (2000) Suppressor Effector Function of CD4 +CD25+ Immunoregulatory T Cells is Antigen Nonspecific. Journal of Immunology 164:183-190Guerin

Tirado-González I., Freitag N., Barrientos G., Shaikly V., Nagaeva O., Strand M., Kjellberg L., Klapp BF., Mincheva-Nilsson L., Cohen M., Blois SM. (2013) Galectin-1 Influences Trophoblast Immune Evasion and Emerges as a Predictive Factor for the Outcome of Pregnancy. Molecular Human Reproduction 19:43-53 
Toscano MA., Bianco GA., Ilarregui JM., Croci DO., Correale J., Hernandez JD., Zwirner NW., Poirier F., Riley EM., Baum LG., Rabinovich GA. (2007) Differential Glycosylation of TH1, TH2, and TH17 Effector Cells Selectively Regulates Susceptibility to Cell Death. Nature Immunology 8(8):825-834

Toscano MA., Campagna L., Molinera LL., Cerliani JP., Croci DO., Ilarregui JM., Fuerter MB., Nojek IM., Fededa JP., Zwirner NW., Costas MA., Rabinovich GA. (2011) Nuclear Factor(NF)-кB Controls Expression of the Immunoregulatory Glycan-Binding Protein Galectin-1. Molecular Immunology 48:1940-1949

Tracey KJ. (2002) The Inflammatory Reflex. Nature 420:853-859

Tremellen KP., Seamark RF., Robertson SA. (1998) Seminal Transforming Growth Factor beta 1 Stimulates Granulocyte-Macrophage Colony-Stimulating Factor Production and Inflammatory Cell Recruitment in the Murine Uterus. Biology of Reproduction 58(5):1217-1225

Trowsdale J and Betz AG. (2006) Mother's Little Helpers: mechanisms of Maternal-Fetal Tolerance. Nature Immunology 7(3):241-246

Turnbull AV. And Rivier CL. (1999) Regulation of the Hypothalamic-Pituitary-Adrenal Axis by Cytokines: Actions and Mechanisms of Action. Physiological Reviews 79:1-71

Turner ML., Healey GD., Sheldon IM. (2012) Immunity and Inflammation in the Uterus. Reproduction in Domestic Animals 47:402-409

Uribe HA., Kennedy BW., Martin SW., Kelton DF. (1995) Genetic Parameters for Common health Disorders of Holstein Cows. Journal of Dairy Science 78:421-430

Ushikubi F., Segi E., Sugimoto Y., Murata T., Matsuoka T., Kobayashi T., Hizaki H., Tuboi K., Katsuyama M., Ichikawa A., Tanaka T., Yoshida N., Narumiya S. (1998) Impaired Febrile Response in Mice Lacking the Prostaglandin E Receptor Subtype EP3. Nature 395(6699):281-284

Vacca P., Cantoni C., Vitale M., Prato C., Canegallo F., Fenoglio D., Ragni N., Moretta L., Mingari MC. (2010) Crosstalk Between Decidual NK and CD14+ Myleomonocytic Cells Results in Induction of Tregs and Immunosuppression. Proceedings of the National Academy of Sciences of the U.S.A. 107:11918-11923

Vacca P., Moretta L., Moretta A., Mingari MC. (2011a) Origin, Phenotype and Function of Human Natural Killer Cells in Pregnancy. Trends in Immunology 32:517-523

Vacca P., Vitale C., Montaldo E., Conte R., Cantoni C., Fulcheri E., Darretta V., Moretta L., Mingari MC. (2011b) CD34+ Hematopoietic Precursors are Present in Human Decidua and Differentiate into Natural Killer Cells upon Interaction with Stromal Cells. Proceedings of the National Academy of Sciences of the United States of America 108(6):2402-2407

van der Pouw Kraan TC., Boeije LC., Smeenk RJ., Wijdenes J., Aarden LA. (1995) Prostaglandin-E2 is a Potent Inhibitor of Human Interleukin 12 Production. Journal of Experimental Medicine 181(2):775-779

van Deventer SJ., Büller HR., ten Cate JW., Aarden LA., Hack CE., Sturk A. (1990) Experimental Endotoxemia in Humans: Analysis of Cytokine Release and coagulation, Fibrinolytic, and Complement Pathways. Blood 76(12):2520-2526

Van Elssen CH., Vanderlocht J., Oth T., Senden-Gijsbers BL., Germeraad WT., Bos GM. (2011) Inflammation Restraining Effects of Prostaglandin E2 on NK-DC Interaction are Imprinted during Dendritic Cell Maturation. Blood 118(9):2473-2482

Vespa GN., Lewis LA., Kozak KR., Moran M., Nhuyan JT., Baum LG., Miceli MC. (1999) Galectin-1 Specifically Modulates TCR Signals to Enhance TCR Apoptosis but Inhibit IL-2 Production and Proliferation. Journal of Immunology 162(2):799-806 
Vijay-Kumar, M., Vanegas, S.M., Patel, N., Aitken, J.D., Zieglar, T.R., Ganji, V. (2011) Fish Oil Rich Diet in Comparison to Saturated Fat Rich Diet Offered Protection Against Lipopolysaccharide and Insulin Resistance in Mice. Nutrition \& Metabolism 8:16

Vodovotz Y., Bogdan C., Paik J., Xie QW., Nathan C. (1993) Mechanisms of Suppression of Macrophage Nitric Oxide Release by Transforming Growth Factor beta. Journal of Experimental Medicine 178(2):605-613

Voll RE., Herrmann M., Roth EA., Stach C., Kalden JR., Girkontaite I. (1997) Immunosuppressive Effect of Apoptotic Cells. Nature 390:350-351

von Moltke J., Trinidad NJ., Moayeri M., Kintzer AF., Wang SB., van Rooijen N., Brown CR., Krantz BA., Leppla SH., Gronert K., Vance RE. (2012) Rapid Induction of Inflammatory Lipid Mediators by the Inflammasome in vivo. Nature 490(7418):107-111

von Wolff M., Wang X., Gabius HJ., Strowitzki T. (2005) Galectin Fingerprinting in Human Endometrium and Decidua during the Menstrual Cycle and in Early Gestation. Molecular Human Reproduction 11(3):189-194

Wachira AM., Sinclair LA., Wilkinson RG., Hallett K., Enser M., Wood JD. (2000) Rumen Biohydrogenation of n3 Polyunsaturated Fatty Acids and their Effects on Microbial Efficiency and Nutrient Digestibility in Sheep. Journal of Agricultural Science 135:419-428

Wada M., DeLong CJ., Hong YH., Rieke CJ., Song I., Sighu RS., Yuan C., Warnock M., Schmaier AH., Yokoyama C., Smyth EM., Wilson SJ., FitzGerald GA., Garavito RM., Sui de X., Regan JW., Smith WL. (2007) Enzymes and Receptors of Prostaglandin Pathways with Arachidonic Acid-Derived Versus Eicosapentaenoic Acid-Derived Substrates and Products. The Journal of Biological Chemistry 282(31):22254-22266

Wafula PO., Teles A., Schumacher A., Pohl K., Yagita H., Volk HD., Zeclussen AC. (2009) PD-1 but not CTLA-4 Blockage Abrogates the Protective Effect of Regulatory T Cells in a Pregnancy Murine Model. American Journal of Reproductive Immunology 62(5):283-292

Wahl SM., Swicher J., McCartney-Francis N., Chen W. (2004) TGF-beta: The Perpetrator of Immune Suppression by Regulatory T Cells and Suicidal T Cells. Journal of Leukocyte Biology 76:15-24

Wales RG. and Cuneo CL. (1989) Morphology and Chemical Analysis of the Sheep Conceptus from the $13^{\text {th }}$ to the $19^{\text {th }}$ Day of Pregnancy. Reproduction, Fertility, and Development 1:31-39

Walker C., Kristensen F., Bettens F., deWeck AL. (1983) Lymphokine Regulation of Activated (G1) Lymphocytes, I: Prostaglandin E2-Induced Inhibition of Interleukin 2 Production. Journal of Immunology 130(4):1770-1773

Walker W. and Rotondo D. (2004) Prostaglandin E2 is a Potent Regulator of Interleukin-12- and Interleukin-18Induced Natural Killer Cell Interferon-gamma Synthesis. Immunology 111(3): 298-305

Walker CG., Meier S., Littlejohn MD., Lehnert K., Roche JR., Mitchell MD. (2010) Modulation of the Maternal Immune System by the Pre-Implantation Embryo. BMC Genomics 11:474

Walker J., Dichter E., Lacorte G., Kerner D., Spur B., Rodriguez A., Yin K. (2011) Lipoxin A4 Increases Survival by Decreasing Systemic Inflammation and Bacterial Load in Sepsis. Shock 36(4):410-416

Walker LSK., Chodos A., Eggena M., Dooms H., Abbas AK. (2003) Antigen-Dependent Proliferation of CD4+CD25+ Regulatory T Cells in Vivo. Journal of Experimental Medicine 198(2):249

Walsh SW., Williams EJ., Evans ACO. (2011) A Review of the Causes of poor Fertility in High Milk Producing Dairy Cows. Animal Reproduction Science 123:127-138

Wan YY. And Flavell RA. (2006) The Roles for Cytokines in the Generation and Maintenance of Regulatory T Cells. Immunological Reviews 212:114-130 
Wang B., Gong X., Wan J., Zhang L., Zhang Z., Li H., Min S. (2011) Resolvin D1 Protects Mice from LPS-Induced Acute Lung Injury. Pulmonary Pharmacology \& Therapeutics 24(4):434-441

Wang H., Ma W., Tegada L., Zhang H., Morrow JD., Das SK., Dey SK. (2004) Rescue of female Infertility from the Loss of Cyclooxygenase-2 by Compensatory Up-Regulation of Cyclooxygenase-1 is a Function of Genetic Makeup. The Journal of Biological Chemistry 279(11):10649-10658

Wang H., Meng R., Li Z., Yang B., Liu Y., Huang F., Zhang J., Chen H., Wu C. (2011) IL-27 Induces the Differentiation of Tr1-like Cells from Human naive CD4+ T Cells via the Phosphorylation of STAT1 and STAT3. Immunology Letters 136:21-28

Wang P., Anderson PO., Chen S., Paulsson KM., Sjögren H-O., Li S. (2001) Inhibition of the Transcription Factors AP-1 and NF- $\mathrm{KB}$ in CD4 T Cells by Peroxisome Proliferator- Activated Receptor $\gamma$ Ligands. International Immunopharmacology 1(4):803-812

Wang W., Nan X., Ji P., Dow KE. (2007) Corticotropin Releasing Hormone Modulates Endotoxin-Induced Inflammatory Cytokine Expression in Human Trophoblast Cells. Placenta 28(10):1032-1038

Wang ZY., Sato H., Kusam S., Sehra S., Toney LM., Dent AL. (2005) Regulation of IL-10 Gene Expression in Th2 Cells by Jun Proteins. Journal of Immunology 174(4):2098-2105

Warning JC., McCracken SA., Morris JM. (2011) A Balancing Act: Mechanisms by which the Fetus avoids Rejection by the Maternal Immune System. Reproduction 141:715-724

Wathes DC., Cheng Z., Chowdhury W., Fenwick MA., Fitzpatrick R., Morris DG., Patton J., Murphy JJ. (2009) Negative Energy Balance Alters Global Gene Expression and immune Responses in the Uterus of Postpartum Dairy Cows. Physiological Genomics 39:1-13

Webster EL., Torpy DJ., Elenkov IJ., Chrousos GP. (1998) Corticotropin-Releasing Hormone and Inflammation. Annals of the New York Academy of Sciences 840:21-32

Wegmann TG., Lin H., Guilbert L., Mosmann TR. (1993) Bidirectional Cytokine Interactions in the Maternal-Fetal Relationship: is Successful Pregnancy a $\mathrm{T}_{\mathrm{H}} 2$ Phenomenon? Immunology Today 14(7):353-356

Wegorzewska M., Nijagal A., Wong CM., Le T., Lescano N., Tang Q., MacKenzie TC. (2014) Fetal Intervention Increases Maternal T Cell Awareness of the Foreign Conceptus and Can Lead to Immune-Mediated Fetal Demise. Journal of Immunology 192:1938-1945

Weylandt K-H. (2016) Docosapentaenoic Acid Derived Metabolites and Mediators-The New World of Lipid Mediator Medicine in a Nutshell. European Journal of Pharmacology 785:108-115

Wieser F., Waite L., Depoix C., Taylor RN. (2008) PPAR Action in Human Placental Development and Pregnancy and its Complications. PPAR Research 1-14

Wilhelms DB., Kirilov M., Mirrasekhian E., Eskillson A., Kugelberg UÖ., Klar C., Ridder DA., Herschman HR., Schwaninger M., Blomqvist A., Engblom D. (2014) Deletion of Prostagladin E2 Synthesizing Enzymes in Brain Endothelial Cells Attenuates Inflammatory Fever. Journal of Neuroscience 34(35):11684-11690

Willems F., Marchant A., Delville JP., Gérard C., Delvaux A., Velu T., de Boer M., Goldman M. (1994) Interleukin10 Inhibits B7 and Intercellular Adhesion Molecule-1 Expression on Human Monocytes. European Journal of Immunology 24(40:1007-1009

Williams TJ. and Peck MJ (1977) Role of Prostaglandins-Mediated Vasodilation in Inflammation. Natuer 270:530532

Williams MR., Azcutia V., Newton G., Alcaide P., Luscinskas FW. (2011) Emerging Mechanisms of Neutrophil Recruitment across Endothelium. Trends in Immunology 32(10):461-469 
Wiltbank MC., Baez GM., Garcia-Guerra A., Toledo MZ., Monteiro PLJ., Melo LF., Ochoa JC., Santos JEP., Sartori R. Pivotal Periods for Pregnancy Loss during the First Trimester of Gestation in Lactation Dairy Cows. Theriogenology 86:239-253

Wimsatt WA. (1950) New Histological Observations on the Placenta of the Sheep. American Journal of Anatomy $87: 391-436$

Winger EE., Reed JL. (2013) The Multiple Faces of the Decidual Natural Killer Cell. American Journal of Reproductive Immunology 70:1-9

Wira CR and Rossoll RM. (1995) Antigen-Presenting Cells n the Female Reproductive Tract: Influence of the estrous Cycle on Antigen Presentation by Uterine Epithelial and Stromal Cells. Endocrinology 136(10):4526-4534

Wira CR., Grant-Tschudy KS., Crane-Godreau MA. (2005) Epithelial Cells in the Female Reproductive Tract: a Central Role as Sentinels of Immune Protection. American Journal of Reproductive Immunology 53:65-76

Wise MJ., (2016) Potential Biomarkers to Predict Fertility in Dairy Cattle. Thesis West Virginia University ProQuest Number: 10110069

Woidacki K., Meyer N., Schumacher A., Goldschmidit A., Mauer M., Zenclussen AC. (2015) Transfer of Regulatory T Cells into Abortion-Prone Mice Promotes the Expansion of uterine Mast Cells and Normalized Early Pregnancy Angiogenesis. Scientific Reports 5:13938

Wooding FB., Morgan G., Forsyth IA., Butcher G., Hutchings A., Billingsley SA., Gluckman PD. (1992) Light and Electron Microscope Studies of Cellular Localization of oPL with Monoclonal and Polyclonal Antibodies. Journal of Histochemistry Cytochemistry 40: 1001-1009

Wonsil BJ., Herbein JH., Watkins BA. (1994) Dietary and Ruminally Derived trans-18:1 fatty Acids Alter Bovine Milk Lipids. The Journal of Nutrition 124(4): 556-565

Wu CY., Wang K., McDyer JF., Seder RA. (1998) Prostaglandin E2 and Dexamethasone Inhibit IL-12 Receptor Expression and IL-12 Responsiveness. Jounral of Immunology 161(6):2723-2730

Wuu Y-D., Pampfer S., Becquet P., Vanderheyden I., Lee K-H., Hertogh RD. (1999) Tumor Necrosis Factor $\alpha$ Decreases the Viability of Mouse Blastocysts in Vitro and in Vivo. Biology of Reproduction 60:479-483

Xu D., Chan WL., Leung BP., Hunter D., Schulz K., Carter RW., Mclnnes IB., Robinson JH., Liew FY. (1998) Selective Expression and Functions of Interleukin 18 Receptor on T Helper (Th) Type 1 but not Th2 Cells. Journal of Experimental Medicine 188(8):1485-1492

Xu C., Mao D., Holers VM., Palanca B., Cheng AM., Molina H. (2000) A Critical Role for Murine Complement Regulator Crry in Fetomaternal Tolerance. Science 287:498-501

Xu A., Zhang L., Liu T., Park JY., Berta T., Yang R., Serhan CN., Ji R-R. (2010) Resolvins RvE1 and RvD1 Attenuate Inflammatory Pain via Central and Peripheral Actions. Nature Medicine 16:592-597

Yamamoto M., Sato S., Hemmi H., Hoshino K., Kaisho T., Sanjo H., Takeuchi O., Sugiyama M., Okabe M., Takeda K., Akira S. (2003) Role of Adaptor TRIF in the MyD88-Independent Toll-Like Receptor Signaling Pathway. Science 301:640-643

Yamaoka K., Otsuka T., Nirro H., Arinobu Y., Niho Y., Hamasaki N., Izuhara K. (1998) Activation of STAT5 by Lipopolysaccharide through Granulocyte-Macroophage Colony-Stimulating Factor Production in Human Monocytes. Journal of Immunology 160(2):838-845

Yamaoka K., Otsuka T., Niiro H., Nakashima H., Tanaka Y., Nagano S., Ogami E., Niho Y., Hamasaki N., Izuhara K. (1999) Selective DNA-Binding Activity of Interleukin-10 Stimulated STAT Molecules in Human Monocytes. Journal of Interferon and Cytokine Research 19(6):679-685 
Yie SM., Xiao R., Librach CL. (2006) Progesterone Regulates HLA-G Gene Expression through a Novel Progesterone Response Element. Human Reproduction 21(10):2538-2544

Yoshimoto T., Takeda K., Tanaka T., Ohkusu K., Kashiwamura S., Okamura H., Akira S., Nakanishi K. (1998) IL12 Up-Regulates IL-18 Receptor Expression on T Cells, Th1 Cells, and B Cells: Synergism with IL-18 for IFN $\gamma$ Production. Journal of Immunology 161(7):3400-3407

Yoshinaga K. (1988) Uterine Receptivity for Blastocyst Implantation. Annals of the New York Academy of Sciences $541: 424-431$

Yoshinaga K. (2012) Two Concepts on the Immunological Aspect of Blastocyst Implantation. Journal of Reproduction and Development 58(2):196-203

Yui J., Garcia-Lloret M., Wegmann TG., Guilbert LJ. (1994) Cytotoxicity of Tumor Necrosis Factor-Alpha and Gamma-Interferon against Primary Human Placental Trophoblasts. Placenta 15(8):819-835

Zanclussen AC., Blois S., Stumpo R., Olmos S., Arias K., Malan Borel I., Margni RA. (2003) Murine Abortion is Associated with Enhanced interleukin-6 Levels at the Feto-Maternal Interface. Cytokine 24(4):150-160

Zarrin M., Wellnitz O., van Dorland HA., Bruckmaier RM. (2014) Induced Hyperketonemia Affects the Mammary Immune Response during Lipopolysaccharide Challenge in Dairy Cows. Journal of Dairy Science 97:330-339

Zenclussen AC., Gerlof K., Zenclussen ML., Sollwedel A., Bertoja AZ., Ritter T., Kotsch K., Leber J., Volk H-d. (2005) Abnormal T-Cell Reactivity against Paternal Antigens in Spontaneous Abortion: Adoptive Transfer of Pregnancy-Induced CD4+CD25+ T Regulatory Cells Prevents Fetal Rejection in a Murine Abortion Model. American Journal of Pathology 166(3):811-822

Zhang S., Lin H., Kong S., Wang S., Wang H., Wang H., Armant DR. (2013) Physiological and Molecular Determinants of Embryo Implantation. Molecular Aspects of Medicine 34(5):939-980

Zhang Y-H., Tian M., Tang M-X., Liu Z-Z., Liao A-H. (2015) Recent Insight into the Role of the PD-1/PD-L1 Pathway in Feto-Maternal Tolerance and Pregnancy. American Journal of Reproductive Immunology 74:201-208

Zhao JX., Zeng YY., Liu Y. (2007) Fetal Alloantigen is Responsible for the Expansion of the CD4(+)CD25(+) Regulatory T cell Pool during Pregnancy. Journal of Reproductive Immunology 75(2):71-81

Zheng SG., Wang J., Horwitz DA. (2008) Cutting Edge: Foxp3+CD4+CD25+ Regulatory T Cells Induced by IL-2 and TGF- are Resistant to Th17 Conversion by IL-6. Journal of Immunology 180(199):7112-7116

Zheng Y., Josefowicz S., Chaudhry A., Peng XP., Forbush K., Rudensky AY. (2010) Role of Conserved Non-

Coding DNA Elements in the Foxp3 Gene in Regulatory T-cell Fate. Nature 463(7282):808-812

Zhou L., Chong MM., Littman DR. (2009) Plasticity of CD4+ T Cell Lineage Differentiation. Immunity 30(5):646655

Zhu XY., Zhou YH., Wang MY., Jin LP., Yuan MM., Li DJ. (2005) Blockade of CD86 Signaling Facilitates a Th2 Bias at the Maternal-Fetal Interface and Expands Peripheral CD4+CD25+ Regulatory T Cells to Rescue AbortionProne Fetuses. Biology of Reproduction 72(2):338-345 
